\title{
MIXING AND DYNAMICS OF THE MEDITERRANEAN OUTFLOW
}

by

Molly O'Neil Baringer

B.S. Newcomb College of Tulane University

Submitted in partial fulfillment of the requirements for the degree of

Doctor of Philosophy

\begin{tabular}{c} 
MAAINE \\
BIOLOGICAL \\
LABORATORY \\
\hline LIBRARY \\
\hline $\begin{array}{c}\text { WODS HOLE, MASS. } \\
\text { W. H. O.1. }\end{array}$ \\
\hline
\end{tabular}

at the

\section{MASSACHUSETTS INSTITUTE OF TECHNOLOGY}

and the

\section{WOODS HOLE OCEANOGRAPHIC INSTITUTION}

October 1993

(C) Molly O. Baringer 1993

The author hereby grants to MIT and to WHOI permission to reproduce and to distribute copies of this thesis document in whole or in part.

Signature of Author .... . . .

Joint Program in Physical Oceanography Massachusetts Institute of Technology Woods Hole Oceanographic Institution October 1, 1993

Certified by

James Price

Associate Scientist in Physical Oceanography

Thesis Supervisor

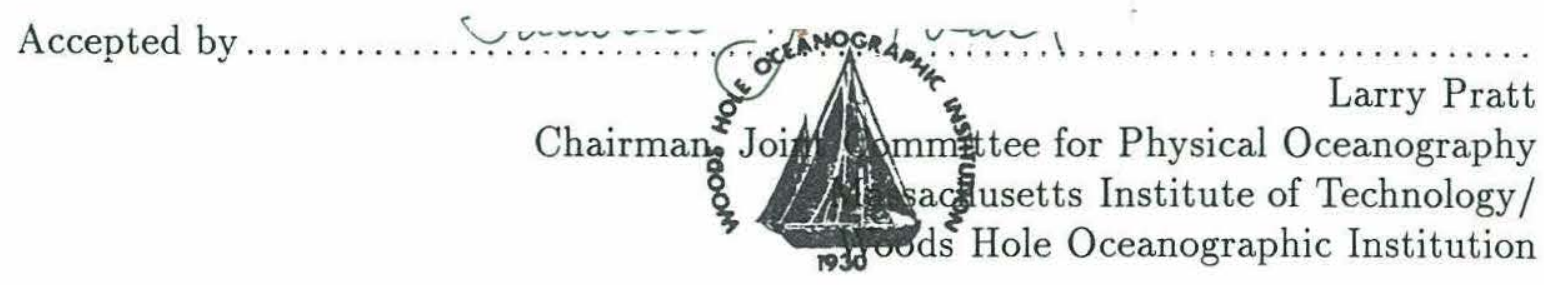




\title{
MIXING AND DYNAMICS OF THE MEDITERRANEAN OUTFLOW by \\ Molly O'Neil Baringer
}

\author{
Submitted in partial fulfillment of the requirements for the degree of \\ Master of Science at the Massachusetts Institute of Technology \\ and the Woods Hole Oceanographic Institution \\ October 1, 1993
}

\begin{abstract}
Hydrographic and expendable current profiler (XCP) data taken during the Gulf of Cadiz Expedition in September 1988 are analyzed to diagnose the mixing and dynamics of the Mediterranean outflow. The overall structure of the outflow is consistent with that described in the historical literature (Heezen and Johnson, 1969). This data shows that the overflow transport doubles from $.85 \mathrm{~Sv}$ to $1.9 \mathrm{~Sv}$, and that the velocity weighted salinity decreases from 37.8 pss to $36.7 \mathrm{pss}$ in the first $60 \mathrm{~km}$ of the path. The core salinity of the neutrally buoyant outflow near Cape St. Vincent is 36.6 pss, which indicates that most of the mixing has taken place close to the Strait in the initial descent of the outflow.

Cross stream variations in the overflow $\mathrm{T} / \mathrm{S}$ properties increase as the flow spreads from $10 \mathrm{~km}$ to $90 \mathrm{~km}$ wide. The outflow begins with less than a $0.5^{\circ} \mathrm{C}$ acrossstream variation in temperature in the Strait with the saltiest, coldest water to the south and slightly fresher and warmer outflow to the north. As the outflow spreads, the northern near-shelf flow follows a path higher in the water column and mixes with warmer North Atlantic water than does the deeper offshore flow. Within the first $100 \mathrm{~km}$, the cross stream variation in temperature on an isopycnal becomes more than a $2^{\circ} \mathrm{C}$. The flow eventually settles along two preferred isopycnals: 27.5 and 27.8 (Zenk 1975b). The spreading of the flow contains both a barotropic and baroclinic character. The average change in angle above and below the maximum velocity of the outflow is $8^{\circ}$ while at the edges of the flow the average direction of the outflow diverges by as much as $50^{\circ}$.

Gradient Richardson numbers less than $1 / 4$ are found in the interface (up to 50 $\mathrm{m}$ thick) between westward flowing Mediterranean water and eastward flowing North Atlantic water, even though there is a strong stabilizing stratification present. Bulk Froude numbers greater than 1 are found near the Strait coincident with the vigorous mixing noted above. Lower bulk Froude numbers were observed in regions where less entrainment was taking place.
\end{abstract}


The momentum balances are diagnosed using hydrographic and XCP data. Evaluation of the cross stream momentum balance shows the importance of advection as the flow makes a 90 degree inertial turn upon entering the Gulf of Cadiz. A form of the Bernoulli function can be evaluated to infer the total stress (entrainment and bottom drag) acting on the outflow. This stress is as large as $5 \mathrm{~Pa}$ within $20 \mathrm{~km}$ of the Strait, while further downstream the stress decreases to about $\frac{1}{2} \mathrm{~Pa}$. The entrainment stress estimated from the property fluxes reaches a maximum of about $0.8 \mathrm{~Pa}$ near section $\mathrm{C}$, indicating that bottom stress is dominant. Near the Strait, advection, bottom drag and the Coriolis force are all critical to the dynamics of the outflow. Further downstream, the outflow becomes a damped geostrophic current. A simple geostrophic adjustment model is used to show that in the absence of frictional stresses, the outflow would very quickly become geostrophically balanced and descend only about $10 \mathrm{~m}$ down the continental slope. Thus, friction is critical for the outflow to cross isobaths.

A simple numerical model that uses a Froude number dependent entrainment and a quadratic bottom friction law is used to simulate the outflow (Price and Baringer, 1993). Some of the properties of the outflow including localized entrainment, large stresses and high Rossby number of the flow (initially as high as 0.6 ), are simulated rather well, though the model overestimates the magnitude of the outflow current. We suspect that this is a consequence of assuming a passive ocean.

Two different methods for specifying the broadening of the flow are compared: one using the highly parameterized concept of Ekman spreading, the other using the conservation of potential vorticity. The potential vorticity broadening more accurately reproduces the observed width of the flow near Cape St. Vincent where the width varies inversely with the bottom slope. However, both methods produce essentially the same equilibrium temperature, salinity and transport of the outflow which is a testament to the robustness of the model solution. with the formation process of NADW.

Thesis Supervisor: James Price, Associate Scientist in Physical Oceanography Woods Hole Oceanographic Institution 


\section{Contents}

1 Introduction 16

1.1 The Mediterranean Sea and Its Influence on the North Atlantic . . . 17

1.2 Observations of the Mediterranean Outflow in the Strait of Gibraltar 24

1.3 Observations of the Mediterranean Outflow in the Gulf of Cadiz . . . 28

1.4 Models of the Mediterranean Outflow and other Density Currents . . 32

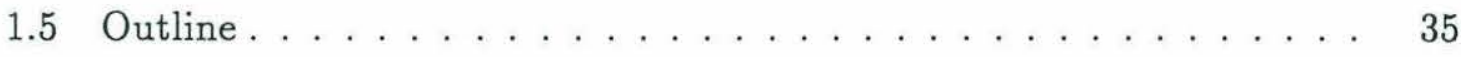

2 Gulf of Cadiz Expedition $\quad 37$

2.1 The Gulf of Cadiz Field Program . . . . . . . . . . . . . . . 38

2.2 Hydrographic and XCP Sections . . . . . . . . . . . . . 40

2.3 Determination of the absolute velocity . . . . . . . . . 71

2.4 Horizontal structure . . . . . . . . . . . . . . . . . . 73

2.5 Entrainment . . . . . . . . . . . . . . . . . . 92

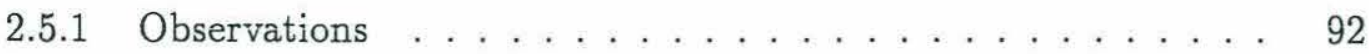


2.5.2 Richardson Numbers . . . . . . . . . . . . . . . . . . . 103

2.6 Dynamics . . . . . . . . . . . . . . . . . . . . 115

2.6.1 Integrated Momentum and Continuity Equations . . . . . . 115

2.6.2 Cross-stream momentum . . . . . . . . . . . . . . . . . . 118

2.6.3 Along-stream Momentum Equation . . . . . . . . . . . . . 122

2.6.4 Continuity and Density Equation . . . . . . . . . . . 131

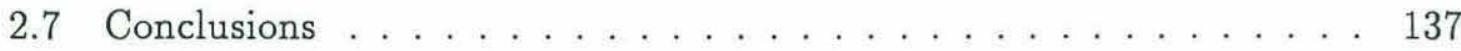

3 A Simulation of the Mediterranean Outflow 140

3.1 Model Formulation . . . . . . . . . . . . . . . . . . . . . . . . 141

3.1 .1 Implementation . . . . . . . . . . . . . . . . . . . 149

3.1 .2 Numerical stability . . . . . . . . . . . . . . . . . . 150

3.2 Developing intuition: simple analytical solutions . . . . . . . . 153

3.3 Mediterranean outflow simulation and comparison with the $1988 \mathrm{Cadiz}$ Expedition data . . . . . . . . . . . . . . . . . 159

3.3.1 Overview . . . . . . . . . . . . . . . . . . 159

3.3.2 Property Evolution . . . . . . . . . . . . . . . . . . 161

3.3.3 Dynamical Balances in the Model . . . . . . . . . . . . 166

3.4 Model Sensitivity . . . . . . . . . . . . . . . . . . . . . . . 172

3.4.1 Changes in Initial Temperature and Salinity . . . . . . . 172 
3.4.2 Changes in the Initial Height $\ldots \ldots \ldots \ldots \ldots$

3.4.3 Changes in the Initial Speed . . . . . . . . . . . . 175

3.4.4 Changes in the Bottom Drag Coefficient . . . . . . 177

3.4 .5 Summary . . . . . . . . . . . . . . . . . . . 179

3.5 Conserving Potential Vorticity to Obtain a Constraint on the Width . 182

$3.5 .1 \quad$ Basic Equations . . . . . . . . . . . . . . . . . . 182

3.5.2 Possible Boundary Conditions . . . . . . . . . . . . 184

3.5 .3 Application . . . . . . . . . . . . . . . . . 185

3.5 .4 Implementation . . . . . . . . . . . . . . . 188

3.5 .5 Results . . . . . . . . . . . . . . . . . . . . . . . . 189

3.6 Summary and Conclusions . . . . . . . . . . . . . . . . . . . . . 194

4 Summary and Discussion $\quad 197$

4.1 What does the data tell us? . . . . . . . . . . . . . . . . 198

4.2 What does the model tell us? . . . . . . . . . . . . . . . 200

4.3 Discussions and Speculations . . . . . . . . . . . . . . . . . . 202

$\begin{array}{ll}\text { A Determining the Absolute Velocity } & 207\end{array}$

A.1 Determining the top of the Mediterranean Outflow . . . . . . . 208

A.2 Why use a reference velocity at the temperature minimum? . . . . 210

A.3 Conserving salt flux . . . . . . . . . . . . . . . . . 211 
A.4 Justification for conserving salt flux . . . . . . . . . . . . . . . . . 212

A.4.1 Results...................... 215

A.5 Sensitivity to reference level . . . . . . . . . . . . . . . 216

A.6 Comparison with historical salinity minimum reference level . . . . . 217

A.7 Summary and comments . . . . . . . . . . . . . . . 218

B What is the residual of the downstream momentum budget? 220

C Layered Pressure Gradient $\quad 224$

$\begin{array}{ll}\text { References } & 231\end{array}$ 


\section{List of Figures}

1.1 Total Heat Flux into the Mediterranean . . . . . . . . . . . . . 19

1.2 Wust's Schematic of the Circulation in Mediterranean Sea . . . . . 21

1.3 Reid's (1979) salinity on an isopycnal in the North Atlantic . . . . . 23

1.4 Time series of along Strait of Gibraltar velocity from Bryden, Candela and Kinder, $1993 \ldots \ldots \ldots \ldots \ldots \ldots \ldots$

1.5 Hydraulic Schematic from Armi and Farmer, $1988 \ldots \ldots \ldots . . \ldots 27$

1.6 Madelain's (1970) outflow pathways . . . . . . . . . . . . . . 29

1.7 Cadiz Bathymetry . . . . . . . . . . . . . . . . . . 30

2.1 Station plan of CTD section locations . . . . . . . . . . . 39

2.2 Station plan of XCP section locations . . . . . . . . . . . 40

2.3 Salinity Sections . . . . . . . . . . . . . . . . . . . . . 41

2.4 Temperature Sections . . . . . . . . . . . . . . . . . . . . 46

2.5 Potential Density Sections _ . . . . . . . . . . . . . 51

2.6 Core Salinity Section . . . . . . . . . . . . . . . 57 
2.7 Maximum Salinity Map . . . . . . . . . . . . . . . . 60

2.8 XCP Velocity Sections $\ldots \ldots \ldots \ldots \ldots$

2.9 Geostrophic Velocity Sections . . . . . . . . . . . . . . . 66

2.10 Salinity section near $8^{\circ} 30^{\prime} \mathrm{W} \ldots \ldots \ldots \ldots \ldots$

2.11 Velocity Weighted Salinity Map . . . . . . . . . . . . . . 79

2.12 Minimum Salinity Map . . . . . . . . . . . . . . . . . . . . 82

2.13 Velocity Weighted $\mathrm{T}, \mathrm{S}$ plots $\ldots \ldots \ldots \ldots . \ldots . \ldots . \ldots 3$

2.14 Maximum XCP velocity of the outflow and XCP velocity at the salinity

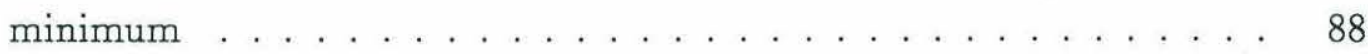

$2.15 \mathrm{XCP}$ velocity, $\mathrm{S}$ and $\mathrm{Ri}$ for $\mathrm{CTD} 65,76,83$ and $86 \ldots \ldots 89$

2.16 Velocity map 10 and $40 \mathrm{db}$ above the bottom . . . . . . . . 90

2.17 Salinity versus downstream distance . . . . . . . . . . . 95

2.18 Outflow transport versus downstream distance . . . . . . . . . 96

2.19 Section transport in $\sigma_{\theta}$ and depth classes $\ldots \ldots \ldots \ldots \ldots 10 \ldots$

2.20 Schematic of Kelvin-Helmholtz billows . . . . . . . . . . . 106

2.21 Repeat occupations of station $75 \ldots \ldots \ldots \ldots \ldots$

2.22 Bulk Froude Number at section C . . . . . . . . . . . . . . 111

2.23 Bulk Froude Number Map . . . . . . . . . . . . . . . . . 113

2.24 Schematic of idealized Potential Vorticity conserving outflow . . . . 121

2.25 Along Stream Momentum Balance . . . . . . . . . . . . . . 128 
2.26 Entrainment Rate . . . . . . . . . . . . . . . . . . . 132

3.1 Model geometry and density profile . . . . . . . . . . . . . . 141

3.2 Entrainment and Bottom Stress vs. Froude number . . . . . . . . 145

3.3 Schematic of Spreading $\ldots \ldots \ldots \ldots \ldots \ldots$

3.4 The effect of friction in determining the period of a cycloid motion . 156

3.5 Speed of a cycloid trajectory modified by friction . . . . . . 156

3.6 Path of the simulated Mediterranean outflow in the Gulf of Cadiz . . 160

3.7 Simulated outflow $\mathrm{T} / \mathrm{S}$, speed and density $\ldots \ldots \ldots \ldots 16 \ldots$

3.8 Downstream evolution of simulated outflow temperature, salinity and density . . . . . . . . . . . . . . . . . 162

3.9 Simulated outflow $h, W$, depth, area $\ldots \ldots \ldots \ldots \ldots$

3.10 Simulated outflow $F r_{b}$, Source water fraction, transport, and bottom slope . . . . . . . . . . . . . . . . . . . 164

3.11 Simulated outflow entrainment rate . . . . . . . . . . . . 165

3.12 Simulated outflow momentum balance . . . . . . . . . . 167

3.13 Simulated outflow energy balance . . . . . . . . . . . . . 171

3.14 Model Sensitivity to Different Initial Salinities . . . . . . . . . . 174

3.15 Model Sensitivity to varying initial height, temperature, speed and bottom drag coefficient . . . . . . . . . . 176

3.16 Model Solution for $c_{d}=0.001 \ldots \ldots \ldots \ldots \ldots$ 
3.17 Schematic of Uniform Potential Vorticity Layer . . . . . . . . . 183

3.18 Width Solution with Stagnant Region Boundary Conditions . . . . 186

3.19 Width Solution with Vanishing Layer Thickness Boundary Conditions 188

3.20 Model Solution with Variable Potential Vorticity . . . . . . . . 190

A.1 CTD 97 and CTD $98 \ldots \ldots \ldots \ldots \ldots \ldots$

A.2 Geostrophic Velocity Section VI from Ochoa and Bray (1991) _. . . 213

A.3 Section D Transport Versus $\sigma_{\theta}$ Reference Level . . . . . . . . . . 216

B.1 Schematic Stress Profile . . . . . . . . . . . . . . . . . 223

C.1 Schematic of two layer model . . . . . . . . . . . . . . 225

C.2 Schematic of layered interface steepening . . . . . . . . . . 229 


\section{List of Tables}

2.1 Width of the Outflow . . . . . . . . . . . . . . 74

2.2 Core Temperature and Salinity Values . . . . . . . . . . . . 78

2.3 Flux of Pure Mediterranean Water . . . . . . . . . . . . . . 98

2.4 Section Averaged Properties of the Mediterranean Outflow . . . . . . 134

3.1 Initial Conditions . . . . . . . . . . . . . . . . . . 152

3.2 Model Sensitivity to Initial Conditions . . . . . . . . . . . . 173

3.3 Model Solution with Different Width Specifications . . . . . . . . . . 191

A.1 Net Volume and Heat Flux . . . . . . . . . . . . . . . . . 215 


\section{Acknowledgements}

I would like to thank my advisor, Dr. Jim Price, for the many years of encouragement and patience. His enthusiasm has taught me the fun of science and I just hope that some of his physical insight and intuition has rubbed off. I would also like to thank my thesis committee, Drs. Paola Rizzoli, Harry Bryden, Micheal McCartney, Larry Pratt, David Chapman and Glen Gawarkiewicz for critically reading the early drafts of this work and providing helpful and stimulating criticisms throughout.

I have received invaluable technical assistance from Maureen Kennelly, Mark Prater and the rest of Tom Sanford's group for the preparation of the data and numerous suggestions. Bud Brown was astonishingly patient redrafting my hand contoured figures in chapter 2. His assistance was invaluable. The Cadiz data were then converted into WHOI/CTD format with the help of Chris Wooding. Lastly, I would like to thank Linda King and the professors at MIT for so generously allowing a wayward WHOI student access to their first rate computer systems at MIT.

I would like to thank Dr. Robert Miller and Dr. Dana Thompson for convincing me to become an oceanographer. Without their encouragement, I would not be here at all.

To my friends, especially Alison Macdonald, Rebecca Schudlich, and Debbie Klatt Barber, a heartfelt thanks for putting up with me. I thank Paul Cloke for his suggestions on the numerical modelling work that never made it to my thesis as well as proof reading this manuscript. Also, my parents John and Jacquelin O'Neil, whom have continually supported me and always taught me that I could accomplish anything. My dad, also for awakening the puzzle solver in me. Lastly and most im-

portantly, I could not have finished this without the love and support of my husband, Warner Baringer. 
In memory of

John Tettemer O'Neil, Jr.

(January 31, 1966 - August 13, 1990) 


\section{Chapter 1}

\section{Introduction}

Evaporation and cooling in closed basins produces some of the densest waters in the world. Overflows from these enclosed marginal seas feed much of this dense water into the open ocean. Overflows are thus critical in determining the deep and intermediate structure of the world's oceans which in turn effects the large scale thermohaline circulation. The outflow from the Mediterranean, which overflows at the Strait of Gibraltar, is thought to have a profound influence on the deep and intermediate thermocline structure of the Atlantic Ocean. A knowledge of how the dense water from the Mediterranean is modified before reaching the open ocean is therefore fundamental to understanding its eventual influence upon the North Atlantic and the global thermohaline circulation.

In this dissertation, the Mediterranean outflow is examined, with the aim of understanding how overflow dynamics and mixing modify the initial characteristics of dense water formed in marginal seas. The Mediterranean is a very convenient overflow to study for reasons we will outline in this chapter, including that the time and space scales are small enough that a reasonably well sampled, synoptic survey of the 
region can be obtained. Also, the enclosed nature of the Mediterranean Sea provides constraints on mass, heat and freshwater entering or leaving the North Atlantic.

High quality data from the Gulf of Cadiz Expedition (GofCExp) are examined to describe and quantify the mixing and dynamics of the outflow. The entrainment rate will be quantified from mass and salt conservation and the implied stresses on the flow will be examined though an analysis of the momentum balance. Several models are also used to interpret these observations. We will concentrate on the vertical and horizontal structure of the Mediterranean outflow after it has exited the Strait of Gibraltar and entered the eastern North Atlantic in the Gulf of Cadiz.

In this introductory chapter, observations and models of the Mediterranean outflow are reviewed to set the stage for this dissertation. First, we discuss the end constraints on the Gulf of Cadiz by reviewing the origins of the Mediterranean outflow and the ultimate intermediate water mass the Mediterranean outflow eventually forms in the North Atlantic. Then, we summarize the observations of the outflow in the

Strait of Gibraltar that provide us with 'upstream' conditions for our main region of interest, the Gulf of Cadiz. Previous surveys of the outflow in the Gulf of Cadiz are described and outstanding questions are noted. Finally, models of the Mediterranean and other similar density currents are presented, which we will later examine to provide insight into the observations.

\subsection{The Mediterranean Sea and Its Influence on the North Atlantic}

The Mediterranean Sea is forced by intense evaporation which creates dense Levantine Intermediate Water (LIW) and Mediterranean Deep Water which exit the Mediterranean through the Strait of Gibraltar. At the Strait of Gibraltar, this dense 
water meets the fresh, light North Atlantic water and creates a two layer exchange flow. This Mediterranean outflow water exits through the Strait of Gibraltar as a dense plume, moving down the northern continental slope of the Gulf of Cadiz. The overflow slowly loses its high salinity as it mixes with the fresh North Atlantic water and spreads northwest along the southern coast of Spain. It becomes neutrally buoyant near Cape St. Vincent with a salinity still 1 pss above western North Atlantic Central Water of similar temperature. Leaving the Coast of Portugal the outflow spreads throughout the North Atlantic at an average depth of $1100 \mathrm{~m}$ in a tongue of high salinity water affecting $(\mathrm{T}, \mathrm{S})$ properties nearly to the western boundary (Worthington, 1976).

The Mediterranean Sea is a semi-enclosed basin often studied because it contains many of the characteristics of the general circulation, including deep and intermediate water formation, jets and eddies and intense air sea interaction. Semienclosed seas also have the advantage that they are isolated so that advection of heat, salt and fresh water into the entire basin can be measured by sampling a limited area, in this case the Strait of Gibraltar (as well as river runoff and exchange with the Black Sea). Heat and fresh water budgets are thus much simpler to formulate and compare with air-sea flux formulae than they are in the open ocean.

Recently, Garrett et al (1993) used the semi-enclosed nature of the Mediterranean to adjust their estimates of solar insolation and evaporation to match the estimated advective heat flux measured in the Strait of Gibraltar (Bethoux, 1979; preliminary results of Macdonald, Candela and Bryden, 1993). The spatial distribution of the average total heat flux for the Mediterranean is shown in figure 1.1 (Garrett et al. 1993). This shows an average heat loss over the entire Mediterranean of $-7 \mathrm{Wm}^{-2}$, consistent with the advective flux of Bethoux, 1979 and Macdonald, Candela and Bryden, 1993. The most significant heat loss to the atmosphere occurs in the northern portion of the Mediterranean Sea, particularly in the Gulfe du 


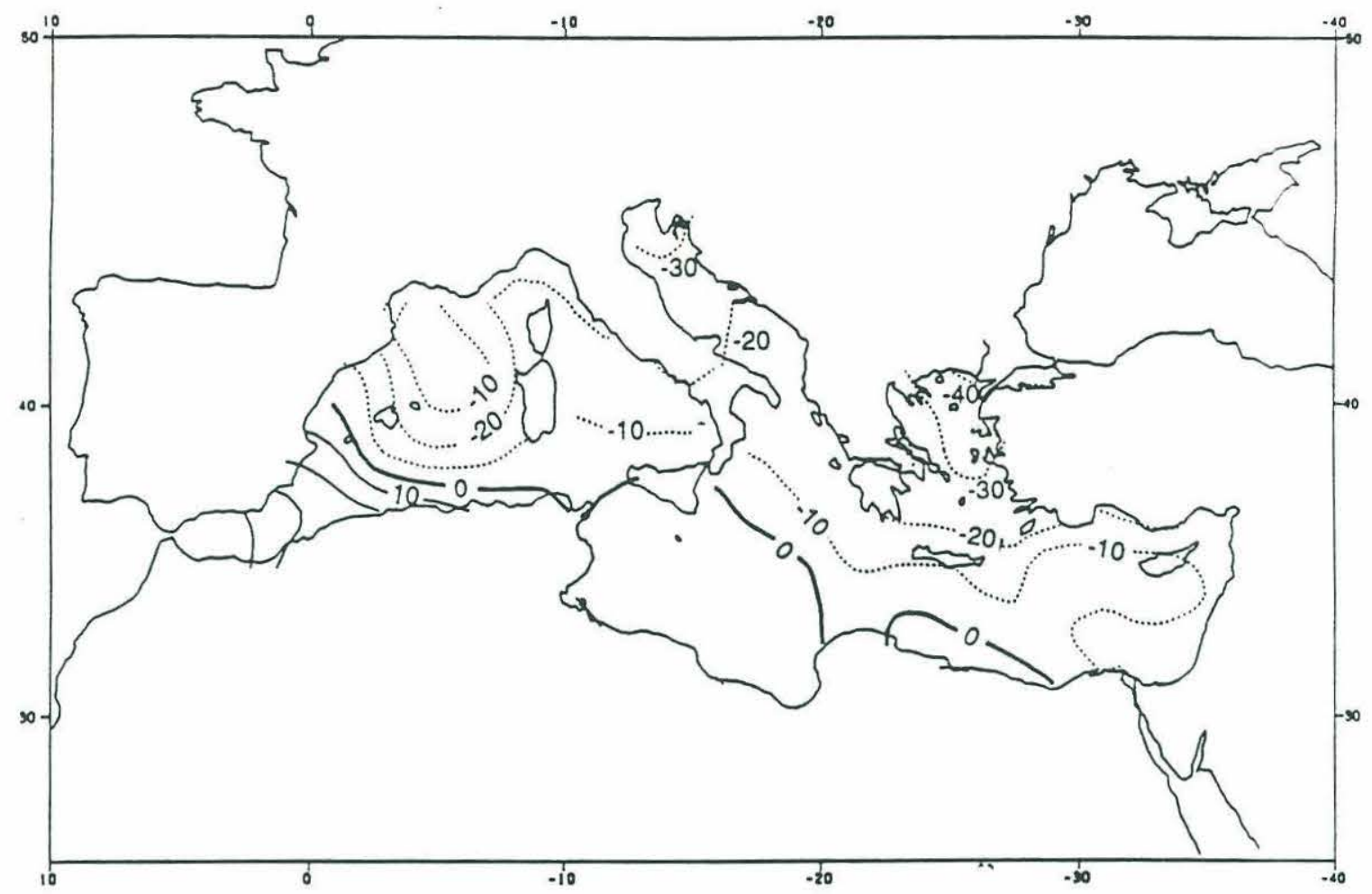

Figure 1.1: Total Heat Flux into the Mediterranean (reproduced from Garrett et al, 1993, their figure 3). Values were adjusted to match the average heat loss over the entire Mediterranean of $7 \mathrm{Wm}^{-2}$ (see Garrett et al, 1993). Contour interval is $10 \mathrm{~W}$ $\mathrm{m}^{-2}$ and heat loss is negative with dashed contours. Heat gain is positive with solid contours.

Lion and the northern Adriatic Sea where deep water forms (Wust, 1961; MEDOC GROUP, 1970; Malanotte-Rizzoli and Hecht, 1988; Schlitzer et al., 1991). Significant heat loss to the atmosphere is also shown in figure 1.1 in the eastern Mediterranean basin where Levantine Intermediate Water (LIW) is formed (Nielsen, 1912; Lacombe and Tchernia, 1960; Wust, 1961; Sur et al., 1992, POEM GROUP, 1992). Deep and intermediate water are formed in these localized areas through this strong air sea interaction.

The general pattern of the circulation of the deep and intermediate waters in the Mediterranean has remained essentially as Wust schematized in figure 1.2 (reproduced from Wust, 1961). Relatively fresh water of Atlantic origin flows in 
through the Strait of Gibraltar and through a series of eddies and jets (Bormans and Garrett, 1989; Millot, 1985, 1987) replaces the water convectively overturned through intense air-sea interaction (Stommel et al., 1971). Deep water produced in the Gulfe du Lion and the Adriatic is shown sinking to fill the deepest portions of the western and eastern Mediterranean basins which are separated by the Strait of Sicily. LIW is formed in the eastern Mediterranean near the Rhodes gyre (Neilsen, 1912; Lacombe and Tchernia, 1960; Ovchinnikov, 1984; Ozturgut, 1976; Sur at al, 1992) and spreads at intermediate depths, near $300 \mathrm{~m}$ in the eastern basin (Lascaratos et al, 1993) and between $200 \mathrm{~m}$ and $800 \mathrm{~m}$ in the western basin (Stommel et al., 1973).

Most of the Mediterranean outflow as it leaves the Mediterranean through the Strait of Gibraltar is composed of the LIW with only $10 \%$ of the outflow consisting of Western Mediterranean Deep Water (WMDW) (Bryden and Stommel, 1984). After the flow exits the strait and the Gulf of Cadiz (see the next section) it enters the eastern North Atlantic at intermediate depths between 600-1500 m (Madelain 1967) with core properties near $1200 \mathrm{~m}$ (Zenk 1975a). The outflow contributes a large salinity tracer into the North Atlantic whose signal can be traced to the Bermuda rise (Armi and Bray 1982). Figure 1.3 shows the salinity on an isopycnal that was chosen to represent the Mediterranean outflow water in the North Atlantic (reproduced from Reid, 1979; his figure 3). High salinity values appear to be spreading into the North Atlantic west-southwest of the Gulf of Cadiz in the so called 'salt tongue' (Arhan, 1987; Worthington, 1976). High salinities also appear along the coast of Portugal where the outflow has turned northward after passing the Iberian Penninsula (Zenk and Armi, 1990; Maillard, 1986). The prominent shape of the outflow tongue has led to many attempts to determine the mid-depth circulation in the Atlantic (Arhan, 1987; Worthington, 1976; Richardson and Mooney, 1975; Needler and Heath, 1975). It is now known that the Mediterranean tongue is populated by many submesoscale coherent vortices or so called Meddies (Richardson et al., 1991). The debated mechanisms for the formation of these Meddies includes flow instability, boundary layer 


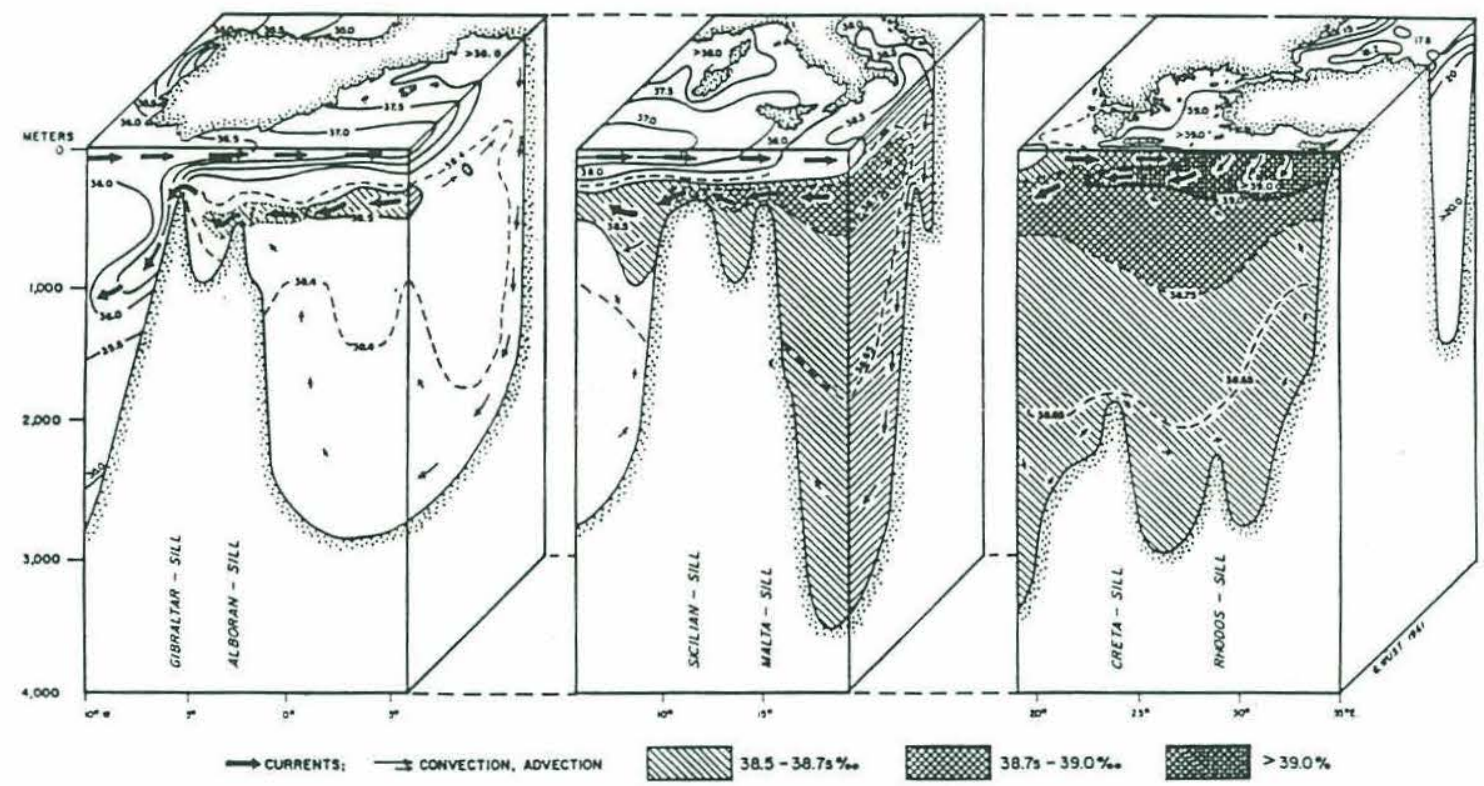

Fig. 8. Schematic block diagram of vertical circulation and distribution of salinity in the Mediterranean Sea during winter.

Figure 1.2: Schematic block diagram of the vertical circulation in the Mediterranean Sea (reproduced from Wust (1961), his figure 8). Salinity contours above 38.5 pss are hatched. 
instability, geostrophic adjustment, outflow transport intermittency, and/or interaction with the Azores front (Griffith et al, 1982; D'Asaro, 1988; McWilliams, 1988; Nof, 1991; Kase and Zenk, 1987). It is not yet known how much of the salinity tracer signal is injected by Meddies, but Bower and Armi are working on it. The effects of the Mediterranean outflow may be far reaching in the global ocean. Reid (1979) postulates that this Mediterranean salt flux preconditions the water that feeds the polar regions and forms deep water. Although the high salinity of the North Atlantic Current may dominate the poleward advection of salt, the Mediterranean must contribute to the overall high salinities in the North Atlantic which in turn are intimately connected to the global thermohaline circulation.

In theories of abyssal circulation (i.e. Stommel and Arons 1960), the circulation is forced by gradual upwelling over the entire ocean interior and localized sinking of cold water at the poles and marginal seas. Deep water masses formed in marginal seas enter the open ocean by spilling over isolating sills. These currents, often called overflows, plumes and density currents, link the fairly local processes which produce deep water with the global circulation. For instance, the Denmark Strait overflow feeds the Deep Western Boundary Current (DWBC) in the North Atlantic which not only effects the deep circulation but has also been shown to effect the separation latitude of the Gulf Stream (Thompson and Schmitz 1989, Hogg and Stommel 1985).

What we know about the origin of the Mediterranean outflow and its effect on the North Atlantic

- The Mediterranean outflow forms an intermediate water mass in the North Atlantic with $\mathrm{T}, \mathrm{S}=\left(12.0^{\circ} \mathrm{C}, 36.5 \mathrm{pss}\right)$ between $800-1300$ meters depth.

- The enclosed nature of the Mediterranean sea allows one to make strong conservation statements about the net exchange with the North Atlantic. 


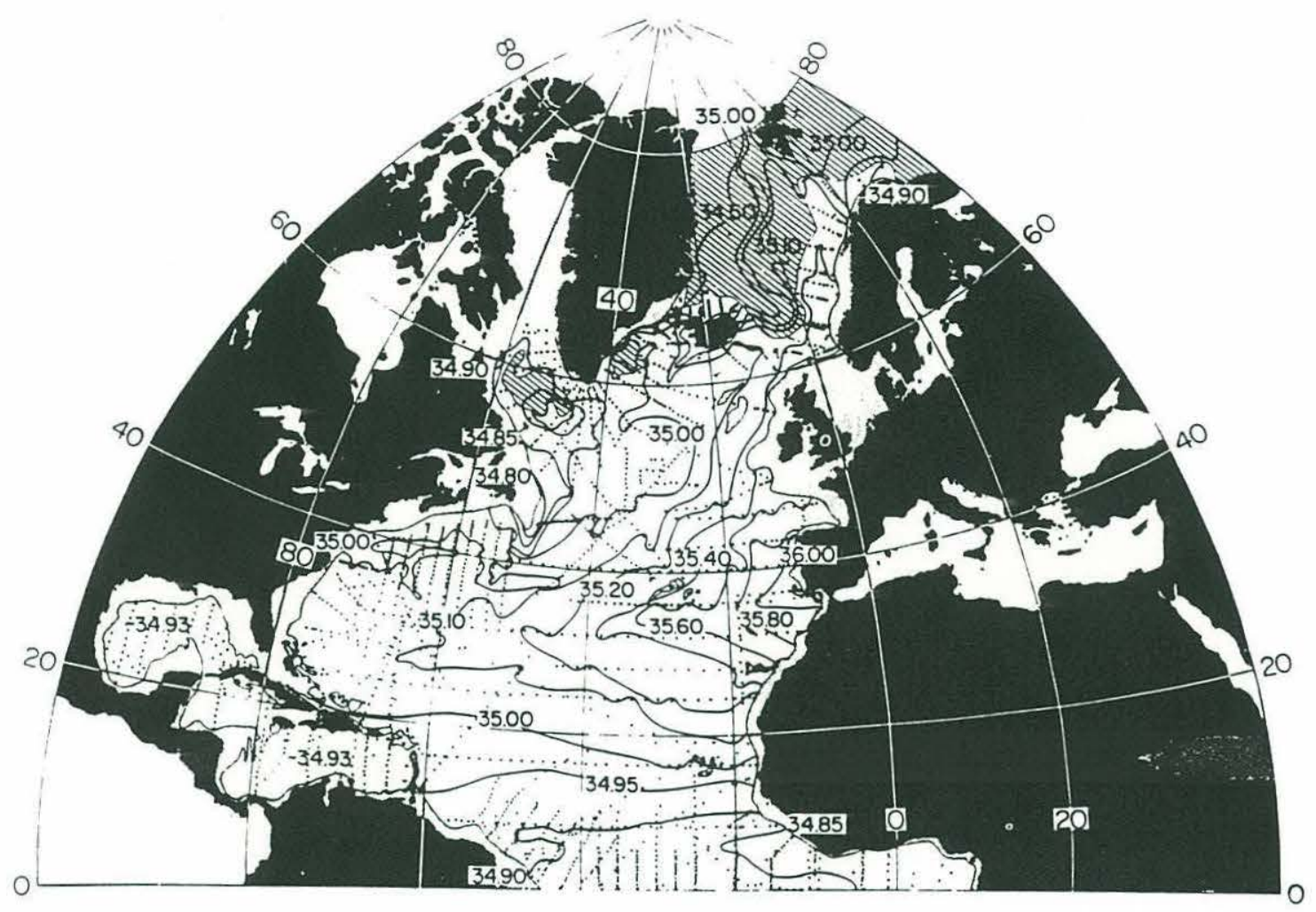

Figure 1.3: Salinity along an isopycnal chosen to represent the Mediterranean outflow in the North Atlantic (reproduced from Reid, 1979). The isopycnal was defined as $\sigma_{1}=32.274$ below $500 \mathrm{~m}$ and as $\sigma_{0}=27.69$ above $500 \mathrm{~m}$. This isopycnal is near 1200 meters deep near the Gulf of Cadiz and surfaces in northern latitudes where hatched surface salinity values are shown.

\section{Outstanding Questions}

- How sensitive are the temperature, salinity and mass flux of the mixed Mediterranean water to the conditions at the Strait of Gibraltar? Why does it separate at mid-depths when the source water density is so large? 
- How much of the mixed Mediterranean water is of Mediterranean origin? Given this large source of water entering the Atlantic, where is the sink of water that conserves mass?

\subsection{Observations of the Mediterranean Outflow in the Strait of Gibraltar}

Since the time of Neilsen (1912), simple mass and salt conservation statements have been applied to the strait to estimate the exchange between the Mediterranean and the Atlantic. These conservation equations, called the Knudsen relations, relate the exchange to the salinity difference and the net evaporation over the Mediterranean. Neilsen (1912) first applied these relations and estimated an inflow and outflow transport of 1.88 and $-1.79 \mathrm{~Sv}\left(1 \mathrm{~Sv}=10^{6} \mathrm{~m}^{3} / \mathrm{s}\right)$ respectively.

In the decades that followed, many other investigators repeated this using revised evaporation estimates and obtained slightly lower values (Sverdrup et al., 1942; Defant, 1961; Bethoux, 1979; Lacombe et al., 1981). The most commonly quoted transport is that estimate of Lacombe and Richez (1982) which reduces the exchange to $1.2 \mathrm{~Sv}$. Outflow transport estimates have ranged in values between 0.9 Sv to $1.8 \mathrm{~Sv}$ (summarized in Hopkins, 1978). Recently Bryden, Brady and Pillsbury (1989) (Bryden and Kinder, 1992) used current meter measurements from the recent Gibraltar Experiment to calculated a time averaged outflow transport of $0.76 \mathrm{~Sv}$ and inflow transport of $1.04 \mathrm{~Sv}$. From a longer time series, more recent estimates from the Gibraltar Experiment reduce these mean exchange values further to 0.68 and 0.72 Sv (Bryden, Candela and Kinder, 1993). ${ }^{1}$

\footnotetext{
${ }^{1}$ We regard the recent transport measurements as more refined and accurate, and we do not think the apparent decrease in the estimated exchange is indicative of a true change in the exchange (agreed by Bryden, Candela and Kinder, 1993).
} 
The resurgence of interest in the Gibraltar region, including the recent yearlong survey from October 1985-October 1986 called the Gibraltar Experiment (Kinder and Bryden, 1987), has improved our estimates of the mean exchange but also raised questions about the time dependent nature of the outflow. The Gibraltar Experiment showed that there is a semidiurnal tide in the strait which is so strong that it can reverse the flow at all depths (Bryden, Brady and Pillsbury, 1989). Figure 1.4 shows the time series of the along strait velocity from several depths at mooring 2 from the Gibraltar Experiment (reproduced from Bryden, Candela and Kinder (1993), their figure 4) where the diurnal tide and fortnight spring/neap tides are apparent. The instantaneaous transports for the M2-tide have amplitudes of $2.3 \mathrm{~Sv}$ and $1.3 \mathrm{~Sv}$ for the inflow and outflow respectively (defining the interface between Atlantic inflow and the Mediterranean outflow by the 37.0 pss isohaline). Bryden, Candela, and Kinder (1993) also find that this strong tidal signal is correlated and in phase with vertical displacements of the 37.0 pss isohaline and this correlation contributes half of the time averaged outflow of Mediterranean water. The undulation of the interface also leads to term bursts of inflow and outflow transport as large as -0.21 and $0.17 \mathrm{~Sv}$ respectively (Candela et al., 1990). In fact, subinertial barotropic flows also contribute pulses of exchange with $0.4 \mathrm{~Sv}$ rms transport due principally to atmospheric pressure forcing in the Mediterranean. These subinertial flows can also slightly influence the mean exchange (but this effect is less than $10 \%$ of the tidal contribution; Candela et al., 1989).

Early hydraulic theories such as Bryden and Stommel (1984), Stommel and Farmer (1953) and Whitehead et al. (1974), assume the flow is steady. More complex hydraulic theory was extended to include friction, multiple control points, and barotropic flow to simulate the strong tides in the strait (Armi and Farmer, 1986, 1987; Pratt, 1986; Dalziel, 1988, 1990; Bormans and Garrett, 1989). Armi and Farmer (1988) discuss in detail the along-strait structure of the outflow/inflow and use the ideas of quasi-steady hydraulic theory to interpret their extensive set of measure- 


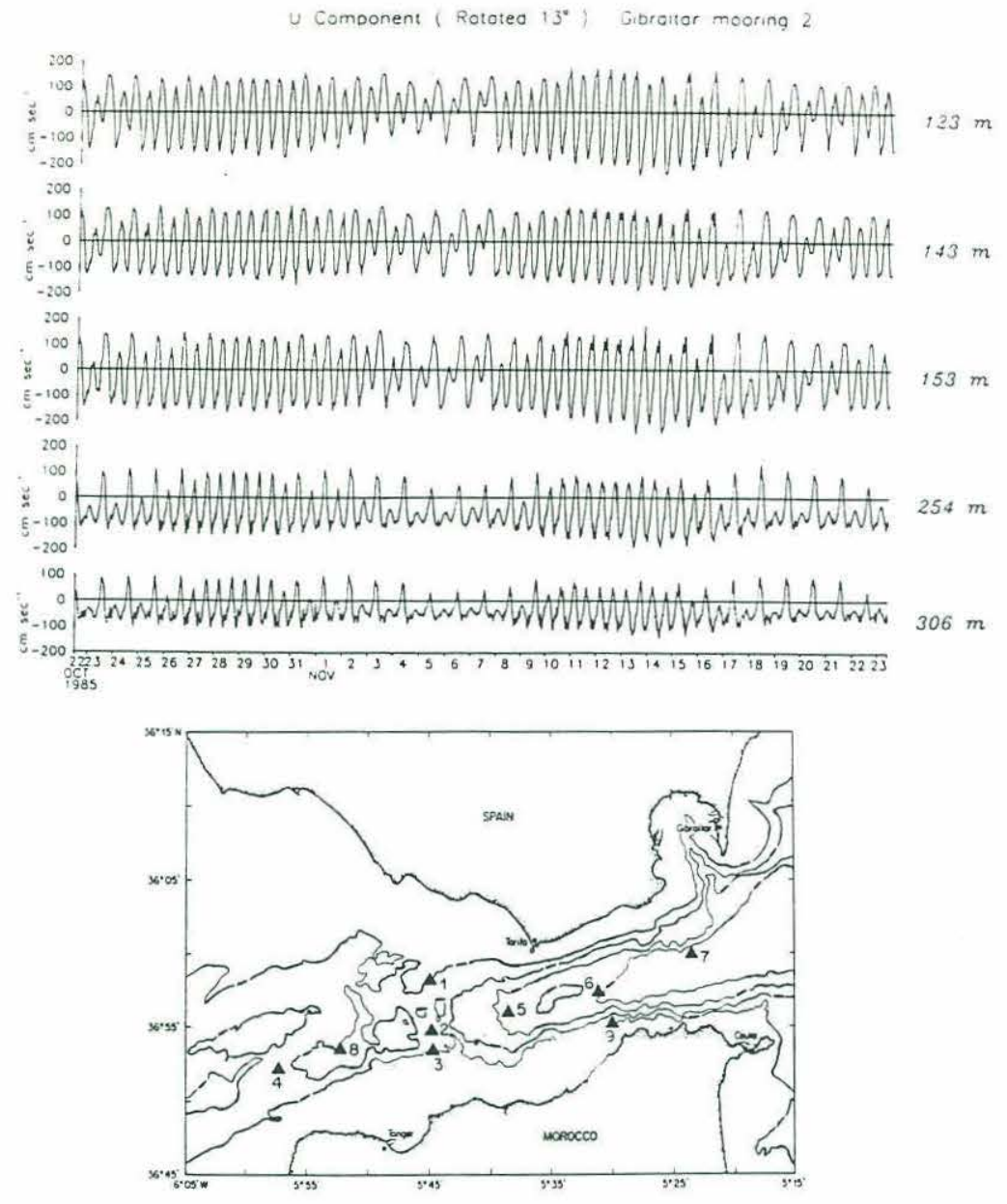

Figure 1.4: Time series of along Strait of Gibraltar (u, or east-west) velocity reproduced from Bryden, Candela and Kinder (1993, their figure 4). The current meter is located at the Camarinal sill, mooring number 2 shown in map below. Positive velocity is directed to the east, into the Mediterranean. The diurnal M2 tide reverses the flow at all depth. A fortnightly modulation of the spring/neap tides is also apparent.

ments obtained from expendable profilers and acoustic methods during the Gibraltar Experiment. Figure 1.5 shows a summary schematic of their analysis of the interface depth and the time dependent hydraulic response during different stages of the spring tide.

Armi and Farmer (1988) indicate that the flow is always critically controlled at the Spartel West sill and that the interface executes considerable vertical excursions during a tidal cycle which implies that the Tangier basin is filled and drained but 


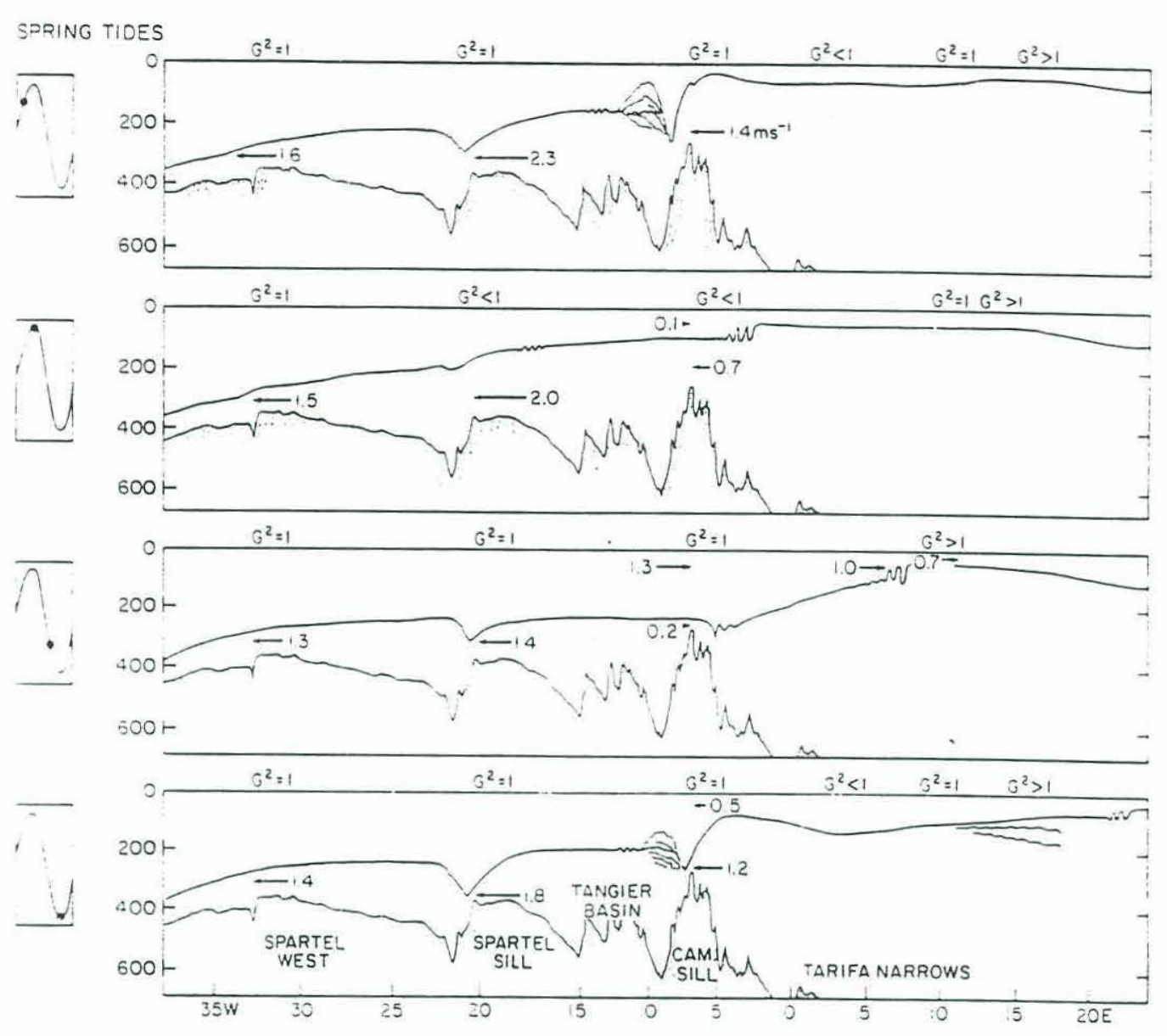

Figure 1.5: Schematic diagram summarizing the time dependent hydraulic response in the Strait of Gibraltar reproduced from Armi and Farmer, 1988. Stage of the spring tide shown on the left. G is a composite Froude number which indicates internal control when $G^{2}=1$.

controlled at Spartel West. Although the flow reverses at the sill, the flow has less than a $20 \%$ tidal variation on top of a mean speed of $1.4 \mathrm{~m} / \mathrm{s}$ at Spartel West $(25 \mathrm{~m}$ above the bottom; Armi and Farmer, 1988). Candela et al. (1990) confirm that the correlations between the outflow currents and interface depth etc., drop to vanishingly small values away from the Camarinal Sill (Macdonald, Candela and Bryden, 1993; Candela et al., 1990, Candela, 1991). Therefore, although time dependent fluctuations at inertial and subinertial frequencies contribute significantly to the total mass, heat and sait fiux at the sill, they reduce their "contribution to the mean fluxes to negligible values' (Macdonald, Candela and Bryden, 1993) to the east and west of the sill. 
What we know about the Mediterranean outflow in the Strait of Gibraltar

- The mean transport of Mediterranean outflow is about 0.7 Sv at the Camarinal sill and typical core properties are $(\mathrm{T}, \mathrm{S})=\left(13.0^{\circ} \mathrm{C}, 38.4 \mathrm{pss}\right)$.

- There is slightly more water flowing from the Atlantic into the Mediterranean to balance the evaporation over the Mediterranean.

- There are very large tides, predominantly semi-diurnal, in the Strait of Gibraltar and large interface displacements correlated with the tides lead to time dependent exchange at the Camarinal sill.

- East and west of the sill interface fluctuations are not correlated with the tides, and downstream of Spartel West sill, the time dependent fluctuations in speed are less than $20 \%$ of the mean.

\subsection{Observations of the Mediterranean Outflow in the Gulf of Cadiz}

Many surveys show the high salinity Mediterranean outflow leaving the Strait of Gibraltar and spreading along the northern continental slope in the Gulf of Cadiz (Madelain, 1970; Ambar and Howe, 1979a, 1979b; Ochoa and Bray, 1991). Figure 1.6 shows the path of the outflow as depicted by Madelain (1970).

The northward turn of the outflow has been described as an effect of the strong topographic steering of the flow (Ochoa and Bray, 1991; Madelain, 1970), or alternatively a deflection of the flow due to the Coriolis force (Ambar and Howe, 1979a; Price et al., 1993). Kenyon and Belderson (1973) believe the north-south ridge discussed in Ochoa and Bray (1991) is actually depositional in nature and is thus an artifact of sediment transported by the outflow over very long time scales and could represent 


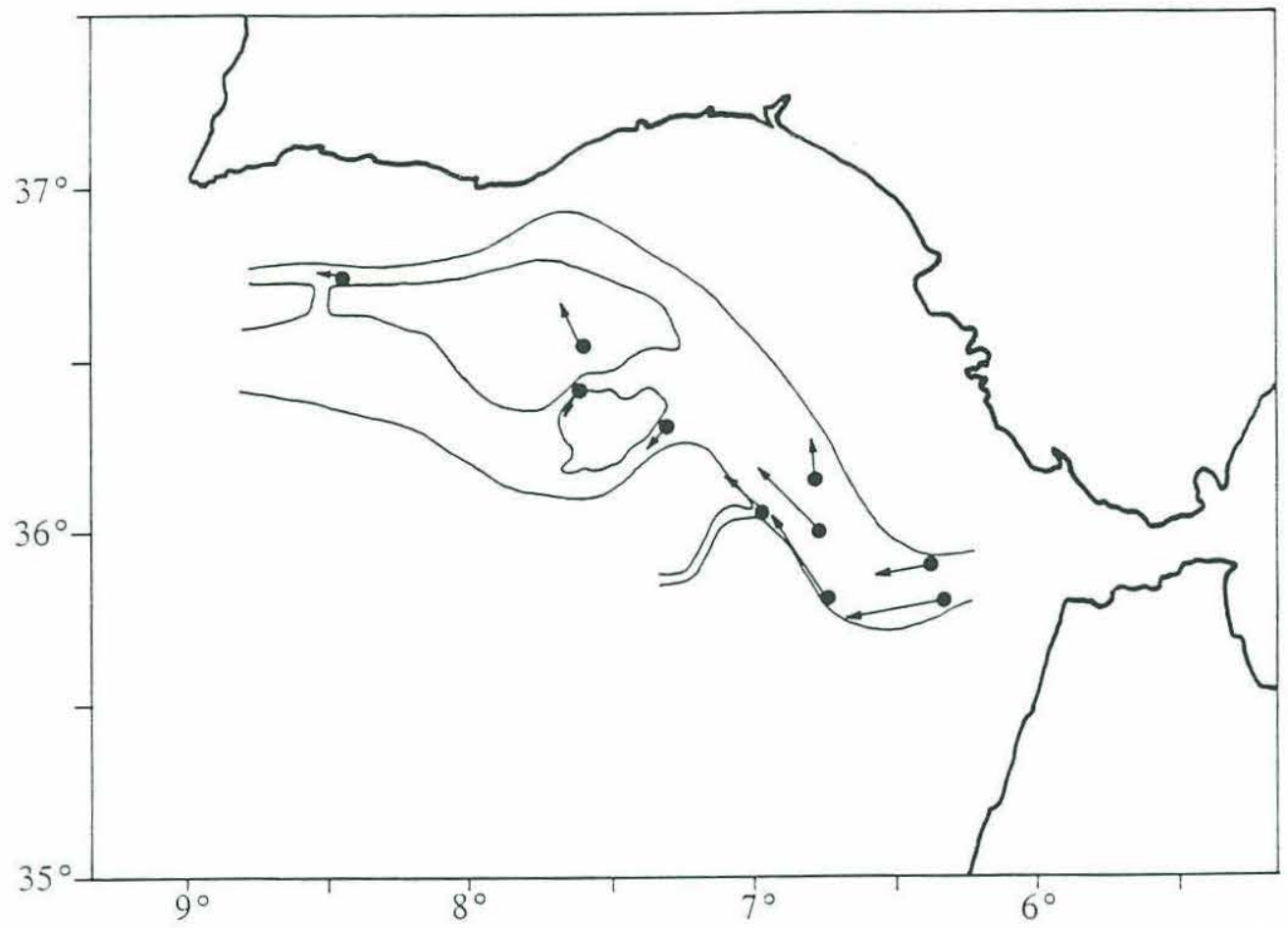

Figure 1.6: Schematic of subdivision of the Mediterranean outflow in the Gulf of Cadiz reproduced from Madelain (1970).

variations in the outflow strength. The outflow is certainly strongly influenced by the topography in the Gulf of Cadiz (Heezen and Johnson, 1969; Madelain, 1970).

One illustration of the importance of topography has been the identification of two separate cores of outflow separating into the North Atlantic near 700-800 m and 1000-1200 m (Ambar and Howe, 1979a). Madelain (1970) believed that the complex topography of the region (shown in figure 1.7) was responsible for the subdivision of the flow into two cores. Zenk (1975a) clearly identified two distinct water types present in the open ocean eastern North Atlantic which he suggests result from tidal mixing within the Strait of Gibraltar (Seidler, 1968; Howe et al., 1974). Ambar and Howe (1979a), on the other hand, propose that the upper Mediterranean water core could be formed through the mixing with warmer North Atlantic Central Water (NACW). Ambar and Howe (1979b) later identified a third, shallower core near $7^{\circ} \mathrm{W}$ between $400-600 \mathrm{~m}$ (Zenk, 1975a). This shallower core maintains its $\theta / S$ identity as 


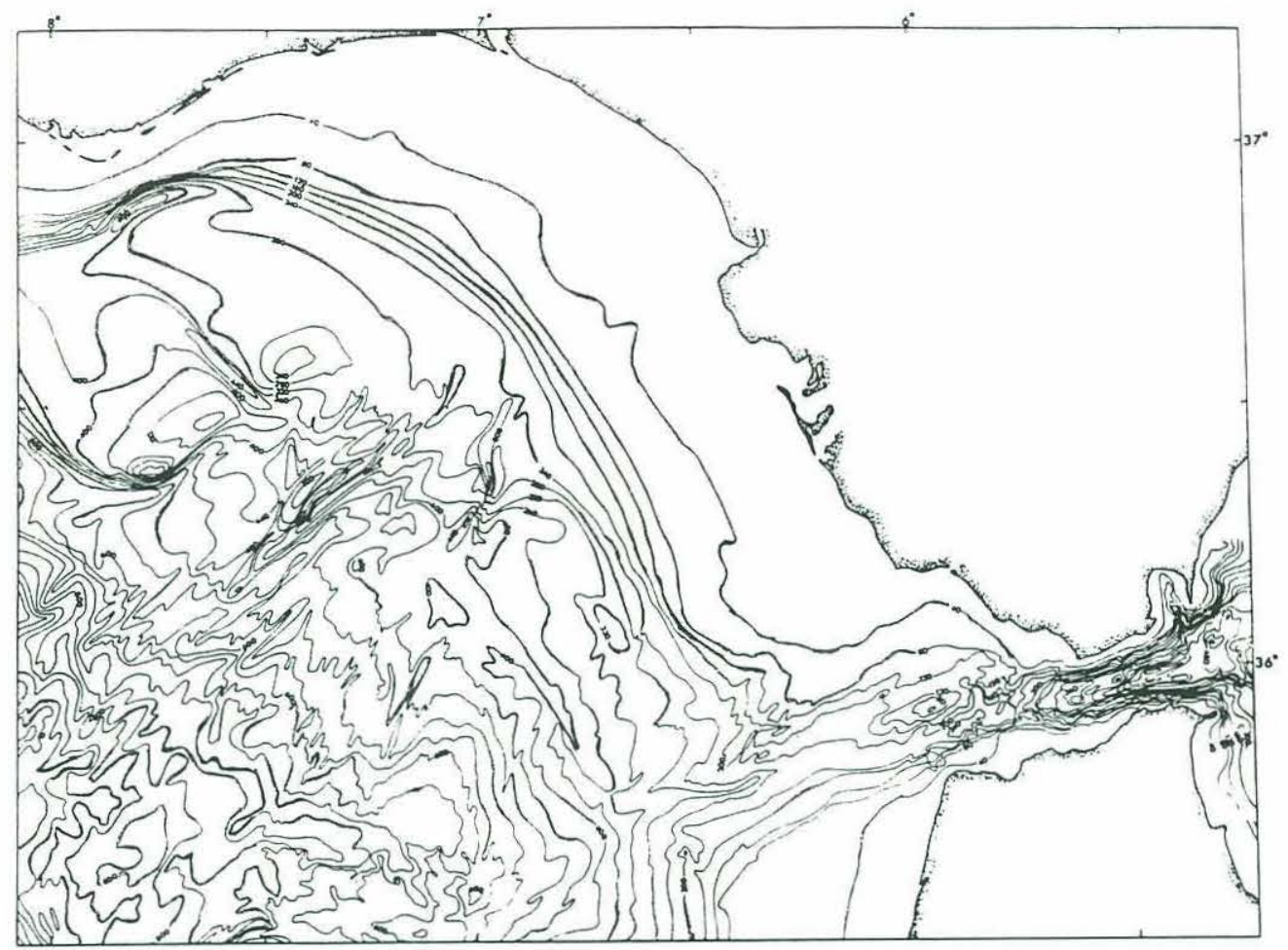

Figure 1.7: Gulf of Cadiz Bathymetry reproduced from Kenyon and Belderson 1973. Contour interval of 40 fathoms.

the outflow turns to move northward along the coast of Portugal (Ambar, 1983) and is thought to originate from winter mixed layers on the northern shelf in the Gulf of Cadiz (Ambar and Howe, 1979b)

As much as the Gulf of Cadiz has been studied, until recently few transport and property flux estimates were found in the literature. Ambar and Howe (1979b) estimate an outflow transport of $1 \mathrm{~Sv}$ near the strait and $3 \mathrm{~Sv}$ near Cape St. Vincent (Ambar and Howe, 1979b). More recently, Ochoa and Bray (1991) estimate a net flux of Mediterranean water of $0.5 \mathrm{~Sv}$ near the strait and $2.2 \mathrm{~Sv}$ near $7^{\circ} 30^{\prime} \mathrm{W}$ and $8^{\circ} \mathrm{W}$. Using a mixing model of tracer data, Rhein and Hinrichsen (1993) estimate the Mediterranean outflow transport reaching a maximum near $8^{\circ} \mathrm{W}$ of $3.7 \mathrm{~Sv}$ by assuming an outflow of $1 \mathrm{~Sv}$ at the strait. 
Prior to the Gibraltar Experiment, direct velocity measurements of the outflow have been sparse in space and short in duration. Maximum current speeds of 150 $\mathrm{cm} / \mathrm{s}$ in the strait and a decrease to $85 \mathrm{~cm} / \mathrm{s} 40 \mathrm{~km}$ downstream have been observed (Heezen and Johnson 1969, Ambar and Howe 1979b, Madelain 1970). A $50 \mathrm{~cm} / \mathrm{s} \mathrm{semi-}$ diurnal modulation in the surface water and about half that in the plume have been observed (Grundlingh 1981). Stanton (1983) found low frequency variability in the outflow west of the sills occurring with the same time scale as extreme meteorological conditions (i.e. 3-5 days). Grundlingh (1981) examines a solitary increase in current amplitude lasting 2-4 days that coincided with extreme weather conditions. Johnson et al. (1993a) found little variability in the outflow throughout one tidal cycle using expendable shear probes.

\section{What we know about the Mediterranean outflow in the Gulf of Cadiz}

- The Mediterranean outflow follows the northern continental slope of the Gulf of Cadiz along a path that has remained essentially unchanged for decades.

- The outflow develops substantial horizontal structure, at one point splitting into as many as three branches.

- Somewhere in the Gulf of Cadiz, the outflow increases its transport from less than $1 \mathrm{~Sv}$ at the strait to 2.2-3.0 Sv near Cape St. Vincent by entraining North Atlantic Central Water.

\section{Outstanding Questions}

- Is the outflow channeled by the topography or is the flow strongly constrained by inertia to bank rapidly against the continental slope?

- How are two cores generated? How does the flow spread? 
- Is the mixing localized or gradual? Is the observed entrainment correlated with low Richardson numbers?

- What are the major force balances governing the evolution of the plume? What makes the flow descend at all?

\subsection{Models of the Mediterranean Outflow and other Density Currents}

Smith (1975) formulated a streamtube model which includes bottom friction by means of a quadratic drag law and an entrainment rate proportional to the velocity, with both of these coefficients estimated diagnostically by finding the best fit to the

observations. From continuity, the divergence of the along-stream transport must be equal to the entrainment rate, while temperature and salinity are diluted by the water entrained into the plume. The flow is driven by a pressure head and retarded by friction. The cross stream geostrophic balance is modified by the curvature of the flow along a uniformly sloping bottom. The unknown drag coefficient and entrainment constant were determined by tuning the model to data.

The ratio of drag to entrainment governs the behavior of the model solution; when drag dominates, the current slides down the continental slope while maintaining its initial T/S structure. When entrainment dominates, the density anomaly is eroded and the net driving force down the slope will be reduced. The ratio of entrainment versus bottom stress then determines whether an overflow settles at intermediate depths or falls to abyssal depths.

This model has some obvious limitations which Smith (1975) pointed out. The model uses only integral flow properties and tells us only about the evolution of the 
cross sectional area of the outflow. As a consequence, the entrainment is proportional only to the flow speed and not to the width over which mixing takes place. Smith's entrainment constants vary from $1 \cdot 10^{-2}$ in the Mediterranean outflow to $15 \cdot 10^{-2}$ in the Denmark Strait outflow with little physical justification. In many ways applying Smith's model is similar to estimating eddy diffusion coefficients from data; based on the assumed law governing diffusion, friction, or entrainment the best coefficient is determined by comparing the model output to data. These limitations provide little insight into the dynamics governing the flow. The solution overemphasizes the entrainment into the Mediterranean outflow: he finds that the entrainment dominates the bottom stress far downstream which forces the flow to entrain over $15 \mathrm{~Sv}$ before it enters the North Atlantic.

Killworth (1977) makes entrainment proportional to flow speed and the width over which entrainment takes place. The bottom stress is then applied to a surface instead of acting as a body force as in Smith's model. Now however, the height and the width of the plume must be determined by the model. Continuity gives one constraint for the height or width and Killworth assumes the aspect ratio of the height to the width is constant to get another. This ad hoc assumption is unfortunately unrepresentative of the Mediterranean overflow; the height remains virtually constant while the width grows by an order of magnitude.

Killworth examines the flow off the Weddell Sea continental shelf with three models: Smith's three-dimensional model, a two-dimensional analog to Smith's model, and a time dependent non-rotating plume model (Turner 1973). He concludes that only with the addition of a nonlinear equation of state and a depth dependent coefficient of thermal expansion can these models allow the plume to fall to observed depths. For flow moving through large vertical displacements the coefficient of thermal expansion increases; for a given $\mathrm{T}$, the density anomaly increases with depth. 
This gives the plume an effective internal energy source that can carry it farther down the slope.

Pedersen (1980) was the first to attempt to include a variable entrainment. He examines experimental data to define a correlation function between entrainment and bottom slope. Using this new entrainment function reduces the unknown coefficients in the model to the bottom drag coefficient $\mathrm{Cd}$, but it also directly links the entrainment to the bottom drag.

Price and Baringer (1993) and Baringer and Price (1990) further refine plume model dynamics by including a normalized entrainment parameterization based on a Froude number (equivalently a bulk Richardson number following Price, 1979 and Ellison and Turner, 1959). Price and Baringer's (hereafter PB93) model consists of a homogeneous bottom layer, representing the plume, flowing over variable, twodimensional topography and overlain by a continuously stratified, inactive upper ocean. The model includes rotation, mixing into the plume, and quadratic bottom friction. The model equations are integrated along the stream axis to yield unique velocity, temperature, salinity and path for each model realization. This model will be used to simulate the Mediterranean outflow (chapter 3). We will compare the property evolution and the dynamical balances to the GofCExp and we will invert potential vorticity to replace the highly idealized width specification.

A connection between overflows and the general circulation was recently attempted by Speer and Tziperman (1990) using Smith's model. Speer and Tziperman examine the non-local effects induced by overflows by linking Smith's model to the upstream basin by a buoyancy flux lost to the atmosphere, and to the ocean interior through simple linear vorticity dynamics which leads to baroclinic deep flow in the open ocean. In fact, few rotating descending plume models have been developed. The models all treat the plume as homogeneous and ignore or artificially impose the circulation external to the plume. 


\section{What we know about overflow models}

- Diagnostic models that infer bottom friction and entrainment by fitting to data have been developed and applied to several overflows.

\section{Outstanding Questions}

- Can we develop and validate a model that predicts the dynamics of overflows including entrainment and friction that does not require tuning for each overflow?

- Does the simple Price and Baringer (1993) model shed light on the dynamics and force balances of the Mediterranean outflow?

- Can potential vorticity be used as a constraint on this model to improve the dynamics of its broadening?

\subsection{Outline}

The main goal of this work is to examine the dynamics of the Mediterranean outflow. To do this, we have analyzed observations gathered during the Gulf of Cadiz Expedition in October, 1988 (GofCExp). The advantages of these data is that they are of very high resolution, they focus on the initial descent of the outflow and they include in situ shear measurements that provide us with accurate velocity measurements where traditional hydrographic geostrophic calculations fail. In this

thesis several specific questions are addressed including the questions outlined in the previous sections. Does the GofCExp data provide a different picture of the outflow than historical hydrographic surveys? Is the mixing in the outflow uniform or localized? What parameterization of this mixing is appropriate? What are the 
dynamical balances? Does the PB93 model predict the temperature and salinity evolution of the outflow?

In chapter 2 we describe the observations, and examine the horizontal spreading of the outflow, the downstream evolution of its properties, the stability of the flow, and the dynamical balances. In chapter 3 we examine the PB93 model as applied to the Mediterranean outflow and compare its evolution and dynamics with the data described in chapter 2. Finally, in chapter 4 we summarize our results, their implications and their limitations. We also discuss possibilities for further research, in particular, the feasibility of modeling overflows in three dimensional models. 


\section{Chapter 2}

\section{Gulf of Cadiz Expedition}

This chapter examines in detail the structure of the Mediterranean outflow as determined from the Gulf of Cadiz Expedition (GofCExp) beginning with a description of the program in section 2.1. Section 2.2 examines the structure of the flow through the Salinity and Temperature sections obtained on the cruise. The absolute flow field is determined in section 2.3 and the horizontal spreading of the flow and cross stream structure is analyzed in section 2.4. To understand how the Mediterranean is modified to form an interflow in the North Atlantic we quantify the entrainment into the outflow by examining the downstream evolution of the property fluxes. The dominant modification of the outflow is found to occur in a limited area very close to the strait. One hypothesis for this vigorous intermittent entrainment is that the flow is unstable in a Kelvin Holmholtz sense. So in section 2.5 we also address the

questions: Is the flow unstable and is there a Richardson Number dependence to the intermittent entrainment observed? Finally, in section 2.6, the dominant forces governing the evolution of the outflow are determined through an analysis of the momentum budget. 


\subsection{The Gulf of Cadiz Field Program}

In September 1988, the Gulf of Cadiz Expedition studied the waters of the Gulf of Cadiz region and the region surrounding the Ampere seamount. This chapter focuses on the Mediterranean outflow in the eastern Gulf of Cadiz using data collected on the second leg of the expedition. The data to be discussed includes 98 CTD stations obtained with a Sea-Bird electronics CTD and 47 in situ horizontal current profiles gathered with the XCP (expendable current profiler) developed by Thomas B. Sanford at the Applied Physics Laboratory. CTD station locations were taken in the northeastern Gulf of Cadiz between the $200 \mathrm{~m}$ isobath and the $1500 \mathrm{~m}$ isobath in order to sample the entire Mediterranean outflow (figure 2.1). The CTD stations form eight sections $(\mathrm{A}-\mathrm{H})$ normal to the outflow and perpendicular to the local topography, as well as one section along the axis of the Strait of Gibraltar (section I). XCPs were obtained at Sections A through F whenever a CTD cast indicated the presence of salty Mediterranean water. Figure 2.2 shows the locations of these XCP stations. XDPs (expendable dissipation probes) designed by Rolf Lueck measured the rate of turbulent dissipation at selected stations. A brief description of the data processing is given below; fuller descriptions of the data and processing techniques can be found in Kennelly et al.(1989a), Kennelly et al.(1989b), Kennelly et al.(1989c) and Lynch and Lueck (1989).

Kennelly et al (1989a) from the University of Washington performed most of the pre and post cruise data processing and calibration. Water bottle samples were taken in regions of low salinity gradients and used to correct the Seabird conductivity measurements. XCPs and XDPs also measure temperature which allows us to relate the probe measurements to the $\mathrm{CTD}$ profiles. XCP fall rates were adjusted by comparison to concurrent CTD stations temperature profiles (Prater 1991). During the cruise, the XCPs were usually deployed within 30 minutes of the corresponding 


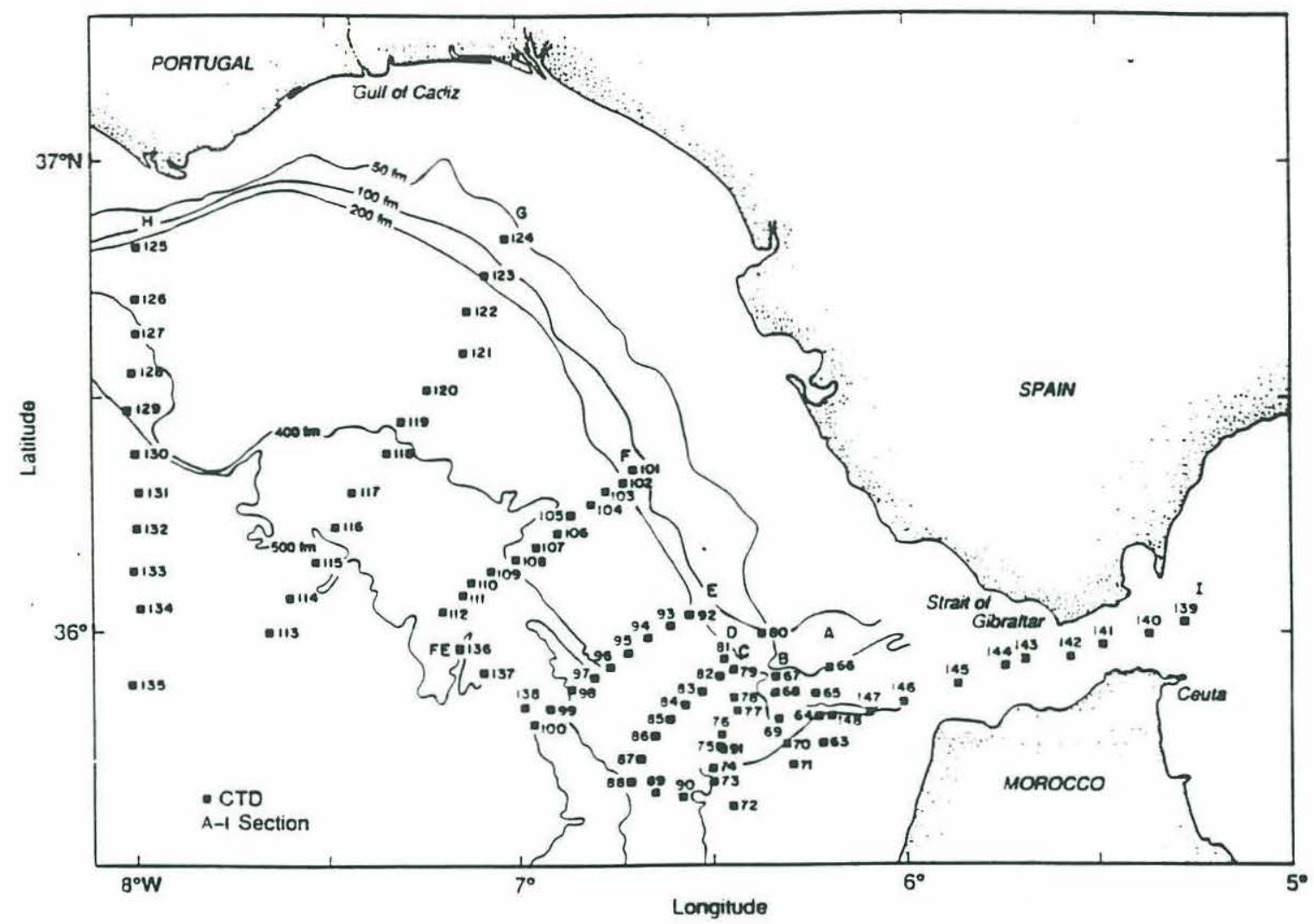

Figure 2.1: Station plan of CTD section locations reproduced from Kennelly et al, $1989 \mathrm{~b}$

CTD cast. CTD stations and XCP stations were considered concurrent if they were within 1 nautical mile and 30 minutes of the CTD cast. The CTD data were averaged over a $2 \mathrm{db}$ interval for pressure, temperature and salinity and the XCP data were averaged for pressure, temperature and $\mathrm{u}, \mathrm{v}$ velocities. Some additional processing was required to remove bad data points near the surface and to replace them with interpolated values. Missed pressure levels or odd pressure levels (i.e. not even) were also interpolated to the regular $2 \mathrm{db}$ spacing. The surface values, particularly for the $\mathrm{XCP}$ profiles, were unreliable because the probe must adjust to a uniform rotation rate. Hence, the $10 \mathrm{db}$ value was used as a constant to the surface. The XCPs yield vertical profiles of data to within $\frac{2}{3} \mathrm{~m}$ to the bottom (Johnson et al., 1993). The $\mathrm{XCP}$ velocities were converted from magnetic coordinates to geographic coordinates. Salinity was calculated by Kennelly et al. (1989b) using the Seabird CTD pressure, 


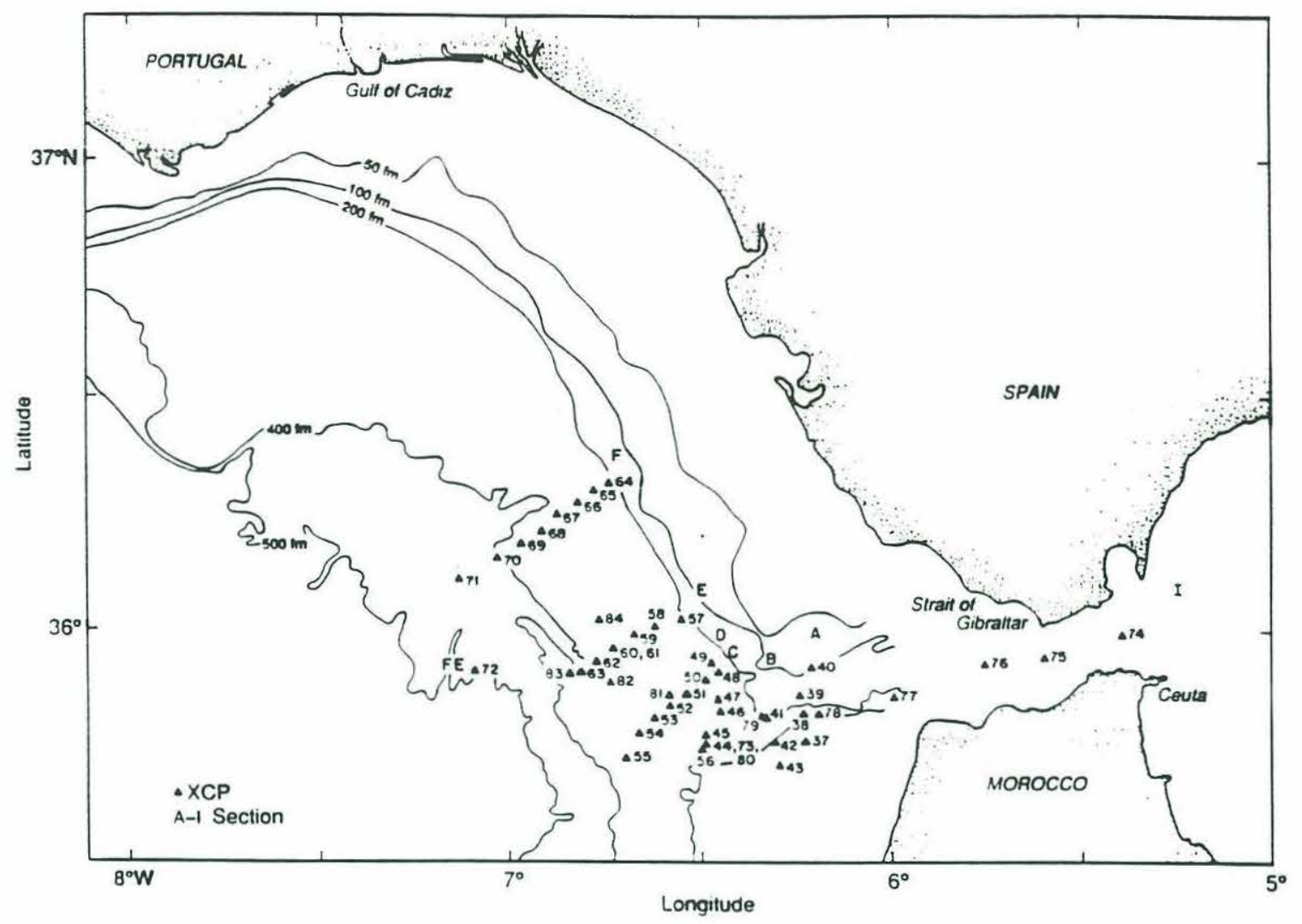

Figure 2.2: Station plan of XCP section locations reproduced from Kennelly et al, $1989 \mathrm{c}$

temperature and conductivity sensors and the method of Perkin and Lewis (1980). Potential temperature and potential density were calculated using EOS80.

\subsection{Hydrographic and XCP Sections}

Nine hydrographic sections span the width of the outflow and are oriented perpendicular to the historical path of the outflow (figure 2.1). These sections span the first $150 \mathrm{~km}$ of the outflow's path in the Gulf of Cadiz, but particularly emphasize the initial 50 kilometers of the outflow path where mixing was expected to be most intense. From these sections, property plots were contoured and shown in figures 2.3, 2.4 and 2.5 . 


\section{SALINITY}

SECTION A

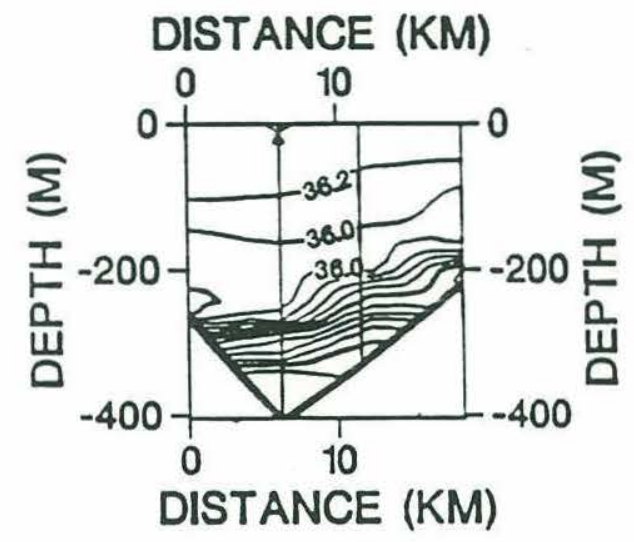

SECTION B

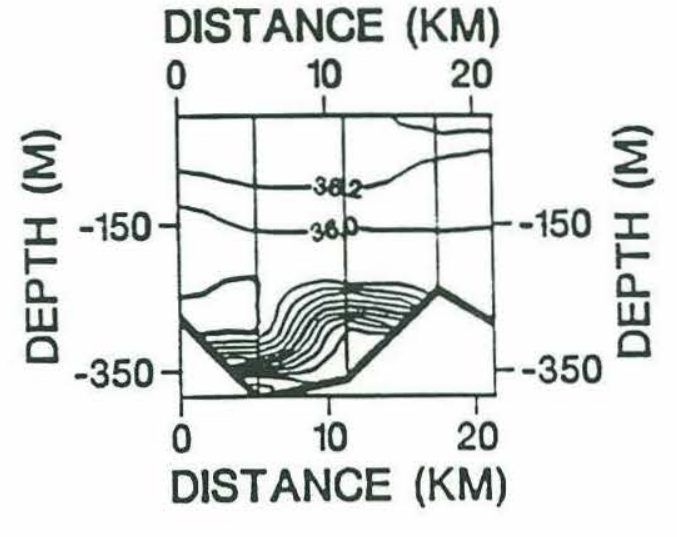

\section{SECTION C}

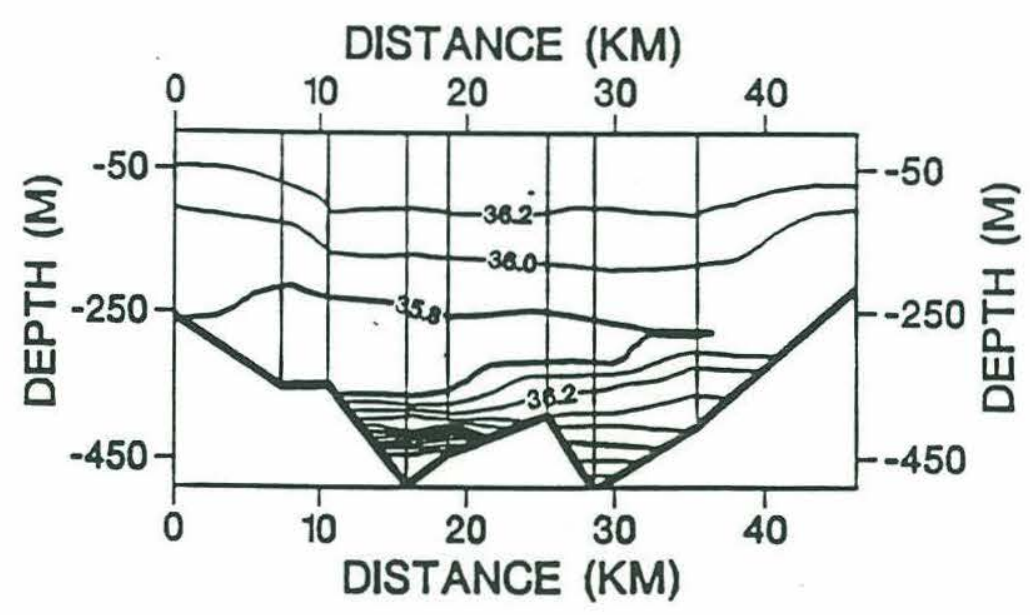

Figure 2.3: Salinity contours along distance versus depth section plots (in pss). (a) Section A, B and C 


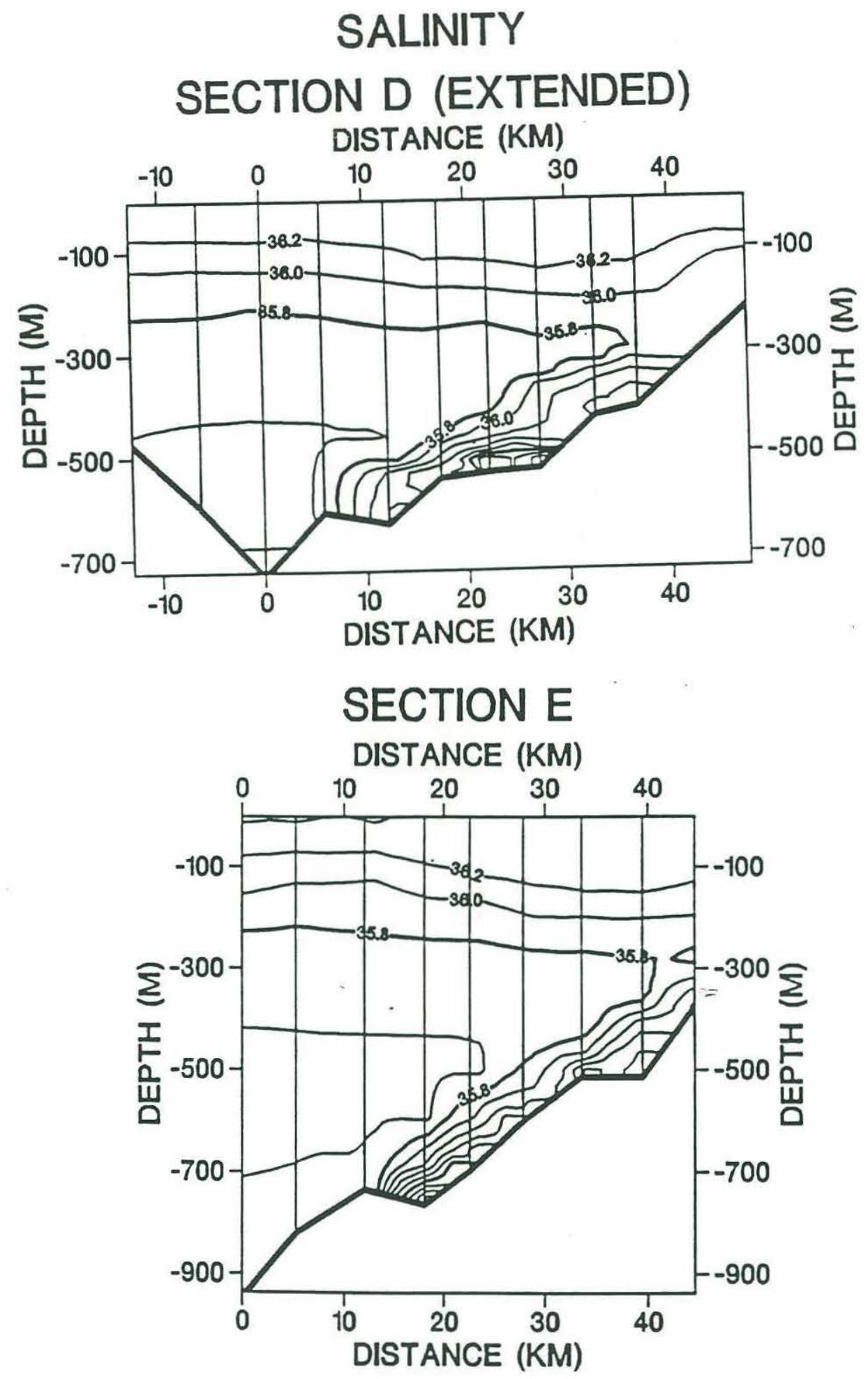

Figure 2.3: (c) Section D and E 


\section{SECTION FE}
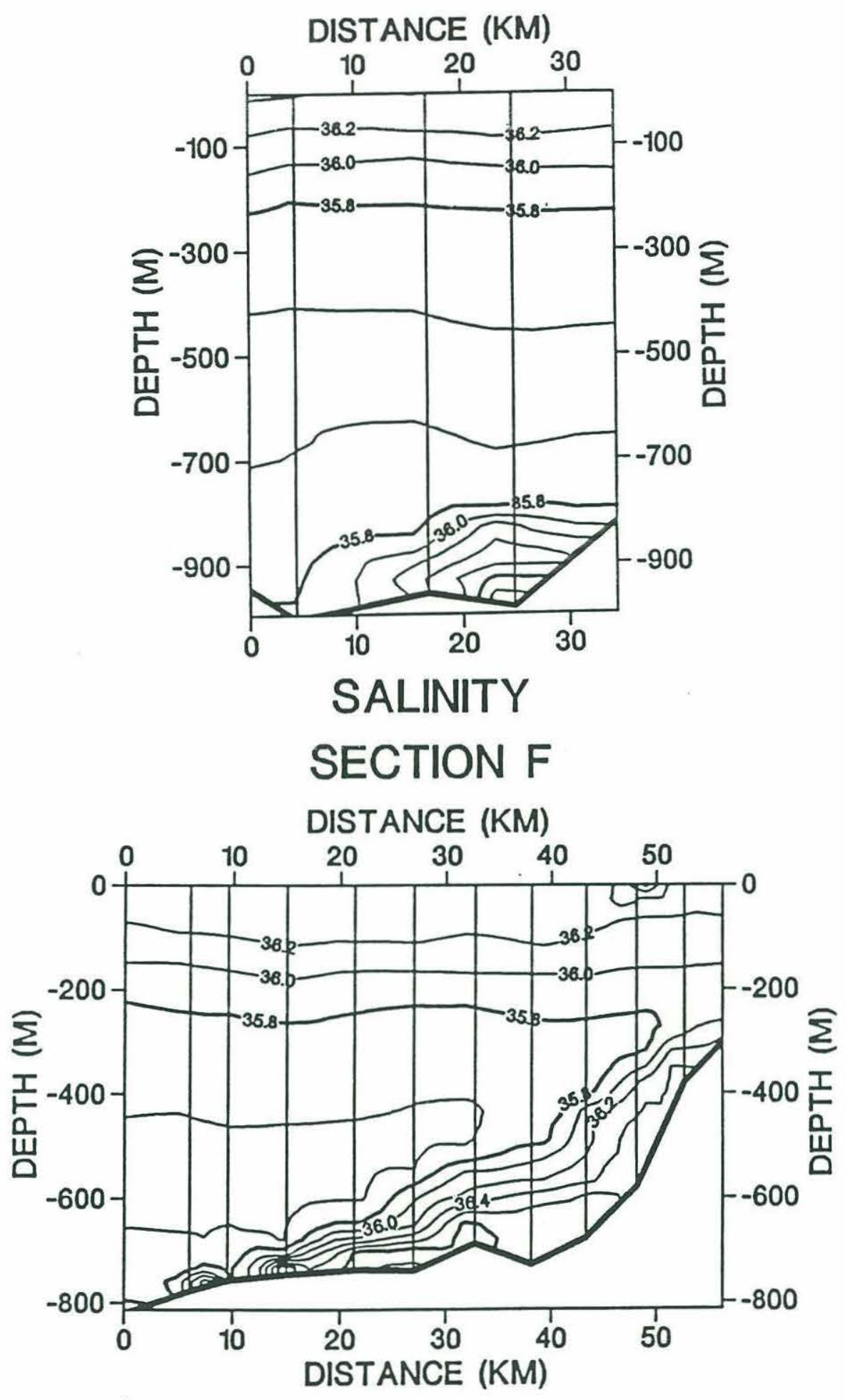

Figure 2.3: (d) Section F and FE 


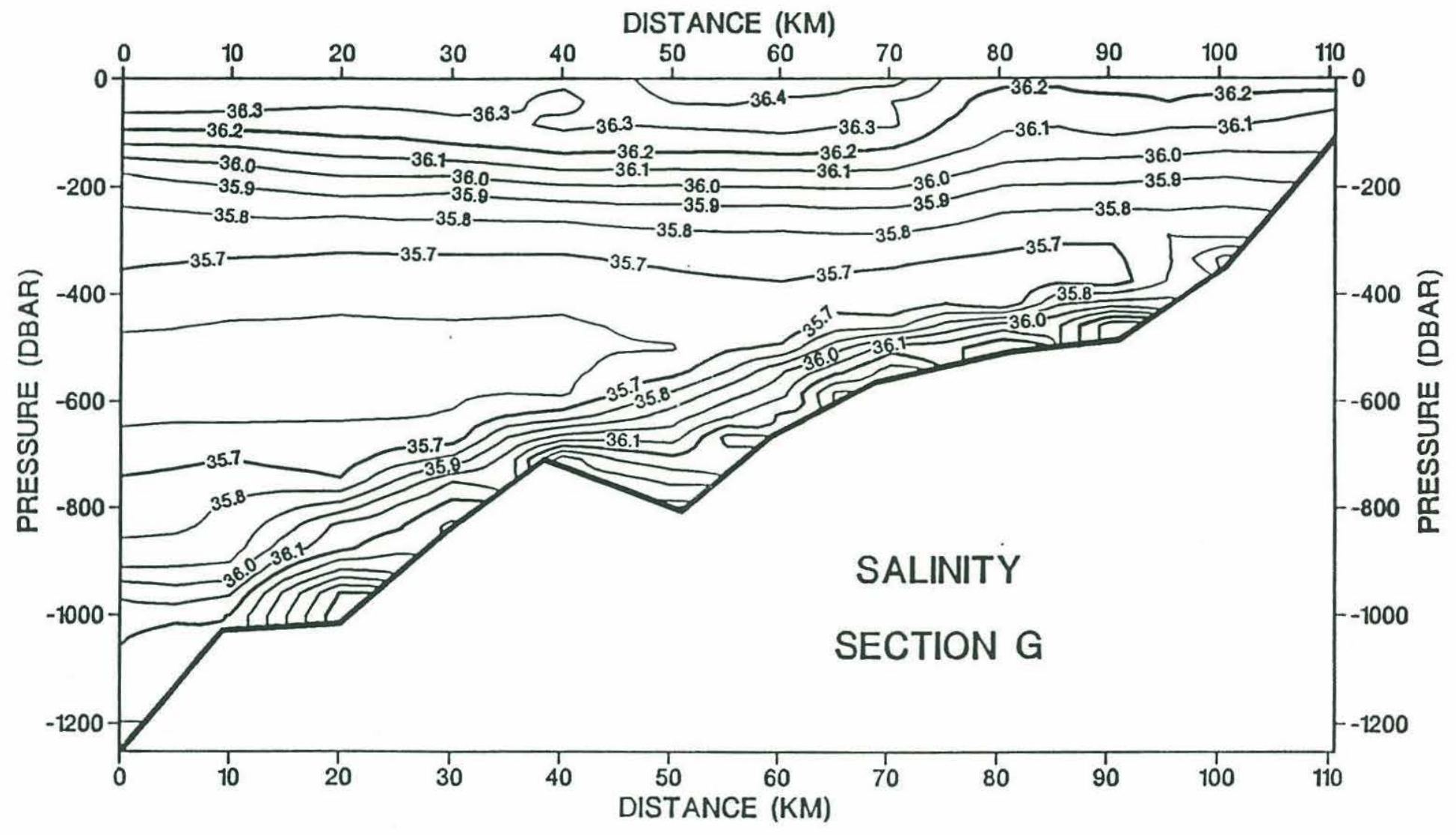

Figure 2.3: (e) Section G 


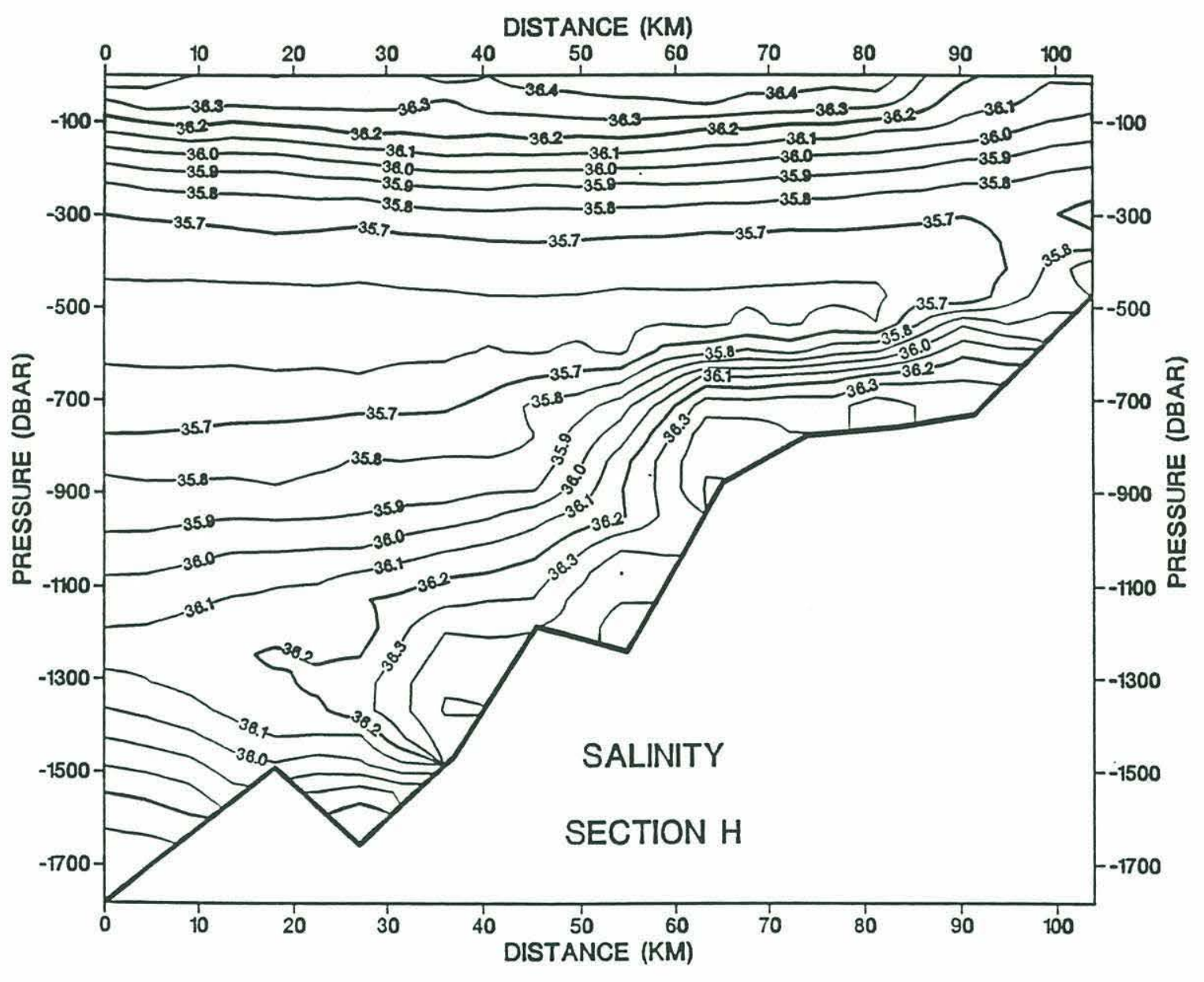

Figure 2.3: (f) Section $H$ 


\section{TEMPERATURE}

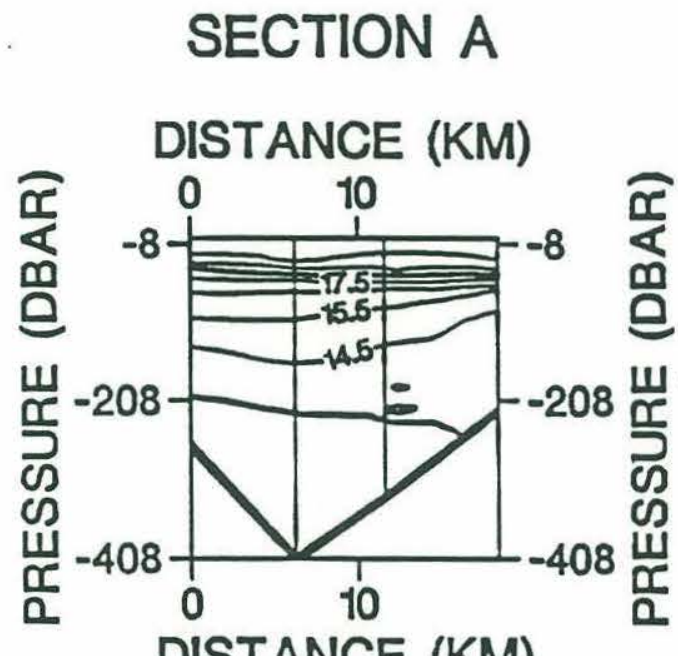

SECTION B

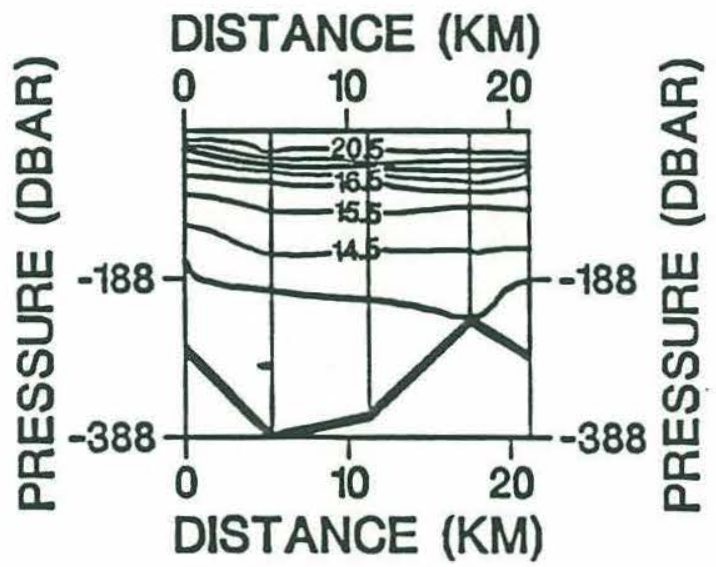

TEMPERATURE SECTION C DISTANCE (KM)

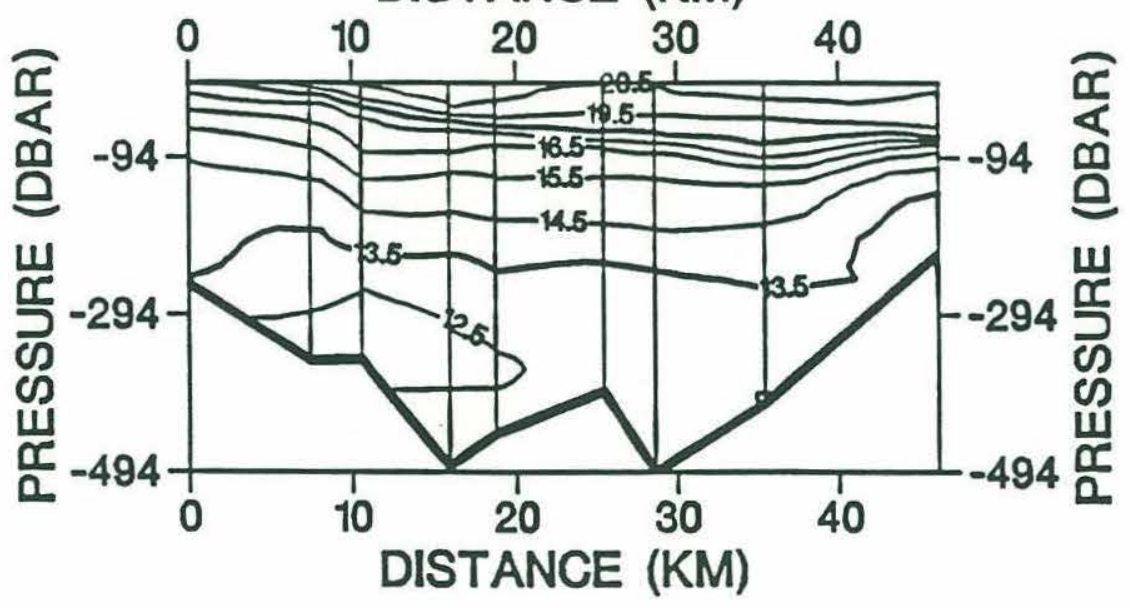

Figure 2.4: Temperature contours along distance versus depth section plots (in ${ }^{\circ} \mathrm{C}$ ). (a) Section A, B and C 

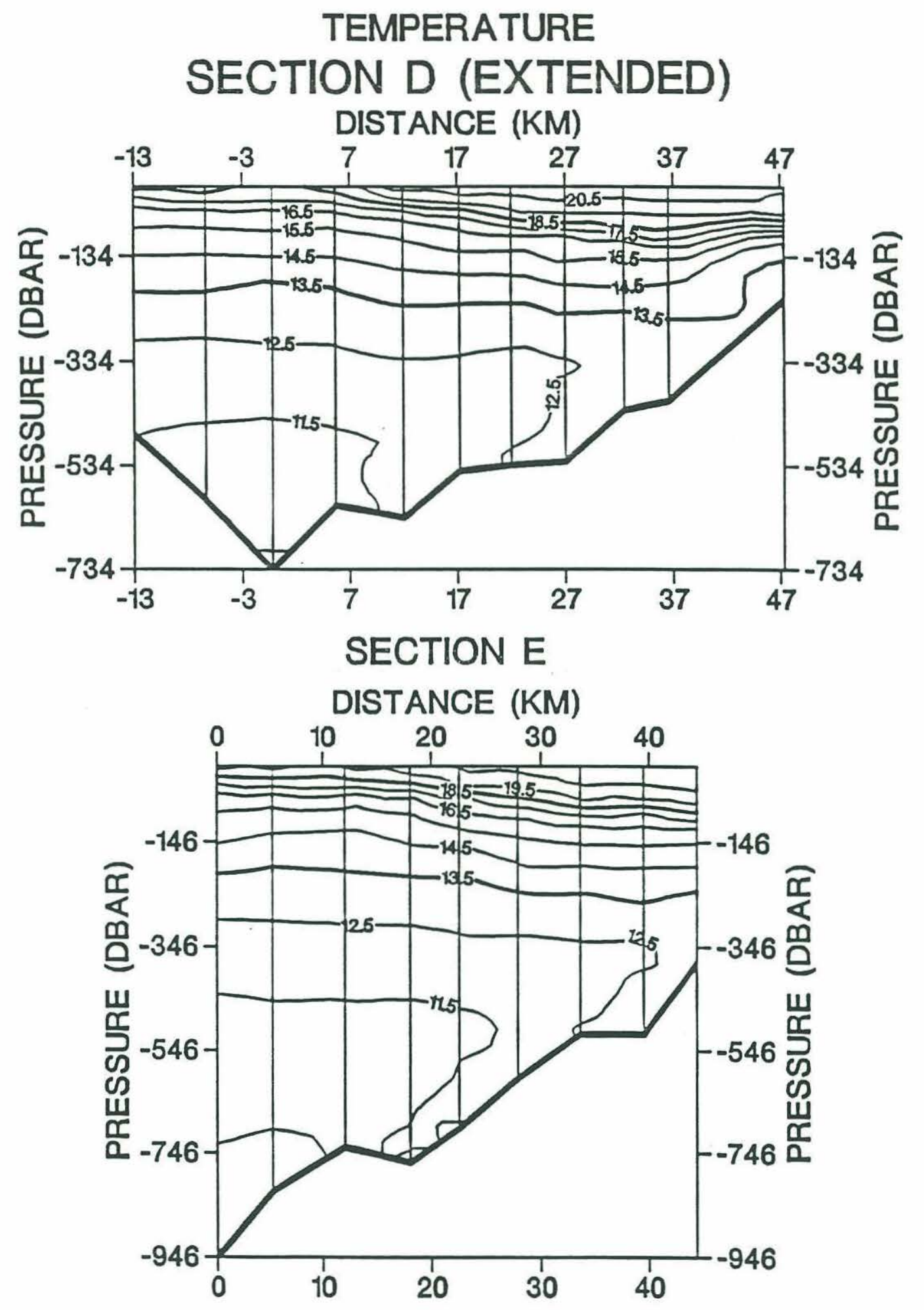

Figure 2.4: (c) Section D and E 


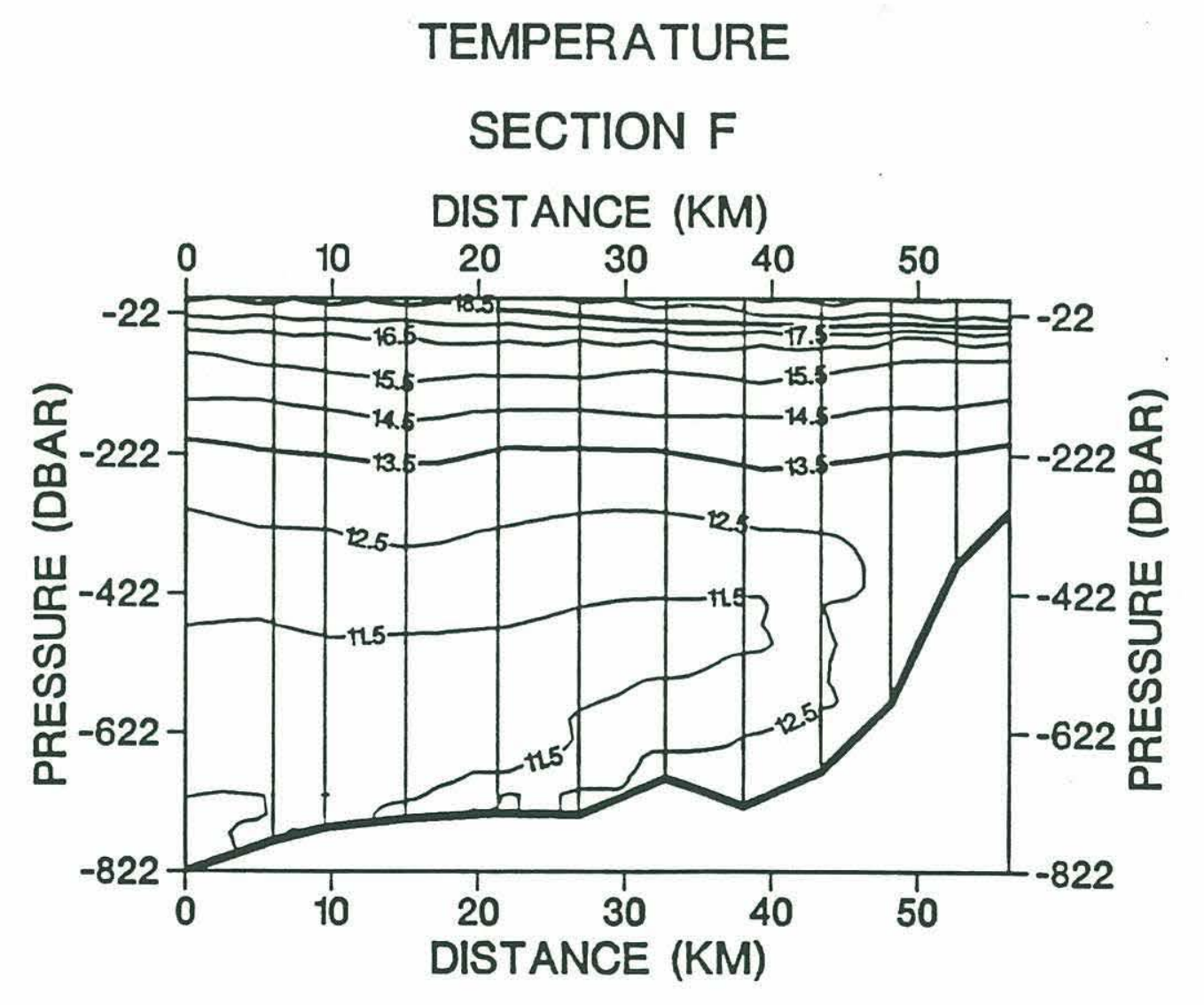

Figure 2.4: (d) Section F 


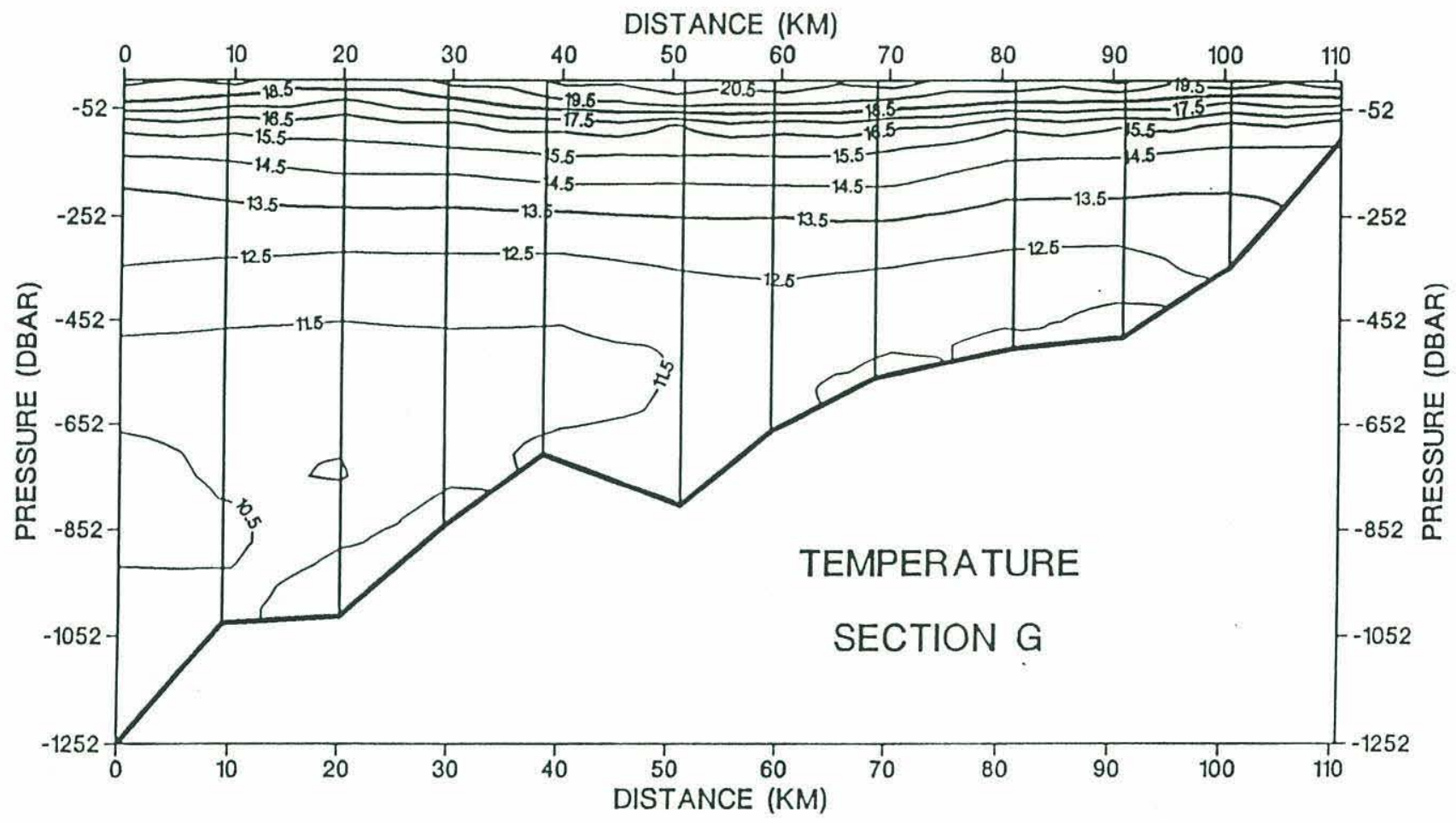

Figure 2.4: (e) Section G 


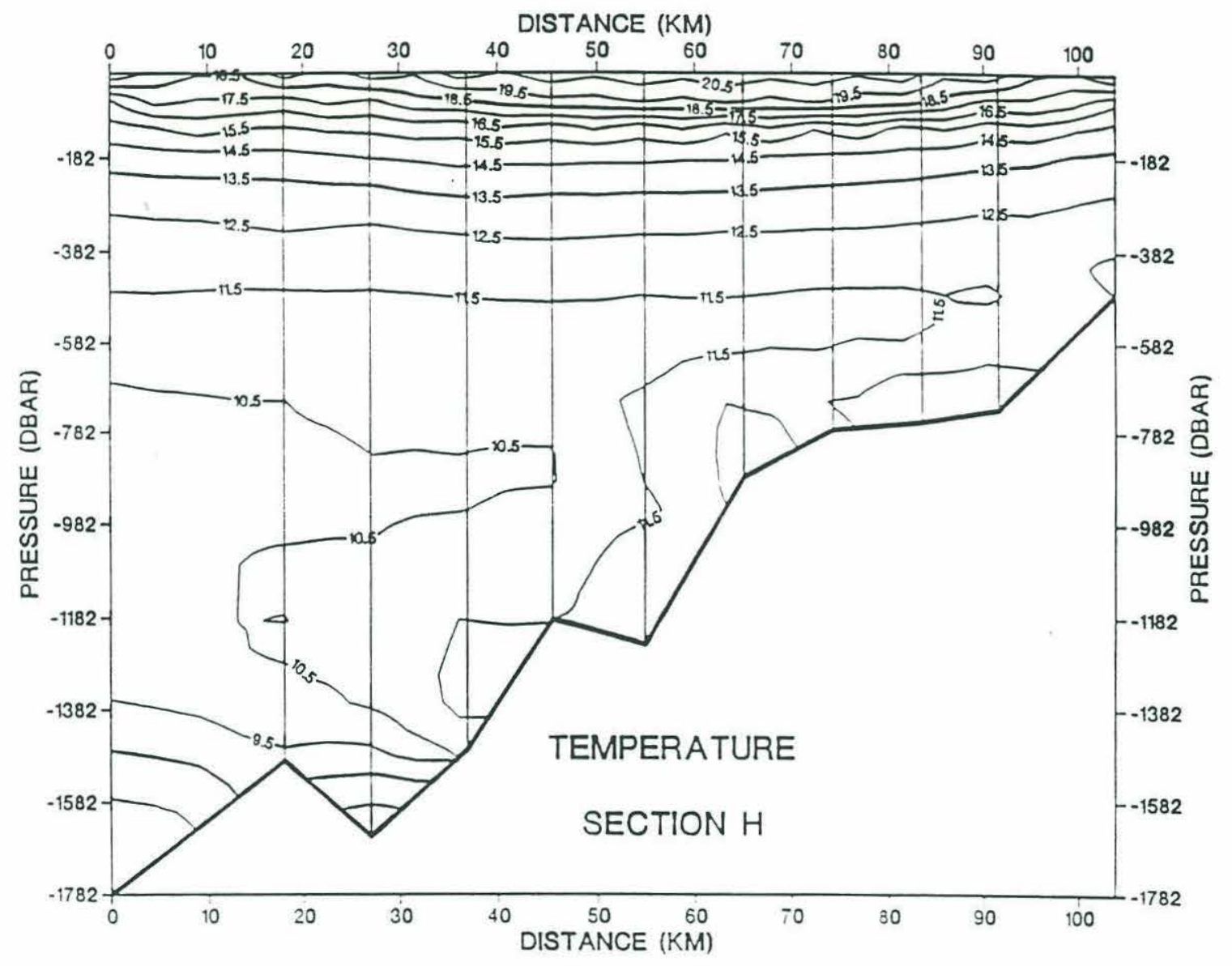

Figure 2.4: (f) Section $H$ 
SECTION A

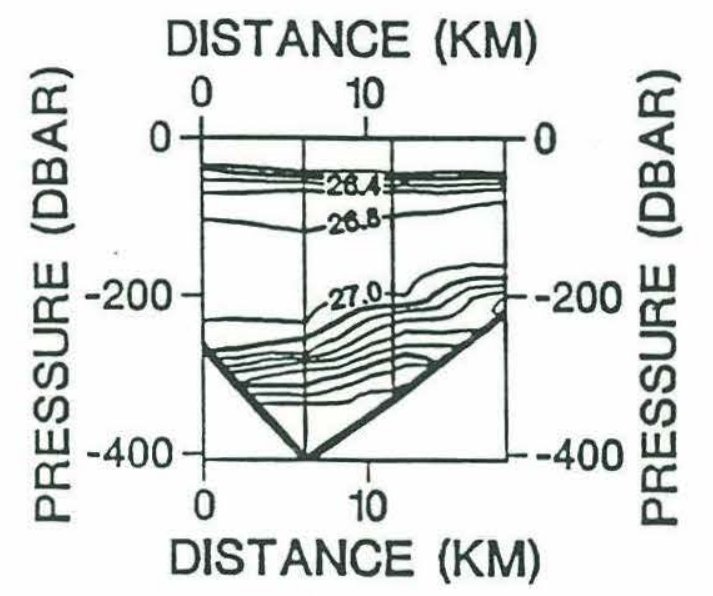

SECTION B

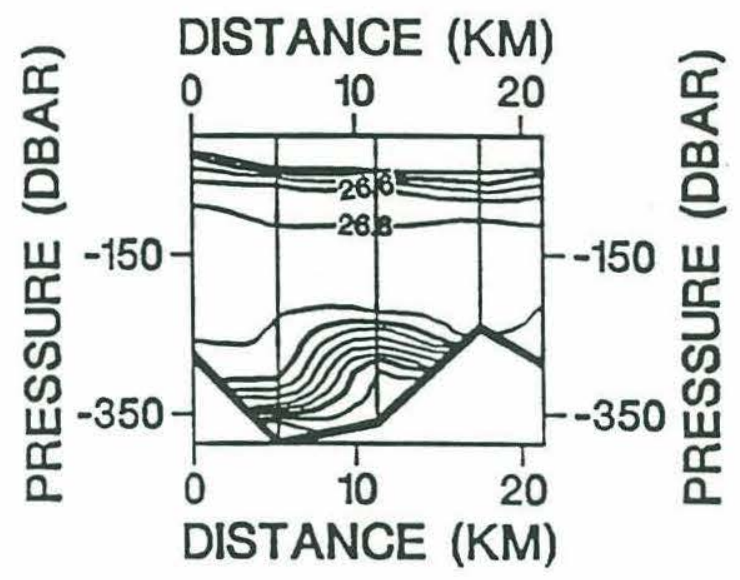

\section{SIGMA 0 SECTION C}

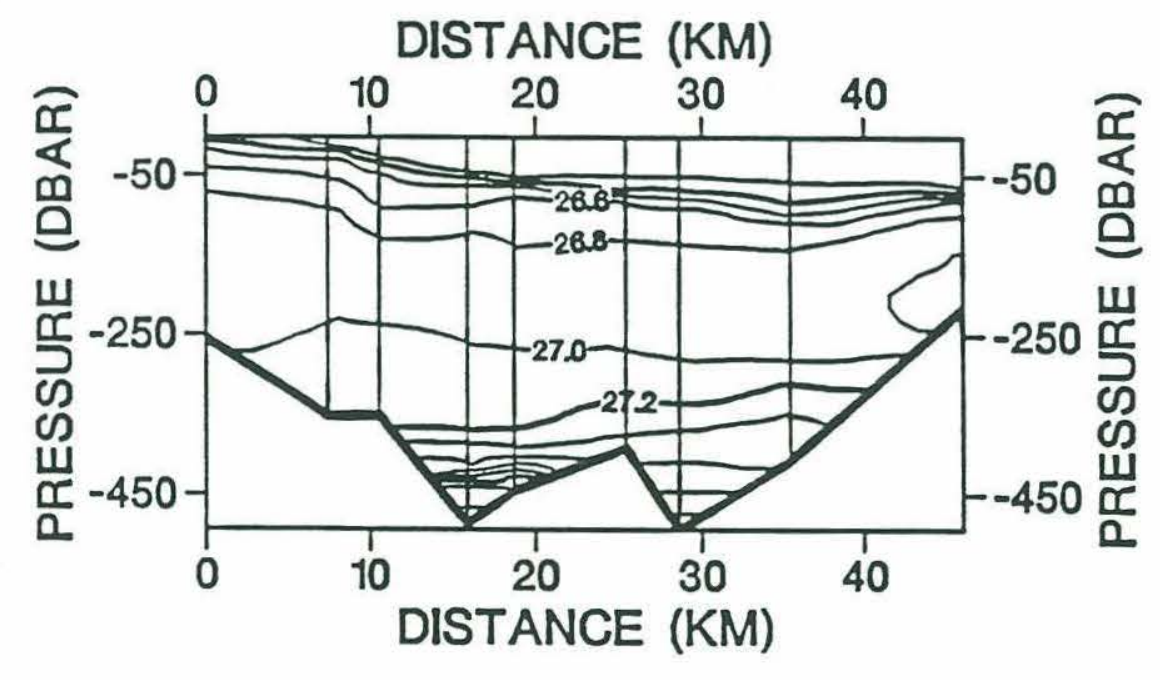

Figure 2.5: Potential density contours along distance versus depth section plots (in $\sigma)$. (a) Section A, B and C 


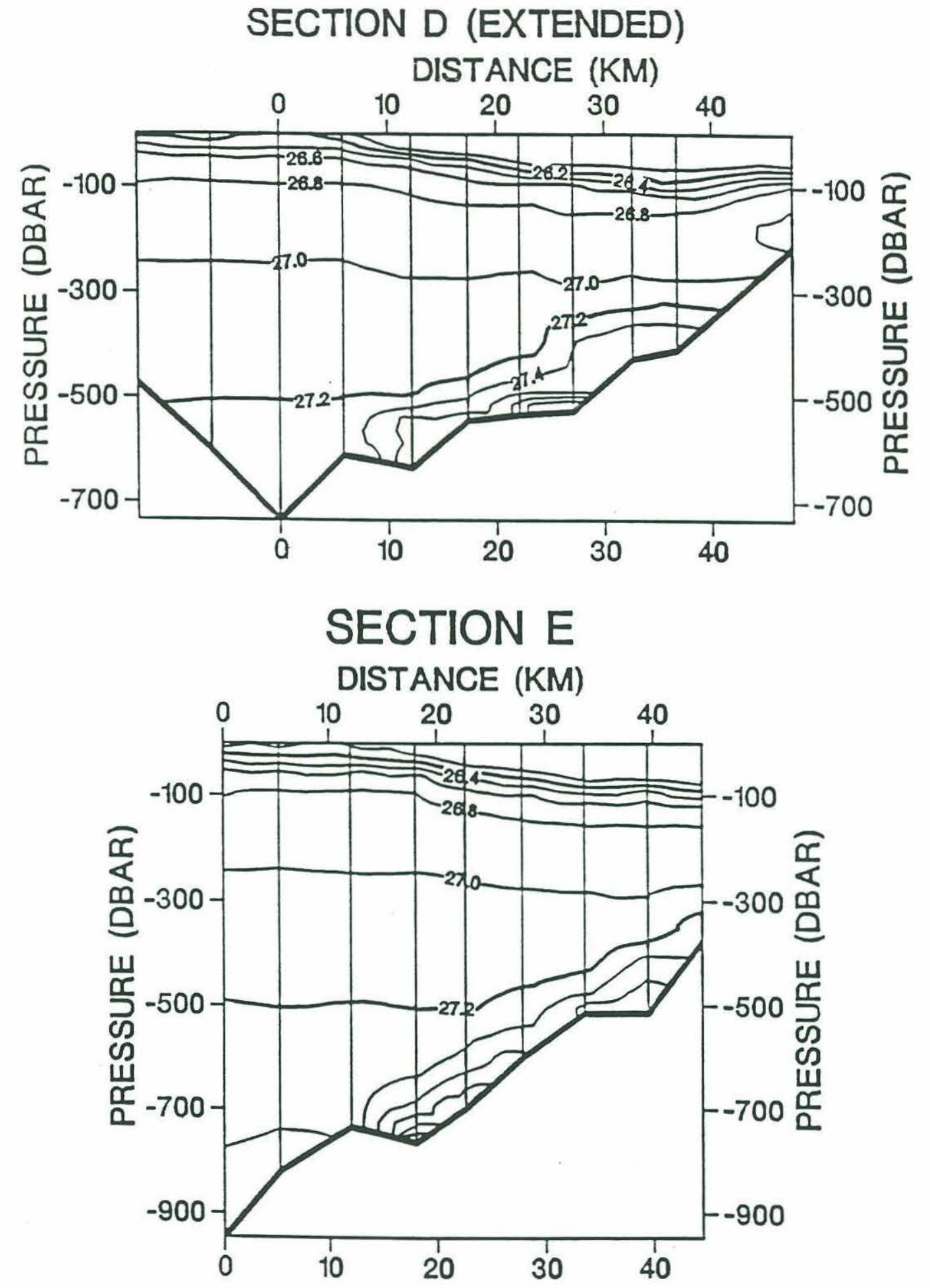

Figure 2.5: (c) Section D and E 


\section{SECTION FE}
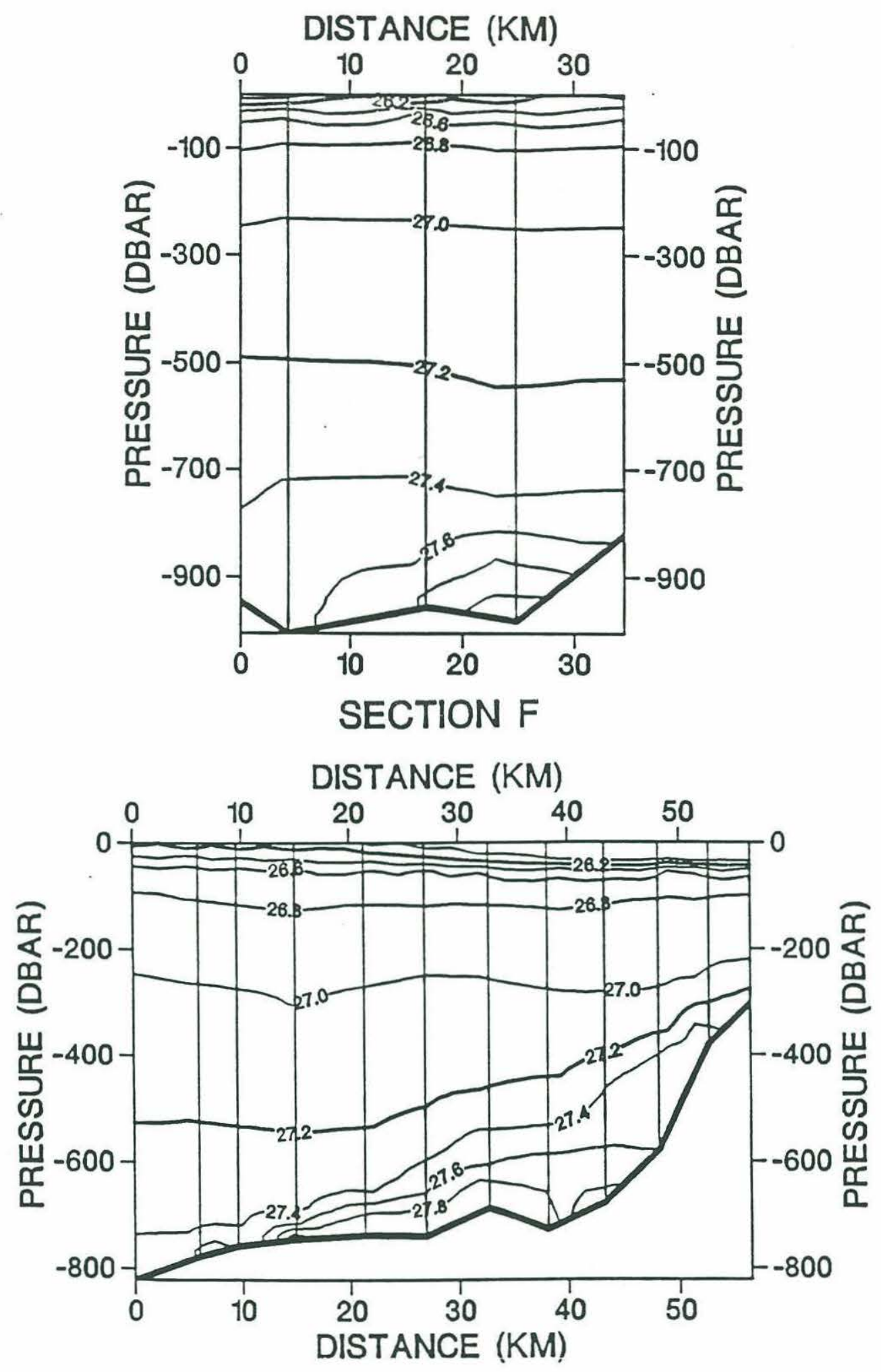

Figure 2.5: (d) Section F and FE 


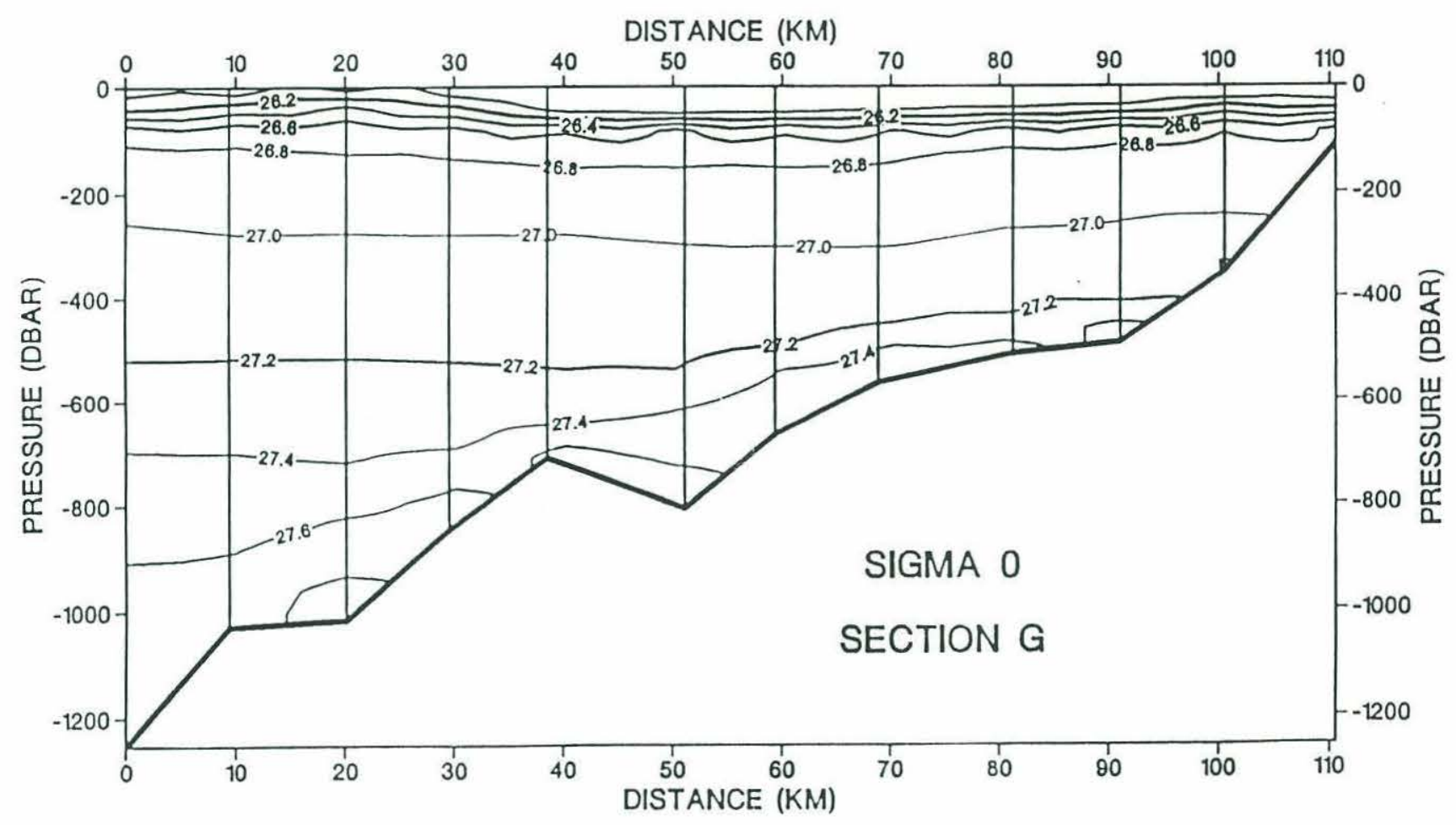

Figure 2.5: (e) Section G 


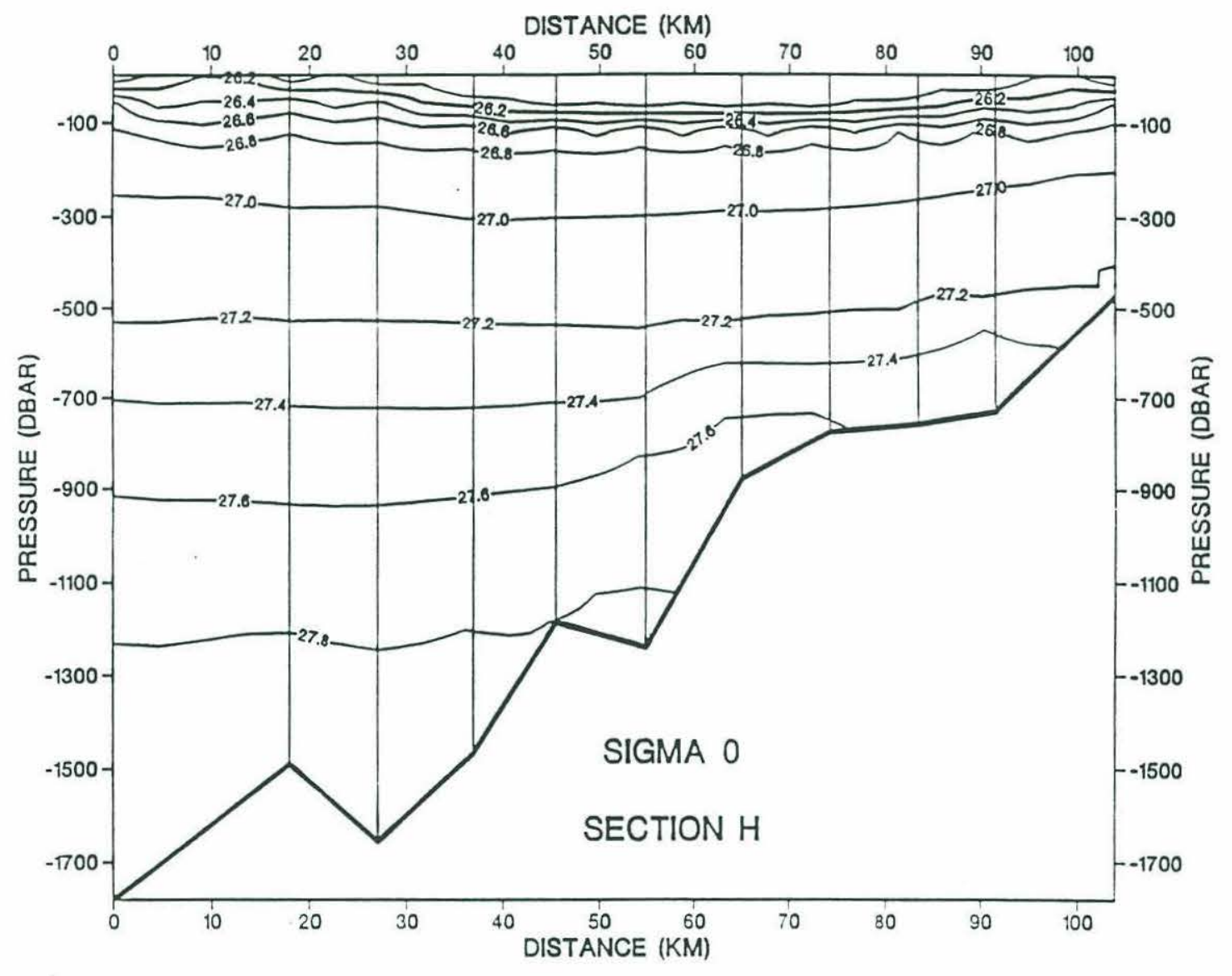

Figure 2.5: (f) Section $H$ 
A composite salinity section is contoured in figure 2.6(c). This figure follows the lower core of the Mediterranean outflow and includes section I through the strait and the stations with the maximum salinity for each of the sections A-H. The location of this 'core' salinity section is shown in figure 2.6(b). There are two prominent sills and one lesser sill along the strait: the Camarinal, the Spartel, and the Spartel West respectively. The 'pure' Mediterranean outflow water has salinity greater than 38.4 pss and can be seen as a large water mass to the East in this section. The large density contrast between this water and the relatively fresh Atlantic water forces a reverse estuarine circulation in which the Mediterranean water flows along the bottom to the west under the inflowing North Atlantic water. High salinity water is seen flowing over the Camarinal sill located at $0 \mathrm{~km}$ with a sill depth of $286 \mathrm{~m}$, near $5^{\circ} 45^{\prime} \mathrm{W}$. West of the Camarinal sill, the outflow spills over the secondary Spartel sill at $-25 \mathrm{~km}$ or $6^{\circ} \mathrm{W}$, with a slightly deeper sill depth of $316 \mathrm{~m}$. Our section $\mathrm{A}$ is located between the Spartel and Spartel West sills where some time dependent undulations of the diurnal tide may contaminate transport estimates. Section B is located near Spartel West, where current meters have shown less than a $20 \%$ variability to the mean current (Armi and Farmer(1988)).

As the Mediterranean water spills over the Spartel West sill it mixes with fresher North Atlantic water with $\mathrm{S}>36.0$ pss and moves down the continental slope as a bottom boundary current. Using the 36.4 isohaline to approximately define the top of the Mediterranean outflow resting on the bottom, figure 2.6(c) shows that the outflow maintains a fairly constant thickness of about $100 \mathrm{~m}$ from section $\mathrm{B}$ to section F, where the outflow begins to thicken. Within the strait however, the thickness of the Mediterranean outflow varies considerably.

At the Camarinal sill, two-layer hydraulic theory predicts the steady position of the layer interface between the Atlantic and Mediterranean water to be between 100-200 meters. Bryden, Brady and Pillsbury (1989) believe the demarcation of 

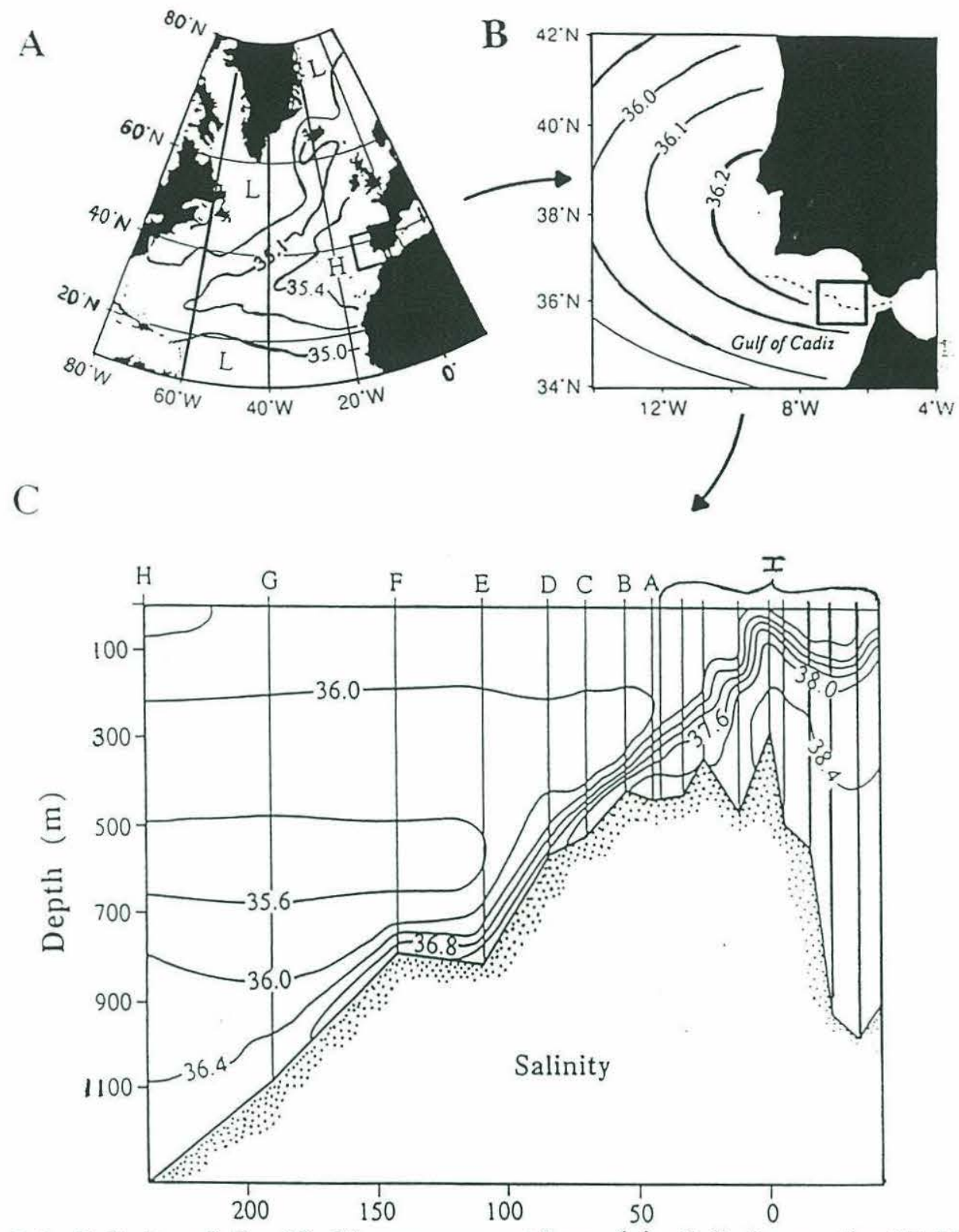

Figure 2.6: Salinity of the Mediterranean outflow. (a). Salinity on the 27.69 density surface in the North Atlantic (adapted from figure 2 of Reid (1979)). This density surface is $1200 \mathrm{~m}$ deep in the tropical North Atlantic and closely follows the subsurface salinity maximum characteristic of the Mediterranean outflow. (b). Salinity at 1200 $m$ depth in the eastern North Atlantic adapted from Maillard (1986). The dashed line shows the location of the composite section contoured in (c). (c). Composite section along the axis of the Mediterranean outflow. The composite section includes section I, through the Straits of Gibraltar, and one station from each section downstream. Each station downstream is the station at that section containing the saltiest outflow. When we discuss the spreading of the outflow, we show that the saltiest outflow is associated with the lower, deeper core of the Mediterranean outflow. 
outflowing Mediterranean water and inflowing Atlantic water is best represented by the 37.0 pss isohaline which has a zero mean along strait velocity as determined from current meters. They find this interface moves up and down in phase with the M2 tides about the mean position of $136 \mathrm{~m}$ with a standard deviation of $50 \mathrm{~m}$.

However, during the 1988 GofCExp, the 37.0 pss isohaline surfaced to only $18 \mathrm{~m}$ depth, well above either of these estimates, but still within the $95 \%$ confidence limits of Bryden et al (1989). ${ }^{1}$ Section I, then, should not be interpreted as a mean picture because the currents within the strait are so highly variable. Bryden et al. also found that the surfacing of the 37 pss isohaline correlated with enhanced tidal transport to the west (see also Armi and Farmer 1988). Therefore, the Gulf of Cadiz Expedition probably ${ }^{2}$ occupied the Camarinal sill station during the westward phase of the tide.

The eight sections (A through $\mathrm{H}$ ), oriented perpendicular to the outflow all show strongly sloping isohalines with the saline outflow next to the bottom (see figure 2.3). Note that these sections are perpendicular to the historical axis of the flow and sloping isopycnals represent flow east/west through the section assuming the down-stream velocity is approximately geostrophic unlike in the cross-steam velocity. The temperature of the outflow at the sill is $12.9^{\circ} \mathrm{C}$ and the stratified inflow ranges in temperature from 13 to $20^{\circ} \mathrm{C}$ (see section I in figure 2.4(a)). The outflow is relatively uniform in temperature initially and thus the salinity contours are good indicators of the isopycnal structure. Figure 2.5 shows the potential density structure of the sections. Isopycnals greater than $27.6 \sigma_{\theta}$ intersect the bottom topography to the north and south of the outflow in sections A-F (see also figure 2.7(d)). Thus, from the strait until past section $\mathrm{F}$ the outflow is a bottom trapped density current. After this initial descent, the temperature becomes increasingly non uniform with the

\footnotetext{
${ }^{1}$ The interface is also tilted across the strait, but since we only have measurements at the center of the strait, we can not assess the cross stream structure of the interface.

${ }^{2}$ find out
} 
warmest water appearing in the north and colder water to the south (eg. section F has the $12.5^{\circ} \mathrm{C}$ contour separating the northern and southern part of the outflow). In general, tilts of isohalines visually correspond well to isopycnal tilts. Similarly, potential density referenced to $1000 \mathrm{~m}$ is visually indistinguishable from potential density contours referenced to the surface. Therefore, we use the salinity sections through most of this discussion.

Sections A and B, near the strait, sampled the overflow about $45 \mathrm{~km}$ after it passes over the Camarinal sill and where the outflow is still confined to a channel only $15 \mathrm{~km}$ wide and is not allowed to spread freely (figures 2.3(b) and 2.5(b)). Further west at Section C (figure 2.3(b)) the outflow has descended into the Gulf of Cadiz and accelerates to a maximum speed of $1.3 \mathrm{~m} / \mathrm{s}$. The flow turns right as it is deflected by the Coriolis force and continues around the northern continental slope of the Gulf of Cadiz slowly crossing $f / h$ contours.

The maximum salinity of the outflow at section D has decreased to 37.6 pss which indicates that substantial mixing has already taken place within $80 \mathrm{~km}$ from the sill. At sections D and E, two regions of high salinity are separated by a ridge in the topography (near $30 \mathrm{~km}$ in figure 2.3(c)). Historically the water associated with these relative maxima have been called the two cores (Ambar and Howe (1979a). The historical studies of this region have emphasized the development and downstream path of these two cores. The two core development and a detailed description of the spreading of the outflow will be discussed further in Section 2.4.

Further downstream, between sections $\mathrm{E}$ and $\mathrm{F}$ some of the outflow is deflected southward presumably channeled by the submarine canyon near $35^{\circ} 58^{\prime} \mathrm{N}, 7^{\circ} 10^{\prime} \mathrm{N}$ (figure 1.7, figure 2.3(d)). The high salinity outflow is banked up against the left side of a ridge in Section FE. Note that section FE is the offshore connection between sections $\mathrm{E}$ and $\mathrm{F}$ and tries to capture the leakage described in Madelain (1970). The salinity of the outflow at section $\mathrm{FE}$ is 37.1 while the maximum salinity at section 

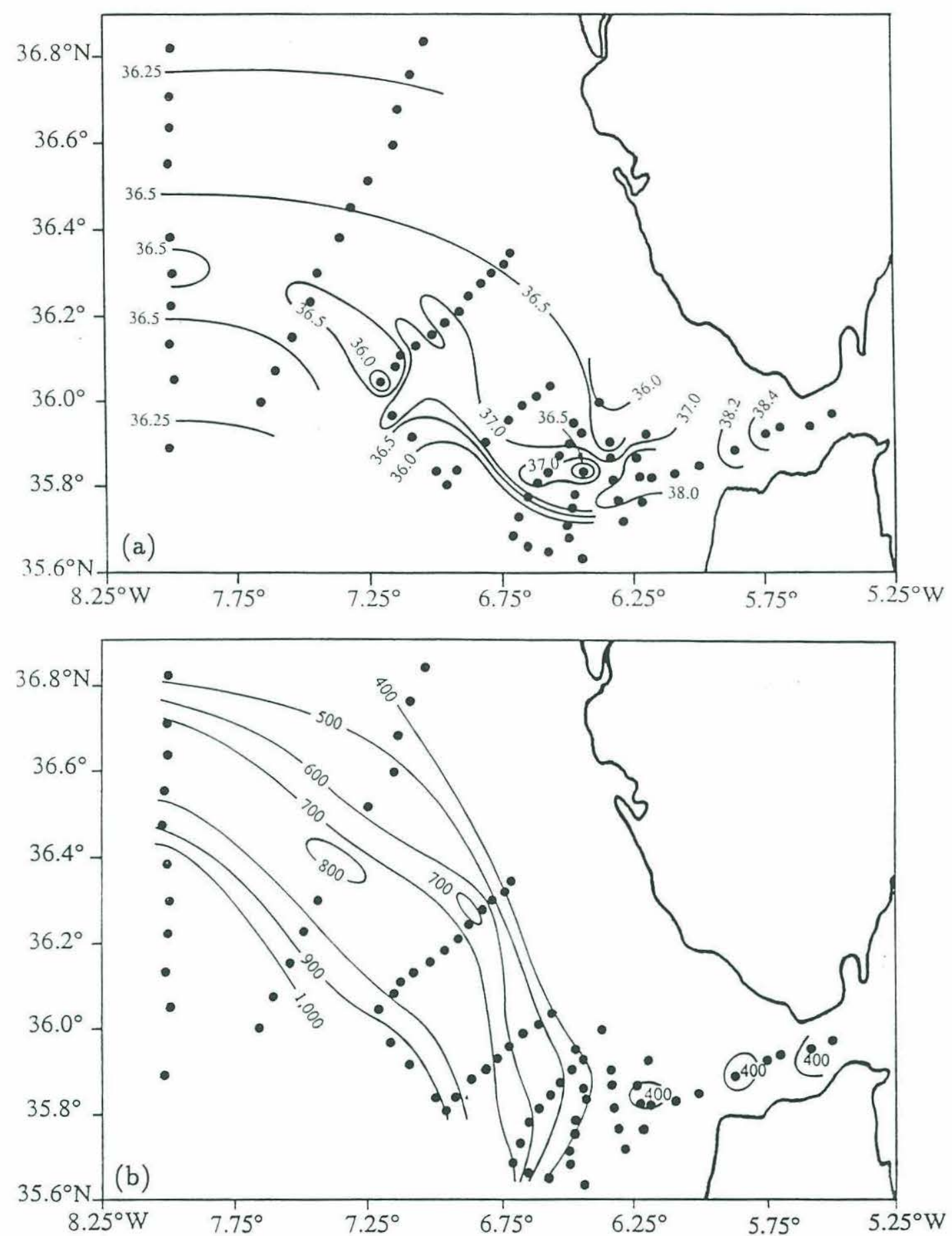

Figure 2.7: Properties at the depth of maximum salinity at each station in the Gulf of Cadiz. (a) the maximum salinity. (b) the pressure at the maximum salinity. Where no outflow is present the bottom salinity, etc is plotted. 

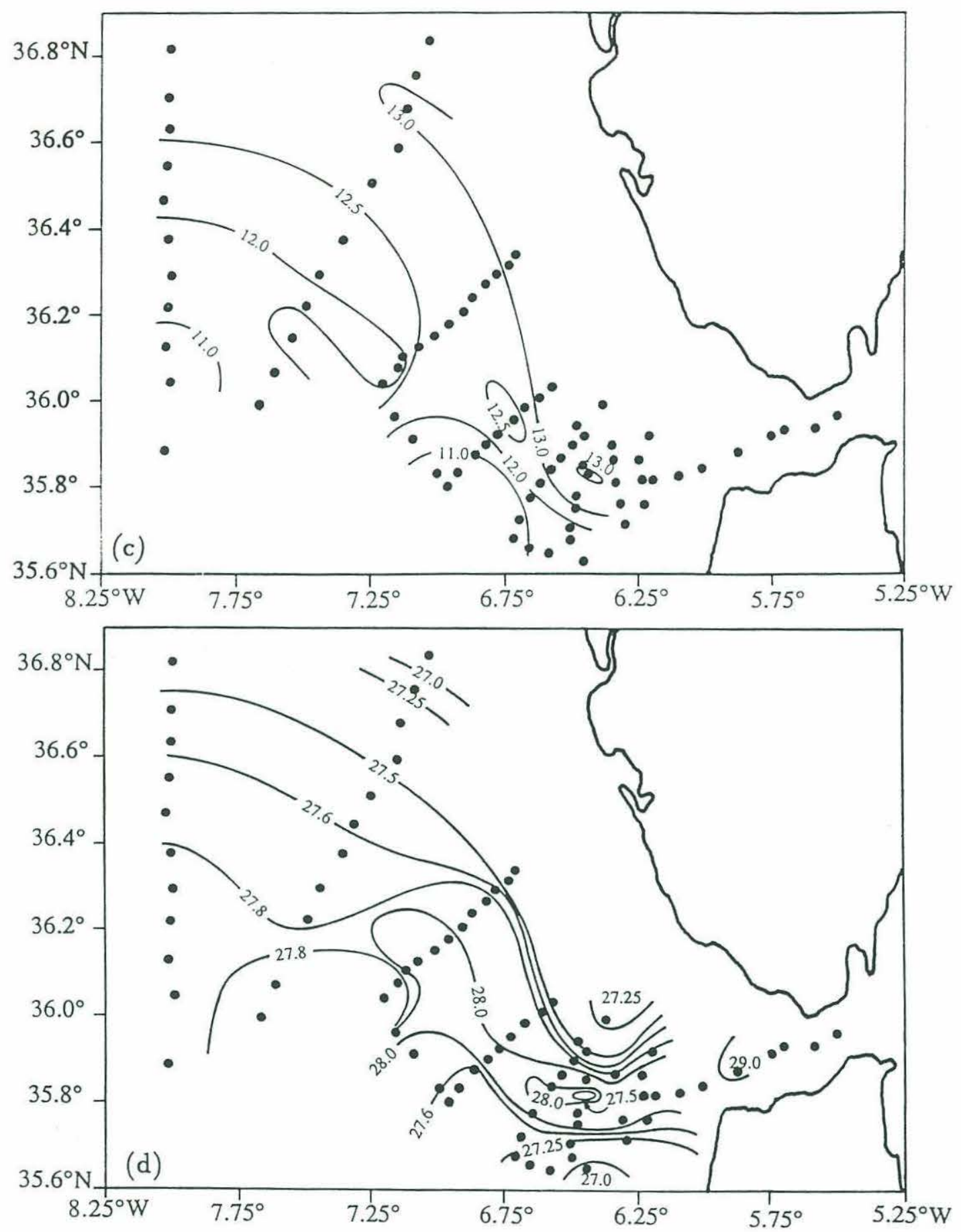

Figure 2.7: (c) the potential temperature at the maximum salinity. (d) potential density at the maximum salinity referenced to the surface. 
$\mathrm{E}$ is 37.5 . The high salinity of this vein of southward moving outflow indicates that it must have originated from the lower, saltier core at section E. Section $\mathrm{F}$ has an even higher maximum salinity (37.2) than section FE. Therefore, either the saltiest water has not been redirected southward by the submarine canyon or more intense mixing has taken place along the path from section $\mathrm{E}$ to section $\mathrm{FE}$ than along the path from section $\mathrm{E}$ to section $\mathrm{F}$. Approaching section $\mathrm{F}$, the ridge separating the two cores disappears and the northern, shallower core experiences an abrupt local steepening of the topography (see also figure 2.7(b)). The salinity section at section F (figure 2.3(d)) does not indicate a separation of the outflow into two salinity cores. However, the geostrophic velocity section and the XCP section both do contain two cores of high velocity separated by a velocity minimum (near $35 \mathrm{~km}$ in figure 2.8(c) and $2.9(\mathrm{c}))$.

At section $\mathrm{G}$ the outflow is spread to its maximum width of over $90 \mathrm{~km}$ before it encounters the steep slopes off Cape St. Vincent. At section $\mathrm{H}$ where the steep slope is first encountered the outflow narrows. The maximum salinity value of the outflow begins to lift off the bottom (figure 2.3(e)). Below the Mediterranean outflow, at section $\mathrm{H}$ where the outflow separates from the bottom, there is an eastward circulation in the geostrophic velocities (figure 2.9(e)) with countercurrent speeds exceeding $10 \mathrm{~cm} / \mathrm{sec}$. This eastward circulation, also referred to as the sub-Mediterranean undercurrent, was first reported by Ambar (1979b) between $9^{\circ} \mathrm{W}$ and $8^{\circ} \mathrm{W} 30^{\prime} \mathrm{W}$ with speeds from $6-22 \mathrm{~cm} / \mathrm{sec}$ at $1400 \mathrm{~m}$.

South of the main outflow near $0 \mathrm{~km}$ at section $\mathrm{H}$, there is a mid depth salinity maximum near 1250 dbar. This is the diffuse Mediterranean 'tongue' that extends into the North Atlantic. The focus of this study is on the energetic portion of the outflow where it remains in contact with the bottom. Transport estimates presented here do not include this diffuse tongue (ie. the most offshore station pair at section $\mathrm{G}$ ). Also, when analyzing the momentum budget of the current, the ambient stratification 


\section{VELOCITY}
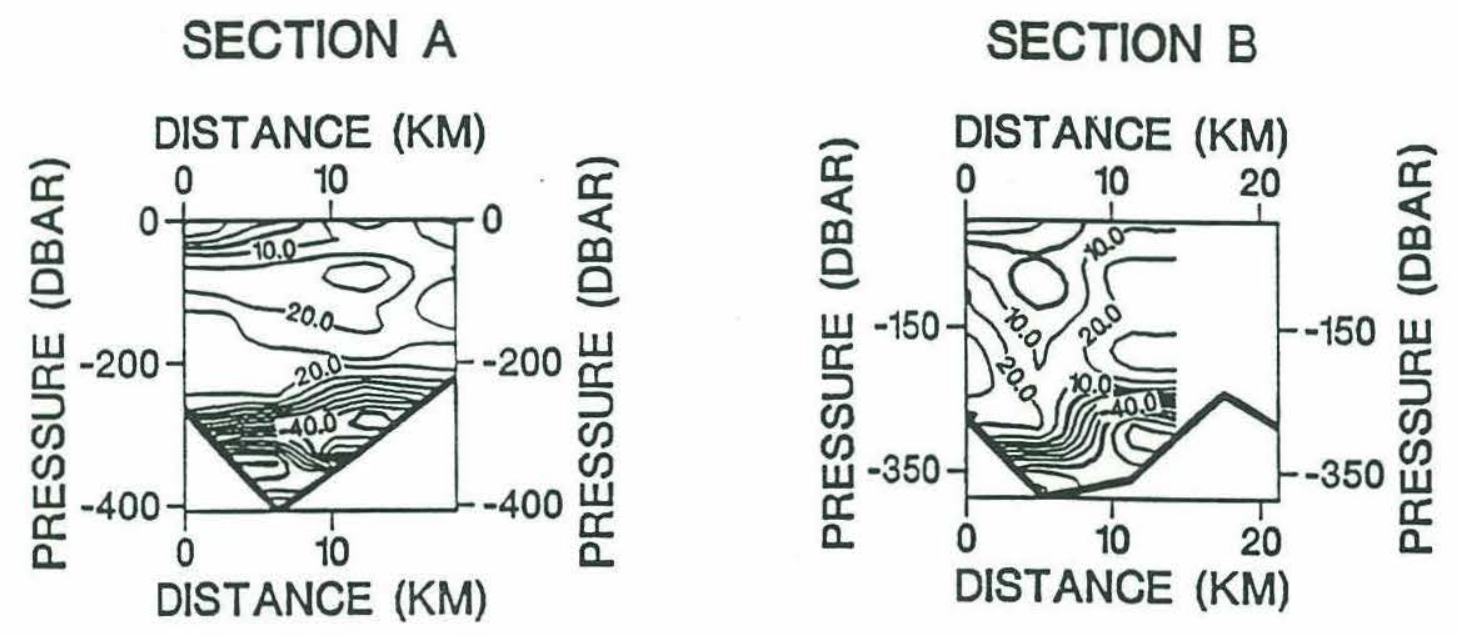

\section{SECTION C}

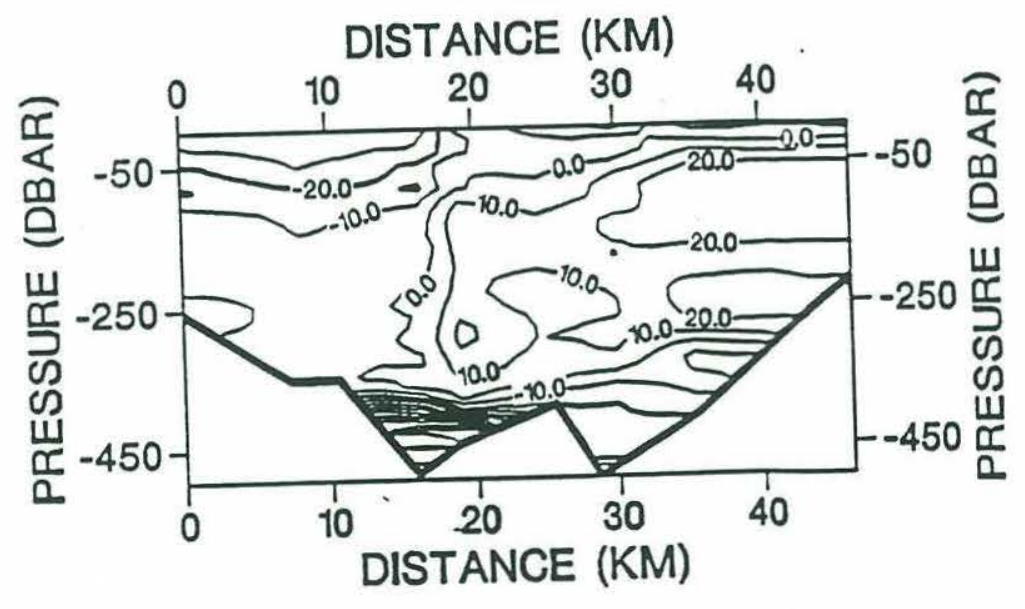

Figure 2.8: XCP velocity contours along distance versus depth section plots (in $\mathrm{cm} / \mathrm{sec}$ ). (a) Section A, B and C 

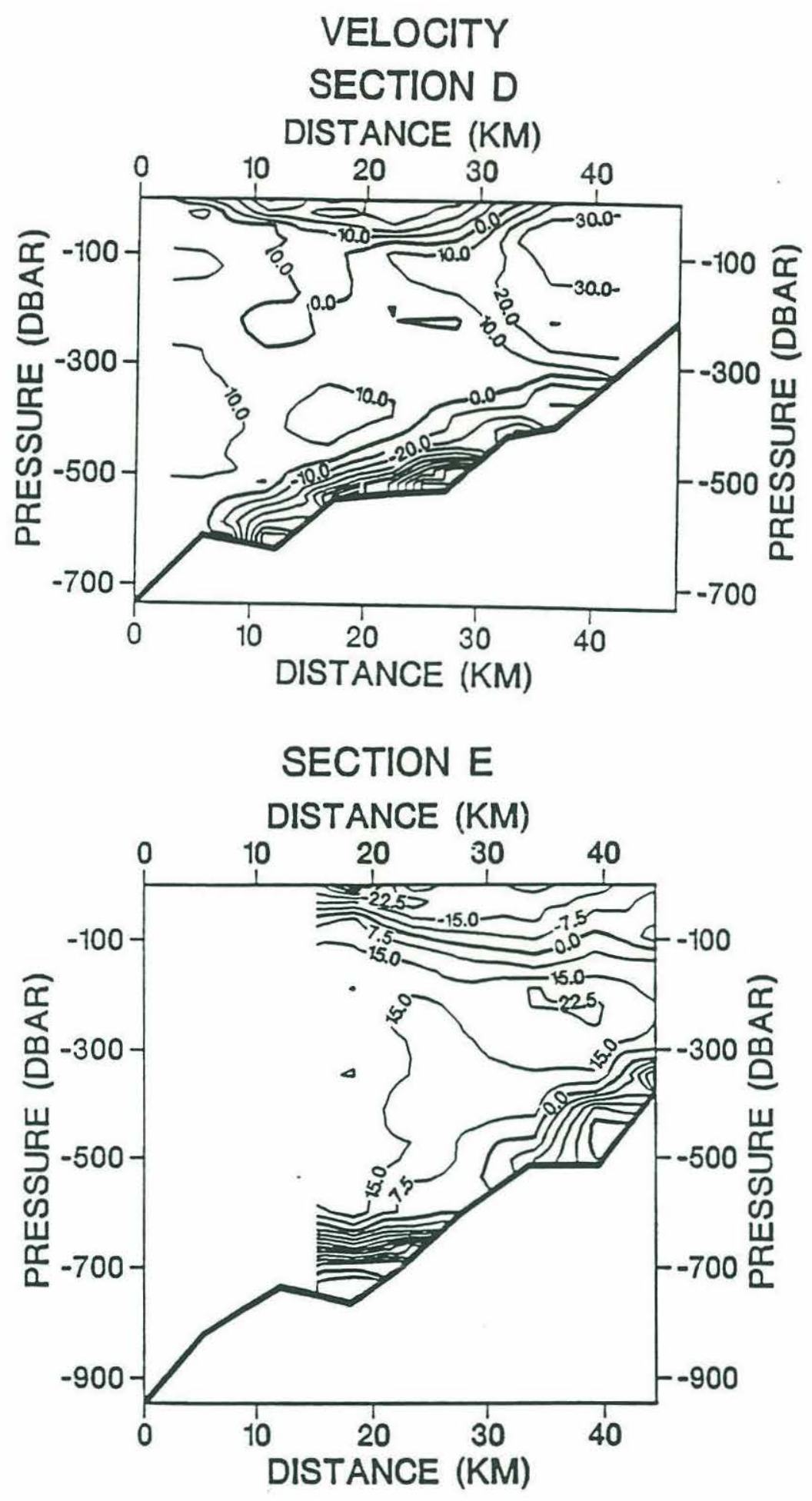

Figure 2.8: (c) Section D and E 


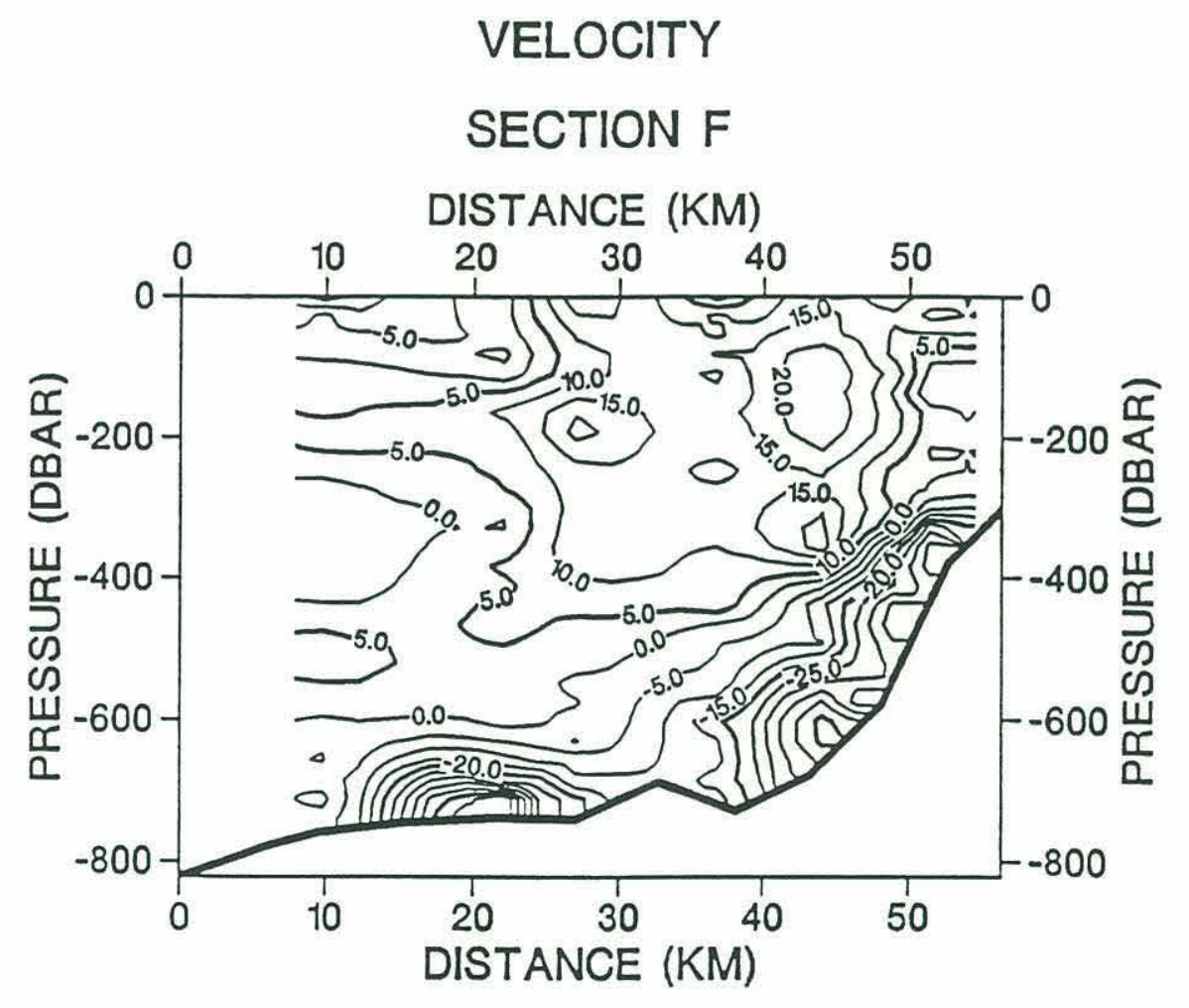

Figure 2.8: (d) Section F 


\section{VELOCITY}

\section{SECTION A}

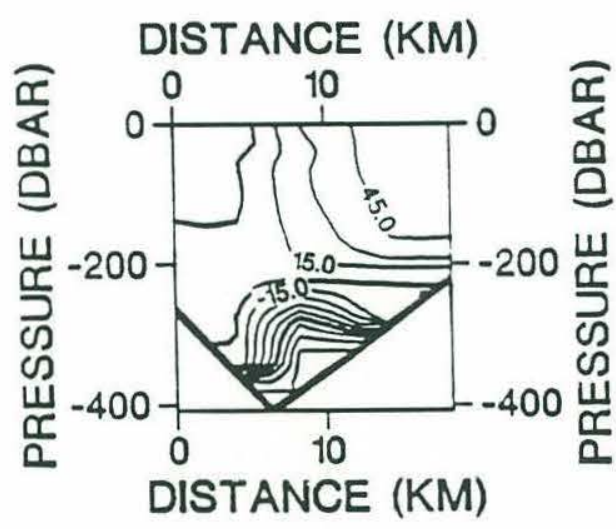

SECTION B

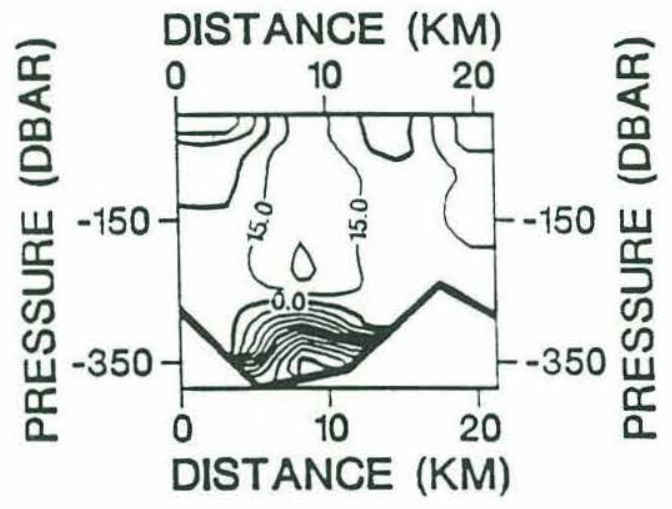

\section{SECTION C}

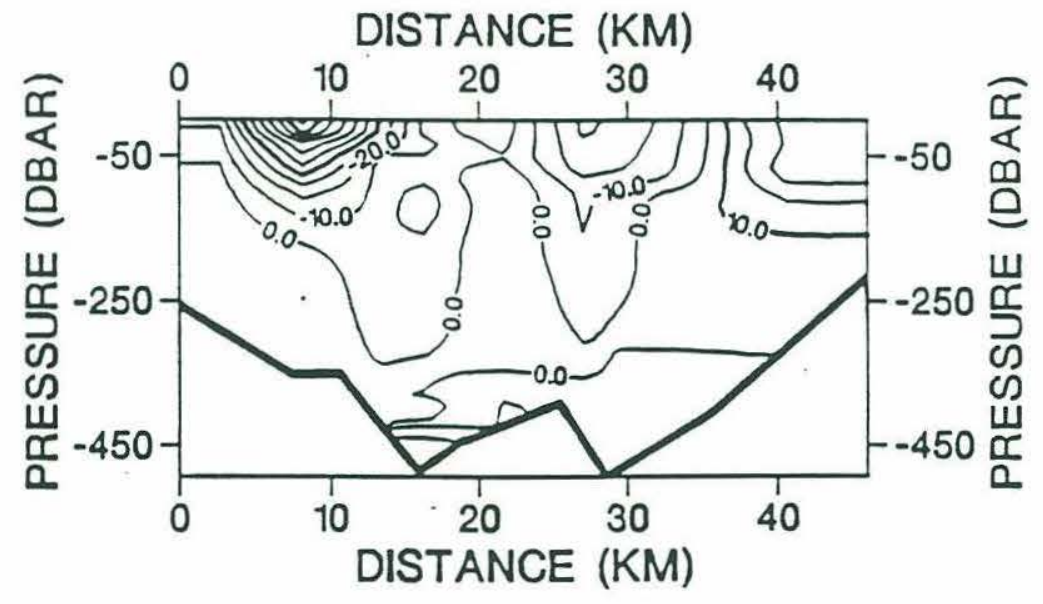

Figure 2.9: Geostrophic velocity contours along distance versus depth section plots (in $\mathrm{cm} / \mathrm{sec}$ ). (a) Section $\mathrm{A}, \mathrm{B}$ and $\mathrm{C}$ 

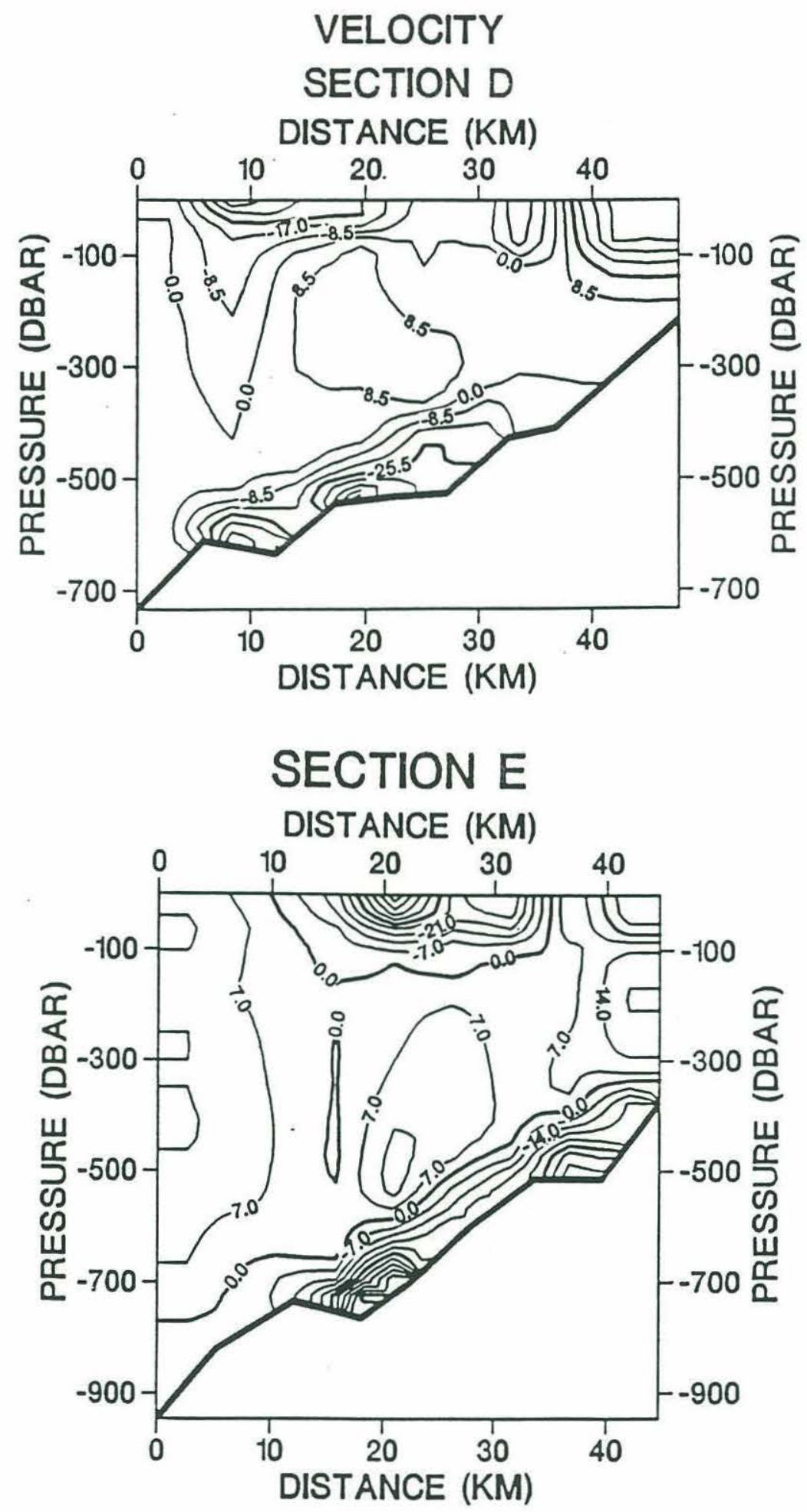

Figure 2.9: (c) Section D and E 


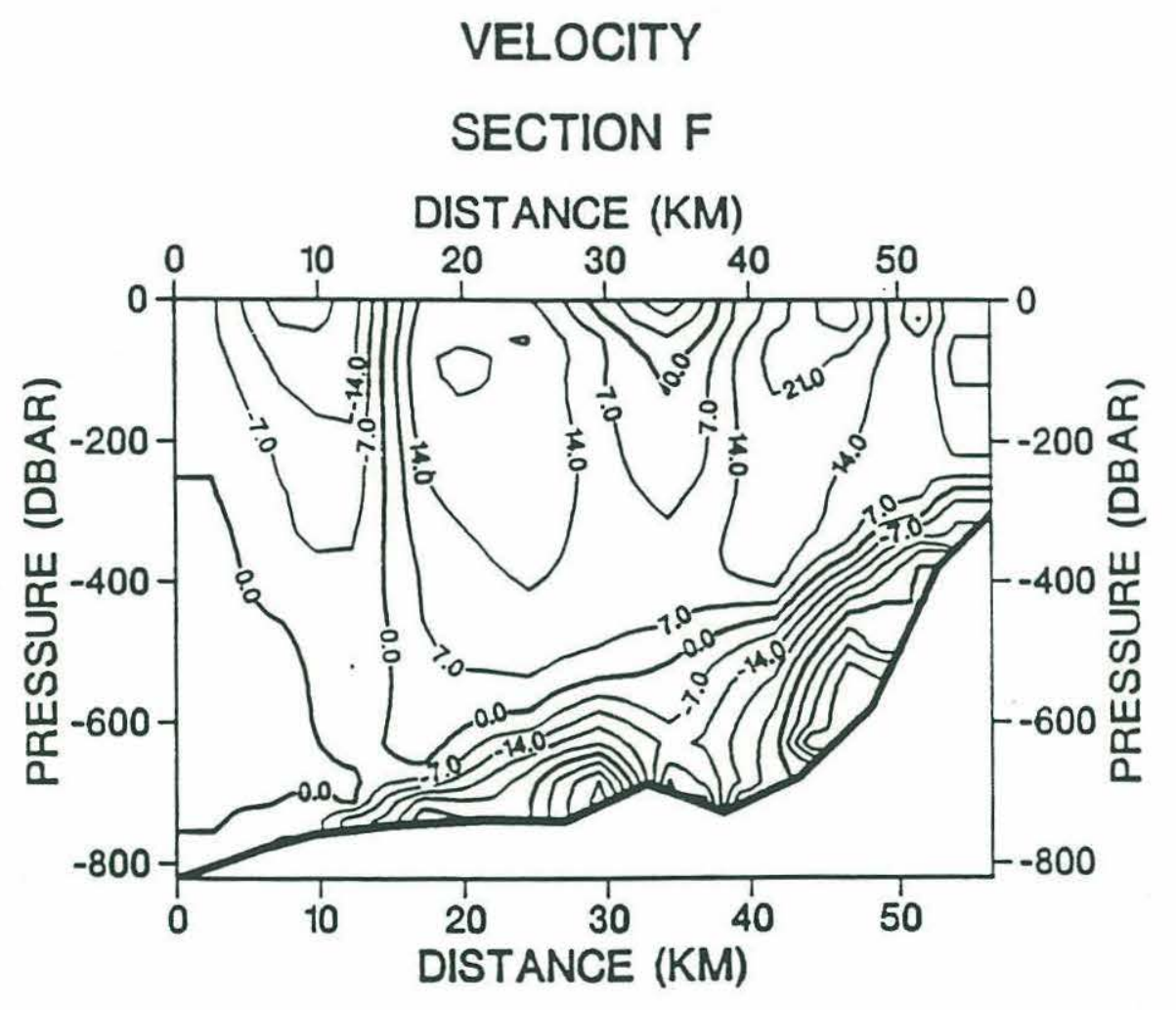

Figure 2.9: (d) Section F 


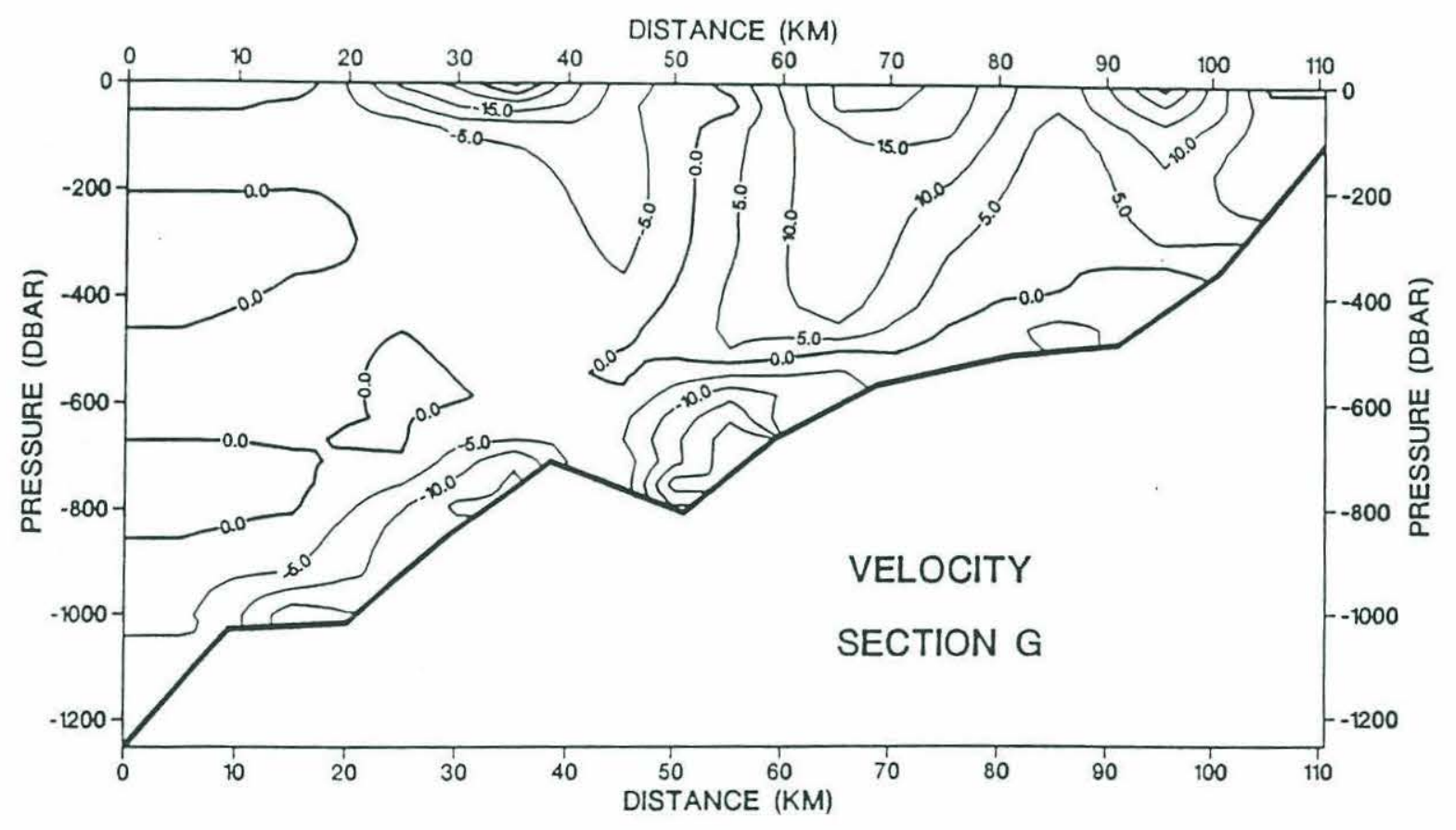

Figure 2.9: (e) Section G 


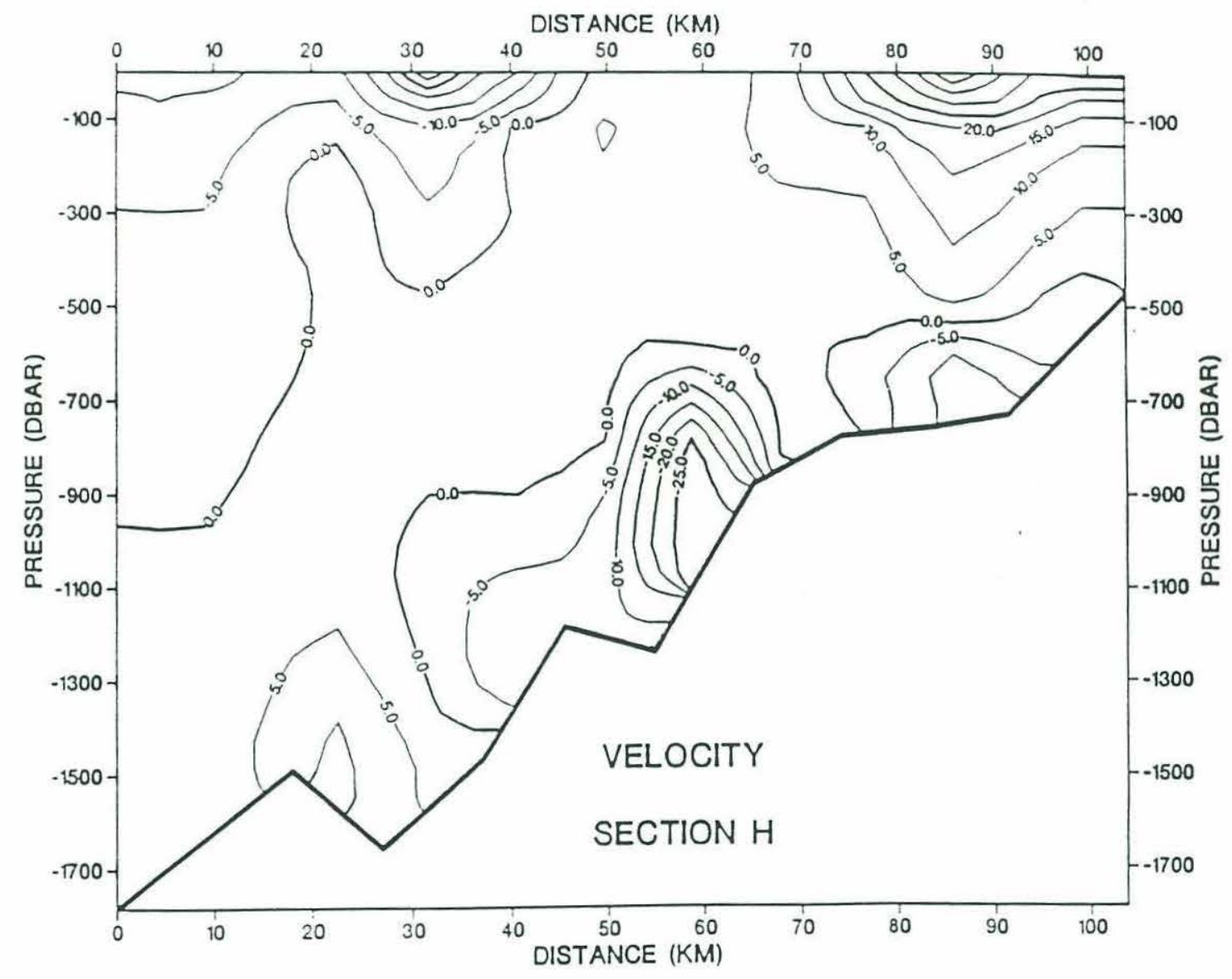

Figure 2.9: (f) Section $H$ 
is assumed to be set up over much longer time scales than the advection time scale or transit time of a particle through the Gulf of Cadiz (10 days).

Historical estimates of the flow through the strait find a transport of Mediterranean water of about $1 \mathrm{~Sv}$ which is slightly larger than the transport of $0.9 \mathrm{~Sv}$ we found at Section A, $45 \mathrm{~km}$ further downstream of the Camarinal sill. The transport nearly doubles just $40 \mathrm{~km}$ further downstream at section $\mathrm{E}$ indicating intense mixing coincident with the rapid erosion of the maximum salinity. At sections A and B the maximum flow speed is about $75-110 \mathrm{~cm} / \mathrm{s}$. The maximum velocity increases slightly to $130 \mathrm{~cm} / \mathrm{s}$ at section $\mathrm{C}$. By the time the flow reaches section $\mathrm{F}$, the $\mathrm{XCP}$ velocities have decreased to less than $60 \mathrm{~cm} / \mathrm{s}$, generally about $30 \mathrm{~cm} / \mathrm{s}$.

The outflow changes its salinity from 38.4 to 36.1 within the $100 \mathrm{~km}$ sampled from our sections A-F. The Mediterranean water has already been modified enough by mixing with the fresh North Atlantic water that it cannot form deep water in the open North Atlantic and will become an interflow at mid-depths. To examine the intense mixing process necessary to modify the flow so quickly, we need to consider the evolution of higher order properties like salt flux and momentum. The absolute flow field must be determined. In section 2.3 the method we used to obtain reference velocities is described and compared to the historical salinity minimum reference level.

\subsection{Determination of the absolute velocity}

Determining the absolute velocity field from geostrophic velocities requires the specification of a velocity reference, a level of known motion. The XCP is designed to measure changes in potential induced when water, which conducts electricity, passes through the earth's magnetic field. Because it measures changes relative to a baseline, only relative velocities are measured. Therefore, as with velocities estimated from the 
thermal wind relation, the XCP gives only the velocity shear and a reference velocity must be given to determine the absolute flow field.

In this study we assume that 1) water with low salinity or temperature has no source in the Mediterranean and thus must be motionless or moving towards the Mediterranean and 2) that the Mediterranean does not gain or lose salt so the salt flux across a section must vanish. A two step referencing scheme was employed; first, the XCPs were adjusted so that the along stream velocity at the base of the Atlantic layer is zero, ${ }^{3}$ then, the integrated salt flux across each section was calculated and a single reference velocity for each section was determined such that salt flux across each section was conserved. In appendix 2.3 these two steps will be described in detail. We compare the resulting flow field with Ochoa and Bray (1991) data where a similar salt flux constraint was imposed. The resulting level of no motion is just below the temperature minimum and varies in depth and $\sigma_{\theta}$ across the section. We find that this referencing scheme produces a more consistent picture of the outflow than using other reference levels, especially the traditional salinity minimum.

\footnotetext{
A).

${ }^{3}$ The base of the Atlantic layer is defined as the depth of the temperature minimum (see appendix
} 


\subsection{Horizontal structure}

A prominent feature of the Mediterranean outflow is its rapid spreading. In this section we will examine the horizontal structure of the outflow through examination of the salinity field and the XCP velocity field. The evolution of two cores of high salinity will be described and then a close look at the velocity field will demonstrate that the horizontal spreading of the outflow plume on the continental slope in the Gulf of Cadiz contains both a barotropic and baroclinic component.

\section{The width}

From each section A-H (figure2.3) we can see the spreading of the outflow along the continental slope. The outflow is confined to a channel from section A and B and its width is fixed in this channel to approximately $12 \mathrm{~km}$. Downstream at section C the flow is still contained in a channel that has doubled in width to $24 \mathrm{~km}$. Notice that the shallowest isobath the current follows at section C is the $409 \mathrm{~m}$ isobath (figure 2.3(a). The ridge, near $25 \mathrm{~km}$ in figure 2.3(a), has a depth of $390 \mathrm{~m}$ while the deepest station along this section reaches a depth of $494 \mathrm{~m}$. The average height of the outflow at this section is approximately $90 \mathrm{~m}$, therefore these undulations in bottom topography, although small relative to open ocean and general circulation standards, are significant for outflow.

Further downstream of section $\mathrm{C}$ the flow is free to spread out upon the continental slope. We define the width of the flow to be the maximum separation to the north and south of the 36.3 isohaline along each section. ${ }^{5}$ The width of the outflow for each section is given in table 2.1. The outflow increases its width by nearly an order of magnitude where it reaches its maximum extent at Section G. Downstream

\footnotetext{
${ }^{5}$ Note that the edges of the flow are usually quite distinct. See for example figure A.1 which shows two stations right next to each other.
} 


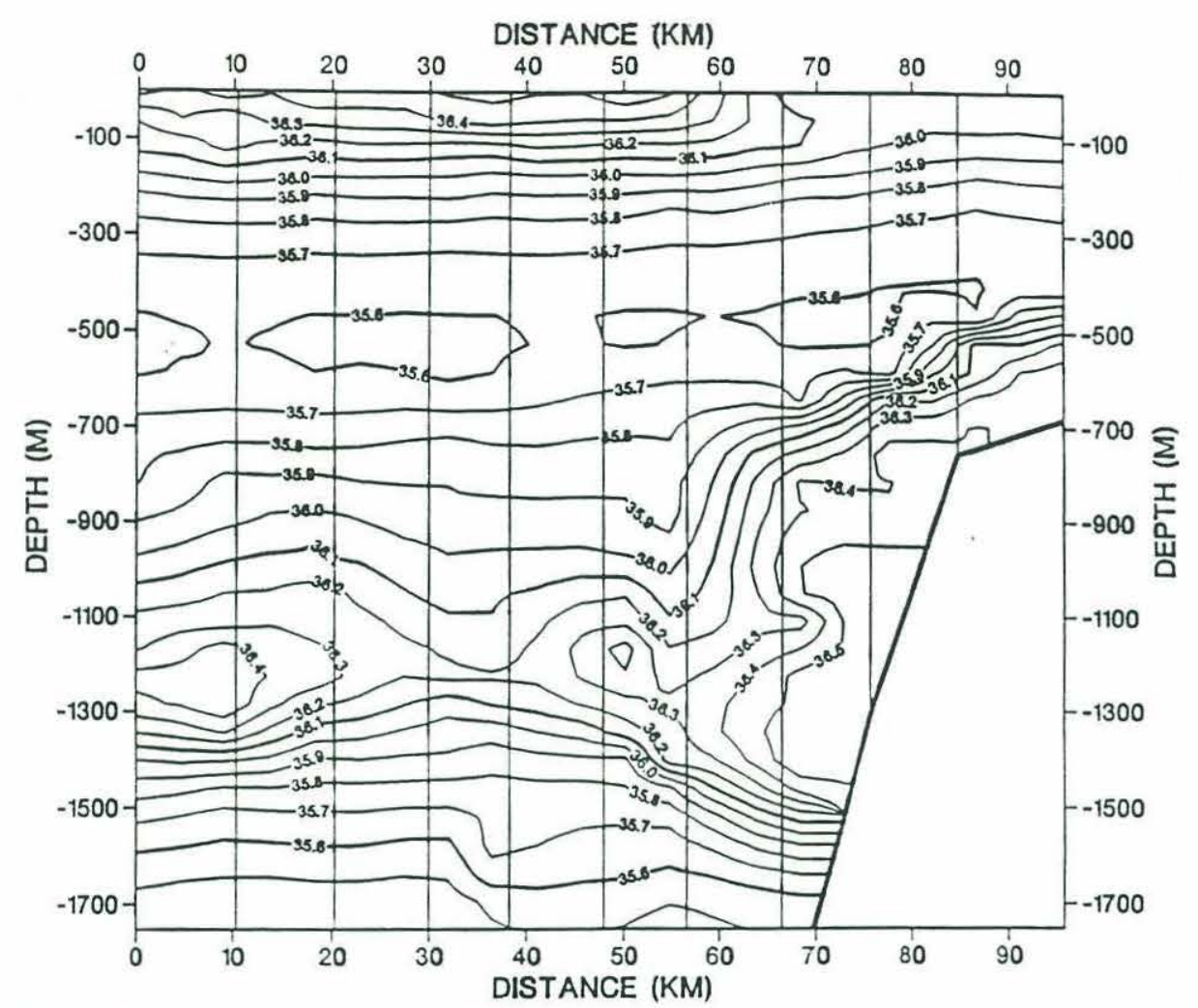

Figure 2.10: Salinity section near $8^{\circ} 30^{\prime} \mathrm{W}$ based on data taken during the 1988 Gulf of Cadiz Expedition (Kennelly et al., 1989b).

\begin{tabular}{|c||c|c|c|c|c|c|c|c|}
\hline & A & B & C & D & E & F & G & H \\
\hline Width & 11.9 & 11.6 & 24.4 & 29.9 & 31.3 & 51.1 & 81.1 & 65.7 \\
\hline
\end{tabular}

Table 2.1: Width of the Mediterranean outflow as defined by the 36.3 isohaline.

of Section G the topographic slope increases and the flow narrows considerably, from $65 \mathrm{~km}$ at section $\mathrm{H}$ to less than $30 \mathrm{~km}$ near Cape St. Vincent (see figure 2.10) although the presence of a Meddy in the southern part of Line 4 makes width estimates ambiguous). 


\section{The spreading of the maximum salinity in plan view}

Figure 2.7(a) gives a plan view of the maximum salinity below the salinity minimum at each station. The 36.5 isohaline and then thr 36.25 isohaline indicate the rapid spreading of the outflow described above. To the north, the 36.25 isohaline, which approximately defines the limits of the Mediterranean outflow plume, follows near the $400 \mathrm{~m}$ isobath, shown in figure 2.7(b, see also figure 2.3). At section $\mathrm{H}$, the northern limit of the outflow descends slightly and follows between the 500 and $700 \mathrm{~m}$ isobath. Low resolution of the station spacing does not allow us to be more precise. In general the northern limit of the outflow decreases from 400 meters at section A to around $600 \mathrm{~m}$ at section $\mathrm{H}$ while the southern edge of the outflow descends from $400 \mathrm{~m}$ to $1350 \mathrm{~m}$.

High salinity values spread along the northern Gulf of Cadiz (figure 2.7(a)). Similar to the composite section along the axis of maximum salinity (figure 2.6), salinity greater than 38.4 is seen in the Strait of Gibraltar. The 37.0 isohaline extends

past section $\mathrm{E}$ and appears to split into two main parts. Part of this high salinity water moves southward across section $\mathrm{FE}$ very near the submarine canyon described by Madelain (1970, figure 1.6). The other part continues past section $\mathrm{F}$ along isobaths, shown in figure 2.7(b). In general the highest salinity water is found on the southern part of each section in the deepest water and is commonly designated the "lower core". The maximum salinity decreases slowly downstream and descends rapidly down the continental slope.

\section{Two cores of salinity?}

Much of the recent work in the Gulf of Cadiz has focused on the development of the double salinity maximum or two cores of Mediterranean outflow. These two cores form two separate subsurface salinity maxima past Cape St. Vincent, tending to 
settle around $750 \mathrm{~m}$ and $1300 \mathrm{~m}$ along the 27.5 and $27.8 \sigma_{\theta}$ surfaces (Zenk $1975 \mathrm{~b}$; Ambar and Howe 1979a). There are two main hypothesis for the subdivision of Mediterranean outflow into two cores. Madelain (1970) concluded that the variable bottom topography and submarine canyons was responsible for subdividing the outflow along different paths (figure 1.6), while Seidler (1968) and later Zenk (1975b) proposed that tidal mixing in the strait produces two distinct peaks in T,S properties that then move out onto the continental slope west of Gibraltar. Howe, Abdullah and Deetea (1974) supported this hypothesis by examining the chemical properties of the two cores and finding correlations between the chemical properties of the upper core and the chemical properties found in the upper Levantine water in the Alboran Sea, while the lower core matched the deeper water found in the Mediterranean.

The GofCExp data suggests that the two distinct cores of outflow evolve through differential mixing within the Gulf of Cadiz. An important distinction between Madelain's steering hypothesis and the results here, is that the horizontal spreading of the outflow plume puts the outflow plume in contact with very different NACW with which it mixes. The cores become increasingly distinct as they separate, spread and mix with different North Atlantic water. We will examine the details of this two core development below.

Although the "lower core" of maximum salinity can clearly be seen, there is less obvious "upper core" present on the maximum salinity map. At section E, for instance, only slight distinctions in outflow properties suggest a minimum salinity of 36.64 in the middle of the section with a slightly larger salinity of 36.77 to the north in shallower water. The minimum salinity in the middle of section $\mathrm{F}$ is even less pronounced with a salinity of 36.75 compared to the salinity of 37.77 of the "upper core". The velocity field shows clearly two distinct "core" of flow at section $\mathrm{E}$ and $\mathrm{F}$ (figure 2.8 and 2.9). At section $\mathrm{E}$ for instance the upper core has maximum $\mathrm{XCP}$ speeds in excess of $40 \mathrm{~cm} / \mathrm{s}$ and stagnant flow separates the lower core which is 
centered at 750 meters depth and has speeds greater than $60 \mathrm{~cm} / \mathrm{s}$. Even though there is no clear separation of the salinity at section $\mathrm{F}$ the velocity field shows a distinct minimum speed of about $10 \mathrm{~cm} / \mathrm{s}$ between the two core velocity signals. These cores are likewise present in the geostrophic velocities at these sections.

By considering the salinity sections shown in figure 2.3 , the two high salinity cores become identifiable at Section E where the Mediterranean outflow separates into two high salinity regions or cores (Figure 2.3(c)). At this longitude, the lower core is following the $760 \mathrm{db}$ isobath with salinity up to 37.51 pss $\left(T=12.94^{\circ} \mathrm{C}\right.$, $\left.\sigma_{\theta}=28.37\right)$. The center of the upper core is much warmer and fresher following the $514 \mathrm{db}$ isobath $\left(S=36.70, T=13.25^{\circ} \mathrm{C}, \sigma_{\theta}=27.67\right)$. Ambar and Howe (1979a) have identified two cores near $7^{\circ} \mathrm{W}$, very close to this section with similar water properties (upper: $T=13.72^{\circ} \mathrm{C}, S=37.07, z=600 \mathrm{~m}$ and lower: $T=13.16^{\circ} \mathrm{C}$, $S=37.42, z=750 \mathrm{~m}$ ). The similarity of these core values and locations is a indication of the general persistence of this outflow.

These two cores can be identified through the other sections as well (see Table 2.2). The initial stages of the water masses separation is barely evident in sections A and B. The saltiest and coldest water in both of these sections is seen on the southern side of the channel. The highest salinity is also found at the deepest section across the Strait. The initial temperatures at the southern and northern stations respectively are only 13.12 versus 13.25 and salinity 38.2 versus 38.0 . By section C there is already a ridge between the two water masses diverting them along separate paths, confirming the importance of topography. The upper core continues along this shallow path while the lower core moves along a deeper isobath. At section $\mathrm{D}$, the maximum salinity appears in the center of the section near the $500 \mathrm{~m}$ isobath and there is no distinct separation of the flow into "cores". It is therefore hard to confirm whether the ridge played a significant role in separating the flow further downstream. When the "cores" finally reach Cape St. Vincent they separate into the North At- 


\begin{tabular}{|c||c|c|c|c||c|c|c|c|}
\hline \multicolumn{1}{l||}{} & \multicolumn{9}{c}{ Lower } \\
Section & $\mathrm{S}$ & $\theta^{\circ} \mathrm{C}$ & $\sigma_{\theta}$ & $\mathrm{P} \mathrm{db}$ & $\mathrm{S}$ & $\theta^{\circ} \mathrm{C}$ & $\sigma_{\theta}$ & $\mathrm{P} \mathrm{db}$ \\
\hline \hline $\mathrm{C}$ & 37.90 & 13.06 & 28.63 & 486 & 37.28 & 13.39 & 28.08 & 494 \\
$\mathrm{E}$ & 37.51 & 12.83 & 28.37 & 760 & 36.70 & 13.18 & 27.67 & 514 \\
$\mathrm{~F}$ & 37.21 & 12.68 & 28.18 & 744 & 36.77 & 12.79 & 27.81 & 672 \\
$\mathrm{G}$ & 36.78 & 12.33 & 27.91 & 992 & 36.62 & 12.40 & 27.77 & 806 \\
$\mathrm{H}$ & 36.57 & 11.87 & 27.83 & 1204 & 36.47 & 12.85 & 27.56 & 758 \\
$\mathrm{~L} 4$ & 36.65 & 12.17 & 27.84 & 1298 & 36.43 & 12.89 & 27.52 & 744 \\
$\mathrm{~L} 8$ & 36.60 & 11.96 & 27.84 & 1366 & 36.44 & 12.67 & 27.58 & 764 \\
\hline
\end{tabular}

Table 2.2: Upper and lower core temperature and salinity values for each section when they are identifiable.

lantic at 750 and $1350 \mathrm{db}$ with core properties $(\mathrm{T}, \mathrm{S})=(12.67,36.44)$ and $(11.96$, $36.60)$.

\section{Velocity weighted salinity maps show two cores}

The horizontal maps of the maximum salinity or temperature along the maximum salinity surface (figure $2.7 \mathrm{a}$ and $2.7 \mathrm{c}$ ) do not reveal the separation of the outflow into cores. However, the velocity fields do suggest a separation. Therefore, we attempt to combine this information by defining a velocity weighted salinity. Define the velocity weighted salinity, $S_{v w t d}$, as,

$$
S_{v w t d}=\frac{\int_{D)}^{D+h} S \vec{v} \cdot \vec{n} d z}{\int_{D}^{D+h} \vec{v} \cdot \vec{n} d z}
$$

where $\vec{n}$ is normal to each section. Physically, this is the equivalent layered salinity of the outflow which represents the dynamically active portions of the flow (see section 2.5.1). Figure 2.11 shows a map of this velocity weighted salinity. Note that this velocity weighted salinity can only be calculated at CTD stations that had concurrent XCP profiles. Therefore, weighted salinities for stations past section $\mathrm{F}$ were not possible. 


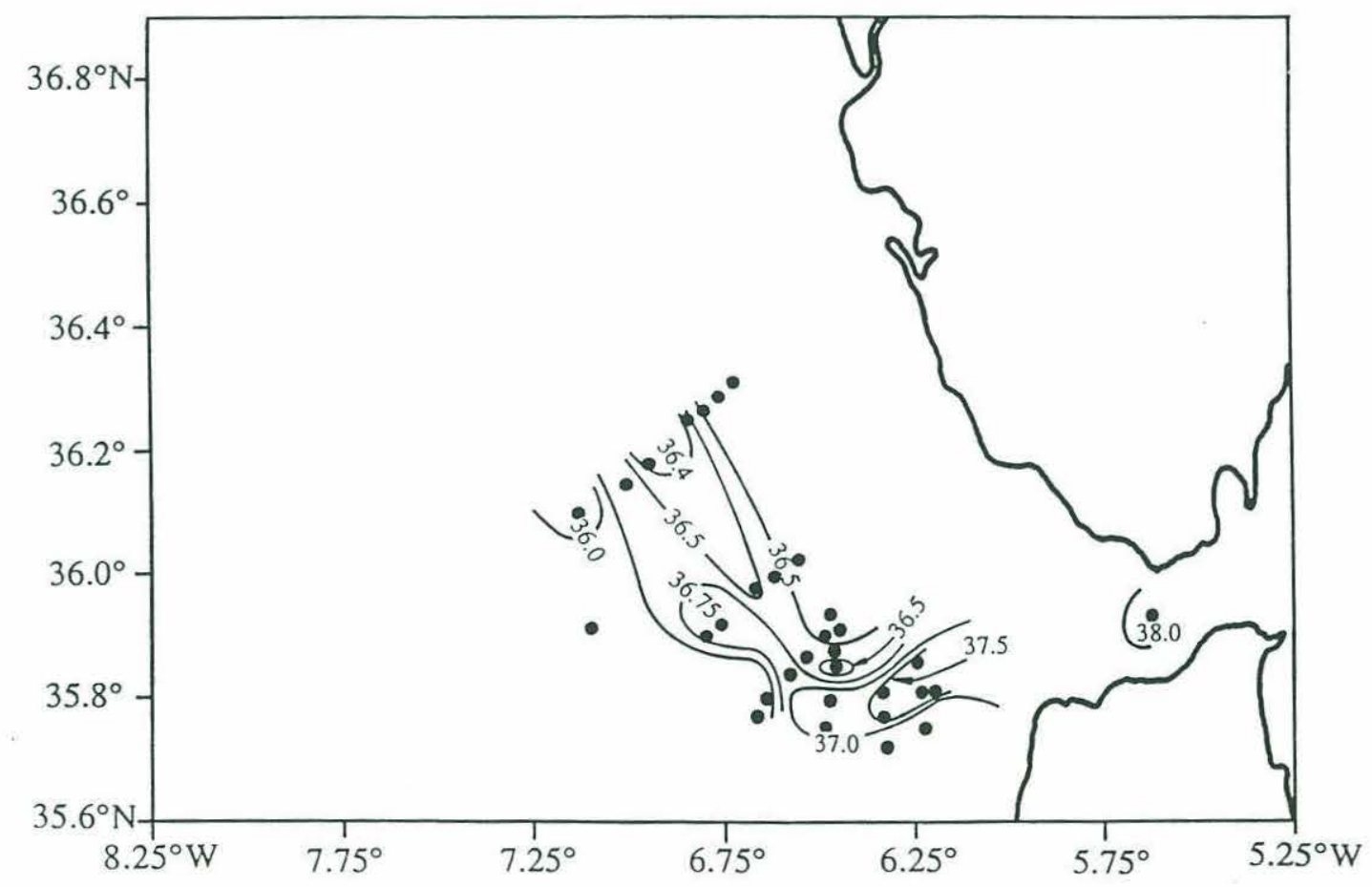

Figure 2.11: Velocity weighted salinity at each station in the Gulf of Cadiz. 
As expected, the two "cores" of high salinity become clearly visible at section $\mathrm{E}$ and section F (figure 2.11). The highest average salinity, $S_{v w t d}$, is again found on the southern, deeper part of each section. The lower core has higher average salinity as well as the highest maximum salinity discussed above. One other striking feature of the velocity weighted salinity map is that the salinity is much lower than the maximum salinity. For instance, the 37.0 isohaline only extends past section $\mathrm{C}$, but the maximum salinity extended past section $\mathrm{F}$ which is $63 \mathrm{~km}$ further downstream. Also, the maximum salinity showed a bifurcation of the lower core past section $\mathrm{E}$, into a component that extended southward to section FE. The $S_{v w t d}$ map, on the other hand, indicates very little salt flux across section FE (as evidenced by the low $S_{v w t d}$ there). We will see later that the total outflow transport at section FE is only $.13 \mathrm{~Sv}$ or less than $10 \%$ of the total outflow transport across section F. This "vein" of flow (as Madelain calls it), is not a very significant portion of the outflow as the $S_{v w t d}$ map suggests. In general, the velocity weighted salinity, $S_{v w t d}$, indicates much more rapid mixing in the outflow plume than the maximum salinity indicates. The implications of the downstream evolution in salinity will be discussed further in the next section.

\section{Water at the salinity minimum}

Strong mixing is occurring along the path of the outflow as it moves through the Gulf of Cadiz. We believe that it is this mixing process that must dominate the final distinction between a lower and upper core because as the flow spreads along the continental slope the outflow is in contact with different NACW. Figure 2.12 shows the potential temperature and salinity of the NACW just above the outflow. The NACW is not uniform above the outflow and horizontal gradients of both temperature and salinity mirror closely the local bottom topography (figure 2.7(b)). Warmer and saltier water is in contact with the upper core while much colder and slightly fresher 
water is available to mix with the lower core. At section F, for instance, the potential temperature of NACW varies from $10.8^{\circ} \mathrm{C}$ to over $13^{\circ} \mathrm{C}$. Although the NACW in contact with the upper core has higher salinity, the increase in temperature makes this water lighter than the NACW that mixes with the lower core. Closer to the strait, the temperature increase and becomes increasingly uniform across each section. Also, the salinity of the NACW increase towards the strait and within the Strait of Gibraltar, salinity values of the NACW exceed 36 .

\section{Temperature and salinity plots}

The temperature versus salinity plots containing stations across each section are most revealing of this separation process. Because the CTD stations and most XCP profiles were concurrent, we can combine these data and present a new form of $\mathrm{T} / \mathrm{S}$ plot. Figure 2.13 illustrates the novel temperature versus salinity diagram for each station with a CTD/XCP drop pair spanning the outflow. The temperature and salinity of every $4 \mathrm{db}$ in a vertical CTD profile is plotted with a symbol whose size is weighted by the horizontal velocity at that depth (therefore, the size of the symbols suggests the speed of the flow, while the number of symbols or density of symbols suggests the amount of water similar to a volumetric T/S plot). For instance in Figure 2.13(a), the largest symbols coincide with the Mediterranean outflow, which is distinguished by its high salinity and rapid westward movement (circular symbols represent westward velocity and triangular symbols for eastward velocity). Notice that the zero velocity for each profile does not coincide with the T,S minimum but is lower in the water column due to the no salt flux requirement imposed.

As you can see from Figure 2.13(a) the T,S properties across the stream diverge further and further downstream. We saw in figure 2.12 that the NACW just above the outflow is always warmer and saltier towards the northern or shallower stations (but lighter). Figure 2.7(c) shows the potential temperature of the outflow at the 

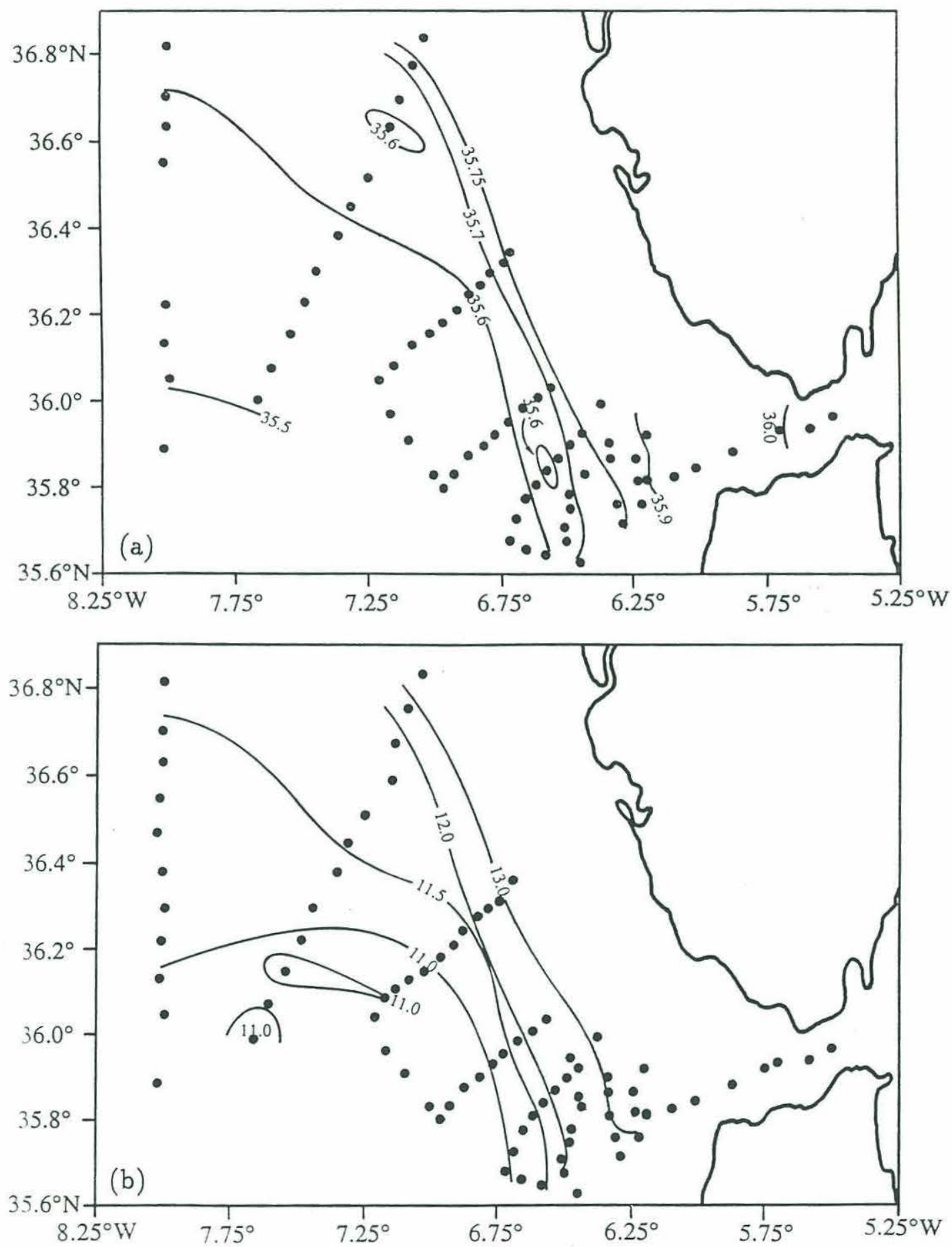

Figure 2.12: Temperature and salinity at each station in the Gulf of Cadiz representing the NACW just above the outflow. (a) NACW salinity. (b) NACW potential temperature. 

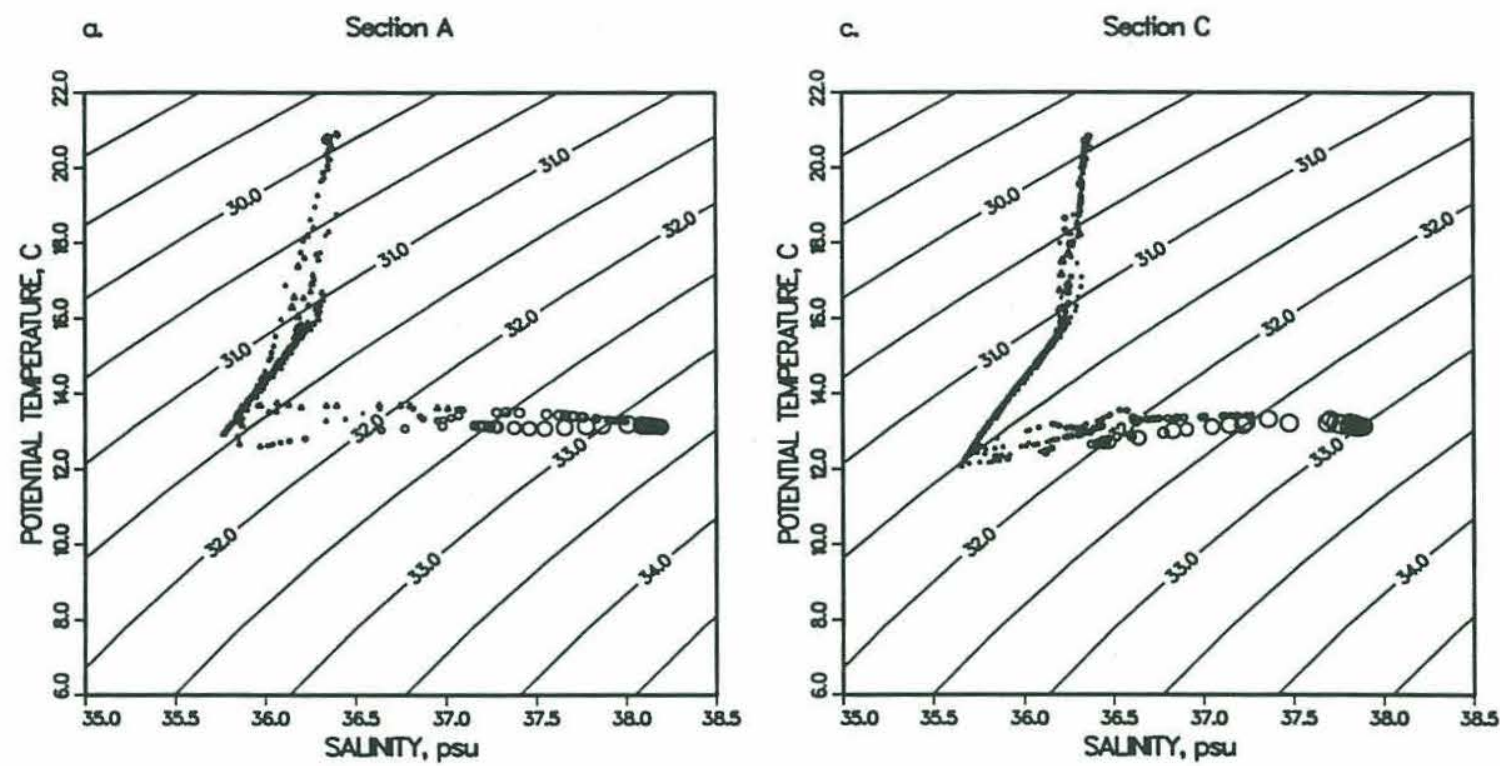

b.

Section B

d

Section D
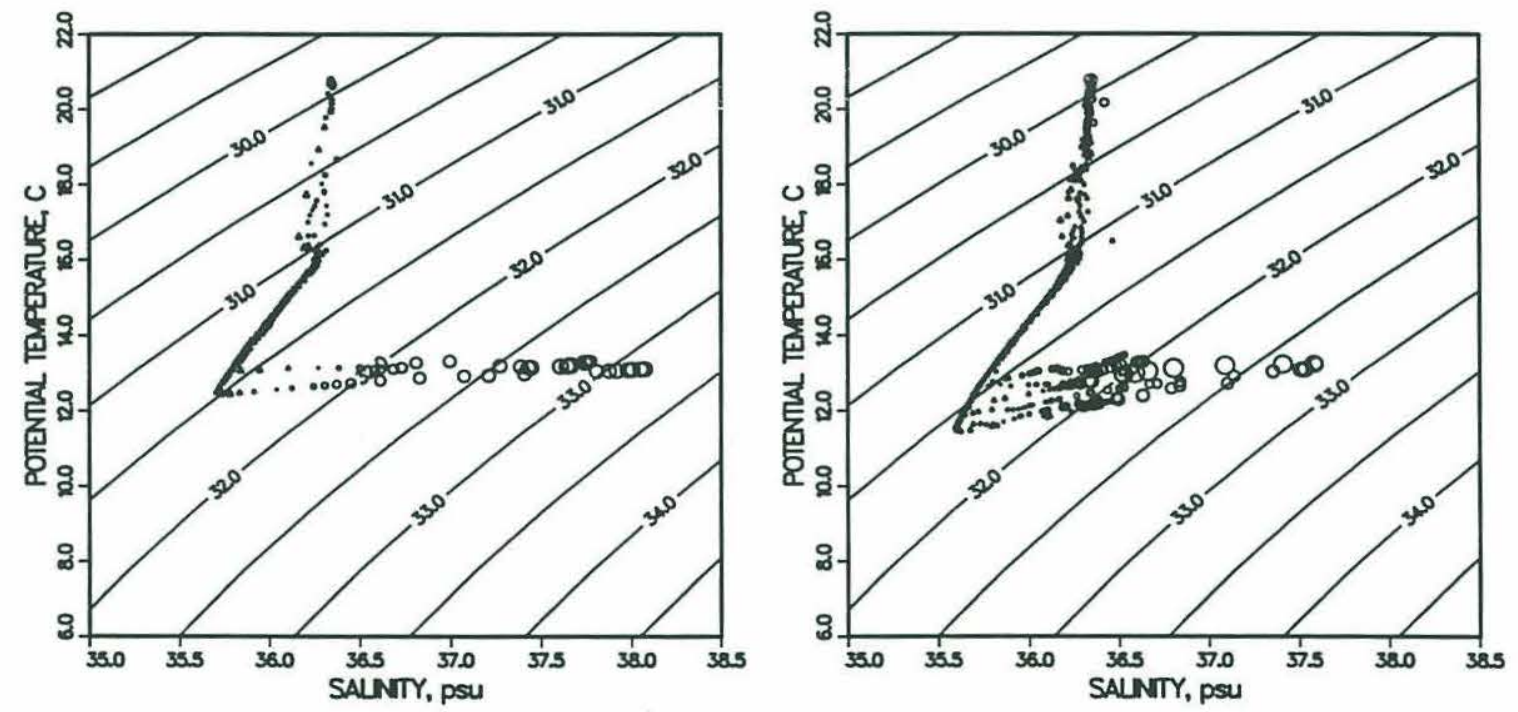

Figure 2.13: Potential Temperature versus Salinity plots. Each station within the outflow with concurrent CTD and XCP profiles is plotted with a symbol representing every $4 \mathrm{db}$ of data. The size of each symbol is scaled by the velocity from the XCP profiler. Circular symbols represent westward moving water and triangular symbols represent eastward moving water. Dashed lines are contours of $\sigma_{\theta}$. (a). Section A, $\mathrm{B}, \mathrm{C}$ and $\mathrm{D}$. 

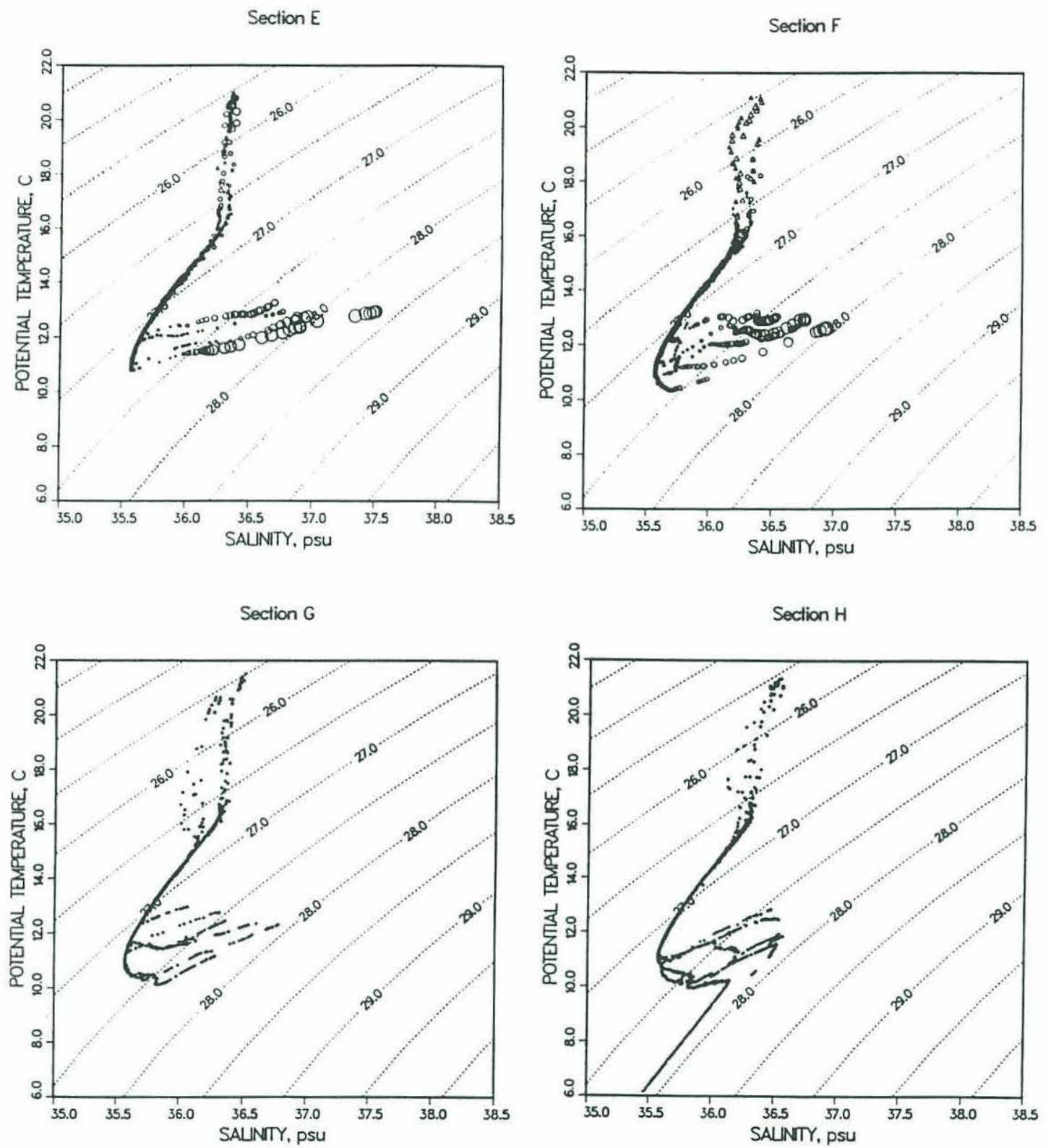

Figure 2.13: (b). Section E, F, G and H. 
depth of the maximum outflow salinity. Like the NACW, the outflow is warmer towards the more northern, shallower stations. The temperature difference across each section is much less than the NACW temperature difference, however. Near the strait the temperature of the outflow at the salinity maximum is fairly uniform, but ranges from $13.1-13.3^{\circ}$ C. Further downstream, this temperature difference increases with, for instance, a range across section $\mathrm{F}$ of $12.5-13.0^{\circ} \mathrm{C}$ (note the even larger temperature differences even further west). Without labeling each station in the temperature salinity plot, we can determine that the coldest saltiest stations are the southern, deeper stations and the stations become progressively warmer and the outflow becomes fresher towards the north.

At section $\mathrm{A}$ and $\mathrm{B}$, it is very difficult to assert that there are two "modes" of water in $\mathrm{T} / \mathrm{S}$ space that are generated in the strait as Seidler suggests. Instead, very fast Mediterranean outflow of very similar water properties seem to emerge from the strait. Further downstream as the flow spreads and mixes with NACW, the Mediterranean water mixes with NACW having different temperatures (notice the different slopes of the $\mathrm{T} / \mathrm{S}$ curves). Assuming that an idealized outflow is uniform in properties as it exists the strait and that the height of the outflow is constant, as the flow spreads along the continental slope, the upper edge of the flow will be higher in the water column than the offshore, deeper edge. If the "background" ocean is stratified and the outflow has a uniform entrainment rate across the flow, then the upper, northern edge of the flow will entrain lighter (i.e. warmer) flow than the lower, southern edge of the flow. The result will be a greater modification of the shallower flow than the deeper flow even though the "entrainment" is uniform. Therefore, even without variations in the outflow properties as Seidler indicates, a uniform outflow would become increasingly nonuniform in cross section as the flow spread and thereby mixed with different NACW. Even without ridges in the topography to steer the flow, this differentiation would continue but perhaps the ridges themselves separate the 
flow sufficiently to form two somewhat distinct relative salinity maxima along the two preferred isopycnals: 27.5 and 27.8 (section $\mathrm{H}$ in figure 2.13(b), Zenk 1975).

\section{$\mathrm{XCP}$ velocity measurements and spreading angles}

The spreading of the outflow has significant implications for the downstream evolution of properties. So how does the flow spread? The outflow appears to have a barotropic and a baroclinic component to this spreading. Figure 2.4(a) shows the maximum velocity at each station containing outflow and figure $2.4(\mathrm{~b})$ shows the velocity of the water 30 dbar above the temperature minimum (which the water closest to the outflow and therefore available for entrainment into the outflow). The maximum velocity shows very strong flow exiting the strait, accelerating to $1.4 \mathrm{~m} / \mathrm{s}$ at section $\mathrm{C}$ where it makes a right hand turn. Downstream of section $\mathrm{C}$, the outflow slows to maximum speeds less than $0.6 \mathrm{~m} / \mathrm{s}$ and aligns itself nearly parallel to the bottom topography. The velocity vectors along any section are not parallel to each other, however. The average outflow velocities at each station indicate a very similar pattern (therefore, not repeated here). The average outflow velocity can be written in polar coordinates and the direction of the velocity at the northern most station can be can be compared to the direction of the velocity of the southern most station containing outflow. For example, at section $\mathrm{D}$ the difference between the northern station and the southern station is 50 degrees. If the outflow were not spreading, this angle would be zero. This angle then, gives a measure of the rate of increase of width downstream. An angle of only 25 degrees would indicate a downstream growth rate of about $\frac{1}{2}$; i.e. $100 \mathrm{~km}$ downstream, the width would increase by $50 \mathrm{~km}$. Note that the actual increase in width from section $\mathrm{C}$ to section $\mathrm{F}$ (or section $\mathrm{G}$ ) occurs at a rate of about $\frac{1}{2}$. An angle as large as 50 degrees would indicate greater than a one to one growth of the width: i.e. $100 \mathrm{~km}$ downstream the width would increase $120 \mathrm{~km}$ ! Section D is an extreme example, however. At section $\mathrm{E}$ the average angle is only 34 degrees and 
at section F, even less. Very small horizontal differences in the direction of the flow could easily account for the observed spreading of the outflow.

The velocity field also suggests considerable baroclinic structure. Each of the velocity profiles shown in figure 2.15 contains a significant veering of the velocity profile through the transition layer between the salty, fast, westward flowing Mediterranean water and the NACW above it. The veering in the outflow layer can be visualized better by looking at a horizontal map of velocity at two different depths. Figure 2.16 shows the velocity 10 dbar above the bottom (solid arrow heads) and the velocity 40 dbar above the bottom (open arrow heads). Very few of these vector pairs are parallel. Most stations containing westward moving Mediterranean outflow have the deepest vector directed downslope relative to velocity vector $40 \mathrm{dbar}$ above the bottom. This is what you would expect in a bottom Ekman layer. Only three exceptions are noted, all located at the southern stations along section $\mathrm{B}, \mathrm{C}$ and $\mathrm{E}$. In all three of these cases the flow have complicated bathymetry features. At section B and $\mathrm{C}$ the flow is in the channel and at section $\mathrm{E}$ this station is in a slight depression in the topography (i.e. the station just to the south is shallower).

The downslope component of the deepest velocity in figure 2.16 is suggestive of a frictional bottom boundary layer. A classical Ekman layer would have a downslope or southward velocity in the bottom boundary layer. On the other hand, for a frictional interfacial layer, there would be a northward deflection of the velocity in this layer above the 'free stream' geostrophic velocity in the lowest layer. The full $\mathrm{XCP}$ profiles, as shown in figure 2.15 , suggest that the veering in the interfacial or internal "boundary layer" dominates. One way to examine the vertical structure of the outflow is to assume that the outflow can be divided into two layers: below the velocity maximum and above the velocity maximum. The average velocity above and below the velocity maximum can then be calculated for each station. An ensemble average of the the angle between the average velocity above the velocity maximum 

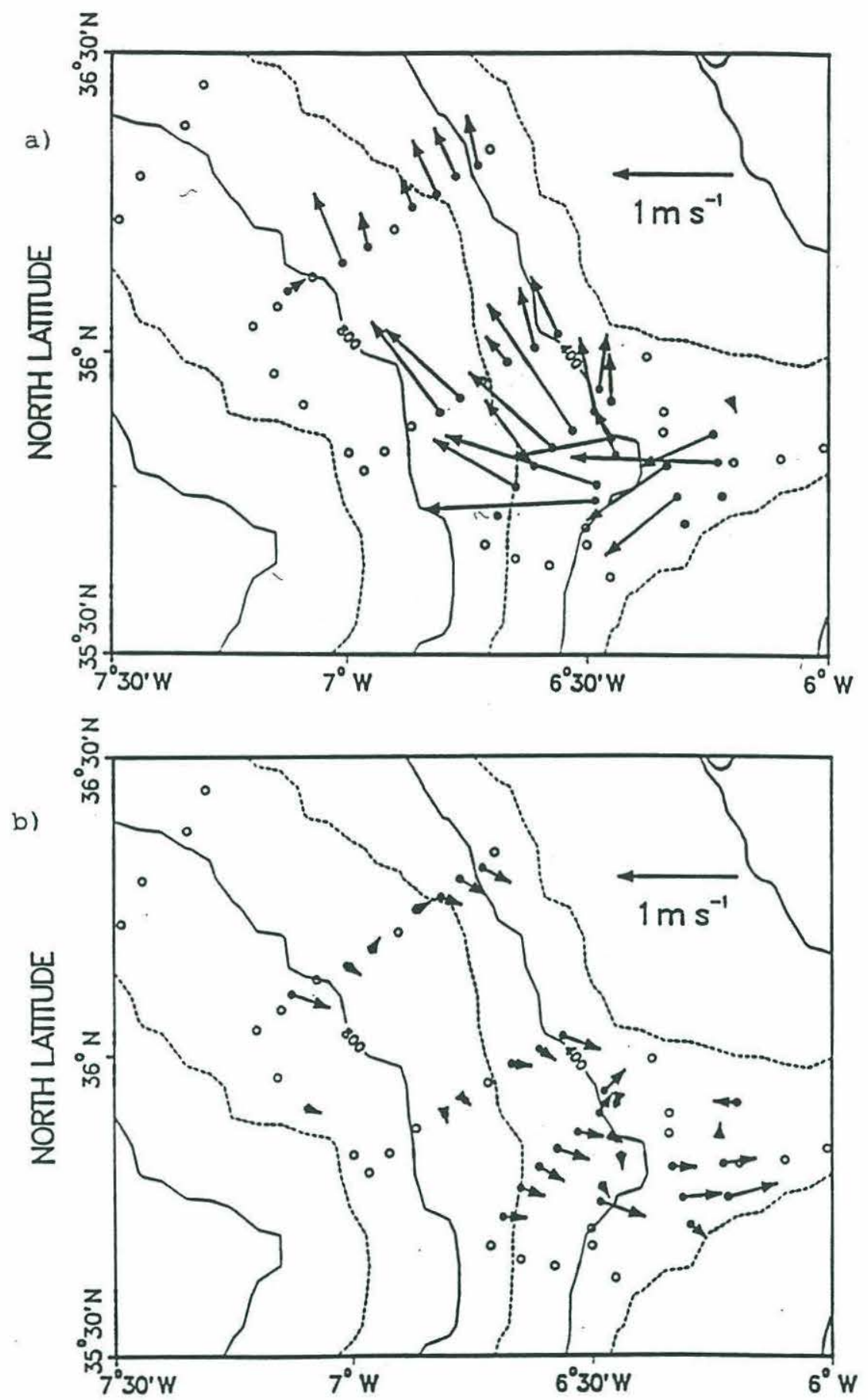

Figure 2.14: Maximum outflow XCP velocity and the XCP velocity at the depth of the salinity minimum. Topographic maps of the Gulf of Cadiz with contours of the 400 and $800 \mathrm{~m}$ isobaths with solid dots for stations with XCPs and open dots for stations where no XCP was present. (a) the maximum outflow velocity for each XCP. (b) the water $30 \mathrm{db}$ above the T,S minimum at each station. This water is assumed to be entrained into the outflow. 


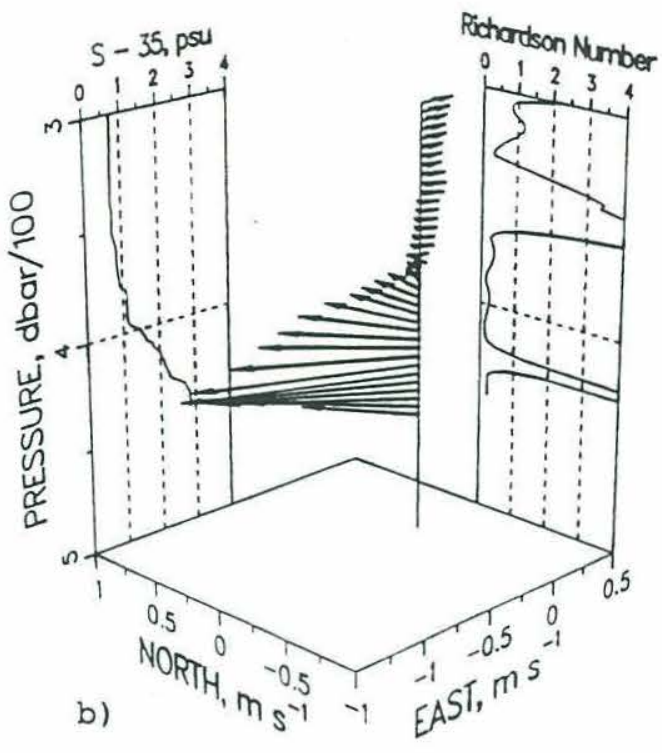

CTD86, XCP54, SECTION D

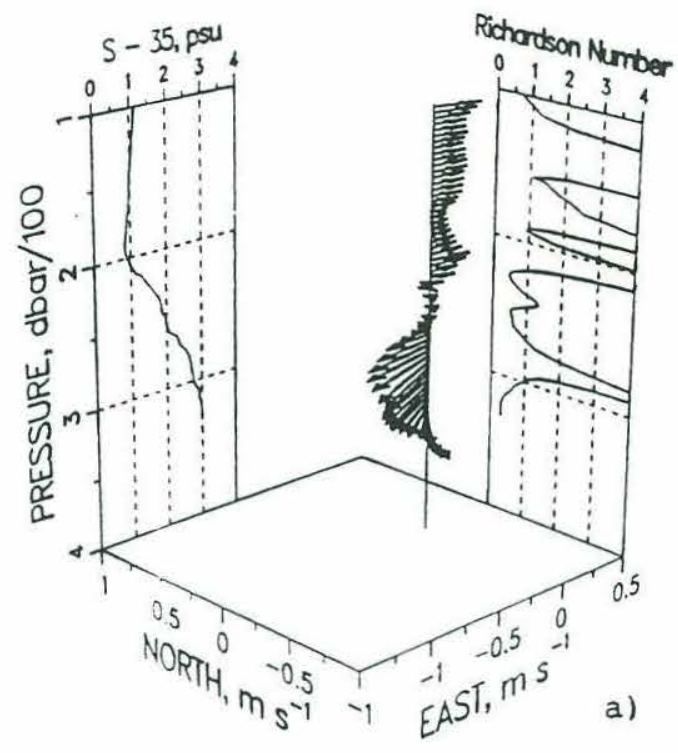

CTD83, XCP51, SECTION D
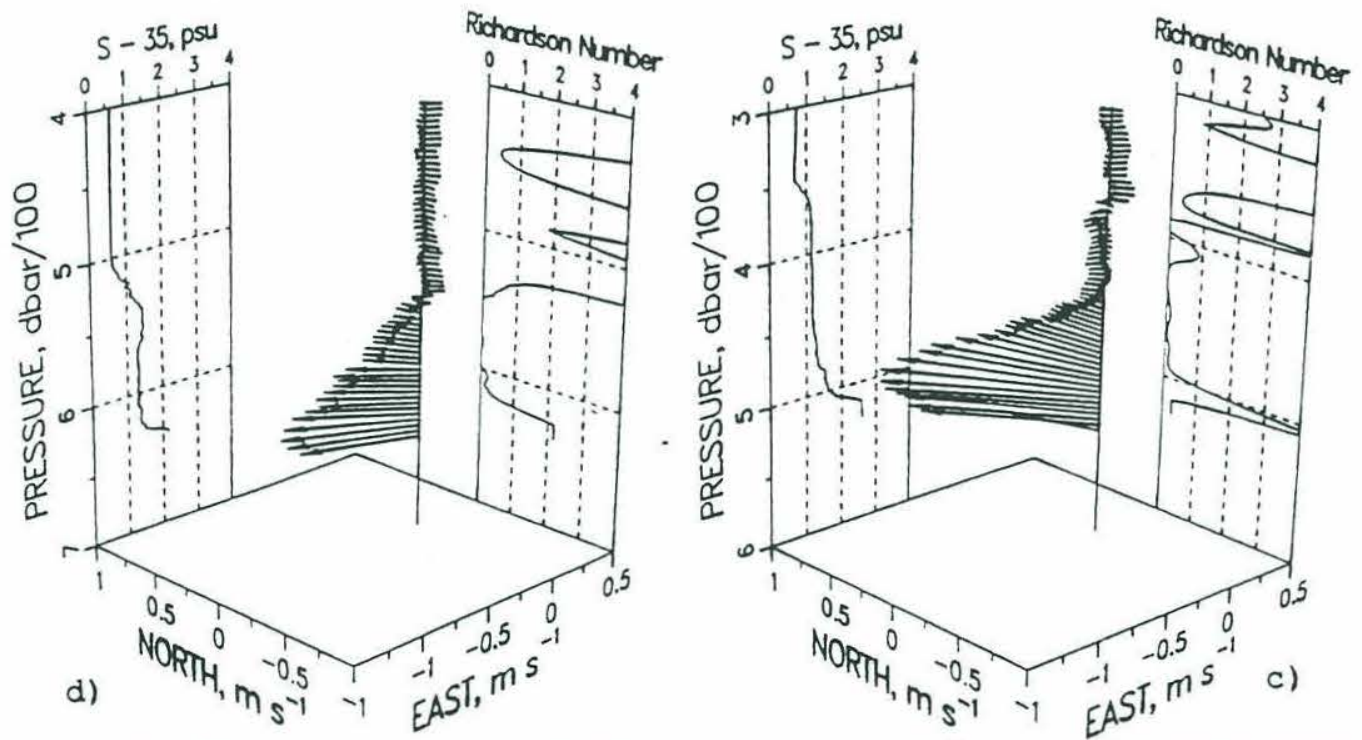

Figure 2.15: Three dimensional XCP velocity vector diagram for CTD/XCP drop pairs. The XCP velocity versus depth is plotted for every 4 meters of data. The corresponding salinity anomaly and gradient Richardson number versus depth are also plotted. a). CTD 65 contains salty Mediterranean water below $200 \mathrm{db}$ moving towards the SW. Low gradient Richardson numbers are found in the interface and the well mixed bottom layer. b). CTD 76 contains salty Mediterranean water moving towards the NW with critical gradient Richardson Numbers. c). CTD 83 is an example of the layered structure of the outflow. d). CTD 86 has an unstable density profile suggesting mixing. 


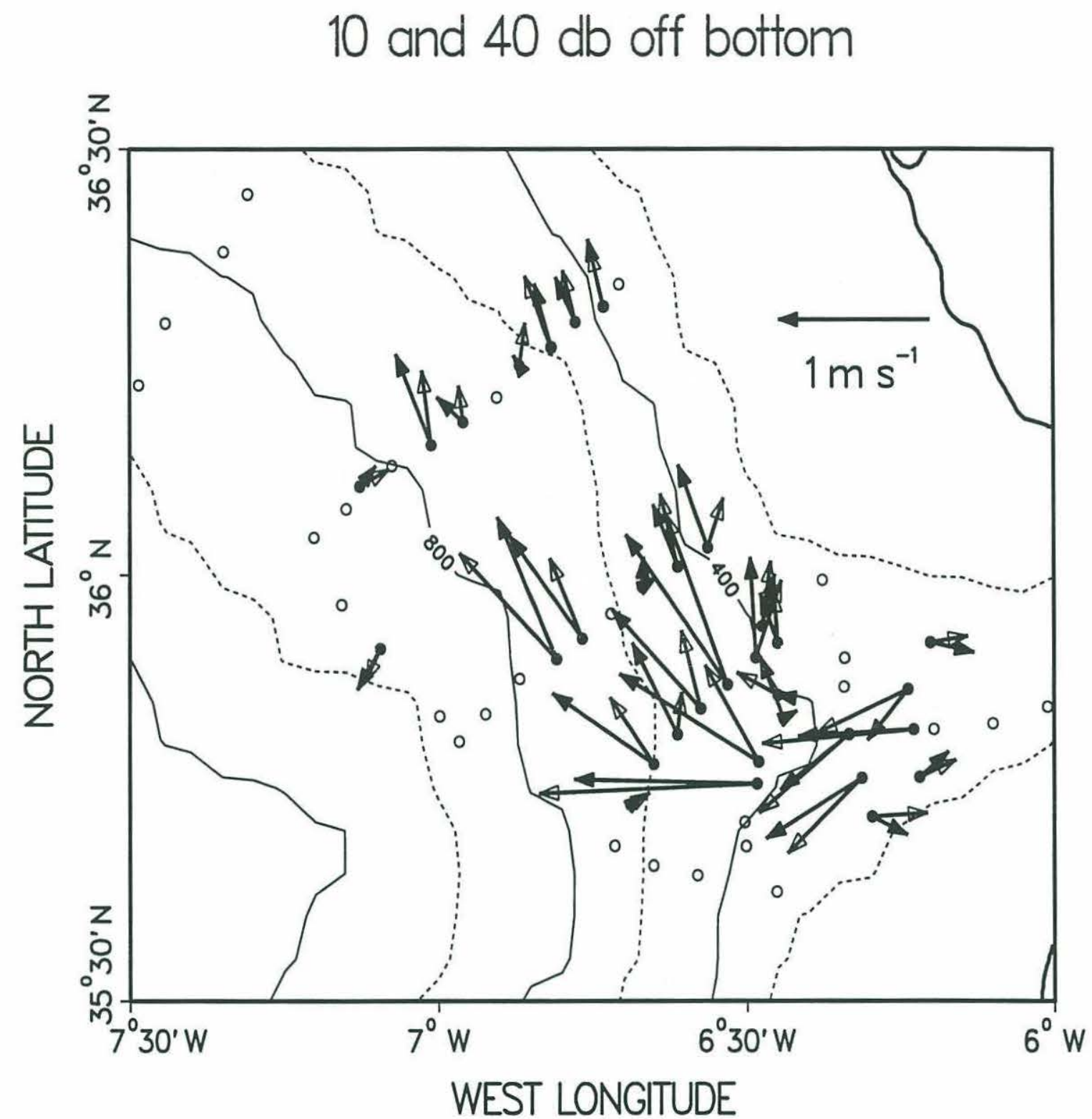

Figure 2.16: Velocity map 10 and $40 \mathrm{db}$ above the bottom. Topographic maps of the Gulf of Cadiz with contours of the 400 and $800 \mathrm{~m}$ isobaths, solid dots for stations with XCPs and open dots for stations where no XCP is present. The velocity $10 \mathrm{db}$ above the bottom is represented by solid arrow heads. The velocity $40 \mathrm{db}$ above the bottom is represented by open arrow heads. 
and the velocity maximum itself gives an angle of $5.3 \pm 0.4$ degrees ( \pm the standard error, positive means to the north relative to the velocity maximum). The average angle below the velocity maximum is $-2.8 \pm 0.3$ (negative means to the south or counterclockwise with depth). Neither of these mean values is significantly different from zero, but both do support our feeling for the observed veering seen in the XCP profiles and figure 2.16. One reason they are so low is that the velocities are averages: the angle of any two velocity vectors (i.e. specific depths as shown in figure 2.16), would be much larger. The average velocities do suggest an average angle between the 'upper' portion of the outflow and the 'lower' portion of the outflow of about 8 degrees. This angle suggests a spreading rate of only 0.14: i.e. in $100 \mathrm{~km}$ the width would increase only $14 \mathrm{~km}$. Although this is a relatively small spreading rate, the influence of the baroclinic structure on the spreading cannot be ignored, especially when instantaneous velocities are considered. 


\subsection{Entrainment}

The Mediterranean outflow plume undergoes considerable change as it descends along the continental slope of the Gulf of Cadiz and transitions to the Mediterranean outflow tongue in the North Atlantic. In section 2.4, we saw how the Mediterranean outflow salinity decreases from 38.4 pss in the Strait of Gibraltar to 36.5 pss in the Gulf of Cadiz as it descends the continental slope (Figure 2.7(a)). To understand the change in the salinity of the outflow we need to first quantify the entrainment into the outflow. In this section, we will first examine the changes in the bulk properties of the flow and the entrainment, then in section 2.5.2 we will ask the question: is there a Froude number or Richardson number dependence to the entrainment?

\subsubsection{Observations}

Mixing between the Mediterranean outflow and North Atlantic Central Water (NACW) will be examined by considering the evolution of the average $(\mathrm{T}, \mathrm{S})$ properties. Entrainment will be calculated by the downstream divergence or convergence of the outflow transport. These methods are different because one deals with the property field which is established over a long time scale and the other method deals with the velocity field more directly and is therefore more sensitive to tidal and sub-tidal fluctuations. Note that if the Mediterranean outflow were truly steady, the absolute velocity field could be chosen so that the transport and salt flux gives the same entrainment estimates (see section 2.5.1). These two different estimates of entrainment will be reconciled. The terms entrainment and mixing will often be used interchangeably because they are closely related. Generally, entrainment refers to the outflow increasing its volume transport and mixing refers to the accompanying change in properties. 


\section{Downstream evolution of core properties}

The downstream evolution of properties is a good indicator of mixing. The maximum salinity, $S_{\max }$ a, in the outflow layer for each station is shown in figure 2.6. As with the composite section along the axis of the flow (figure 2.6(c)), this map illustrates the rapid decrease in $S_{\max }$ as the outflow descends the continental slope. Salinity as large as 38.2 pss is found in section A while the salinity has decreased to 37.5 pss at section $\mathrm{E}$ ( $65 \mathrm{~km}$ away). The 37.0 isohaline extends to section $\mathrm{F}$ and a small portion of this salty water seems to move further downslope flowing southwest at section FE. The saltiest water is clearly downslope or further offshore and is associated with what has historically been called the lower core (see section 2.4).

\section{Downstream evolution of salt flux}

In section 2.4 , we defined the velocity weighted salinity, $S_{v w t d}$, and found that this representation of salinity revealed the presence of two distinct cores (see figure 2.11), while the $S_{\max }$ figure 2.7 shows only broad spreading. Similarly, the $S_{v w t d}$ map shows a much more rapid decrease in the salinity of the outflow as it moves into the Gulf of Cadiz. The $S_{\text {vwtd }} 37.0$ isohaline only extends to section C, $60 \mathrm{~km}$ closer to the strait than the $S_{\max } 37.0$ isohaline. Thus, $S_{v w t d}$ indicates much more vigorous, localized

mixing than $S_{\max }$. Historically, people have used the 'core' properties to estimate entrainment into the outflow which has lead to gradual mixing along the path. Here, we find the salt flux (equivalently $\bar{S}$ and $S_{v w t d}$ ) indicates that the mixing is very intermittent or localized along the path.

To consider the outflow as whole, we will use the transport weighted salinity across each section. We define the transport weighted salinity, $\bar{S}$ as,

$$
\bar{S}=\frac{\int_{0}^{W} \int_{D)}^{D+h} S \vec{v} \cdot \vec{n} d z d y}{\int_{0}^{W} \int_{D}^{D+h} \vec{v} \cdot \vec{n} d z d y}
$$


where the bottom topography is $-D(x, y), \mathrm{h}(\mathrm{x}, \mathrm{y})$ is the top of the outflow, and $W(x)$ is the width of the outflow. This form of average salinity has the advantage of representing that part of the flow which is dynamically active. It represents what the average salinity would be if the outflow consisted of a single constant velocity layer with the same salt flux. $\bar{S}$ is the appropriate measure of the salinity to compare with a simple two layer models. We could discuss salinity fluxes directly, but we choose to use $\bar{S}$ because 1 ) we can compare this salinity with the maximum values and the overlying NACW and 2) historically the maximum salinity has been used as an indicator of mixing (Zenk, 1975, Ambar and Howe 1979). Note that when we actually calculate an entrainment rate in section 2.6 .4 we will use the salt flux. We will examine the flux of 'pure' Mediterranean water in section 2.5.1.

Figure 2.17 illustrates $\bar{S}$ at each section as a function of downstream distance. Note that along the strait no section average was possible so $S_{v w t d}$ was plotted. $\bar{S}$ decreases downstream from 37.8 pss to $36.7 \mathrm{pss}$ in the $40 \mathrm{~km}$ from section $\mathrm{A}$ to $\mathrm{D}$. The greatest change occurs near the Strait before section D, suggesting large entrainment between these sections. This figure also indicates the maximum salinity found at each section (i.e. the maximum $S_{\max }$ for each section). As with the maximum salinity map (figure 2.7), the core values suggest gradual mixing over many kilometers while $\bar{S}$ shows localized, intense mixing before section D. Clearly, these two measurements indicate very different entrainment rates.

\section{Downstream evolution of transport}

Entrainment into the overflow can also be diagnosed by examining the downstream change in the outflow transport. Transport for each section is calculated using both the XCP velocities and geostrophic velocities using the referencing scheme described in Section 2.3. The total outflow transport is calculated by assuming that the outflow lies entirely on the bottom and is bounded by the zero velocity surface. Where the 


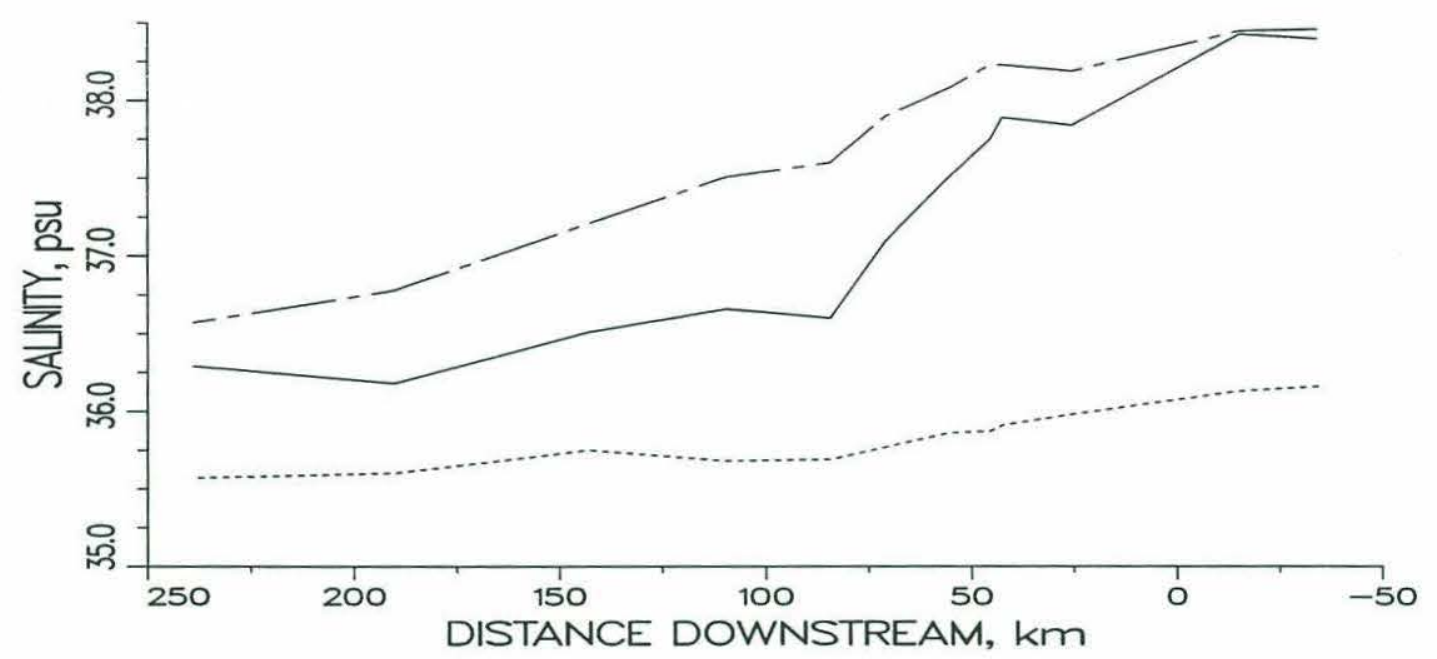

Figure 2.17: Salinity versus downstream distance. The maximum salinity across each section (the dotted line), gradually decays downstream while the velocity weighted salinity (the solid line), decays rapidly within the first 40 kilometers from section A. The dashed line represents the salinity of the overlying North Atlantic water.

outflow is separated from the bottom at sections $\mathrm{G}$ and $\mathrm{H}$, the transport estimate includes everything moving westward below the reference level.

Figure 2.18 shows the resulting total outflow transport for each section. In general the XCP transports match fairly well the geostrophic velocity transport estimates. This is rather surprising given the expected ageostrophic nature of the current, particularly near the Strait of Gibraltar. For instance, section $\mathrm{C}$ has a very low geostrophic transport of only $0.2 \mathrm{~Sv}$ which doesn't match the XCP transport of $1.0 \mathrm{~Sv}$. A break down in geostrophy and sampling errors due to the poor spatial coverage are responsible for the poor correspondence there (see section 1.6.3). Both geostrophic and XCP transport estimates indicate an increase from $0.8 \mathrm{~Sv}$ to $1.2 \mathrm{~Sv}$ in $40 \mathrm{~km}$ with very little increase beyond. This increase in outflow transport suggests an entrainment of $0.4 \mathrm{~Sv}$ between sections B and D and little entrainment thereafter. Note that the change in transport downstream is similar for other reference 


\section{VOLUME TRANSPORT}

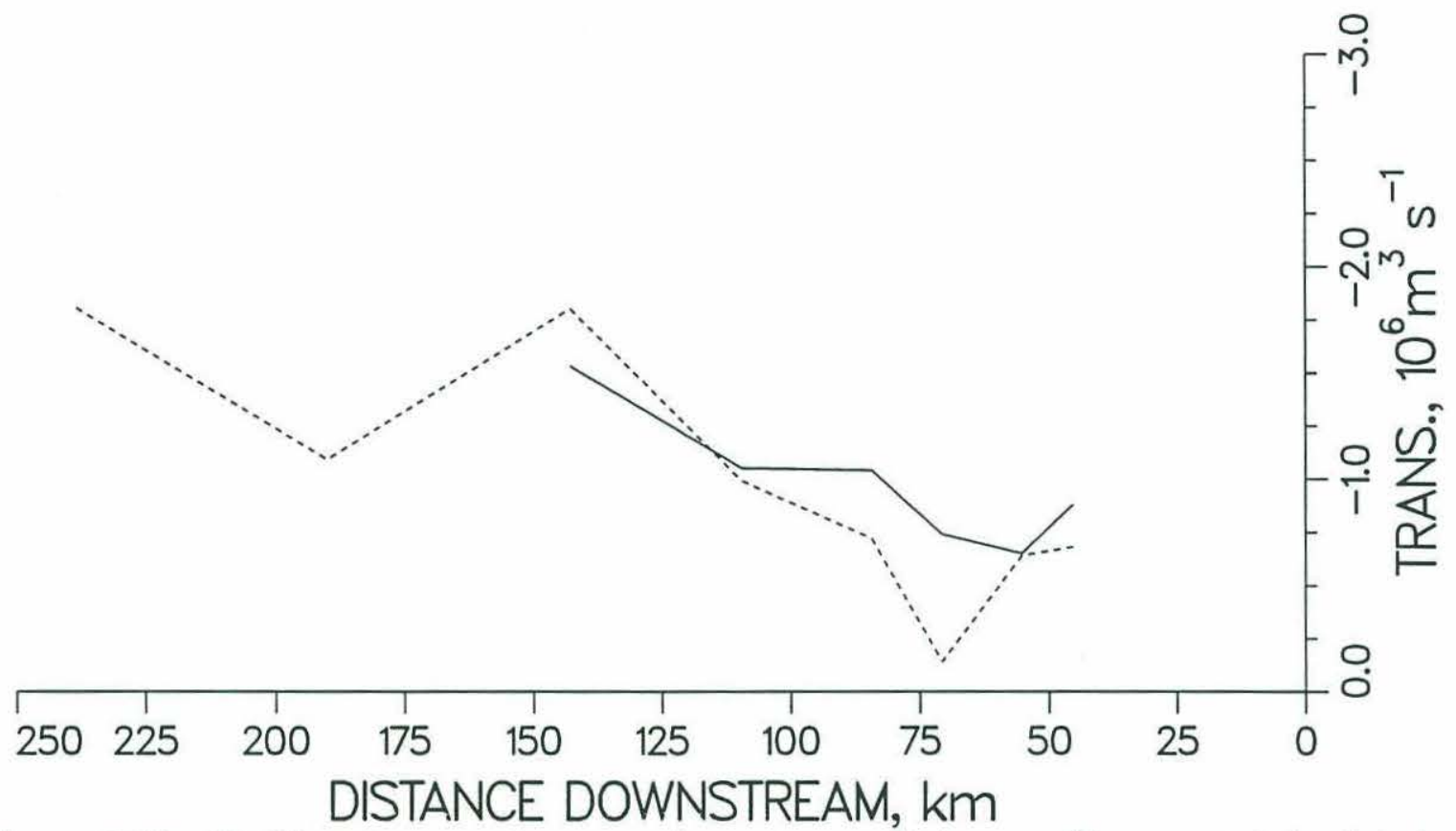

Figure 2.18: Outflow transport versus downstream distance. Transport calculated from XCPs (solid line) and transports calculated from geostrophic velocities (dashed line) both have similar magnitudes with a rapid increase in outflow transport after section $\mathrm{C}$.

level choices (not shown) indicating that the implied entrainment is insensitive to the reference level chosen.

\section{Transport of pure Mediterranean water}

The transport weighted salinity, $\bar{S}$, suggests entrainment between section A and section $\mathrm{D}$ while the transport estimates indicate entrainment between section $\mathrm{C}$ and $\mathrm{F}$. At first glance the fluxes of mass and salt seem to be telling us contradictory stories. We know that some volume of pure Mediterranean water, $Q_{m e d}$, exits the Strait of Gibraltar with a salinity of 'pure' Mediterranean water, $S_{m e d}=38.4$ pss. Ultimately 
this 'pure' Mediterranean water must cross each of our hydrographic sections along with the water of NACW that the outflow has entrained from above.

We can reconcile this apparent contradiction by considering the simplest possible mixing argument. Assume that the outflow water is entraining water of salinity 35.6 pss (i.e. the approximate salinity at the base of the Atlantic water, Zenk 1975a) and the outflow water at the Camarinal sill has an average salinity of 38.4 pss (Figure 2.6). Requiring conservation of salt means

$$
\bar{S} Q_{\text {out }}=S_{\text {med }} Q_{\text {med }}+S_{N A} Q_{\text {ent }},
$$

where the total plume transport $Q_{\text {out }}$ is the sum of the outflow transport, $Q_{\text {med }}$, and the entrained North Atlantic water, $Q_{\text {ent }}$. Then assume conservation of mass, so that this equation can be rewritten as,

$$
Q_{\text {med }}=\left[\frac{\bar{S}-S_{N A}}{S_{m e d}-S_{N A}}\right] Q_{\text {out }} .
$$

This equation is overdetermined. $Q_{\text {ent }}$ and $Q_{\text {med }}$ can be estimated provided we specify $S_{m e d}$ and $S_{N A}$, and calculate $Q_{o u t}$ and $\bar{S}$ from the observations.

Table 2.3 lists the estimate of $Q_{m e d}$ and the total entrainment from the strait estimated from equation 2.4. If the Mediterranean outflow transport is steady, ${ }^{6}$ a fixed flux of Mediterranean water exits the Strait. Therefore, the flux of 'pure' Mediterranean water should be conserved across each section as the flow moves downstream. Sections B, C, D and E all have similar inferred fluxes of Mediterranean water of $0.4 \mathrm{~Sv}$. For these sections it appears that the flux of Mediterranean water is conserved and that the transport estimates are consistent with the average salinity. Table 2.3 also says that the entrainment occurs primarily between sections $\mathrm{C}$ and $\mathrm{D}$ and sections $\mathrm{E}$ and $\mathrm{F}$. We will see in section 2.6.4 that these entrainment estimates allow us to infer the entrainment rate and the entrainment stress. Section A suggests

\footnotetext{
${ }^{6}$ Recall that the velocity at Spartel West fluctuated by $15 \%$ (Armi and Farmer, 1988), so this steady assumption is only accurate to this extent.
} 


\begin{tabular}{|c|c|c|c|c||c|c||c|c|}
\hline & & & \multicolumn{1}{|c||}{} & \multicolumn{4}{c|}{$Q_{\text {med }}=.4 S v$} \\
Section & $Q_{\text {obs }}$ & $Q_{\text {med }}$ & $Q_{\text {ent }}$ & $\bar{S}$ & $S_{\text {ent }}$ & $Q_{\text {ent }}$ & $Q_{\text {mod }}$ & $Q_{\text {ent }}$ \\
\hline \hline A & -0.88 & -0.68 & 0.20 & 37.75 & 37.2 & 0.48 & 0.52 & 0.12 \\
B & -0.65 & -0.44 & 0.21 & 37.51 & 36.1 & 0.25 & 0.61 & 0.21 \\
C & -0.74 & -0.40 & 0.34 & 37.10 & 35.6 & 0.34 & 0.74 & 0.34 \\
D & -1.04 & -0.37 & 0.67 & 36.60 & 35.5 & 0.64 & 1.07 & 0.67 \\
E & -1.07 & -0.40 & 0.67 & 36.65 & 35.6 & 0.67 & 1.05 & 0.65 \\
F & -1.40 & -0.47 & 0.93 & 36.54 & 35.8 & 1.00 & 1.19 & 0.79 \\
F + FE & -1.53 & -0.50 & 1.03 & 36.51 & 35.8 & 1.13 & 1.23 & 0.83 \\
\hline
\end{tabular}

Table 2.3: Flux of Mediterranean water, $Q_{\text {med }}$, for each section determined through a simple mixing argument. $Q_{o b s}$ is the total outflow transport, $Q_{\text {ent }}$ is the estimate of entrained NACW (that mixes with 'pure' Mediterranean water), and $\bar{S}$ is the transport weighted salinity defined in equation 2.2 . Note section ' $F+F E$ ' is a combination of transport from section $\mathrm{F}$ and section $\mathrm{FE}$ and is done because whatever outflow passes section $\mathrm{E}$ must flow past sections $\mathrm{F}$ and $\mathrm{FE}$ : section $\mathrm{F}$ does not capture all of the outflow.

a high $Q_{\text {med }}$ of $0.68 \mathrm{~Sv}$. This means that a large flux of pure Mediterranean water is required to create the observed high flux of salinity at section A. Section F and section FE combined suggest a high flux of Mediterranean water of $0.51 \mathrm{~Sv}$.

This calculation illustrates that for sections $\mathrm{B}$ through $\mathrm{E}$ the temperature minimum NSF reference level flow conserves Mediterranean salt flux, as expected if the outflow is steady. Section A and section F both suggest the flux of more Mediterranean salt. Rewriting equation 2.4 slightly, the total outflow transport that is implied by a given $S_{\text {out }}$ and $Q_{\text {med }}$ can be determined. Assuming a flux of $0.4 \mathrm{~Sv}$ of pure Mediterranean water and the same average salinity, $\bar{S}$, produces outflow transports of 0.52 and $1.23 \mathrm{~Sv}$ for section $\mathrm{A}$ and $\mathrm{F}$ respectively compared to the observed estimate of .88 and $1.4 \mathrm{~Sv}$. The modified transport estimate, $Q_{\text {mod }}$, is listed in the last part of table 2.3. The difference of the transport estimates for section $\mathrm{F}$ is certainly within the range of uncertainty of the original calculation. Section A and B have potentially much larger transport uncertainties for two reasons. Both Section A 
and $B$ have only two stations located within the outflow, therefore sampling problems alone could bias our transport estimates. These sections are also close to the strait and could have true time dependent fluctuations. Armi and Farmer (1991) have shown that time dependent current fluctuations are only $20 \%$ at Spartel West where section B is located. Current variations coupled with interface height undulations should be even larger at section $\mathrm{A}$.

Another possibility is that the salinity of the entrained water is not 35.6. In figure 2.12(b) and figure 2.17 we saw that the minimum salinity just above the outflow layer was higher closer to the Strait of Gibraltar. We can turn the above calculation around again by rewriting equation 2.4 as,

$$
S_{\text {ent }}=\left[\frac{\bar{S} Q_{o u t}-S_{m e d} Q_{m e d}}{Q_{\text {out }}-Q_{m e d}}\right] .
$$

Fixing $Q_{m e d}$ at $0.4 \mathrm{~Sv}$ and $S_{m e d}$ at 38.4 we can then estimate the required salinity of the entrained water, shown in table 2.3. $S_{\text {ent }}$ is 37.2 pss between section A and the Mediterranean. Note that Bryden, Brady and Pillsbury (1989) found that the mean along strait velocity was zero at the 37.0 isohaline at Camarinal Sill. So it is reasonable to assume that the Atlantic water that is being entrained before section $\mathrm{A}$ has a very high salinity. The high $Q_{\text {med }}$ at section A obtained in the first calculation appears to be due to some combination of time dependence and failure to specify $S_{\text {ent }}$ properly.

\section{Vertical structure of the transport}

Figure 2.19(a) illustrates the basic observables of the flow we have examined. Transport at each section is averaged into $20 \mathrm{~m}$ depth intervals. The outflow transport is shaded and is shown descending the continental slope. The inflow transport is unshaded and is largest at mid depth. Near the surface the 'inflow' reverses direction and at some sections is actually moving westward in the same direction as the outflow. 
The flow is conceptually divisible into three parts: a constant depth between sections $\mathrm{A}$ and $\mathrm{B}$, a steep descent between section $\mathrm{B}$ and $\mathrm{E}$, and a gentle descent downstream of section $\mathrm{E}$.

Along the shelf between sections A and B, the flow mixes very little with the overlying NACW but does mix internally. We saw that the maximum salinity and the velocity weighted salinity both decreased in this region. This internal mixing could be driven by bottom generated turbulence that mixes the stratified outflow raising its center of gravity, without driving entrainment at the interface. This is confirmed by the lack of increased entrained water through section C shown in table 2.3.

Along the steep descent, the maximum salinity, $S_{v w t d}$, and $\bar{S}$ of the outflow continue to decay as the flow moves westward. The outflow transport increases between section $\mathrm{C}$ and $\mathrm{D}$, entraining an additional $0.33 \mathrm{~Sv}$ of NACW. The flow also makes its most rapid descent from 500 to $800 \mathrm{~m}$ in only $40 \mathrm{~km}$. We will see in section 2.6.3 that this 'rapid' descent is a result of the large frictional stresses which allow the outflow to cross $f / h$ contours. Note that section D and $\mathrm{E}$ have a bimodal structure. We saw that the two cores were most clearly distinguishable at these sections. This bimodal structure is a representation of the cores horizontal and vertical separation. We also saw that the return circulation is at mid-depth and concentrated just above the outflow (see figure 2.8b). Because this figure averages over a specific depth range some of the inflow circulation just above the lower core averages with the upper core transport. The inflow circulation can thus be seen overemphasizing the core separation and even suggesting net inflow between 520 - $620 \mathrm{~m}$ at section $\mathrm{E}$.

Finally, along the gentle descent westward of section E, the flow gradually descends from $800 \mathrm{~m}$ to $1300 \mathrm{~m}$ in $200 \mathrm{~km}$. We saw very little change in $\bar{S}$ or $S_{v w t d}$. The maximum salinity changes from greater than 37.2 pss at section $F$ to 36.57 at section $\mathrm{H}$ but shows no change for the $100 \mathrm{~km}$ further west to Cape St. Vincent. The transport increases $0.5 \mathrm{~Sv}$ between section $\mathrm{E}$ and $\mathrm{F}$, while table 2.3 listed an 
a)

Section Transport, $\quad 10^{6} \mathrm{~m}^{3} \mathrm{~s}^{-1}$
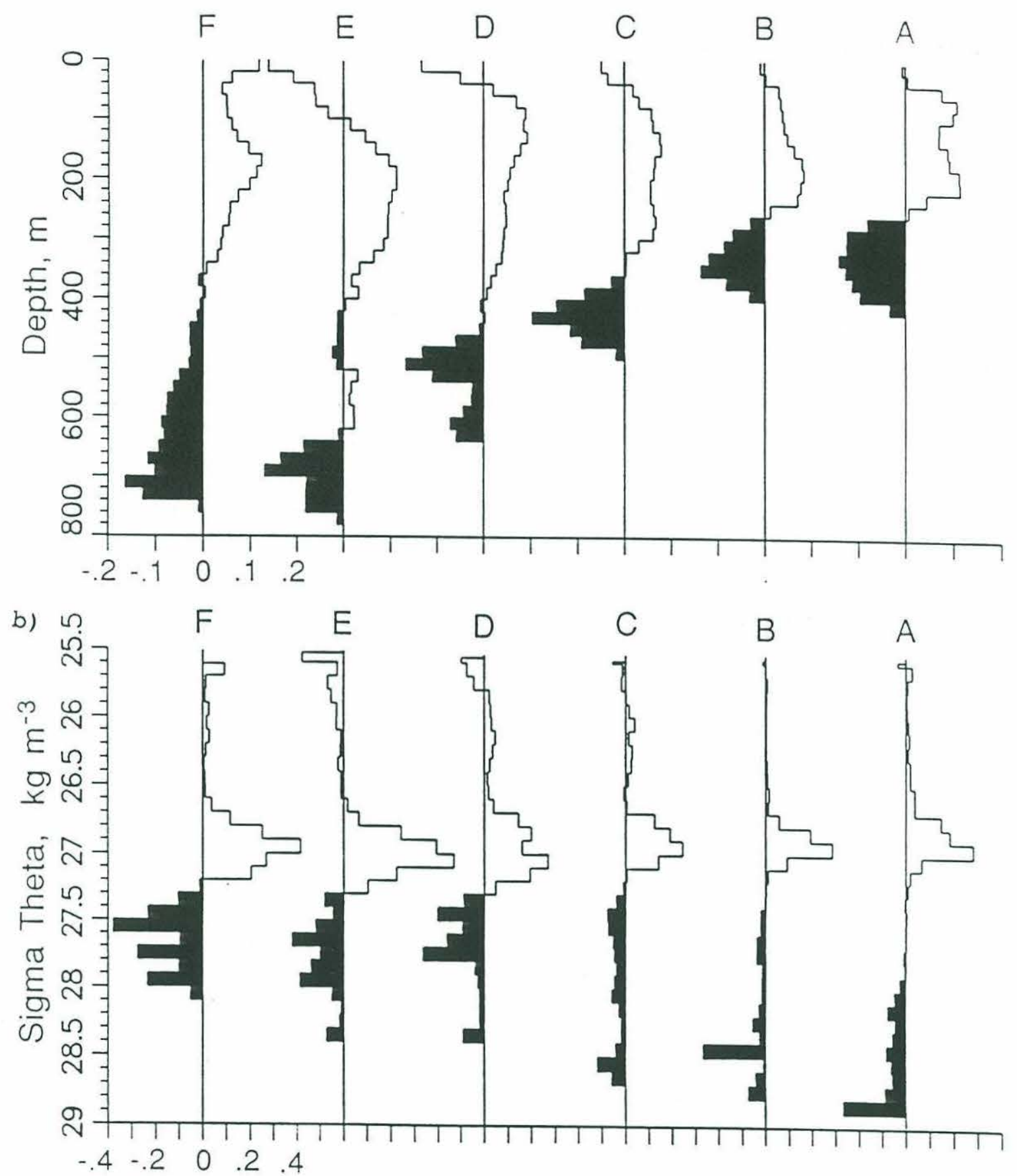

Figure 2.19: (Transport of water in $\sigma_{\theta}$ and depth classes for each section. Section $A$ is at far right. The total outflow transport is listed at the bottom of each section units are $\mathrm{Sv}$ or $10^{6} \mathrm{~m}^{3} / \mathrm{s}$. (a)transport in depth bins. (b) transport in $\sigma_{\theta}$ bins. 
increased entrainment of $0.4 \mathrm{~Sv}$ of NACW. The $\bar{S}$ indicates more pure Mediterranean water through section $\mathrm{F}$ than through sections further east which could be temporal variations in outflow transport. Clearly, there is some entrainment between sections $\mathrm{E}$ and $\mathrm{F}$, but the estimated entrainment is probably an upper bound.

\section{Transport in density classes}

Figure $2.19 \mathrm{~b}$ illustrates the transport at each station for several $\sigma_{\theta}$ water classes. At each section the transport is divided into bins of $0.1 \sigma_{\theta}$. At all sections there is no water present with density higher than $29.0 \sigma_{\theta}$ or lower than $25.0 \sigma_{\theta}$. The highest $\sigma_{\theta}$ water class is found at section $\mathrm{A}$. As the outflow moves downstream the density range narrows from $29.0-27.1 \sigma_{\theta}$ at section $\mathrm{A}$ to $28.0-27.1 \sigma_{\theta}$ at section $\mathrm{F}$ as the total transport increases. Note that at section A there is little water in the density range from $27.1-28.0 \sigma_{\theta}$, due to the highly stratified nature of the flow near the strait (see figure 2.19a) As the outflow moves downstream the highest density water mixes and disappears. There is little transport increase between sections A and C, but the internal density structure is rearranged. This pattern is consistent with a highly stratified outflow at section A internally mixing and reducing its vertical stratification without entraining fresh North Atlantic water as it moves westward. The large peaks in the transport at section A and B are probably a result of poor resolution of the flow and not 'modes' of transport.

West of section $\mathrm{C}$ the outflow begins to entrain North Atlantic water which lowers its center of gravity and lightens its potential density class. The outflow transport shifts most dramatically in the $27.3-28.0$ density classes. At section A, only $0.03 \mathrm{~Sv}$ of this water class is present but by section $\mathrm{F}$ the majority of the outflow transport, $1.3 \mathrm{~Sv}$, is in this density range. The inflow transport, which is not shaded, lies mostly in the $26.6-27.2 \sigma_{\theta}$ class. East of Section $\mathrm{F}$ the Atlantic inflow has increased considerably to feed the mixing region. Note that the North Atlantic water 
which feeds the entrainment consists of density classes just above the outflow, as expected. The density range 27.0 - 27.3 shows the largest change as the inflow moves east from section $\mathrm{F}$ to section $\mathrm{A}$. This density class is nearly absent with only 0.15 $\mathrm{Sv}$ at section $\mathrm{C}$ but $0.5 \mathrm{~Sv}$ are flowing east through section $\mathrm{D}$. This inflow water is feeding the entrainment region between section $\mathrm{C}$ and $\mathrm{D}$.

\subsubsection{Richardson Numbers}

In the previous section we established that intense entrainment occurs primarily between sections $\mathrm{C}$ and $\mathrm{D}$ and between $\mathrm{E}$ and F. Now, we consider possible mechanisms that might drive this entrainment. There has been substantial work evaluating entrainment mechanisms for surface mixed layers all of which suggest that entrainment velocities are strong functions of the Richardson number (Ellison and Turner, 1959; Price, 1979; Pollard et al., 1973). Richardson number dependent mixing has also been shown to successfully reproduce bottom boundary layer development (Thompson, 1973; MacCready and Rhines, 1993; Trowbridge, 1992). In this section, the physical justification of Richardson Number dependent entrainment will be reviewed. Then, the stability of the current is assessed through the estimations of gradient Richardson numbers and bulk Froude numbers. We find that the overflow is unstable in a gradient Richardson number sense and that stations with supercritical bulk Froude numbers are found in regions where the flow is mixing intensely.

\section{Theory}

Instability theory suggests that strongly driven currents may be susceptible to KelvinHelmholtz instability. Theoretical analysis tells us that if the available kinetic energy is strong enough to mix the stabilizing buoyancy then the current may be unstable (Turner 1973). The condition for the onset of Kelvin Helmholtz instability is formally 
set forth as a restriction on the gradient Richardson Number, Ri defined as

$$
R i_{g}=\frac{g}{\rho_{o}} \frac{\partial \rho}{\partial z} /\left|\frac{\partial \vec{u}}{\partial z}\right|^{2}
$$

and when $R i_{g}<R i_{\text {critical }}$ the current may be unstable. For most purposes $R i_{\text {critical }}$ is $\frac{1}{4}$. This critical Richardson number is extremely sensitive to the density profile as a whole and for particular profiles can be lower (Turner 1973). For instance, the critical Richardson number value may be lowered by the presence of horizontal boundaries (Hazel 1972). There is also a instability with $R i_{g}>\frac{1}{4}$ for the special case in which two interfaces close to each other become unstable when waves that move at the same speed on both interfaces interact (Taylor 1931; Goldstein 1931; Miles and Howard

1964). In general, however, $R i_{g} \geq \frac{1}{4}$ is a sufficient condition for the flow to be stable.

\section{Indications of overturning in the data}

There are several indications that Kelvin-Helmholtz instability may be the mechanism that drives the mixing and not bottom generated turbulence. Most profiles have the maximum velocity off the bottom which then decreases upwards towards the interface (figure $2.15 \mathrm{a}$ and $2.15 \mathrm{~b}$ ). Most of the profiles are also weakly stratified through the lower portion is the outflow and highly stratified at the interface with NACW, suggesting the separation of the outflow into two layers: a bottom boundary layer influenced by bottom friction and an interfacial layer formed through entrainment and mixing of NACW. Rolf Lueck's (1989) dissipation measurements indicate a minimum in dissipation at the same depth as the velocity maximum suggesting that bottom turbulence is not a leading contributor to mixing at the interface (Johnson et al., 1993). bottom generated turbulence also tends to form a well mixed profile in density. but most profiles are strongly stratified above the $10 \mathrm{~m}$ closest to the bottom.

Thorpe (1971) has shown that Kelvin Helmholtz instability can produce intermediate layering of the density profile. Figure 2.20, from Woods and Wiley (1972), 
shows a schematic of the intermediate layering that can occur in Kelvin-Helmholtz instability. One smooth gradient, of depth $2 \mathrm{~h}$, between two layers develops into two gradients, each with thickness $h$, or three layers. They also show that the intense mixing as the billow overturns is intermittent in space and time. Only $\frac{2}{5}$ of the surface area of the sheet is involved in active turbulence and a time averaged critical Ri for the observed overturning is $\frac{3}{4}$. A majority of the sections exhibit some degree of layering of the stratification within the outflow (Figure 2.15(c)).

Repeat occupations of station 75 demonstrate the intermittent structure of the outflow and the large variability in the dissipation measurements. A total of four repeat CTD casts were made at station 75 , six repeat XCP profiles, and six XDP profiles. Figure 2.21 shows two occupations of station 75 with the CTD, XCP and XDP profiles. The velocity profile is remarkably steady with a standard deviation of about $10 \mathrm{~cm} / \mathrm{sec}$. The XDP profiles both show large dissipation values in the outflow layer with a relative minimum close to the velocity maximum (Lynch and Lueck). The variability in the dissipation measurements is quite large, with an order of magnitude difference in average peak values in the outflow layer $\left(10^{-1}\right.$ vs. $\left.10^{-2}\right)$. Other dissipation profiles at this station generally fell within this range. The CTD casts show large differences in the salinity: the maximum salinity is 38.2 vs. 37.9 for the separate casts. Note that to some degree these differences reflect the spatial differences in horizontal structure because the repeat casts are not precisely coincident. In fact, the two repeat occupations of station 75 shown here were $.6 \mathrm{~km}$ apart. These repeat casts also reflect the temporal variability of the flow. One station exhibits layering in both temperature and salinity while the other has a smooth gradient in properties through the same depth. This intermittent layering observed is consistent with Woods and Wiley's schematic of Kelvin-Helmholtz billowing. The mixing appears to vary spatially and temporarily which is consistent with Kelvin-Helmholtz instability (but not bottom generated turbulence). 

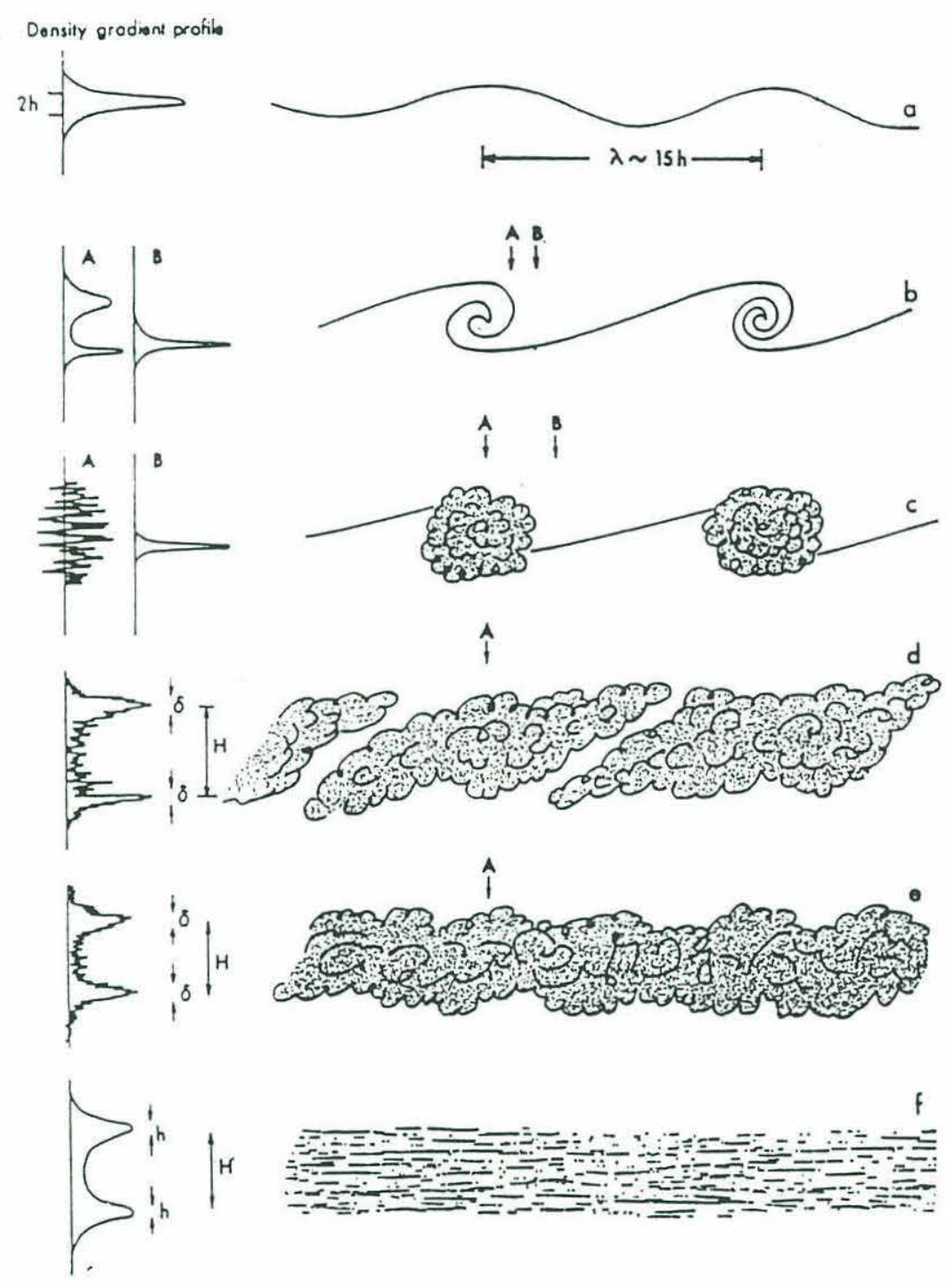

Figure 2.20: Schematic showing the development of an interface through KelvinHelmholtz instability. One density gradient becomes two gradients separated by a weakly stratified layer as an intermediate result of the instability. 


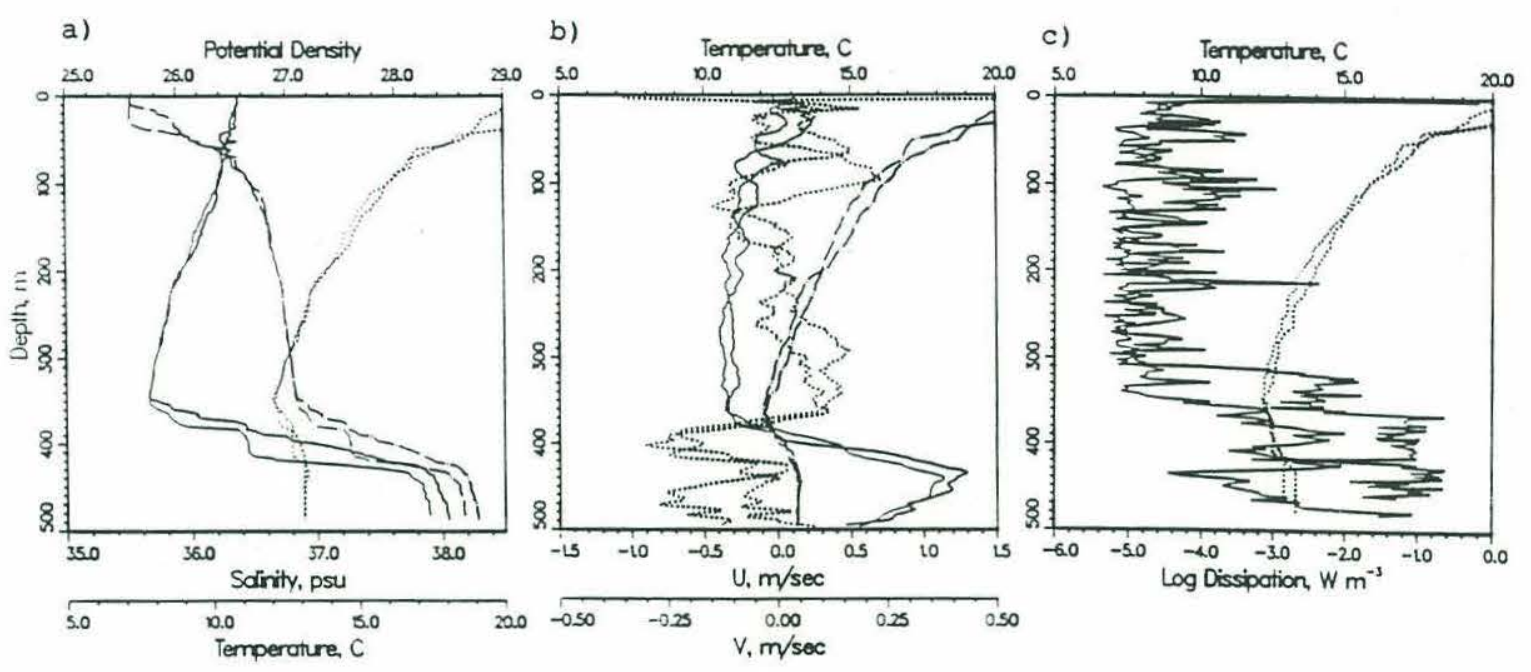

Figure 2.21: Repeat occupations of station 75 show the variability in the outflow. (a). CTD 75 and CTD 91 salinity (solid line), temperature (dashed line), potential density (chained line). (b). XCP 2544 and XCP 2556 projected into the direction of the maximum velocity. $\mathrm{u}$ is the solid line, $\mathrm{v}$ is the dashed line and the temperature from the XCP probe is the chained line. (c). XDP 704 and 804 dissipation (solid line) and temperature (dashed line). 
We also observe vertical overturning of unstable density profiles. For instance, CTD station 86 in section D (figure 2.15c) has an unstable density gradient that is reminiscent of a Kelvin Helmholtz billow where salty outflow water is rolling up relatively fresh water (Figure 2.15d).

\section{Observations of gradient Richardson numbers}

To explore the possible instability of the outflow, the $R i_{g}$ as defined above was calculated using CTD/XCP drop pairs which are defined as profiles that were taken within 30 minutes and 1 nautical mile of each other (see Kennelly et al 1989). A least square fit over $20 \mathrm{~m}$ was used to determine the vertical gradients, namely $\partial \rho / \partial z$ and $\partial \vec{u} / \partial z$ (Millard et al., 1990), i.e. a steric leveling as with $N^{2}$ calculations). These $R i_{g}$ calculations are fairly sensitive to the length scale over which the vertical gradients are computed. Because of the sensitivity of these calculations, speculations based on the specific value of the Richardson numbers are suspect and instead only relative values of gradient Richardson numbers are compared. Only a qualitative picture of the relative stability of the currents can be obtained by noting the location of a profiles lowest $R i_{g}$ and only stations with low $R i_{g}$ are considered as candidates for the onset of instability.

Typical gradient Richardson number profiles are illustrated in Figure 2.15(a) with the three dimensional velocity profile for CTD station 65 in section A (near $11 \mathrm{~km}$ in Figure 2.3(b)). Here the outflow is confined to below $200 \mathrm{db}$ moving to the SW with maximum speed of $67.4 \mathrm{~cm} / \mathrm{s}$. Low $R i_{g}$ are found near the interface at 225 and $250 \mathrm{db}$ and at the bottom of the profile where the there is a well mixed layer. Notice that this profile has the classical 'nose' shape of a boundary layer current with the maximum velocity $50 \mathrm{db}$ above the bottom. The velocity also rotates through the whole depth of the outflow in the same sense as an Ekman spiral. This profile does not actually have $R i_{g}<\frac{1}{4}$, but does contain values below $\frac{3}{4}$ at the interface. CTD 
station 76 (Figure 2.15b), on the other hand, contains much lower $R i_{g}$ regions, with values well below $\frac{1}{4}$. Here the outflow moves NW with the maximum velocity within $20 \mathrm{~m}$ of the bottom. Very low $R i_{g}$ are again found near the bottom of the profile and near the interface between the Mediterranean outflow and the overlying fresh North Atlantic return circulation. Notice that this velocity profile spirals with depth in the classical Ekman layer sense above the velocity maximum, as does the velocity at CTD 65. However, below the velocity maximum the current veers to the north in the opposite direction of a bottom Ekman spiral. In general, the bottom of the CTD profiles contain a well mixed or only weakly stratified layer that is dominated by bottom stress. Any velocity shear generated by the bottom stress would indicate low $R i_{g}$ and would not be a valid region of Kelvin-Helmholtz instability. At the interface, however, Kelvin-Helmholtz instability is a possible mechanism for the intense mixing and dilution of the overflow.

\section{Observations of bulk Froude numbers}

The $R i_{g}$ calculations have shown that the flow is potentially unstable at the interface between Mediterranean outflow and NACW even though a large density gradient stabilizes the flow. $R i_{g}$ numbers are difficult to compare station to station because the structure of the profile must be considered. We now examine using a single number for each station, the bulk Froude number, $F r_{b}$, to characterize the stability of the outflow. Layered models of entrainment have suggested that the appropriate stability parameter is the bulk Froude number (Turner 1973). The Bulk Froude numbers as defined by

$$
F r_{b}^{2}=\frac{\rho_{o} \delta U^{2}}{g H \delta \rho},
$$

where $\delta U$ is the velocity difference between layers, $\delta \rho$ is the density difference, and $\mathrm{H}$ is the thickness of the lowest layer. When $F r_{b}>1$ the layer may be unstable. Actually, laboratory models of entrainment experiments, suggest that the appropriate 
instability criterion is for the bulk Richardson number, $R i_{b}<0.65$ (Price, 1979), where $R i_{b}=F r_{b}^{-2}$. This is equivalent to $F r_{b}>1.2$. So, we expect the flow to begin entraining as the bulk Froude number raises above one with more increasingly vigorous entrainment as $F_{r_{b}}$ becomes larger (see chapter 3 for a discussion on entrainment parameterizations). Each CTD/XCP station pair can be assigned one $F r_{b}$. Thus $F r_{b}$ numbers are much simpler use than $R i_{g}$ to determine the spatial distribution of unstable profiles.

\section{Calculating $F r_{b}$}

To determine $F r_{b}$, theoretical models have assigned $\delta \rho$ and $\delta U$ as the differences between the extreme values within the two layers: i.e. $\delta \rho=\rho_{2}-\rho_{1}$ where $\rho_{1}$ and $\rho_{2}$ are the upper and lower layer densities. Theoretical models thus ignore any gradient region between the two layers (at least to determine the density). In this data however, the velocity and density are not well mixed so some layered approximation must be used. The average velocity of the outflow is calculated. The inflow layer is assumed to be above the T,S minimum (note that this is above the zero velocity depth when no net salt flux is required). Vertical averages above the T,S minimum over a $30 \mathrm{~m}$ interval define the inflow velocity and density. Note that the density is calculated as potential density referenced to the depth of the T,S minimum. The outflow density is velocity weighted density, similar to the velocity weighted salinity defined in equation $2.1 . \delta \rho$ and $\delta U$ are the difference between the the average density and velocity of the outflow and inflow layers. For instance, the $F r_{b}$ calculated in this standard way for station CTD 76 is 1.2 (Figure 2.15(b)).

Like the $R i_{g}$ number, the $F r_{b}$ number is sensitive to the calculation of $\delta \rho$ and $\delta U$. To understand the range of reasonable $F r_{b}$ (this range does not represent error bars), upper and lower bounds on $F r_{b}$ are calculated along with the standard value. The upper bound on $F r_{b}$ is obtained by calculating $\delta U$ using the difference between 


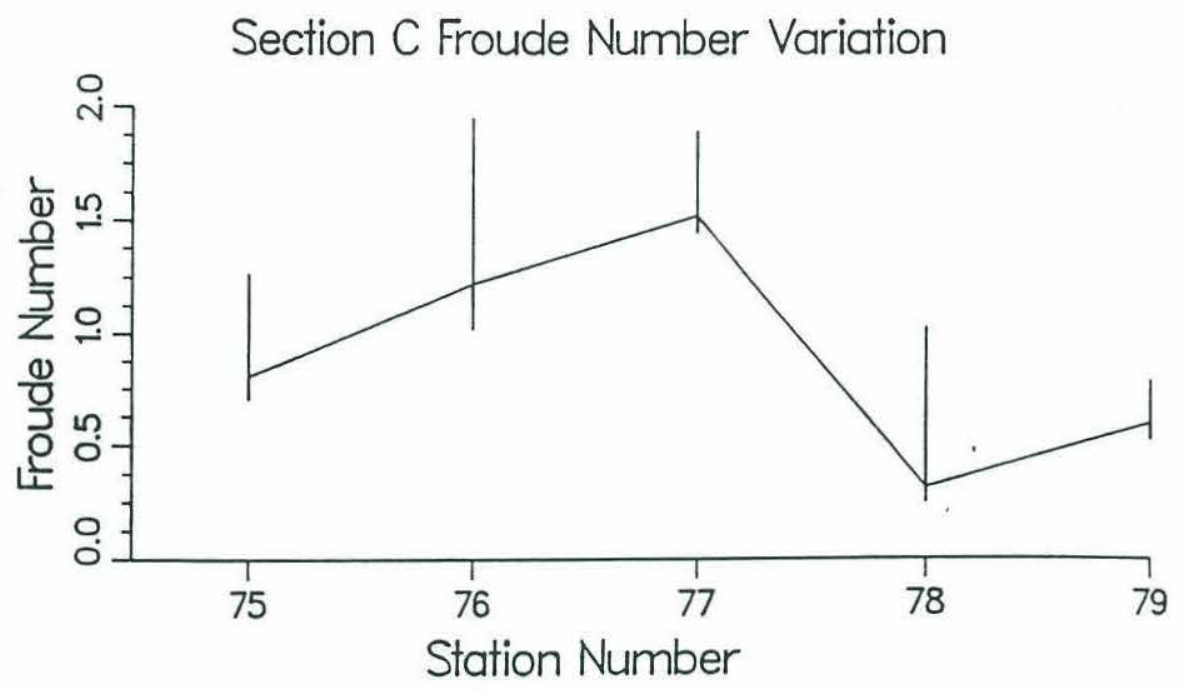

Figure 2.22: Variation of Bulk Froude numbers across section $\mathrm{C}$ (solia iine). At section $\mathrm{C}$ where the current undergoes an inertial turn (see chapter 2.6.2) onto the continental slope, the bulk Froude number suggests supercritical values near the axis of the flow. Vertical error bars represent possible range of Froude number values depending upon details of the calculation. High Froude numbers were calculated when the maximum outflow velocity is used to estimate the shear (dotted line). Low values were calculated when the maximum outflow density is used to estimate the stabilizing density gradient (dashed line).

the maximum outflow speed and the entrained water speed (i.e. inflow velocity) rather than the average outflow velocity. To obtain a lower bound, $\delta \rho$ is estimated using the maximum density difference instead of layer averages. Note that these bounds are consistent with the way theoretical models calculate $F r_{b}$ (Ellison and Turner, 1959) since these bounds also collapse the region of large property gradients between the layers into an infinitely thin layer. The $F r_{b}$ varies considerably within this range, as well as, across individual sections (Figure 2.22 examines section $\mathrm{C}$ as an example). At section $C$ supercritical values of $F r_{b}$ were found at the axis of the current where velocity and salinity were at a maximum. The range in possible $F r_{b}$ is large but certainly for CTD 76, the current is supercritical. 
$F r_{b}$ map

Each station is assigned a $F r_{b}$ and shown in figure 2.23. Supercritical values of $F r_{b}$ occur in the Strait of Gibraltar and at section C. Layered hydraulic control theory also says the $F r_{b}$ must equal unity at the Camarinal sill for critical control (Whitehead et al., 1974), which we find. Note that this critical control is consistent with the observed interface height deviations along the strait (see figure 2.6). Table 2.3 suggests some entrainment occurs in the Strait coincident with the large $F r_{b}$ there. Previously, we saw that most of the entrainment takes place between section $\mathrm{C}$ and D and then further between section $\mathrm{E}$ and $\mathrm{F}$ (figure 2.18 and table 2.3). The entrainment at section $\mathrm{C}$ coincides with large $F r_{b}$. The entrainment between sections $\mathrm{E}$ and $\mathrm{F}$ also coincides with supercritical $F r_{b}$. Large $F r_{b}$ values very near one are found near stations 92 and 96, coincident with the upper and lower cores of the flow (see figure 2.11).

The two individual cores of outflow also entrain NACW at different locations downstream of the strait (also see Section 2.4). Between section $\mathrm{E}$ and section $\mathrm{F}$ the upper core increases substantially in transport. Assuming the upper core at section E is sampled by stations northward of $30 \mathrm{~km}$ and the upper core at section $\mathrm{F}$ includes stations above $38 \mathrm{~km}$ the transports of the upper and lower cores at each section can be calculated (see the XCP velocity sections in figure $2.8 \mathrm{~b}-\mathrm{c}$ ). The upper core transport more than doubles its outflow transport from $0.39 \mathrm{~Sv}$ to $0.95 \mathrm{~Sv}$ between sections $\mathrm{E}$ and $\mathrm{F}$. The lower core, however, does not gain any volume flux but primarily splits into two branches, the smaller branch containing only $0.13 \mathrm{~Sv}$ moving through Section FE. The geostrophic velocity estimates also confirm the doubling of the upper core transport and the lower core branching. Both the geostrophic velocities and the XCP velocities indicate that the predominant entrainment between Sections E and $\mathrm{F}$ occurs in the upper core. The critical $F r_{b}$ at section $\mathrm{E}$ is located at the upper core, coincident with the impending entrainment there. Note that at section $\mathrm{F}$ 


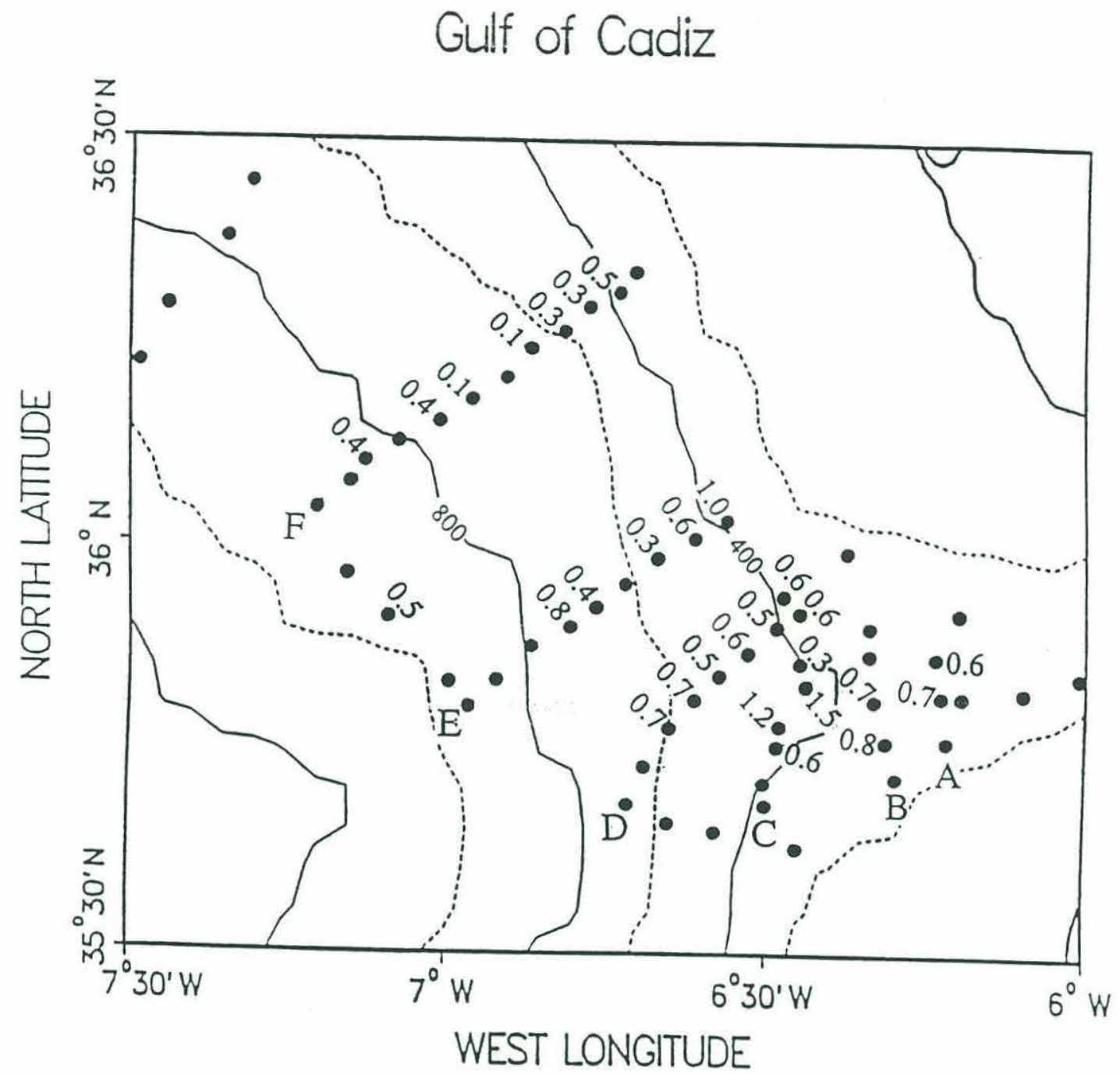

Figure 2.23: Station map listing selected Bulk Froude number estimates. Near the strait the Bulk Froude numbers were higher than at Section F where less mixing is taking place.

(Figure 2.3(d)) the continental slope near the upper core increases and the depth of the upper core increases from $514 \mathrm{db}$ at section $\mathrm{E}$ to $672 \mathrm{db}$ at section $\mathrm{F}$ (table 2.2), suggesting acceleration and ageostrophic effects which would allow the upper core to cross isobaths. 


\section{Summary}

Figure 2.23 confirms that supercritical values of $F r_{b}$ occur near regions of intense entrainment, between section $\mathrm{C}$ and $\mathrm{D}$, in the upper core between section $\mathrm{E}$ and $\mathrm{F}$, and in the Strait of Gibraltar. Low values of $R i_{g}$ were found at the interface between westward flowing Mediterranean water and eastward flowing NACW above. The outflow is susceptible to Kelvin-Helmholtz instability and bulk Froude numbers are good indicators of active entrainment. 


\subsection{Dynamics}

The dynamics of the outflow are considered here by examination of the changes on momentum and mass of the outflow. To simplify the analysis we will consider the average properties of the flow by defining a coordinate system that follows the flow and integrate across a cross section of the flow. Batchelor (1967) defines a streamtube as "the surface formed instantaneously by all the streamlines that pass through a given closed curve in the fluid." The closed curve defining the Mediterranean outflow stream-tube is the interface between the salty Mediterranean water and the NACW above it. The along-stream coordinate follows the streamlines downstream (like the core salinity section in figure 2.6). The cross-stream coordinate is perpendicular to the mean axis of the flow and normal to sections A-H.

First, the derivation of the integrated momentum and continuity equations is reviewed. Then, the cross stream momentum equation is examined to see if the along-stream component of velocity is in geostrophic balance. Next, the along-stream momentum equation is examined to evaluate the importance of friction in the descent of the overflow. Then, the buoyancy flux into the outflow is quantified by considering the downstream changes of mass and salinity. The entrainment rate is written as an interfacial stress and compared with the total stress estimated from the along-stream momentum equation. We find that the flow undergoes geostrophic adjustment where nonlinearity becomes important and very large stresses retard the flow.

\subsubsection{Integrated Momentum and Continuity Equations}

Before we can examine the effect and relative importance of the Coriolis force, friction, and entrainment on the evolution of the Mediterranean outflow, we need to derive the integrated momentum equations. Smith (1973) gives a thorough derivation 
of the stream-tube equations which we follow with only subtle variations. An outline of this derivation follows.

Curvilinear coordinates are used, which follow the flow and capture the essential dynamics better than regular Cartesian coordinates (see Batchelor 1967). For the stream-wise coordinates to be unique and well defined, the current is assumed to be narrower than the local radius of curvature, ie. quasi-parallel (Note that unique stream-wise coordinates could not be defined for a radially symmetric flow because the downstream coordinate would not be well defined). The along-stream and crossstream coordinates, $\zeta$ and $\eta$, have corresponding velocities $u$ and $v$. $\beta$ is the angle the along-stream coordinate makes with the $\mathrm{x}$ axis. ${ }^{7}$

The current is assumed to be steady and confined to a broad thin layer entirely in contact with the continental slope. The bottom slope is assumed to be small (i.e. the slope, $\alpha^{2} \ll R_{o}^{-1}$, where $R_{o}$ is the Rossby number) and the Rossby number is less than one. The combination of these two assumptions assures that the pressure is in hydrostatic balance. The along-stream velocity, $\mathrm{u}$, is assumed much larger than the cross-stream velocity, $\mathrm{v}$ and the properties are assumed to change downstream much faster than across stream. ${ }^{8} 9$

In this derivation, the current is not assumed to be uniform in cross section. The outflow velocity is assumed to go to zero along some boundary, $z=-D+h$, where $\mathrm{h}$ is the height of the outflow and the bottom depth is given by $z=-D(\zeta, \eta)$. The width of the outflow is well defined by the boundary $\eta= \pm l$. The equations of motion are integrated over the depth and width of the outflow, to the boundary where $u=0$ (so that we may simplify the pressure gradient). Using all these assumptions

\footnotetext{
${ }^{7}$ In this derivation the $\mathrm{z}$ coordinate remains in the direction of gravity: i.e. the curvilinear coordinates are not aligned normal to the bottom slope (as with Smith (1973)).

${ }^{8}$ Note this is less strict than Smith's assumption that the flow is uniform in cross section.

${ }^{9} U \gg V$ and $L_{\zeta} \gg L_{\eta}$ removes the nonlinear terms and the metric terms arising from the coordinate transformation.
} 
the integrated momentum equations are

$$
\begin{aligned}
\frac{\partial}{\partial \zeta}\left(\iint_{A} \rho u^{2} d z d \eta\right) & =-\iint_{A} \frac{\partial p}{\partial \zeta} d z d \eta-\int_{-l}^{+l}\left(\tau_{B}+\tau_{I}\right) d \eta . \\
\iint_{A} \rho_{o} u\left(f+u \frac{\partial \beta}{\partial \zeta}\right) d z d \eta & =\iint_{A} \frac{\partial p}{\partial \eta} d z d \eta
\end{aligned}
$$

$\tau_{B}$ and $\tau_{I}$ are the bottom stress and interfacial stress respectively. The integrated (or stream-tube) continuity equation is

$$
\frac{\partial}{\partial \zeta}\left(\iint_{A} u d z d \eta\right)=\int_{-l}^{+l} w_{*} d \eta
$$

The continuity equation says that the downstream divergence of the outflow transport is balanced by entrainment. The cross-stream momentum equation, equation 2.9, suggests that the along-stream velocity is approximately in geostrophic balance modulated by the curvature of the path. In polar coordinates this is just the familiar cyclostrophic balance. The cross stream pressure gradient is partially balanced by $U^{2} / R$, where $\mathrm{R}$ is the radius of curvature. The along-stream coordinate momentum equation, equation 2.8, says that the total Bernouilli function or momentum flux changes downstream due to the total stress acting on the flow.

These equations are essentially the same as Smith (1975) except no approximations assuming a thin jet have been used to simplify the pressure gradient term. Here, the pressure term is calculated without approximation. Also, the exterior circulation is not motionless as Smith assumes in his reduced gravity formulation. Smith also needlessly eliminates some terms which disappear though the integration process if you assume that horizontal entrainment is negligible and that the boundaries of the flow are stream lines. For example, when integrating the continuity equation, if the edges of the flow, $\pm l$, are streamlines then

$$
\int_{-l}^{+l} \partial_{\eta} v d \eta=\left.u \partial_{\zeta} l\right|_{-l} ^{+l} .
$$

Also from the continuity equation 2.10 , applying the Leibnitz rule,

$$
\int_{-l}^{+l} \partial_{\zeta} u d \eta=\partial_{\zeta} \int_{-l}^{+l} u d \eta-\left.u \partial_{\zeta} l\right|_{-l} ^{+l} .
$$


The terms $\left.u \partial_{\zeta} l\right|_{-l} ^{+l}$ will cancel with no further approximation necessary. Note that the boundary terms from the vertical integral will only partly cancel along $z=-D+h$, which is not a streamline because of the entrainment velocity, $w_{*}$ across this interface.

\subsubsection{Cross-stream momentum}

The Mediterranean outflow is very dense water that encounters a sloping bottom after exiting the Strait of Gibraltar. In the absence of friction, the outflow would accelerate down the slope into deep water driven by the pressure gradient. This downslope pressure gradient is balanced by the Coriolis force. Thus the effect of rotation is to limit the downslope excursion of the flow as geostrophic balance is achieved. Thus the outflow could undergo a geostrophic adjustment as the flow exits the Strait and first encounters the continental slope. The outflow would accelerate down the slope which would increase the coriolis force. When the Coriolis force becomes large enough to balance the pressure gradient the downslope acceleration would cease and the outflow would follow $\mathrm{f} / \mathrm{h}$ contours (in the absence of friction).

In the Gulf of Cadiz Expedition the outflow exits the Strait of Gibraltar and flows along the continental shelf confined to a channel between sections $\mathrm{A}$ and $\mathrm{B}$ (note that the average bottom depth across these sections is similar, see figure 2.3(a) and A.3). After section B the outflow reaches the edge of the continental shelf and encounters the continental slope with isobaths oriented in the nearly north/south direction, perpendicular to the flow direction (Figure 1.7). In figure 2.4, the current makes a sharp right hand turn downstream of section $\mathrm{B}$ and becomes aligned along isobaths downstream of section $\mathrm{D}$.

Ochoa and Bray (1991) suggest that this northward deflection of the outflow is forced by the presence of a north/south ridge steering the current (Figure 1.7) but Kenyon and Belderson (1973) suggests that the ridge is depositional and is likely 
caused by the persistence of the outflow over decades. We will show that the flow is undergoing geostrophic adjustment and the deflection of the current is an inertial turn, supporting the second view that the ridge has been deposited and the channel has been carved into the bottom by the strong currents of the outflow.

We saw in the cross-stream momentum equation (equation 2.9), that the geostrophic balance of the along-stream velocity is modified by the curvature of the flow. This can approximately be written as

$$
f V+V^{2} \frac{d \beta}{d \zeta} \approx-\frac{1}{\rho_{o}} \frac{\partial p}{\partial \eta}
$$

where $\beta$ is the angle the trajectory makes relative to the along-stream coordinate, $\zeta$. The relative influence of the trajectory curvature (the second term on the left hand side of the cyclostrophic equation above) can be assessed by considering the Rossby number defined as the ratio of the Coriolis force to the curvature, $R_{c}=$ $U \beta / f L$, where $L$ is the downstream length scale (i.e. the scale for $\zeta$ ). The average change in direction of the outflow between section $B$ and $D$ is $86^{\circ}$. This is a very large curvature of the flow path (or trajectory). Near section $\mathrm{C}, U=0.64 \mathrm{~m} / \mathrm{s}$, $L=24.4 \mathrm{~km}, f=8.5 \times 10^{-5} \mathrm{~s}^{-1}$, and $\beta=1.5$ radians. Then the curvature Rossby number, $R_{c}=\frac{1}{2}$. Inertial effects are therefore very important where the outflow makes its initial descent. The geostrophic velocity estimates for section $\mathrm{C}$ confirm the importance of the flow curvature in balancing the Coriolis force. At section $\mathrm{C}$ the geostrophic velocities severely underestimate the observed XCP velocities. The maximum geostrophic velocity at section $C$ was less than $-30 \mathrm{~cm} / \mathrm{sec}$ although the $\mathrm{XCP}$ velocity at station 75 in section $\mathrm{C}$ is $-135 \mathrm{~cm} / \mathrm{sec}$. The potential density field also suggests very small velocities at section $\mathrm{C}$ (figure $2.5(\mathrm{~b})$ ). The reference level of zero velocity lies near $\sigma=27.1$. The isopycnals below this reference isopycnal are nearly flat, suggesting little shear in velocity. Also, the current is restricted between two channels in the topography and due to the poor station spacing the slope of 
isopycnals in the core of the outflow cannot be determined (i.e. the bottom triangle problem), which could contribute to the low geostrophic velocities there. ${ }^{10}$

\section{Geostrophic adjustment}

To consider the implications of the strong influence of rotation on the flow we will assume that the outflow undergoes frictionless geostrophic adjustment. Following Griffiths (1986), we calculate the descent of a density driven current into a homogeneous ocean due to geostrophic adjustment in the absence of friction or entrainment assuming that the along stream velocity is geostrophic and has uniform zero potential vorticity. For a density driven current exiting a broad flat channel onto an inclined plane given by $z=-\alpha y$ (figure 2.24), geostrophy on the plane is given by

$$
f\left(u-U_{\alpha}\right)=-g^{\prime} \partial h / \partial y
$$

where $g^{\prime}$ is reduced gravity and $U_{\alpha}$ is the mean geostrophic velocity along the slope, $g^{\prime} \alpha / f$. Conservation of potential vorticity gives us

$$
(f+\xi)=f / H_{o},
$$

where $\xi$ is the vorticity and $H_{o}$ is the equivalent depth. Equations 2.6 .2 and 2.6.2 can be combined to form a second order ordinary differential equation for the height of the fluid, $h$.

If we assume that the potential vorticity is zero in the channel where the fluid is on a flat bottom supported by channel walls and $v(x=0, y=0)=0$, the flow satisfies

$$
v=-f x
$$

and

$$
h=h_{o}\left(1-\frac{1}{2} x^{2} / \lambda^{2}\right),
$$

\footnotetext{
${ }^{10} \mathrm{Geostrophic}$ velocities were assumed constant below the deepest common level.
} 


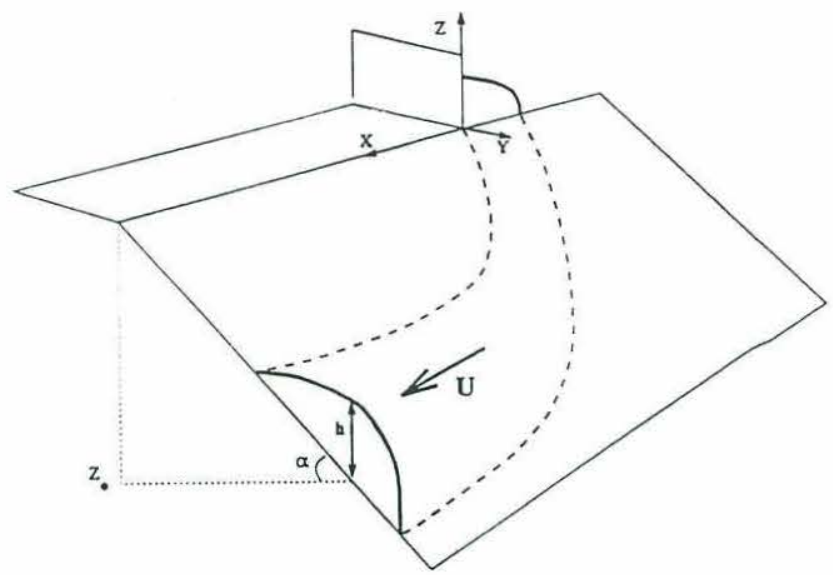

Figure 2.24: Idealized schematic of a potential vorticity conserving outflow after Griffiths (19XX). The outflow begins at the origin confined to a broad flat channel and descends along a constant slope topography. Far downstream, the center of the outflow is located at $\left(y_{*}, z_{*}\right)$, has height at the center of $h_{*}$ and half width $l$.

where $\lambda$ is the Rossby radius, $\left(g^{\prime} h_{\circ}\right)^{\frac{1}{2}} / f$ and $h_{\circ}$ is the height of the fluid along the channel wall at $x=0$. Somewhere far downstream, after the layer has adjusted along the slope, the flow satisfies

$$
u=U_{\alpha}+f\left(y-y_{*}\right)
$$

and

$$
h=h_{*}-\frac{1}{2} h_{\circ}\left(\frac{y-y_{*}}{\lambda}\right)^{2},
$$

where $\left(y_{*}, z_{*}\right)$ define the location of the center streamline of the flow (see figure 2.24).

The unknown height of the center streamline, $h_{*}$, can be determined by conserving volume flux between the channel and the downstream position. The unknown position on the slope, $y_{*}$, can be determined by applying the Bernoulli equation to the lowest, deepest streamline defined by the subscript 1 . The Bernouilli equation applied to the lower streamline is

$$
g^{\prime} z_{1}+\frac{1}{2} u_{1}^{2}=\frac{1}{2} v_{1}^{2}(y=0)
$$


which determines $z_{1}$. The center streamline, $z_{*}$, is given by $z_{1}+l \alpha$, where $l$ is the half width of the flow. The centerline of the current, $z_{*}$, is therefore given by

$$
z_{*}=l \alpha+h_{o}\left[1-1 / 2\left[F+(4 / 3 F)^{-1 / 3}\right]^{2}\right]
$$

where $F=\left(g^{\prime} \alpha / f\right) /\left(g^{\prime} h_{\circ}\right)^{1 / 2}$ and $l=\left(g^{\prime} h_{\circ}\right)^{1 / 2} / f \cdot(4 / 3 F)^{-1 / 3}$. The parameter $h_{\circ}$ can be found in terms of the total outflow volume transport, $Q$, from

$$
h_{o}=\left(2 Q f / g^{\prime}\right)^{1 / 2} .
$$

For the Mediterranean outflow with slope $\alpha=10^{-2}, g^{\prime}=1.1 \cdot 10^{-2} \mathrm{~m} / \mathrm{s}^{2}$, and $Q=0.7 \mathrm{~Sv}$, this purely inertial descent would be only $10 \mathrm{~m} \cdot{ }^{11}$ According to the hydrographic sections however, the center of mass of the current descends over $160 \mathrm{~m}$ between section B and D while the lowest streamline descends more than 250 m. In fact, the outflow descends between $500-1000 \mathrm{~m}$ before it becomes neutrally buoyant and is 'fully adjusted'. It seems that inviscid geostrophic adjustment alone is insufficient to account for the steep descent of the Mediterranean outflow down the continental slope. Frictional forces must be present to break the geostrophic constraint that the current to follow isobaths ( $f / h$ contours).

\subsubsection{Along-stream Momentum Equation}

In the previous section we found that the Mediterranean outflow would rapidly geostrophically adjust upon exiting the Strait. However, the initial descent of the outflow is much greater than simple geostrophic adjustment would suggest. Some other process must allow the flow to cross $f / h$ contours. The along-stream momentum equation can be examined to diagnose the magnitude of the friction required to force the Mediterranean outflow down the continental slope into the Gulf of Cadiz.

\footnotetext{
${ }^{11}$ Note that the lowest streamline descends almost $120 \mathrm{~m}$.
} 


\section{Evaluating the pressure gradient}

The along-stream pressure gradient term, as shown in equation 2.8 , can be rewritten by assuming that the pressure is hydrostatic using the usual decomposition of the density field into a homogeneous portion $\rho_{o}$ and its perturbation, $\rho^{\prime}$.

These equations are essentially the same as those of Smith (1975) except that the thin jet approximation has not been used to simplify the pressure gradient term. Instead, to simplify the pressure gradient term the hydrostatic balance is used and the current is integrated to the level of no motion. The pressure in the outflow is given by

$$
p(z)=p(-D+h)+\int_{z}^{-D+h}-g \rho^{\prime} d z^{\prime}
$$

Since the interface $z=-D+h$ is a level of no motion, $\partial_{\zeta} p(-D+h)=0$. Therefore,

$$
\iint \partial_{\zeta} p d \eta d z=\iint_{-D}^{-D+h} \partial_{\zeta}\left(\int_{z}^{-D+h} g \rho^{\prime} d z^{\prime}\right) d z d \eta
$$

Successive applications of Leibnitz's rule coupled with the assumption that the current is bounded by regions where $u=0$ and $\rho^{\prime}=0$ yield the final expression for the pressure gradient,

$$
-\iint_{A} \frac{\partial p}{\partial \zeta} d z d \eta=-\frac{\partial}{\partial \zeta} \iint_{-D}^{-D+h} \int_{z}^{-D+h} g \rho^{\prime} d z^{\prime} d z d \eta+\iint_{-D}^{-D+h} g \rho^{\prime} d z \partial_{\zeta} D d \eta
$$

The first term on the right hand side of equation 2.12 is the internal pressure gradient term because it expresses the changes in potential energy stored in a column of height, h. The second term is the external pressure gradient term because it represents the gravitational force on a whole fluid column sitting on a slope. Smith neglects this internal pressure gradient term in his streamtube model. We find that this 'internal' pressure gradient term is critically important where the outflow is still confined close to the Strait along a flat shelf, but is of less importance further downstream. 
The full along-stream momentum equation is then,

$$
\begin{array}{r}
\frac{\partial}{\partial \zeta}\left(\iint_{A} \rho u^{2} d z d \eta\right)+\frac{\partial}{\partial \zeta} \iint_{-D}^{-D+h} \int_{z}^{-D+h} g \rho^{\prime} d z^{\prime} d z d \eta \\
-\iint_{-D}^{-D+h} g \rho^{\prime} d z \partial_{\zeta} D d \eta,=-\int_{-l}^{+l}\left(\tau_{B}+\tau_{I}\right) d \eta
\end{array}
$$

The first and second terms on the left hand side are simple to calculate from the XCP and CTD data at each section. Calculating the third term on the left hand side is more problematic because it is difficult to define streamlines within the flow to calculate $\partial_{\zeta} D$. Instead, we assume

$$
\iint_{-D}^{-D+h} g \rho^{\prime} d z \partial_{\zeta} D d \eta \approx \partial_{\zeta} D^{*} \iint g \rho^{\prime} d z d \eta
$$

where $D^{*}$ is defined by

$$
D^{*}=\frac{\iint g \rho^{\prime} D d z d \eta}{\iint g \rho^{\prime} d z d \eta} .
$$

Equation 2.14 means that the along-stream change in bottom depth is assumed uniform across each section and is well represented by the depth of the center of mass of the outflow.

We must also define how to calculate the downstream derivative, $\partial_{\zeta}$, of the kinetic energy term and the internal potential energy term. Here we define the downstream distance as the distance from the center of each section. The center of each section, where $\eta=0$, is defined as the center of density anomaly. ${ }^{12}$ Namely, the center of the outflow, $X$, is given by

$$
X=\frac{\iint \rho^{\prime} \eta d z d \eta}{\iint \rho^{\prime} d z d \eta}
$$

There are several other possible methods for calculating the axis of the stream or the downstream coordinate and approximating the external pressure gradient. The geographical center of each section is consistent with the definition of the curvilinear

\footnotetext{
${ }^{12}$ Note that using the center of mass gives virtually identical coordinates.
} 
coordinates (i.e. the edges of the flow are at $\eta \pm L$ ). The center of mass or the center of mass flux is more appropriate from a physical point of view, however. The center of density anomaly was chosen here for dynamical considerations because forces acting on a solid body will act on the center of mass. The density anomaly was chosen because it is dynamically coupled to the center of mass and mass flux but is less sensitive to errors from defining arbitrary limits of the outflow. For instance the center of mass depends on the boundary and may include fluid that is not dynamically active. Also, as we saw with equation 2.14 , the center of density anomaly is the natural choice to approximate the external pressure gradient term.

The total stress on the left hand side of equation 2.13 is thus estimated based on the center of density anomaly of the flow. Variations in the average position or center of each section led to slightly different downstream differences between sections. Therefore, the other possible downstream distances described above led to different total stress estimates. For instance, sections A and B are only $10 \mathrm{~km}$ apart so changing the estimate of downstream distance by $2 \mathrm{~km}$ changes the stress estimate by $20 \%$. In general, the uncertainty in the stress estimate was the largest where the stress estimates themselves were the largest (i.e. between sections $A$ and $B$ ). The magnitude of the total stress could range by more than $1 \mathrm{~Pa}$ near sections $\mathrm{A}$ and $\mathrm{B}$, but the tendency for a decrease of the total stress downstream is a robust feature, i.e. is independent of the method used to calculate the external pressure gradient term or the downstream distance.

\section{Is this the total stress or just bottom stress?}

The stress estimated as a residual of equation 2.13 is considered to be the total stress acting on the flow. The implications of this stress are discussed in appendix B. Basically, the stress includes all the exchanges of momentum that we cannot explicitly calculate, such as small scale pressure fluctuations. 


\section{Discussion and results}

Using the hydrographic data to estimate the pressure forces and the XCP data to estimate the inertial acceleration, a detailed force balance can be examined and the total stress on the outflow diagnosed using equation 2.13. The downstream derivative is calculated using the center of density anomaly as the axis of the stream (see section 2.6.3 for details). For clarity, let us first consider the layered form of the along-stream momentum equation by assuming that $\mathrm{u}$ and $\rho^{\prime}$ are constant within the outflow of width, $\mathrm{W}$ and that the upper layer is motionless. ${ }^{13}$

Equation 2.13 then becomes,

$$
\partial_{\zeta}\left(\rho_{o} \bar{u}^{2} \bar{h} \mathrm{~W}\right)+\mathrm{W} \partial_{\zeta}\left(\frac{1}{2} g \rho^{\prime} \bar{h}^{2}\right)-g \rho^{\prime} \bar{h} \mathrm{~W} \partial_{\zeta} D=-\mathrm{W}\left(\overline{\tau_{B}}+\overline{\tau_{I}}\right) .
$$

The first term on the right hand side involves changes in the total kinetic energy, $\propto u^{2}$. The second term is an internal potential energy term of a fluid column of height, $\bar{h}$. The internal pressure gradient term is often associated with hydraulic models. On a flat bottom, as the height of the interface decreases downstream the internal pressure is lowered and the fluid accelerates towards the lower pressure. Continuity of mass would also require the flow to accelerate downstream. If the density decreases downstream, the flow is also accelerated down the pressure gradient. The third term on the right hand side we call the external potential energy term because it involves displacement of the fluid vertically. As the depth, D, of the particle increases, the fluid column loses gravitational potential energy. Without friction, the external pressure gradient term would accelerate the flow as it moves down the slope.

The layered pressure gradient terms in equation 2.15 can also be derived more directly as shown in appendix $\mathrm{C}$ by assuming a homogeneous background density.

\footnotetext{
${ }^{13}$ Note that these are the layered equations used in Chapter 3 and to evaluate $\rho^{\prime}$ at the axis of the flow we have technically assumed that the layer is sufficiently thin and the stratification is sufficiently weak that $\frac{\partial \rho_{a m b}}{\partial z} h \ll \rho^{\prime}$ and $\frac{\partial \rho_{a m b}}{\partial z} \alpha W / 2 \ll \rho^{\prime}$ (Smith (1973)).
} 
Each term in this equation can also be thought of as an energy term. The kinetic energy is $\propto u^{2}$, and the potential energy is $\frac{1}{2} g \rho^{\prime} h^{2}$. The external potential energy is $g \rho^{\prime} \delta D_{*}$, where $\delta D_{*}$ is defined as the changes in depth from a reference state which we define as $D_{*}$ at section A. Figure 2.25(a) shows the total energy terms at each section. The kinetic energy (dotted line) is found to be less than the internal potential energy (dashed line) and the external potential energy (solid line) terms. The external potential energy (solid line) dominates the total energy (the sum of kinetic, internal potential and external potential, heavy solid line). As seen in equation 2.15, the downstream change in the total energy, $\mathrm{E}$, is equal to the total stress integrated across each section, $\int\left(\tau_{I}+\tau_{b}\right) d \zeta$. Therefore, the slope of the total energy curve in figure $2.25(\mathrm{a})$ is a measure of this integrated stress. ${ }^{14}$

The actual total stress can be seen by directly evaluating each term in equation 2.13 and is shown in figure 2.25(b). The estimated total stress is in excess of 5 $\mathrm{Pa}$ before section $\mathrm{C}$ and decreases to less than $2 \mathrm{~Pa}$ downstream of section $\mathrm{C}$. The total stress estimate (heavy solid line) is dominated by the external pressure gradient (solid line) except between sections $\mathrm{A}$ and $\mathrm{B}$ and $\mathrm{E}$ and $\mathrm{F}$.

Between section A and B the kinetic energy of the outflow decreases (dotted line). This is consistent with the change of the maximum velocity decreasing from $1.25 \mathrm{~m} / \mathrm{s}$ at section $\mathrm{A}$ to $0.80 \mathrm{~m} / \mathrm{s}$ at section $\mathrm{B}$. This loss of kinetic energy in the absence of other forces suggests a frictional deceleration of $2.5 \mathrm{~Pa}$, an order of magnitude above surface wind stress forcing. The internal potential energy decreases partially due to a decrease in $\rho^{\prime}$ but largely due to a decrease in the outflow height from $\bar{h} \approx 123 \mathrm{~m}$ at section $\mathrm{A}$ to $\bar{h} \approx 96 \mathrm{~m}$ at section $\mathrm{B}$. Without any friction, this internal pressure gradient would accelerate the flow downstream. Since the flow is not accelerated this force must be balanced by a retarding stress of $3.7 \mathrm{~Pa}$. The implied

\footnotetext{
${ }^{14}$ The range of the total stress estimate is about $\pm 1 \mathrm{~Pa}$ from section $\mathrm{A}$ to section $\mathrm{C}$ and \pm 0.5 $\mathrm{Pa}$ further downstream.
} 


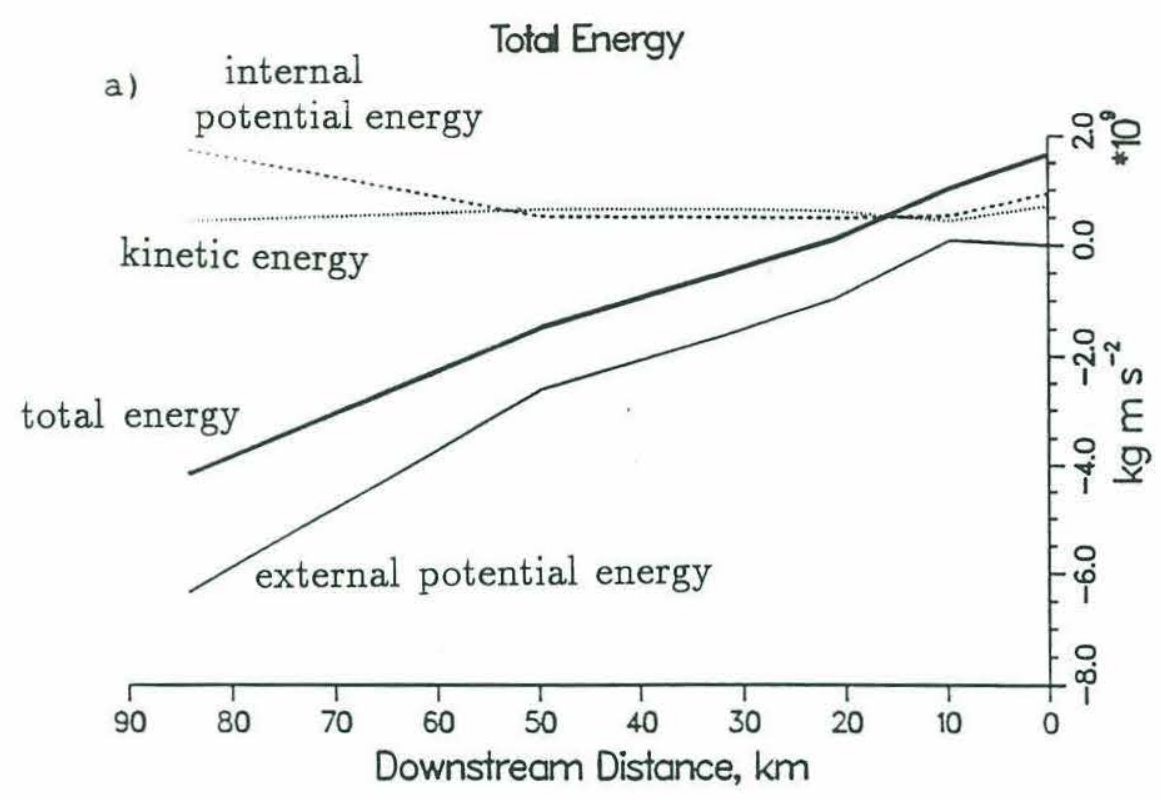

Stress

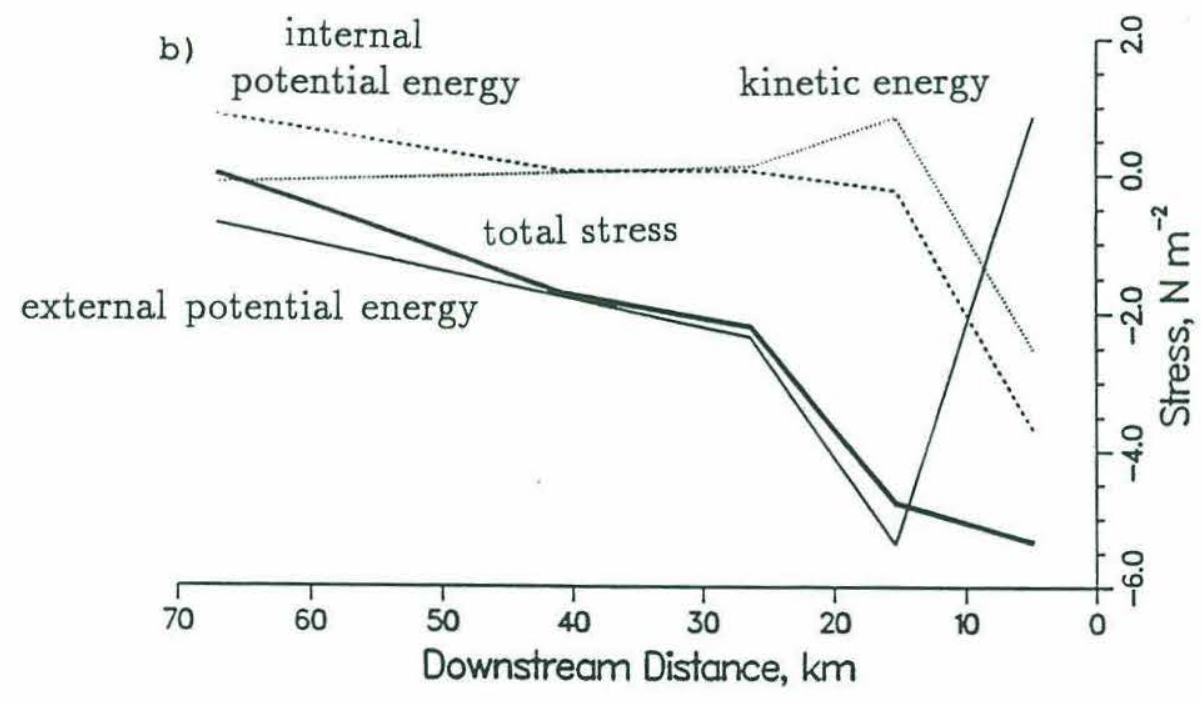

Figure 2.25: Along stream momentum balance. (a) The external potential energy (solid line) decreases as the outflow slides down the continental slope. The internal potential energy (dashed line), changes are mostly reflective of the changes in outflow height. The kinetic energy (dotted line) is an order of magnitude less than the potential energy. The potential energy is not converted into kinetic energy. This loss of total energy (heavy solid line), suggests the presence of large stresses retarding the current. (b) The total stress on the outflow and the contribution of each term in equation 2.13. This calculation suggests a total stress of $5 \mathrm{~Pa}$ retarding the outflow within the first $25 \mathrm{~km}$ of Section A. Further downstream the stress decreases to less than $2 \mathrm{~Pa}$. 
deceleration and decrease in internal potential energy is only partially balanced by an increase in external potential energy. The flow moves slightly uphill as $D_{*}$ decreases from $373 \mathrm{~m}$ at section $\mathrm{A}$ to $367 \mathrm{~m}$ section $\mathrm{B}$ and the external potential energy of the outflow increases (also see figure A.3(a) and figure 2.3(a)). This minuscule uphill excursion of the outflow is not enough to decelerate the flow as observed. From section A to $B$, the net effect of the loss in kinetic and potential energy with the minuscule compensating gain in external potential energy suggests a total stress greater that 5 $\mathrm{Pa}$ must be retarding the flow.

Where the flow first starts its rapid descent down the continental slope the flow accelerates to a maximum speed greater than $1.4 \mathrm{~m} / \mathrm{s}$. The external pressure gradient partially accelerates the flow and increases its total kinetic energy. The descent of the flow is the most rapid near section $\mathrm{C}$ and the along-stream slope is greater than $810^{-3}$. This external pressure gradient should accelerate the flow but doesn't which implies a retarding stress of $4.8 \mathrm{~Pa}$.

The flow continues its descent at a rate of about $5 \cdot 10^{-3}$ until section $\mathrm{E}$ with a retarding total stress of about $2 \mathrm{~Pa}$. Between section $\mathrm{E}$ and $\mathrm{F}$ the flow descends more slowly with a downstream slope decreasing to $1.810^{-3}$. This implies a retarding stress of $1 \mathrm{~Pa}$. This external pressure gradient is balanced by the internal pressure gradient. As we saw from $S_{v w t d}$, the density anomaly does not changes significantly from section $\mathrm{E}$ to $\mathrm{F}$, however, the average height increases from $105 \mathrm{~m}$ to $139 \mathrm{~m}$. This represents an increase in the internal potential energy which is $\approx 1 / 2 g \rho^{\prime} \bar{h}^{2}$ and is consistent with the entrainment observed between sections $\mathrm{E}$ and $\mathrm{F}$ which would raise the center of gravity.

It should be noted however, that the transport decrease between section $\mathrm{A}$ and B that we discussed previously, could be largely responsible for the decrease in kinetic and internal potential energy between these sections. Therefore, the total stress estimated between these sections could be overestimated due to sampling errors and 
time dependence. Similarly, the transport estimated at section F was not consistent with the salt flux at that section, suggesting again either time dependence in the outflow or an overestimate of the transport there. This potential overestimate of transport at section $\mathrm{F}$ would lead to an overestimate of the internal potential energy term. Using different referencing schemes for the absolute velocity field and different estimations of the pressure gradient led to a reduction of the internal pressure gradient term so that the average total stress at section $\mathrm{F}$ was about $0.2 \mathrm{~Pa}$ with a range of \pm 0.3 .

\section{Summary}

The external gravitational term dominates the momentum budget and changes in this term are an order of magnitude greater than changes in the internal pressure gradient or momentum. Therefore, the along-stream momentum budget is a balance between buoyancy and friction. Very large stress greater than $5 \mathrm{~Pa}$ retards the flow before section C. Further downstream stresses less than $2 \mathrm{~Pa}$ allow the flow to slowly cross $\mathrm{f} / \mathrm{h}$ contours. 


\subsubsection{Continuity and Density Equation}

We found in the previous section that very large total stress retards the flow, particularly before section C. We can examine the contribution from the entrainment stress by estimating the entrainment rate, $w_{*}$. The entrainment rate can be estimated by considering the evolution of properties downstream, in particular from the volume flux and salinity flux. Three methods were used to evaluate the entrainment rate. These methods will be described before we discuss the estimated interfacial stress and its implications.

\section{Smith's entrainment coefficient}

The first method to estimate the entrainment rate uses the evolution of the total volume transport downstream as described in equation 2.10. Because the total transport is not monotonically increasing downstream, a least squares fit of transport versus downstream distance was obtained. This is equivalent to fitting the total transport in figure 2.18 to a straight line. We find that the downstream divergence in the total transport is $8.11 \mathrm{~m}^{2} / \mathrm{s}$. To estimate the entrainment rate, $w_{*}$, we divide by the local width (see equation 2.10). This entrainment rate is plotted versus downstream distance in figure 2.26 (solid line).

Note that Smith (1975) parameterizes the continuity equation 2.10 as

$$
\partial_{\zeta} u A=E_{o} u
$$

Dividing the downstream divergence in total transport obtained in the first method by the average $U, 40 \mathrm{~cm} / \mathrm{sec}$, yields an estimate for the average entrainment parameter, $E_{o}$, of $0.02 \mathrm{~km}$. This is smaller than Smith's value of $0.05 \mathrm{~km}$, but within the range calculated by Ambar and Howe (1979b) of $0.02-0.05 \mathrm{~km}$ for the same area. To gain perspective on this entrainment parameter, $E_{o}$, assuming the Gulf Stream has 


\section{Entrainment Rates}

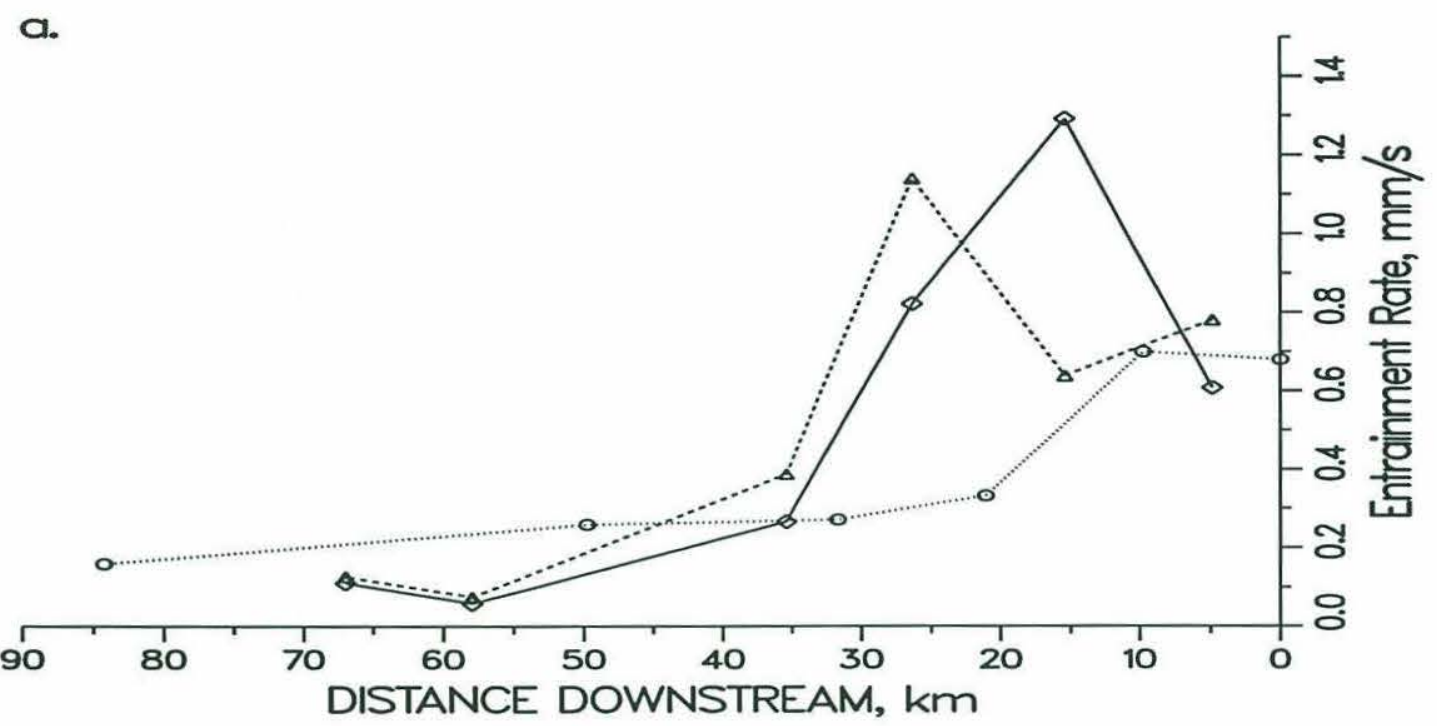

Stress

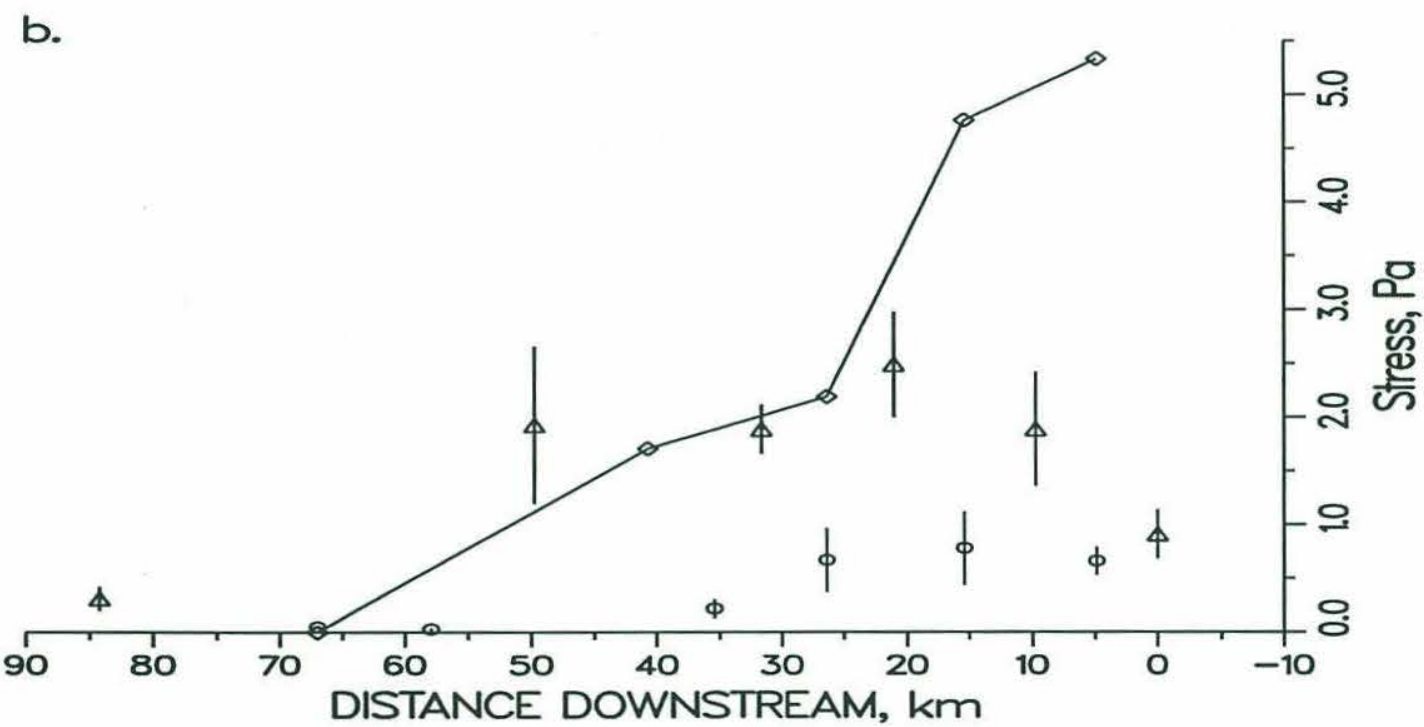

Figure 2.26: a) Entrainment rate, $w_{*}$, estimated three different ways by evaluating the downstream evolution of salinity and volume transport. The solid line is $w_{*}$ from method one which considers $\partial_{\zeta} Q=w_{*}$ W. The dashed line is $w_{*}$ from method two which considers $\bar{u} \partial_{\zeta} \bar{S}=w_{*} S^{\prime}$. The dashed line is $w_{*}$ from method three which considers $\partial_{\zeta} Q_{\text {ent }}=w_{*}$ W. b) Total stress from the momentum budget (solid line), entrainment stress from average of entrainment rate in a) (round symbols), and bottom stress from Johnson et al. (1993) (triangles) obtained from the XCP velocities using the profile method (i.e. fitting to a log layer profile). 
a speed of $1.5 \mathrm{~m} / \mathrm{s}$ this entrainment rate would increase the transport of $30 \mathrm{~Sv}$ in the Florida Straits to $75 \mathrm{~Sv}$ at Cape Hatteras, $1500 \mathrm{~km}$ further downstream. This is consistent with the observed growth of the Gulf Stream for this region but slightly underestimates the increase in transport east of Cape Hatteras which is more rapid (Knauss 1969). ${ }^{15}$

\section{Change in salt flux}

The second method used to estimate the entrainment rate, $w_{*}$, is by the change in salinity downstream. The integrated salinity equation is

$$
\partial_{\zeta} \iint S u d A=-\int_{-l}^{+l} w_{*} S_{\text {ent }} d \eta
$$

Using the continuity equation 2.10 this equation can be rewritten as

$$
\bar{u} \partial_{\zeta} S_{v w t d}=\frac{S^{\prime} w_{*}}{\bar{h}}
$$

where $S_{v w t d}$ is defined in equation 2.2, $\bar{u}$ is the average velocity, and $S^{\prime}$ is the difference between $S_{\text {ent }}$ and $\bar{S}$. $\bar{S}$ for each section is given in table 2.3 and repeated in table 2.4 for convenience. We assume that $S_{\text {ent }}$ is approximately 35.6. The average height, $\bar{h}$, and the average velocity, $\bar{u}$, are given in table 2.4. The resulting entrainment rate is shown in figure 2.26 (dotted line). The entrainment rate reaches a maximum of 1.3 $\mathrm{mm} / \mathrm{s}$ between section $\mathrm{B}$ and $\mathrm{C}$ and decreases rapidly further downstream.

\section{Changes in entrained water, $Q_{\text {ent }}$}

The third method of estimating the entrainment rate also uses the downstream change in transport used in method one. The total transport, however, is modified as we saw

\footnotetext{
${ }^{15}$ This gives you an idea for the intensity of entrainment and is not meant to suggest that the Gulf Stream transport increases by a similar process (or vice versa).
} 


\begin{tabular}{|c|c|c||c|c|c|c||c|c|}
\hline Section & $\bar{S}$ & $\bar{h}$ & $\bar{u}$ & $u_{\text {wtd }}$ & $u_{\text {ke }}$ & $u_{\max }$ & $u_{\text {ent }}$ & $\delta X$ \\
\hline \hline $\mathrm{A}$ & 37.75 & 123 & 0.60 & 0.70 & 0.82 & 1.11 & 0.21 & 0.0 \\
$\mathrm{~B}$ & 37.51 & 96 & 0.59 & 0.59 & 0.68 & 0.78 & 0.23 & 9.8 \\
$\mathrm{C}$ & 37.10 & 87 & 0.34 & 0.48 & 0.63 & 1.11 & 0.08 & 21.1 \\
$\mathrm{D}$ & 36.60 & 101 & 0.34 & 0.38 & 0.62 & 0.92 & 0.09 & 31.7 \\
$\mathrm{E}$ & 36.65 & 105 & 0.32 & 0.38 & 0.63 & 0.71 & 0.13 & 49.8 \\
$\mathrm{~F}+\mathrm{FE}$ & 36.51 & 139 & 0.18 & 0.26 & 0.32 & 0.43 & 0.01 & 84.2 \\
\hline
\end{tabular}

Table 2.4: Section averaged properties of the Mediterranean outflow. $\bar{S}$ is the transport weighted salinity as described in section 2.5 and table 2.3 and repeated here for convenience. The averaged height is $\bar{h}$ in meters. The average velocity is $\bar{u}$. $u_{w t d}$ is the flux weighted average velocity. $u_{K E}$ is the kinetic energy average velocity obtained by dividing the integrated kinetic energy (i.e. in equation 2.13) by the transport. $u_{\max }$ is the flux weighted maximum velocity. The flux averaged entrainment velocity is $u_{\text {ent }}$ which represents the velocity just above the outflow. All velocities are in $\mathrm{m} / \mathrm{s} . \delta X$ is the distance downstream from section $\mathrm{A}$ in $\mathrm{km}$.

in section 2.5 by reconciling the inconsistency between the total transport estimates and the salt flux. We assume that each section contains a constant flux of $0.4 \mathrm{~Sv}$ of pure Mediterranean water, $Q_{\text {med }}$. This gives us the function $Q_{\text {ent }}$ listed in table 2.3 which is monotonically increasing except at section D. A least square fit between $Q_{\text {ent }}$ and distance downstream yields a slope of $7.6 \mathrm{~m}^{2} / \mathrm{s}$, which is very similar to the slope obtained in the first method and gives essentially the same entrainment coefficient, $E_{o}=0.19 \mathrm{~km}$. Because $Q_{\text {ent }}$ is more consistent with the net salt flux at each section, however, we can estimate $w_{*}$ as a function of downstream distance using $\partial_{\zeta} Q_{e n t}=w_{*} \bar{W}$. The entrainment rate is given in figure 2.26 (dashed line) which shows $w_{*}$ reaches a maximum of $1.1 \cdot 10^{-3} \mathrm{~m} / \mathrm{s}$ between sections $\mathrm{C}$ and $\mathrm{D}$ where we found the large transport increase.

\section{Results}

Estimations of the entrainment rate that use the relatively stable salinity structure include both method two, which considers the downstream changes in salinity, and 
method three, which considers the change in transport that conserves salt flux. These entrainment rates show a very similar structure with a maximum $w_{*}$ between sections $\mathrm{B}$ and $\mathrm{D}$ and a rapid decrease to values an order of magnitude smaller only $30 \mathrm{~km}$ further downstream. Method one, however, does reach a slight maximum of $0.7 \mathrm{~mm} / \mathrm{s}$ which is almost half of the other maximum entrainment rates. This is primarily due to the least squares approach which assures that the transport increases uniformly downstream, which we have seem is clearly not a good assumption.

These entrainment rates are quite high relative to entrainment observed elsewhere in the worlds oceans. An entrainment of $1 \mathrm{~mm} / \mathrm{s}$ for instance translates into approximately $30 \mathrm{~km} /$ year. Typical Ekman pumping values are between 25-50 m/year and subduction rates are slightly larger at approximately 50-100 m/year over the North Atlantic (Marshall et al., 1993). The entrainment into the overflow is then on the order of 1000 times larger than typical entrainment rates driving the thermocline circulation. We see that the entrainment into the outflow is very localized spatially, however. Area integrated rates throughout the whole Gulf of Cadiz would reach a maximum of $2 \mathrm{~Sv}$ whereas the subduction rate averaged over the area of the North Atlantic is between $30-50 \mathrm{~Sv}$ (R. Williams, personal communication).

Entrainment rates, $w_{*}$, can be converted to interfacial stress estimates as we saw in section B. The interfacial stress, $\tau_{I}$, is $\rho_{o}\left(u-u_{a b o v e}\right) w_{*}$ for a layered model. The observed outflow is not layered with uniform velocity of density, however. The difficulty in obtaining an interfacial stress estimate lies in the uncertainty of the appropriate velocity of the layer to use. Here, four different estimates of the outflow velocity are used:

- $\bar{u}$, the average velocity, which is determined by the total transport divided by the area, 
- $u_{w t d}$, the flux weighted average velocity, in which the average velocity at each station is weighted by the flux at that station

- $u_{K E}$, the normalized 'kinetic energy' velocity determined by dividing the total section integrated kinetic energy (i.e. equation 2.13) by the total section integrated transport, and

- $u_{\max }$, the flux weighted maximum velocity, in which the maximum velocity at each station is weighted by the flux at that station

These average velocity estimates are shown in table 2.4 . For each $w_{e}$ value we have four estimates of the interfacial stress. To obtain an average stress estimate we use the $w_{e}$ estimates from method two and three, the evolution of salinity and the downstream divergence of the entrained water, $Q_{\text {ent }}$. We omit the entrainment rate obtained from the least square fit of the total transport with downstream distance (method one), because we know that the transport increase is not uniform downstream.

The average interfacial stress is shown in figure 2.26(b) (open circles). The error bars represent the standard deviation of the eight stress estimates at each downstream location. The total stress obtained in section 2.6.3 and shown in figure 2.25(b) is reproduced in figure 2.26(b) (solid line and diamond symbols). The interfacial stress reaches a maximum of $0.8 \mathrm{~Pa}$ between section $\mathrm{B}$ and $\mathrm{C}$ with stresses greater than 0.7 $\mathrm{Pa}$ from section $\mathrm{A}$ to section $\mathrm{D}$. The interfacial stress decreases rapidly downstream of section $\mathrm{D}$ to less $0.05 \mathrm{~Pa}$. The interfacial stress contributes largest to the total stress where the total stress is largest.

The difference between the total stress and the interfacial stress should equal the bottom stress. For comparison, the bottom stress estimates of Johnson et al. (1993) are plotted in figure 2.26(b) (triangular symbols with standard deviation error bars). The bottom stress estimates were obtained by fitting the XCP profiles in this experiment to a log layer velocity profile. The bottom stress reaches a maximum of 
$2.5 \mathrm{~Pa}$ at section $\mathrm{C}$ with values in excess of $1.9 \mathrm{~Pa}$ from section $\mathrm{B}$ to $\mathrm{E}$. The sum of bottom stress and interfacial stress reaches only $2-3.5 \mathrm{~Pa}$ between sections $\mathrm{A}$ and $\mathrm{B}$ which is much less than the total stress estimate of $5 \mathrm{~Pa}$. Further downstream, however, the comparison is much better and lies within the expected error. The largest uncertainty in the total stress derived from the momentum budget is as large as $1 \mathrm{~Pa}$ before section $\mathrm{C}$ and decreasing to $\pm 0.5 \mathrm{~Pa}$ further downstream. In general, the bottom stress dominates the total stress and the largest interfacial stress occurs where the mixing is intense.

\subsection{Conclusions}

The overall structure of the outflow is similar to that described in the historical literature such as Heezen and Johnson (1969) or Madelain (1970), confirming the fairly steady nature of the outflow over decades. The outflow lies entirely on the continental slope during its initial descent into the Gulf of Cadiz where it mixes with fresh North Atlantic water, reducing its salinity anomaly and increasing its transport. During the first $100 \mathrm{~km}$ of descent the overflow transport doubles from $0.85 \mathrm{~Sv}$ to $1.9 \mathrm{~Sv}$ and the overflow broadens from $10 \mathrm{~km}$ to $90 \mathrm{~km}$. Cross stream differences in the overflow T/S properties increase as the current spreads. Slightly differentiated water types exit the Strait with the saltiest, coldest outflowing water to the south and slightly fresher and warmer outflow to the north (Figure 2.13(a)). Since the northern, near shelf current (core) is higher in the water column, it mixes with warmer North Atlantic water than does the deeper offshore current. What begins as less than a $0.5^{\circ} \mathrm{C}$ cross stream variation in water types in the Strait becomes more than a $2^{\circ} \mathrm{C}$ variation as the current spreads within the first $100 \mathrm{~km}$ (Figure 2.13(f)). The outflow eventually settles along two preferred isopycnals: 27.5 and 27.8 (Zenk 1975b). 
The maximum core salinity is 38.4 pss at the Strait and decreases by 0.6 pss in the $40 \mathrm{~km}$ between section $\mathrm{A}$ and section D. Combining hydrographic data with the current measurements suggests a much more intense mixing process than the decay in the core salinity implies. The velocity-weighted salinity of the outflow begins with a salinity of 37.7 pss near the Strait and decreases over 1 pss within the first $40 \mathrm{~km}$ (Figure 2.17). The difference between the core salinity values and the velocityweighted salinities is due mostly to the stratified nature of the outflow. Core salinity values, which are located within $10 \mathrm{~m}$ of the bottom, do not accurately reflect the average properties of the $100 \mathrm{~m}$ thick outflow because the highest speeds are above the maximum salinity. The overflow becomes neutrally buoyant near Cape St. Vincent with a core salinity of $36.6 \mathrm{pss}$, over $300 \mathrm{~km}$ from the Strait. Since the velocityweighted salinity has already attained a value close to this only $40 \mathrm{~km}$ from the Strait, most of the mixing has taken place within these first $40 \mathrm{~km}$.

The criterion for Kelvin Helmholtz instability is that the gradient Richardson number is less than $\frac{1}{4}$. Low gradient Richardson numbers are found in the well mixed outflowing layer where the stratification is very weak and also near the interface between outflowing water and the return circulation above (Figure 2.15). At the interface, the stratification is extremely strong but the velocity shear is so large it could overcome the stability of the buoyancy forces. These low gradient Richardson numbers suggest the current is unstable to Kelvin Helmholtz instability and mixing through this mechanism may occur. Bulk Richardson numbers or equivalently bulk Froude numbers support this theory (Figure 2.15 and 2.22). Supercritical Froude numbers, Fr $>1$, were found near the Strait coincident with the vigorous mixing observed through the salinity and transport calculations. Further from the Strait, lower bulk Froude numbers were observed in regions where less entrainment is taking place (Figure 2.23). Coincidence of low gradient Richardson numbers and large bulk Froude numbers in regions of observed mixing support the conclusion that mixing is driven by Kelvin Helmholtz instability. 
Near the Strait, advection, dissipation and the Coriolis force all make important contributions to the dynamics of the outflow. Further downstream, the flow becomes a slightly damped geostrophic current. After the Mediterranean water exits the Strait it takes a right hand turn into the Gulf of Cadiz. This deflection of the current is a nearly inertial turn in the current, where the advection terms are the same order of magnitude as the pressure gradient. Examination of the downstream momentum flux divergence suggests an average stress of $2 \mathrm{~Pa}$ retarding the current within the first $100 \mathrm{~km}$ of descent. Near the Strait, the stress may be as high as 5 $\mathrm{Pa}$ while further downstream the stress decreases to less than 0.5 Pa (Figure 2.25). Most of the potential energy released from the current is due to the descent of the current down the continental slope. This pressure gradient is balanced by frictional dissipation instead of increasing the kinetic energy of the mean flow. The interfacial stress on the flow reaches a maximum of $0.8 \mathrm{~Pa}$ near section $\mathrm{C}$ where the flow has accelerated to maximum speeds above $1.4 \mathrm{~m} / \mathrm{s}$. The acceleration of the flow increases the bulk froude number to supercritical values and the flow mixes intensely. Thus we find the very localized mixing along the total path of outflow in the Gulf of Cadiz. 


\section{Chapter 3}

\section{A Simulation of the}

\section{Mediterranean Outflow}

In chapter two, we examined data that showed the Mediterranean water flowing out through the Strait of Gibraltar, as a dense plume cascading down the bottom slope and mixing with the overlying water. The Mediterranean plume slowly loses its high salinity as it mixes with the fresh North Atlantic water and flows northwest toward Cape St. Vincent where it becomes neutrally buoyant. We are interested in understanding this mixing process and the dynamics which control the plume. In this chapter we use a simple numerical model to investigate the sensitivity of the outflow characteristics to variations in the boundary conditions at the strait. This model was developed in collaboration with J. Price, and is described briefly in Price and Baringer (1993, hereafter PB93). Here we compare the simple plume model with data taken during the Gulf of Cadiz Expedition (described in chapter 2, hereafter GofCExp), examine the momentum and energy balances, and assess several parameterizations of broadening. 

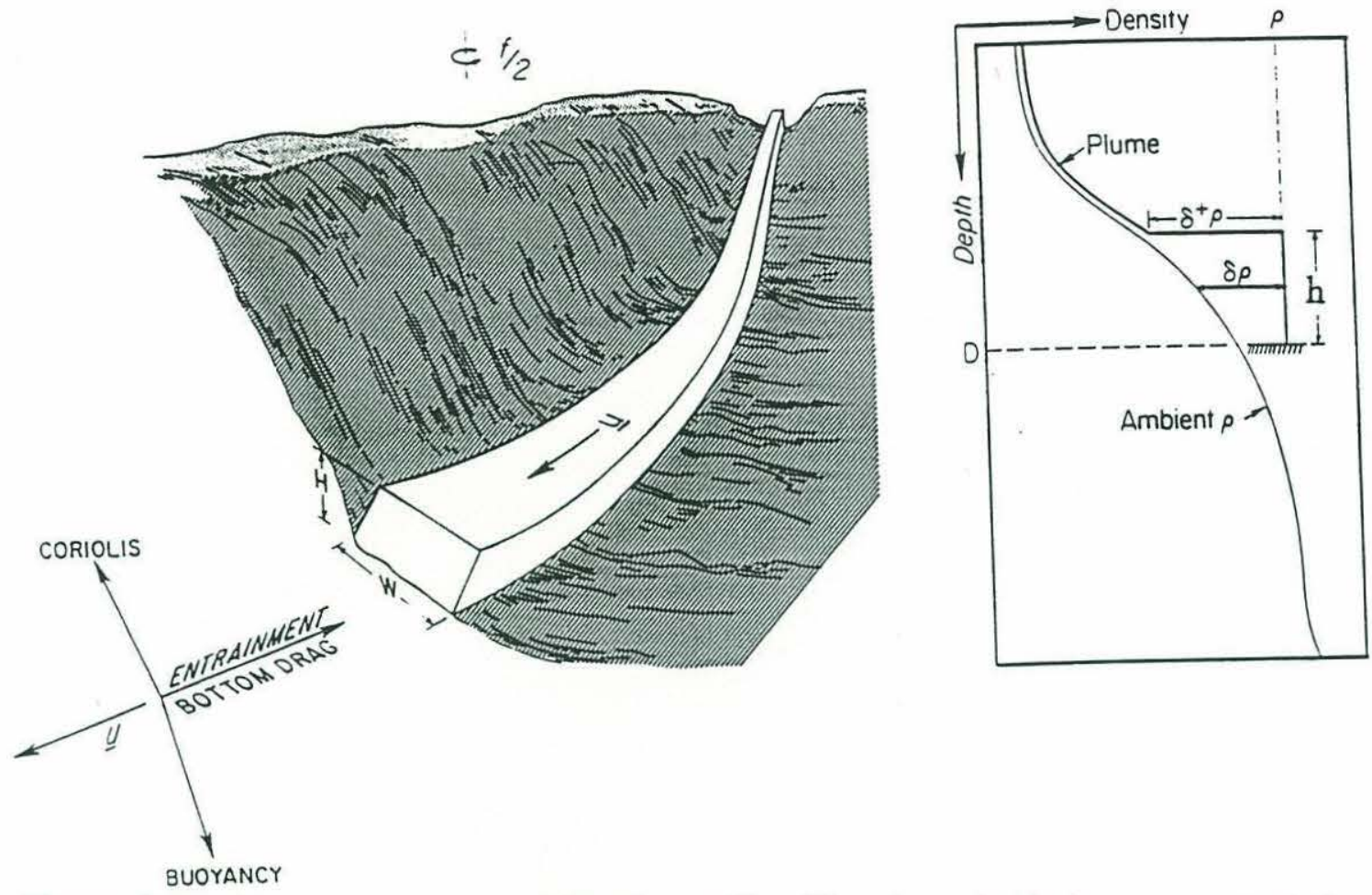

Figure 3.1: Model geometry and density profile. The plume is idealized as a single layer of height $\mathrm{H}$ and width $\mathrm{W}$. It is allowed to flow freely down the continental slope beginning at the western end of the Strait of Gibraltar (reproduced from Price and Baringer, 1993).

\subsection{Model Formulation}

The model consists of a homogeneous bottom layer representing the plume, surrounded by a continuously stratified, inactive upper ocean (figure 3.1). In this sense, it is a reduced gravity model with the active layer on the bottom. The model includes rotation, mixing into the plume, quadratic bottom friction and realistic bottom topography. The entrainment stress is of the form given by Ellison and Turner (1959) and the entrainment rate is parameterized using a Froude number formulation (Turner 1986). The model is integrated across-stream and vertically so that for each downstream position we specify one value of $u, v, h, W, T$ and $S$. Therefore, we are predicting the evolution of the bulk properties of the flow as the fluid moves down the continental slope and mixes with the ambient stratification and not the detailed 
structure. The model equations are integrated along the stream axis to yield velocity, temperature and salinity for each model realization.

A schematic of the model is shown in figure 3.1. The model is steady and allowed to move freely down the continental slope with bottom depth given by $D(x, y)$. The flow moves through passive ambient stratification, $\rho_{a m b}$ which depends on $z$ but is independent of horizontal position. Given these assumptions, the momentum equations are given by

$$
\begin{aligned}
\vec{u} \cdot \nabla u & =\frac{g \delta \rho}{\rho_{o}} \frac{\partial D}{\partial x}+f v-\frac{\tau_{b}^{x}}{\rho h}-\frac{\tau_{e}^{x}}{\rho h} \\
\vec{u} \cdot \nabla v & =\frac{g \delta \rho}{\rho_{o}} \frac{\partial D}{\partial y}-f u-\frac{\tau_{b}^{y}}{\rho h}-\frac{\tau_{e}^{y}}{\rho h}
\end{aligned}
$$

For simplicity the Coriolis parameter, $f$, is a constant (an f-plane).

The model geometry is similar to the models of Smith (1975) and later Killworth (1977). The most important difference is that the friction and entrainment terms are determined from the flow field and therefore depend critically on the specific properties of the flow. Smith and Killworth's models are essentially diagnostic because the friction and entrainment terms are fitted to the data, while this model predicts entrainment and friction from simple bulk formulae.

\section{Buoyancy}

The first term on the right hand side of equation 3.1 and 3.2 represents the buoyancy forcing. See Smith (1973) and section 2.6.3 for the full derivation of this pressure gradient term. We have assumed that,

$$
g \delta \rho h \nabla D \gg \nabla\left(\frac{1}{2} g h^{2} \delta \rho\right)
$$

In appendix $\mathrm{C}$ we show a simplified derivation of the pressure gradient using a uniform outflow layer with constant ambient density that emphasizes that the gradient of the 
density anomally must be included in the right hand side of equation 3.3. We also examine the validity of this approximation (appendix C). For now, we note that this is valid when the changes in the bottom slope are much greater than the changes in the interface depth. With a flat bottom, this 'internal pressure gradient' term would drive the flow as in hydraulic models. Thus we have restricted ourselves to relatively steep slopes and therefore cannot initialize our model in the upstream basin, or at the sill where the slope would vanish. The omission of this term filters gravity waves from the model and thus the model cannot be hydraulically controlled and the upstream conditions cannot be influenced by the downstream conditions. This approximation, although unsatisfying, is necessary for the simple lagrangian solution technique employed here where the upstream conditions are held fixed and the flow is followed downstream. Including this term makes the equations elliptic and requires a two dimensional inversion and thus a higher dimensional model (see appendix $\mathrm{C}$ for a more thorough discussion).

Since the parcel of fluid moves through ambient stratification, the method of calculating the density anomaly must be clarified. In the entrainment term the in situ density difference is calculated at the top of the outflow, where the layer is in contact with the ambient stratification. The buoyancy anomaly however, is calculated at the mid-depth of the layer (see figure 3.1) ${ }^{12}$.

\section{Bottom stress}

The bottom stress is modeled by a quadratic stress law, $\vec{\tau}_{b}=\rho c_{d}|\vec{u}| \vec{u}$. We choose $c_{d}=0.003$, the canonical value (Sternberg, 1968). Figure 3.2 shows how the bottom stress varies with speed and the bulk Froude number, $F r_{b}$. The speed is varied while

\footnotetext{
${ }^{1}$ An integrated vertical density anomaly represents the 'true' buoyancy forcing better (see section 2.6.3) but for constant stratification they are identical (see appendix C).

${ }^{2}$ In using the mid-column density difference we have assumed that the layer is sufficiently thin and the stratification is sufficiently weak that $\frac{\partial \rho_{a m b}}{\partial z} h \ll \rho^{\prime}$ and $\frac{\partial \rho_{a m b}}{\partial z} \alpha W / 2 \ll \rho^{\prime}$ (Smith (1973)).
} 
the other parameters are held fixed (see figure caption). The bottom stress increases gradually as the square of the speed.

There is however, some uncertainty in the precise value of $c_{d}$. Historical estimates in shallow seas and bays range from $1.73 \cdot 10^{-3}$ in the Irish Sea (Heathershaw, 1976) to $5.4 \cdot 10^{-2}$ in the Chesapeake Bay (Ludwick, 1975). Using data from the Gibraltar Expedition discussed in chapter two, Johnson et al. (1993) estimated the bottom stress using XCP velocities fit to a logarithmic velocity profile in the constant stress layer, and an expendable dissipation profile that measures turbulent intensity directly. They found a range in the value of $c_{d}$ with an average of $2.5 \pm 0.7 \cdot 10^{-3}$. Although there is considerable variability in the estimates of $c_{d}$, we assume that this range is caused by instrument error and temporal variability and that $c_{d}$ is 'known'; we choose not to 'fit' the data to the 'best' value but instead chose the most often quoted value of 0.003 .

\section{Entrainment}

The velocity of the layer is also reduced by entrainment stress, $\overrightarrow{\tau_{e}}=\rho w_{e} \vec{u}$, where $w_{e}$ is the entrainment velocity into the layer. In a reduced gravity model, the momentum of an entraining layer is conserved, but the velocity of the layer is reduced as the layer mixes with non-moving water. Thus, entrainment acts to reduce the average velocity. The entrainment rate is parameterized as

$$
w_{e}= \begin{cases}\frac{0.8-0.1 R_{b}}{1+5 R_{b}}|\vec{u}| & \text { if } R_{b} \leq 0.8 \\ 0.0 & \text { otherwise }\end{cases}
$$

where $R_{b}=\frac{g \delta \rho^{+}}{\rho_{o}} h /|\vec{u}|^{2}$, the bulk Richardson number of the layer, which is evaluated following the flow. The density anomaly, $\delta \rho^{+}$, is defined as the density difference at the top of the plume. This entrainment parameterization is based on laboratory experiments of Ellison and Turner (1959) and Turner (1986). Equation 3.4 can be 


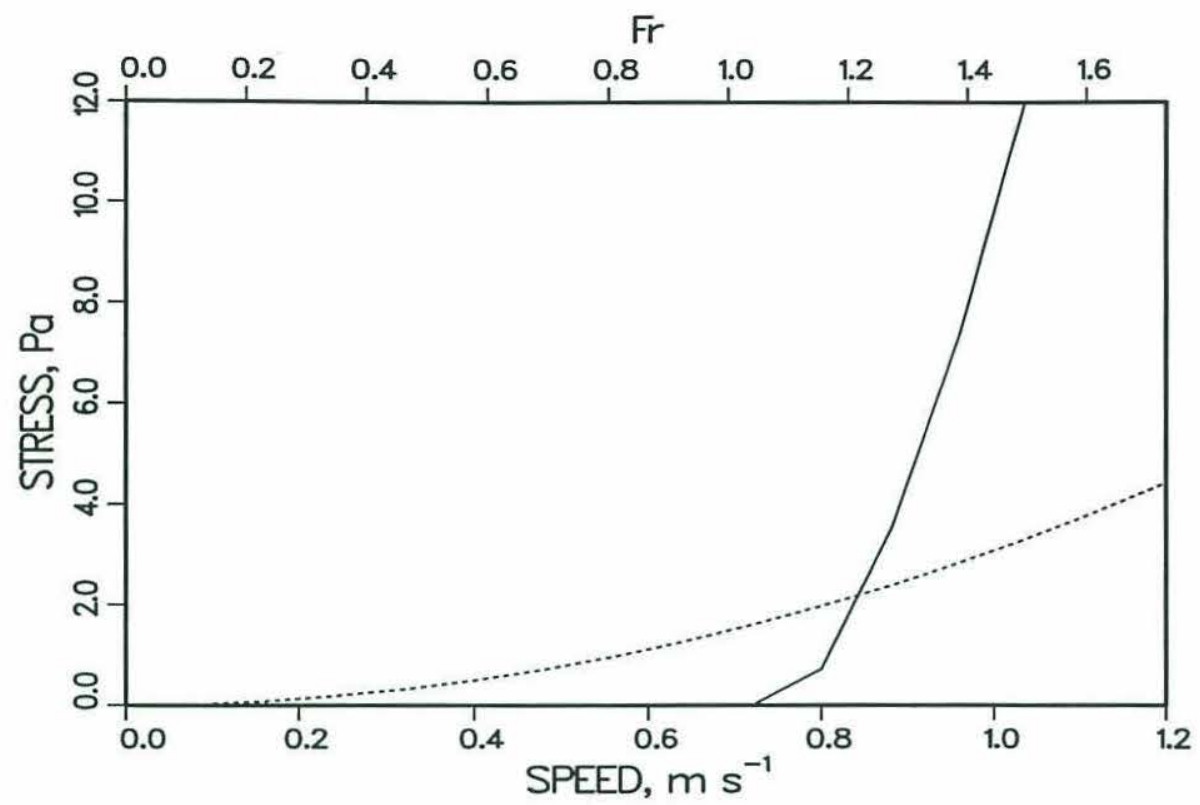

Figure 3.2: Entrainment and Bottom Stress versus Froude number. Solid line is the entrainment stress given by the entrainment rate in equation 3.4. The dashed line is the bottom stress given by the quadratic drag law. Stresses are shown as a function of velocity and $F r_{b}$. The speed is varied while the other parameters are held fixed $\left(F r_{b}=|\vec{u}| / \sqrt{\frac{g \delta \rho^{+}}{\rho_{o}} h}\right.$, $\left.h=100 \mathrm{~m}, \delta \rho^{+}=0.5 \mathrm{~kg} / \mathrm{m}^{2}, g=9.8 \mathrm{~m} / \mathrm{s}^{2}, \rho_{\circ}=1023 \mathrm{~kg} / \mathrm{m}^{3}, c_{d}=0.003\right)$.

written equivalently for a bulk Froude number, $F r_{b}=R_{b}^{-\frac{1}{2}}$. This formulation couples the entrainment with the flow properties allowing prediction of the entrainment rate without tuning.

Figure 3.2 also shows how the entrainment varies with speed and $F r_{b}$. The speed is varied while the other parameters are held fixed (see figure caption). The bottom stress increases gradually as the square of the speed. The entrainment stress also strengthens with increasing speed but grows abruptly as the $F r_{b}$ exceeds 1 . This corresponds to $R_{b}$ becoming smaller, especially falling below 0.65 . Note that Price et al. (1986) use a bulk Richardson number parameterization that forces the surface mixed layer to mix until the $R_{b}$ is greater than this critical value of 0.65 . The key consequences of this entrainment parameterization are that 
- the entrainment depends critically on the flow parameters,

- the entrainment can be intermittent, and

- no instantaneous adjustment is required (the flow can remain unstable over a period of time).

\section{Thermodynamics}

The model treats temperature and salinity separately and uses a fully nonlinear, pressure dependent equation of state,

$$
\rho=\rho(T, S, p) \text {. }
$$

This includes compressibility effects that are not significant in the Gibraltar region, but are critical for modeling northern overflows that descend thousands of meters. This so called 'thermobaric effect' has been shown by Killworth (1977) to be essential in the descent of deep water in the Weddell Sea. The ambient stratification is separated into temperature and salinity profiles. The temperature and salinity are modified by the entrainment of the ambient stratification through the top of the layer. Diffusion is neglected relative to the much stronger entrainment process. The temperature and salinity equations are

$$
\begin{aligned}
& \vec{u} \cdot \nabla T=-w_{e} \delta^{+} T / h \\
& \vec{u} \cdot \nabla S=-w_{e} \delta^{+} S / h
\end{aligned}
$$

where $\delta^{+} S, \delta^{+} T$ are defined as the difference in salinity and temperature between the layer and the ambient stratification at the top of the layer. 


\section{Continuity equation}

To close the system, we need equations for the height, $h$ and the width, $W$. We use the continuity equation to predict $h$, which can be written in flux form as,

$$
\nabla \cdot(\vec{u} h W)=w_{e} W
$$

We see that following the fluid downstream, the volume flux can only be changed by a flux through the surface of the layer.

\section{Width Equation}

We are left with the question of how to specify the width of this integrated layer. In this chapter we will examine two alternative explanations for the spreading of the outflow. We will take up later the inviscid end of the spectrum where we assume that the flow is geostrophic and has uniform potential vorticity that is conserved (chapter 3.5). In PB93 it was assumed that bottom drag governs the spreading of the flow. We term this spreading mechanism the 'Ekman spreading'. We can express the balance between the Coriolis and frictional forces by considering the $\mathrm{x}$-axis aligned along isobaths and the $\mathrm{y}$-axis downslope, parallel to the pressure gradient as shown in figure 3.3. The $\mathrm{x}$-momentum equation is then

$$
f v \approx \frac{c_{d}|\vec{u}| u}{h} .
$$

and the $y$-momentum equation contains the buoyancy term. Note that the ratio of buoyancy force (or the Coriolis force) to friction can be represented as an Ekman number. Then, assuming the configuration in figure 3.3,

$$
\frac{v}{u} \approx \frac{c_{d}|\vec{u}|}{f h} \approx \tan (\beta),
$$

where $\beta$ is the angle the parcel makes relative to a purely geostrophic flow. 

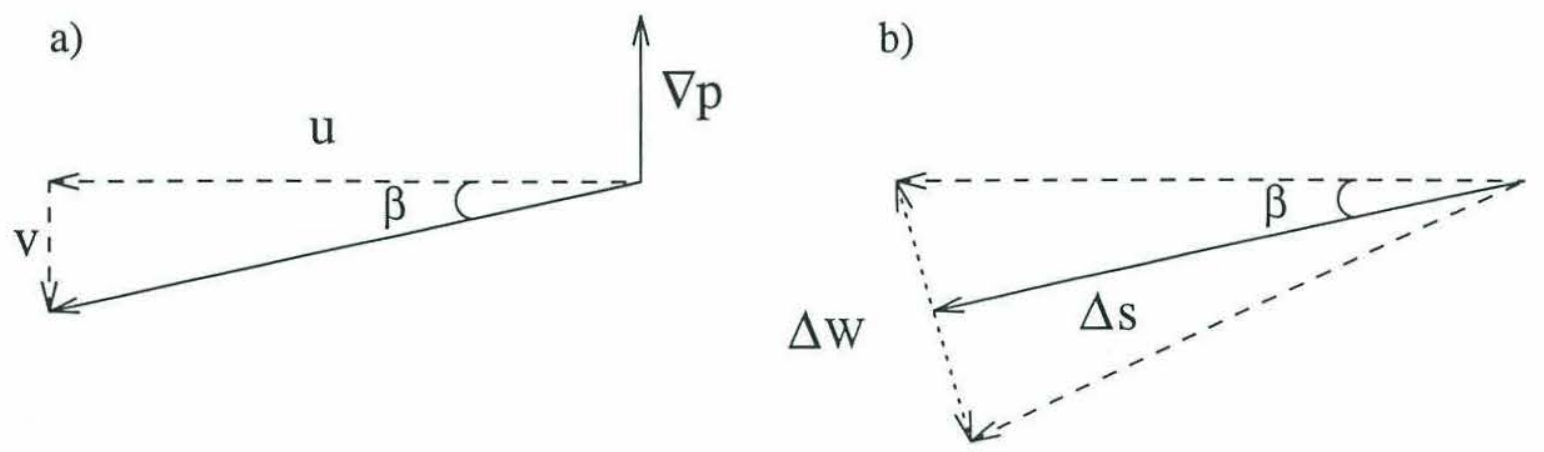

Figure 3.3: Schematic of spreading of layer as a result of friction. a) The solid line is the velocity resulting from friction, buoyancy and the Coriolis force. For convenience, we assume that the pressure gradient is aligned with the y axis (v velocity). The angle the velocity vector makes relative to a pure geostrophic balance is defined as $\beta$. b) the upper edge of the layer is assumed to follow isobaths, the average position of the outflow moves down-slope at an angle $\beta$, and the lower edge of the flow descends at twice $\beta$.

From the observations discussed in chapter 2 the shallow edge of the outflow crosses isobaths very little, while the deeper, offshore edge of the flow descends more rapidly. Here, we have assumed that the center of the flow crosses isobaths at a rate given by the Ekman number, $E^{1 / 2}=\frac{c_{d}|\vec{u}|}{f h}$, and the upper edge of the flow follows isobaths. The width then can be determined from the geometric considerations as shown in figure 3.3. We have

$$
\frac{\frac{1}{2} \Delta W}{\Delta s}=\tan (\beta)
$$

where $\Delta s$ is the distance the parcel has moved. Multiplying through by $\frac{\Delta s}{\Delta t}$ and taking the limit as $\Delta t \rightarrow 0$, we have

$$
\begin{aligned}
\frac{d W}{d t} & =2|\vec{u}| \frac{c_{d}|\vec{u}|}{f h} \\
& =2|\vec{u}| E^{1 / 2}
\end{aligned}
$$

This specification of the width depends critically on frictional spreading and the value of $c_{d}$. Some inviscid geostrophic adjustment process could also govern the spreading of the overflow. We will examine this possibility in section 3.5. Topography could also constrain the flow by inhibiting spreading. In the Mediterranean we saw 
that the outflow between sections A through $\mathrm{C}$ was confined in a channel. Therefore, for the initial $20 \mathrm{~km}$ downstream, we set $2 E^{1 / 2}=0.1$ to restrict the broadening of the flow as observed in the data. Surprisingly, we find that the final temperature, salinity and transport of the mixed Mediterranean water is fairly insensitive to whether we prescribe geostrophic or frictional spreading and the details of the channel (see section 3.5).

\subsubsection{Implementation}

The model equations to be solved are

$$
\begin{aligned}
\vec{u} \cdot \nabla u & =\frac{g \delta \rho}{\rho_{o}} \frac{\partial D}{\partial x}+f v-\frac{c_{d}|\vec{u}| u}{h}-\frac{w_{e} u}{h} \\
\vec{u} \cdot \nabla v & =\frac{g \delta \rho}{\rho_{o}} \frac{\partial D}{\partial y}-f u-\frac{c_{d}|\vec{u}| v}{h}-\frac{w_{e} v}{h} \\
\nabla \cdot(\vec{u} h W) & =w_{e} W \\
\rho & =\rho(T, S, p) \\
\vec{u} \cdot \nabla T & =-w_{e} \delta^{+} T / h \\
\vec{u} \cdot \nabla S & =-w_{e} \delta^{+} S / h \\
w_{e} & = \begin{cases}\frac{0.8-0.1 R_{b}}{1+5 R_{b}}|\vec{u}| & \text { if } R_{b} \leq 0.8 \\
0.0 & \text { otherwise }\end{cases} \\
R_{b} & =\frac{g \delta \rho^{+} h}{|\vec{u}|^{2}} \quad 2 \quad \text { if } \quad\left|\vec{x}-E^{1 / 2}\right| \geq 20 \mathrm{~km} \\
\frac{d W}{d t} & =2 \begin{array}{ll}
\frac{c_{d}|\vec{u}|}{f h} & \text { otherwise } \\
0.05 &
\end{array}
\end{aligned}
$$

Note that for simplicity and clarity we have rewritten the entire set here. Each equation was discussed individually in section 3.1. 
To integrate this set of equations, we use the fact that in a steady state particle trajectories are the same as streamlines. Then $\vec{u} \cdot \nabla$ can be written as $\frac{d}{d t}$ and we can integrate forward in time following a particle. The position of the flow can be determined by integrating the model velocity in time,

$$
\vec{x}=\overrightarrow{x_{0}}+\int_{0}^{t} \vec{u} d t
$$

All prognostic equations are stepped forward using an Euler forward scheme, where all the terms on the right hand side are calculated at the previous time step. Therefore, the order of integration is unimportant. The only boundary condition necessary for this hyperbolic problem are the initial conditions at $t=0$. The initial conditions for the Mediterranean outflow are listed in table 3.1. Initial model variables are denoted with a subscript. For instance, $u(t=0)=u_{o}$.

The calculation of all the terms on the right hand side of our model equation set are quite straightforward. The pressure gradient term is the only term that requires comment. The actual Gulf of Cadiz bottom topography is very rough with many canyons, and cannot be well represented by a flat plane. Accordingly, we use a digitized five minute $(8 \mathrm{~km})$ resolution topography of the Gulf of Cadiz, somewhat smoothed, as shown in figure 3.6. The slope of the topography is taken from this digitized data set by calculating the average slope across the entire width of the outflow (i.e. at each downstream location the slope is the average slope over that cross section).

\subsubsection{Numerical stability}

Consider a simplified form of the above momentum equations.

$$
\frac{d \vec{u}}{d t}+\vec{f} \times \vec{u}=-k \vec{u}
$$


where the friction coefficient, $k$, is equivalent to $c_{d}|\vec{u}| / h$ in the full equations above. Let the velocity grow with an amplification factor $\lambda$, so that

$$
\vec{u}^{n+1}=\lambda \vec{u}^{n}
$$

where the superscript, $\mathrm{n}$ and $\mathrm{n}+1$ represent the time steps. Substituting equation 3.22 into the coupled equations for $\mathrm{u}$ and $\mathrm{v}$ (equation 3.21) written with an Euler forward time step and rearranging into matrix form yields

$$
\left(\begin{array}{cc}
\lambda-1+k \Delta t & -f \Delta t \\
f \Delta t & \lambda-1+k \Delta t
\end{array}\right)\left(\begin{array}{c}
u^{n} \\
v^{n}
\end{array}\right)=0=\mathbf{A} \vec{u}
$$

To have a solution to the above matrix equation, the determinant of $\mathbf{A}$ must be zero. This gives us a characteristic equation for $\lambda$

$$
\lambda \lambda^{*}=(1-k \Delta t)^{2}+f^{2} \Delta t^{2}
$$

where $\lambda^{*}$ is the complex conjugate of $\lambda$. We see from this equation that the Coriolis term is linearly unstable but the system as a whole can be stablized by the friction term as long as

$$
(1-k \Delta t)^{2}+f^{2} \Delta t^{2} \leq 1
$$

Rearranging, this requires that

$$
\Delta t \leq \frac{2 k}{k^{2}+f^{2}}
$$

The flow is unstable as $k \rightarrow 0$. Because we recognize that the Coriolis term is unstable we use a very small time step in the model calculations. We successively decrease the time step until there is no visible difference in the solution. For the Mediterranean, we find that for time steps below 100 seconds the solutions are indistinguishable. In general, the error arising from this very primitive numerical scheme remains small because the total integration time is only about 10 days.

Given that the friction term is stabilizing our numerical scheme, we need to examine the effect of $\mathrm{k}$ more closely. We rearrange equation 3.24 again and plug in 


\begin{tabular}{|c|c|}
\hline \multicolumn{2}{|c|}{ Initial Parameters } \\
\hline$|\vec{u}|_{o}$ & $0.61 \mathrm{~m} / \mathrm{s}$ \\
$h_{o}$ & $123 \mathrm{~m}$ \\
$W_{\circ}$ & $10 \mathrm{~km}$ \\
$T_{o}$ & $13.2^{\circ} \mathrm{C}$ \\
$S_{o}$ & 37.75 \\
$Q_{o}$, initial transport & $0.75 \mathrm{~Sv}$ \\
starting latitude & $35^{\circ} 54^{\prime}$ \\
starting longitude & $-6^{\circ} 20^{\prime}$ \\
initial angle from due east & $210^{\circ}$ \\
$f$, Coriolis parameter & $8.5 \cdot 10^{-5}$ \\
$g$ & $9.8 \mathrm{~m} / \mathrm{s}^{2}$ \\
$c_{d}$, drag coefficient & $3.0 \cdot 10^{-3}$ \\
$\Delta t$ & $100 \mathrm{~s}$ \\
resolution of topography & 5 minutes \\
\hline
\end{tabular}

Table 3.1: Initial parameters for model.

the specific parameters used in this experiment (given in table 3.1. Given $c_{d}=0.003$, $h=100 \mathrm{~m},|\vec{u}| \approx \mathrm{U}$ then $k \approx 10^{-5} \mathrm{U}$. With $\Delta t=100 \mathrm{sec}$ and $f \approx 10^{-4}$, then the scheme is stable if (rearranging equation 3.24)

$$
\begin{gathered}
k(2-k \Delta t) \geq \Delta t f^{2} \\
\text { or } \\
10^{-5} \mathrm{U}\left(2-10^{-5} \mathrm{U} 10^{2}\right) \geq 10^{2} 10^{-8}
\end{gathered}
$$

We find that we must have $U \geq 0.05 \mathrm{~m} / \mathrm{s}$ for the scheme to remain stable. If $U \approx 0.05$ then the scheme is nearly neutral in which case all of the friction will go to stablize the numerical scheme (note that we physically expect a decay in the solution in the presence of friction, but in this case, the friction is only large enough to remove the growth of the solution from the numerical error in the Coriolis term). On average in the Mediterranean outflow region, $\mathrm{U} \approx 0.5 \mathrm{~m} / \mathrm{s}$. Then for our simulation, we expect about $10 \%$ of the friction term will go to stabilizing the Coriolis term. 


\subsection{Developing intuition: simple analytical solu- tions}

To gain insight into the numerical model, first consider a simplified form of the model equations. Entrainment and variations in height are neglected and a linear friction law is used. The governing equations below a passive upper layer are then

$$
\begin{aligned}
& \frac{d u}{d t}=f v-J u \\
& \frac{d v}{d t}=g^{\prime} \alpha-f u-J v
\end{aligned}
$$

where $\mathrm{J}$ is the friction coefficient, and $g^{\prime}=g \rho^{\prime} / \rho_{0}$ where $\rho^{\prime}$ is the density difference with the uniform layer above. The bottom topography slopes down to the north along the $\mathrm{y}$-axis and has a constant slope, $\alpha$. The $\mathrm{x}$-axis is aligned along the slope.

The solution to this set of equations can be easily obtained by defining $q=u+i v$. We find

$$
\begin{aligned}
(u, v) & =(\operatorname{Re}, \operatorname{Im}) q=(\operatorname{Re}, \operatorname{Im})\left[\frac{g^{\prime} \alpha}{f^{2}+J^{2}}(f+i J)\left(1-e^{i G t}\right)\right] \\
G & =-f+i J
\end{aligned}
$$

where we have assumed that at $t=0, u, v=0$.

\section{Limits of equation 3.27}

It is very instructive to note the limits of this solution. First note that as $\mathrm{J} \rightarrow 0$, we retrieve

$$
\begin{aligned}
& u=\frac{g^{\prime} \alpha}{f}(1-\cos f t) \\
& v=\frac{g^{\prime} \alpha}{f} \sin f t
\end{aligned}
$$


which is the cycloid solution for particle motion on a slope (Knauss, 1978). As $t \rightarrow$ $0, u \sim 0$ and $v \sim g^{\prime} \alpha t$. A particle initially accelerates from rest down the slope. The time average of this solution is $u_{g} \sim g^{\prime} \alpha / f$ and $v_{g} \sim 0$ and represents the steady geostrophic flow along the slope following isobaths. Note that $g^{\prime} \alpha / f$ is also the speed at which an isolated cold eddy will translate along a sloping bottom (Nof, 1983, 1990).

As $f \rightarrow 0$ in equation 3.27 , we retrieve

$$
\begin{aligned}
u & =0 \\
v & =\frac{g^{\prime} \alpha}{J}\left(1-e^{-J t}\right),
\end{aligned}
$$

the nonrotating 'uniform flow' solution (Chow, 1959). As $t \rightarrow 0, v \sim g^{\prime} \alpha t$. A particle initially accelerates downslope at the same speed as in the frictionless limit. As $\mathrm{t} \rightarrow \infty$, the frictional steady velocity becomes $u_{f} \sim 0$ and $v_{f} \sim g^{\prime} \alpha / J$. Friction dissipates the velocity which is continually supplied by the decrease in potential energy as the particle moves down the slope.

Equation 3.27 implies a steady (or particular solution) which we define as

$$
u_{s}=\frac{g^{\prime} \alpha f}{f^{2}+J^{2}} \quad \text { and } \quad v_{s}=\frac{g^{\prime} \alpha J}{f^{2}+J^{2}} .
$$

The ratio of $v_{s} / u_{s}=J / f$. This ratio describes the angle the flow makes relative to the isobaths (the $\mathrm{x}$ axis) and describes the regime of the flow: inviscid to frictionally dominated. If we scale $u, v$ with these steady scales we find that $J^{2} / f^{2}$ also represents the Ekman number, E.

To compare with the plume model presented in section 3.1, we note the $J \sim$ $c_{d} U / H$. For the Mediterranean outflow we have $c_{d} \sim 3 \cdot 10^{-3}, U \sim 1 / 3 \mathrm{~m} \mathrm{~s}^{-1}, \mathrm{H}$ $\sim 100 \mathrm{~m}$, and $f \sim 10^{-4} \mathrm{~s}^{-1}$. Assuming these scales, $J / f$ is approximately $1 / 10$. Near the Strait, where the flow speeds reach $1 \mathrm{~m} / \mathrm{s}, J / f \sim 1 / 3$. Therefore, assuming an average value of $J / f \sim 2 / 10$, we expect that the flow will be approximately geostrophic and will descend isobaths on average at an angle, $\beta=11.3^{\circ}$, where 
$\tan (\beta) \sim J / f$. Note that this is equivalent to descending $700 \mathrm{~m}$ in the $350 \mathrm{~km}$ path of the outflow through the Gulf of Cadiz, very close to the expected descent of the outflow (assuming that $\delta z \sim \alpha \delta x J / f$, with $\alpha \sim 0.01$ ).

\section{Path, frequency, and wavelength of cycloid motion}

The particle trajectory or path can be found by defining $\bar{X}=\int q d t$, where $\bar{X}=$ $(x-x(t=0))+i(y-y(t=0))$. Integrating, we find

$$
\bar{X}(t)=\frac{g^{\prime} \alpha}{J^{2}+f^{2}}(f+i J)\left[t+\frac{J-i f}{f^{2}+J^{2}} e^{i t(-f+i J)}\right]
$$

As $\mathrm{J} \rightarrow 0$, we find $x-x(t=0)=\frac{g^{\prime} \alpha}{f^{2}}(f t-\sin f t)$ and $y-y(t=0)=-\frac{g^{\prime} \alpha}{f^{2}} \cos f t$. In this frictionless limit, a particle translates in the $\mathrm{x}$ direction and moves up and down the slope but over a period the particle returns to its starting position on the slope (i.e. it returns to $y(t=0))$.

To find the wavelength and frequency of the oscillations in the solution we first note that

$$
|\vec{u}|^{2}=\frac{\left(g^{\prime} \alpha\right)^{2}}{J^{2}+f^{2}}\left[1+e^{-2 J t}-2 e^{-J t} \cos f t\right] .
$$

The speed, $|\vec{u}|$ reaches extrema when its first derivative vanishes, namely

$$
J e^{-J t}=f \sin f t+J \cos f t .
$$

As $\mathrm{J} \rightarrow 0$ this simplifies to $\sin (\mathrm{ft})=0$ (as expected). For frictional flow, as $\mathrm{t} \rightarrow \infty$ the extrema occur at $\tan (\mathrm{ft})=-\mathrm{J} / \mathrm{f}$. Thus the extrema in speed get shifted earlier in time as $\mathrm{J} / \mathrm{f}$ increases. The roots of equation 3.33 can be seen graphically in figure 3.4 as the intersection between the two curves, $\left(\mathrm{J} \mathrm{e}^{-J t}\right)$, and $(\mathrm{f} \sin (\mathrm{ft})+\mathrm{J} \cos (\mathrm{ft}))$. This figure shows that the effect of friction is to initially vary the period between the highest and lowest velocity. The second derivative of $|\vec{u}|$ (not shown) reveals that the time from the minimum to the maximum speed is shorter than the time from the maximum 


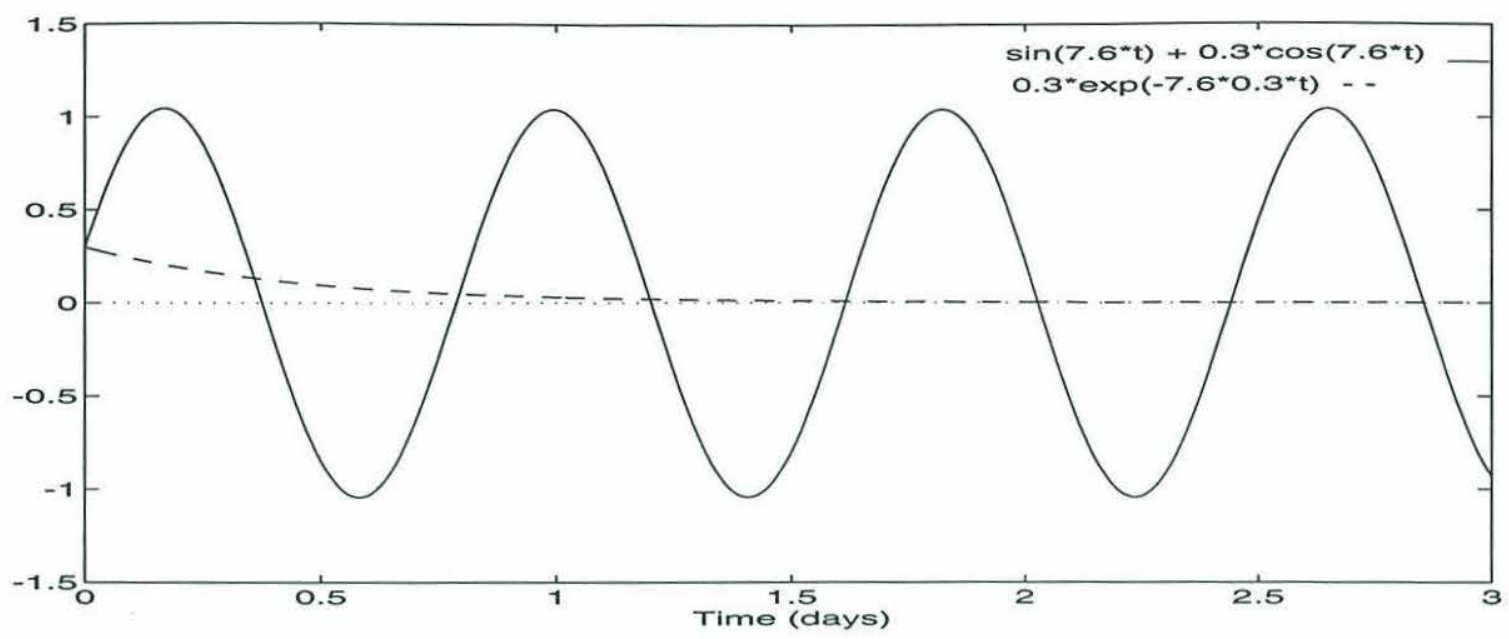

Figure 3.4: The effect of friction in determining the period of a cycloid motion is to initially offset the minima and maxima in velocity. The extrema in the speed of a cycloid slowed by friction is given by the roots of equation 3.33. The roots are visualized here as the intersection of the two curves, $\mathrm{J} e^{-J t}$, and $\mathrm{f} \sin (\mathrm{ft})+\mathrm{J} \cos (\mathrm{ft}) . \mathrm{J} / \mathrm{f}=0.3$, and $2 \pi / \mathrm{f}$ $=.83$ days.

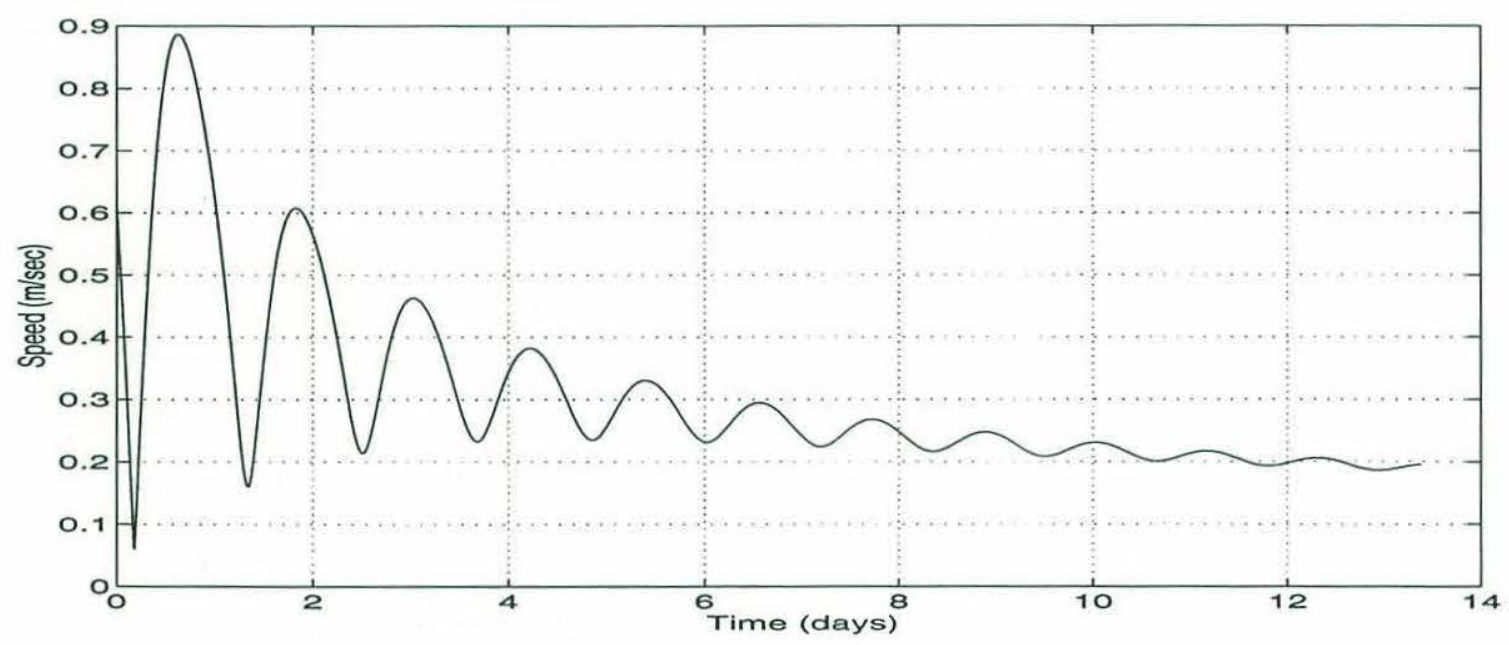

Figure 3.5: Speed of a cycloid trajectory modified by friction. The speed decreases in time, damped by friction $\left(\mathrm{J}=5 \cdot 10^{-6}\right)$. The maxima and minima in velocity are slightly offset (i.e. not $\mathrm{T} / 2$ apart). The flow decelerates faster than it accelerates.

to the minimum speed (i.e. a particle takes less time to accelerate than decelerate). The velocity decay and phase shift between maximum and minimum speed is shown graphically in figure 3.5 . 
Neglecting the subtle variations arising from the presence of friction, the peak to peak period for this model is approximately $T \sim \frac{2 \pi}{f}$. The length scale at any point along the path is given by the $\int|\vec{u}| d t$ over a full period. A closed form of this integral is not apparent, so we note instead that in the limit of $t \rightarrow \infty$, the steady velocity has the magnitude $\left|\vec{u}_{s}\right| \sim \frac{g^{\prime} \alpha}{\left(f^{2}+J^{2}\right)^{1 / 2}}$. The steady wavelength of these oscillations up and down the slope is then

$$
L=\frac{2 \pi g^{\prime} \alpha}{f\left(f^{2}+J^{2}\right)^{1 / 2}} .
$$

The effect of stratification

With a uniform upper layer, we found that the period of the inertial oscillations is $2 \pi / f$ and the length scale is $\frac{2 \pi g^{\prime} \alpha}{f\left(f^{2}+J^{2}\right)^{1 / 2}}$. The model presented in section 3.3 moves through a stratified environment so that the density anomaly and hence $g^{\prime}$ depends on the vertical position of the particle. To consider the effect of the ambient density, we note that for a constant background stratification, $N^{2}$, the reduced gravity, $g^{\prime}=g \rho^{\prime} / \rho_{o}-N^{2} \alpha y$, where y is the axis normal to the topography (i.e. positive upslope) and $\left(\rho^{\prime}+\rho_{o}\right)$ is the density of the modeled layer. Neglecting friction, the model equations become

$$
\begin{aligned}
& \frac{d^{2} x}{d t^{2}}=f \frac{d y}{d t} \\
& \frac{d^{2} y}{d t^{2}}=g \rho^{\prime} \alpha / \rho_{\circ}-N^{2} \alpha^{2} y-f \frac{d x}{d t}
\end{aligned}
$$

Rewriting as one equation for $\mathrm{y}$, this becomes

$$
\frac{d^{3} y}{d t^{3}}+\left(f^{2}+N^{2} \alpha^{2}\right) \frac{d y}{d t}=0
$$

Assuming that $y=y_{0} e^{i \omega t}$, we can solve for the frequency,

$$
\omega^{2}=f^{2}+N^{2} \alpha^{2}
$$


Note that this is very similar to the frequency of internal waves with constant $N^{2}$. The frequency for internal waves can be given by $\omega^{2}=f^{2} \sin ^{2}(\theta)+N^{2} \cos ^{2}(\theta)$, where $\theta$ is the angle the wavenumber vector makes to the horizontal axis. For inertial oscillations (the only waves present in the previous section), $\theta=\pi / 2$. With the introduction of stratification, we have effectively defined $\theta=\pi / 2+\alpha$, where $\alpha$ is the bottom slope. For small $\alpha$ we approximately find $\omega^{2}=f^{2}\left(1-\alpha^{2}\right)+N^{2} \alpha^{2}$ which is approximately equation 3.37 except that in internal waves, the Coriolis term is reduced by ( 1 $\left.\alpha^{2}\right)$. Therefore, we expect the waves present in the model will have a slightly higher frequency than internal waves with the same angle of inclination (from vertical). 


\subsection{Mediterranean outflow simulation and com- parison with the 1988 Cadiz Expedition data}

\subsubsection{Overview}

The model is initialized to the west of Camarinal Sill, the main sill in the Strait of Gibraltar, with the initial conditions and location listed in table 3.1. The model is then integrated forward in time following the flow. The model predicts the evolution of $\vec{u}, T, S, h, W$, and $\vec{x}$ along the path of the outflow, which follows the northern continental slope in the Gulf of Cadiz, shown in figure 3.6. These model variables, the bulk Froude number and the simulated outflow volume transport are shown in figures $3.7,3.8,3.9$, and 3.10 . The overall trend of the simulated outflow solution corresponds well to the historical observations and the new data obtained in the 1988 Cadiz Expedition and analyzed in chapter 2 (see figure). The rapid decrease of the simulated outflow temperature and salinity indicates very localized, vigorous entrainment of overlying fresh and cold North Atlantic Central Water (NACW) into the outflow layer, occurring in the first $50 \mathrm{~km}$ (see figure 3.11).

The path of the simulated outflow shows that the flow turns to the right by about $90^{\circ}$ after it exits the strait in the first half day of integration (along the path symbols are plotted marking every half day). Also in figure 3.6, is the predicted density of the simulated outflow as it moves through the Gulf shown with the ambient density profile. Note that the density of the outflow corresponds to different horizontal positions. The outflow begins near 300 meters depth with density, $\sigma_{\theta}=28.5$ and descends as it moves through the Gulf entraining ambient water whose density is also shown in figure $3.6 \mathrm{~b}$. The model predicts that the majority of entrainment occurs in the first day of integration (in figure 3.6 symbols are plotted for every half day of integration) where the simulated outflow descends from $300 \mathrm{~m}$ to $600 \mathrm{~m}$. After that, 
a.

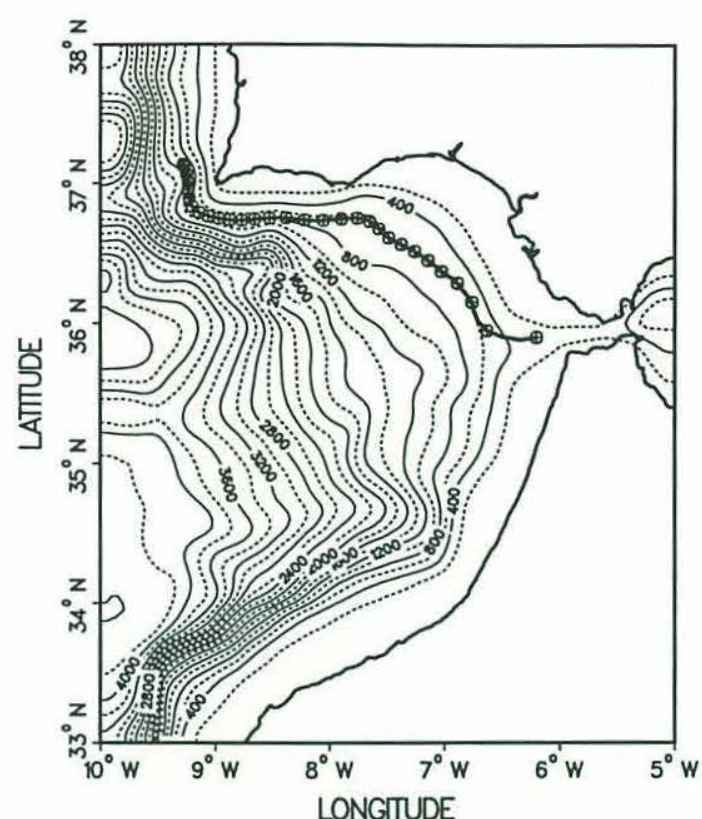

b.

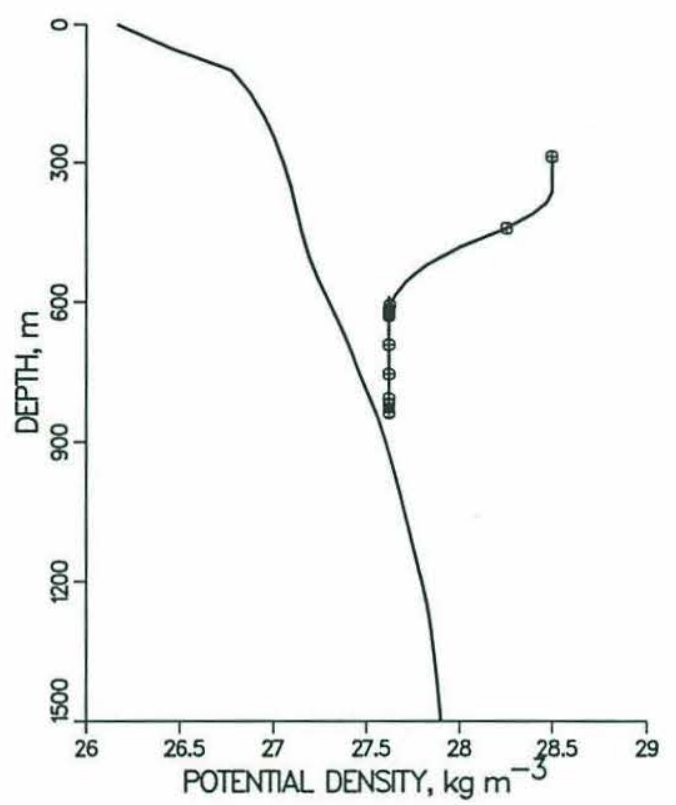

Figure 3.6: a) Gulf of Cadiz bathymetry and simulated outflow plume path. Markers representing every half day of model integration are placed along the plume's path. Note that the total transit time for a particle to move through the Gulf of Cadiz is only 10 days. b) The density of the simulated outflow layer as it progresses through the Gulf of Cadiz (solid line with symbols corresponding to half day intervals). The depth of the outflow is taken to be the mid-column depth, appropriate for calculation of the buoyancy forcing and indicated in the schematic figure 3.1. The dashed line is the ambient density profile taken from the 1988 Cadiz Expedition station 135. This profile is assumed to represent the background density profile throughout the Gulf of Cadiz. This and the following figures 3.73.11 are similar to the results of Price and Baringer (1993). They differ in that the model has the refined initial conditions suggested by the GofCExp data to which the model is also compared with in these figures. The most substantial change is the initial transport which is reduced from $1 \mathrm{~Sv}$ used in Price and Baringer (1993), to the more recently calculated value of $0.7 \mathrm{~Sv}$ given in Chapter 2 (and consistent with the recent current measurements of Bryden, Candela and Kinder, 1993).

the outflow descends slowly without changing density. The integration is stopped after $350 \mathrm{~km}$, with the middle of the simulated outflow near $850 \mathrm{~m}$. The simulated outflow density at the end of run correspond to a depth of about $1000 \mathrm{~m}$ in the ambient density profile. This depth is well within the range expected for the outflow to enter the open ocean. 

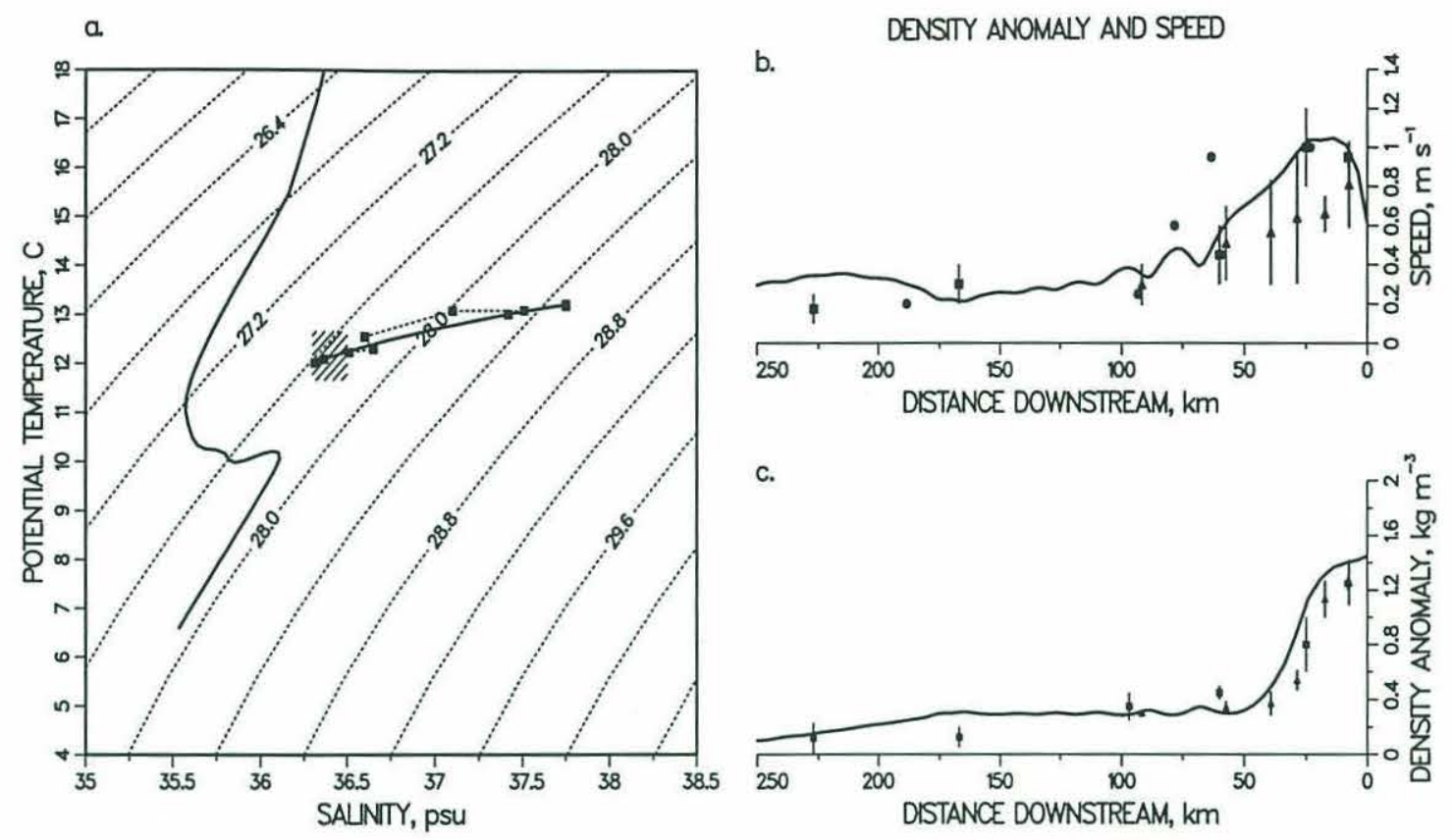

Figure 3.7: a) Temperature versus salinity diagram showing the evolution of the outflow properties (heavy solid line). Symbols mark every $25 \mathrm{~km}$ of the simulated outflow path. The ambient stratification is shown with a solid line. Dashed contours are potential density. The dashed line with rectangular symbols represents the GofCExp data (as alternatively shown in figure 3.8). b) Current speed and c) density anomaly along the axis of the plume. Density anomaly is the density difference between the plume and the surrounding waters. The 1988 Cadiz Expedition data is shown with error bars and open triangular symbols. Data with error bars and solid square symbols are from Smith (1975), data without error bars is from Heezen and Johnson (1969).

\subsubsection{Property Evolution}

The plume model integration begins just east of our Section A, where the topographic slope is weak (on the order of $10^{-3}$, see figure $3.10 \mathrm{~d}$ ). Initially, the flow is confined to a channel with a specified width. The initial velocity is $0.61 \mathrm{~m} / \mathrm{s}$ and the total transport is $0.76 \mathrm{~Sv}$. Note that this is approximately the total transport predicted at the section A from the GofCExp. The initial Froude number is well below one and no mixing occurs during the first $10 \mathrm{~km}$. 


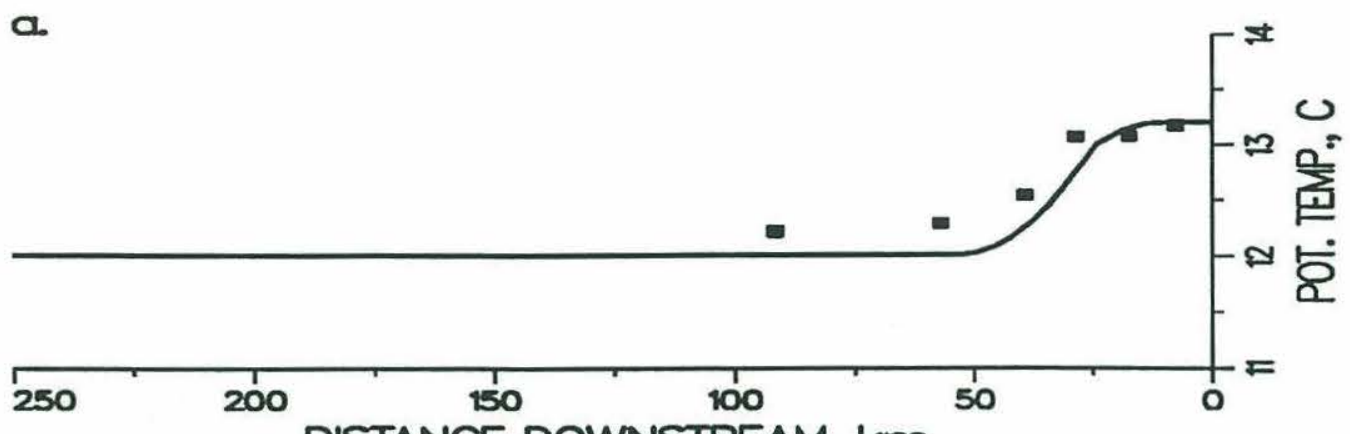

DISTANCE DOWNSTREAM, km
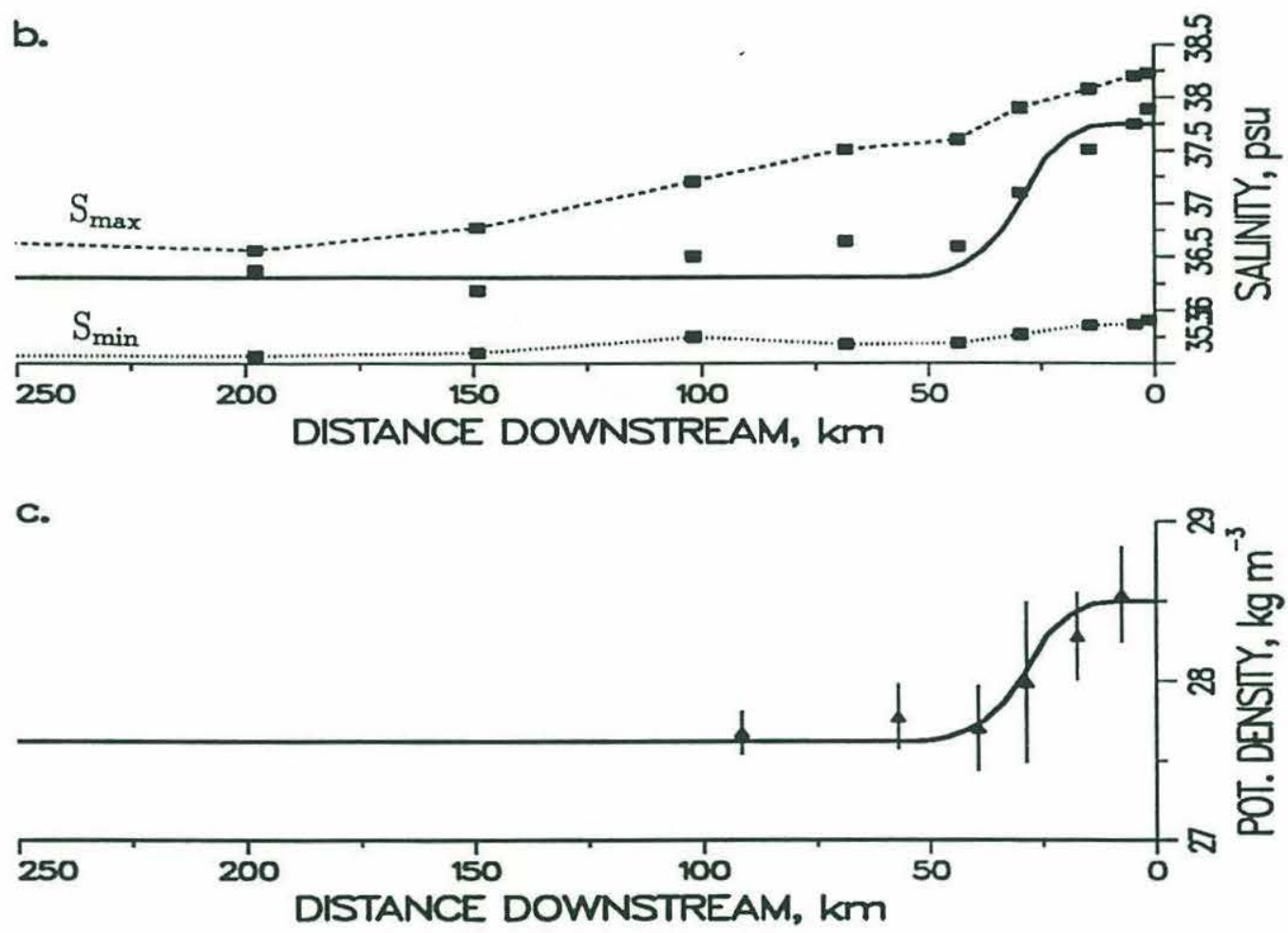

Figure 3.8: Downstream evolution of simulated outflow temperature, salinity and density. a) Potential temperature of the outflow. GofCExp data shown in square symbols (calculated as a velocity weighted temperature similar to salinity (see chapter 2). b) Salinity of the outflow. GofCExp outflow salinity shown as square symbols (see chapter 2 for calculation details). The maximum salinity within the outflow (from the GofCExp data) shown with a dashed line. The minimum salinity representing NACW taken from the GofCExp data shown with a dotted line. c) Potential density of the simulated outflow. The $1988 \mathrm{Cadiz}$ Expedition data is shown with error bars and open triangular symbols (calculated as a velocity weighted potential density in the same manner as the salinity. The error bars represent \pm one standard deviation). 

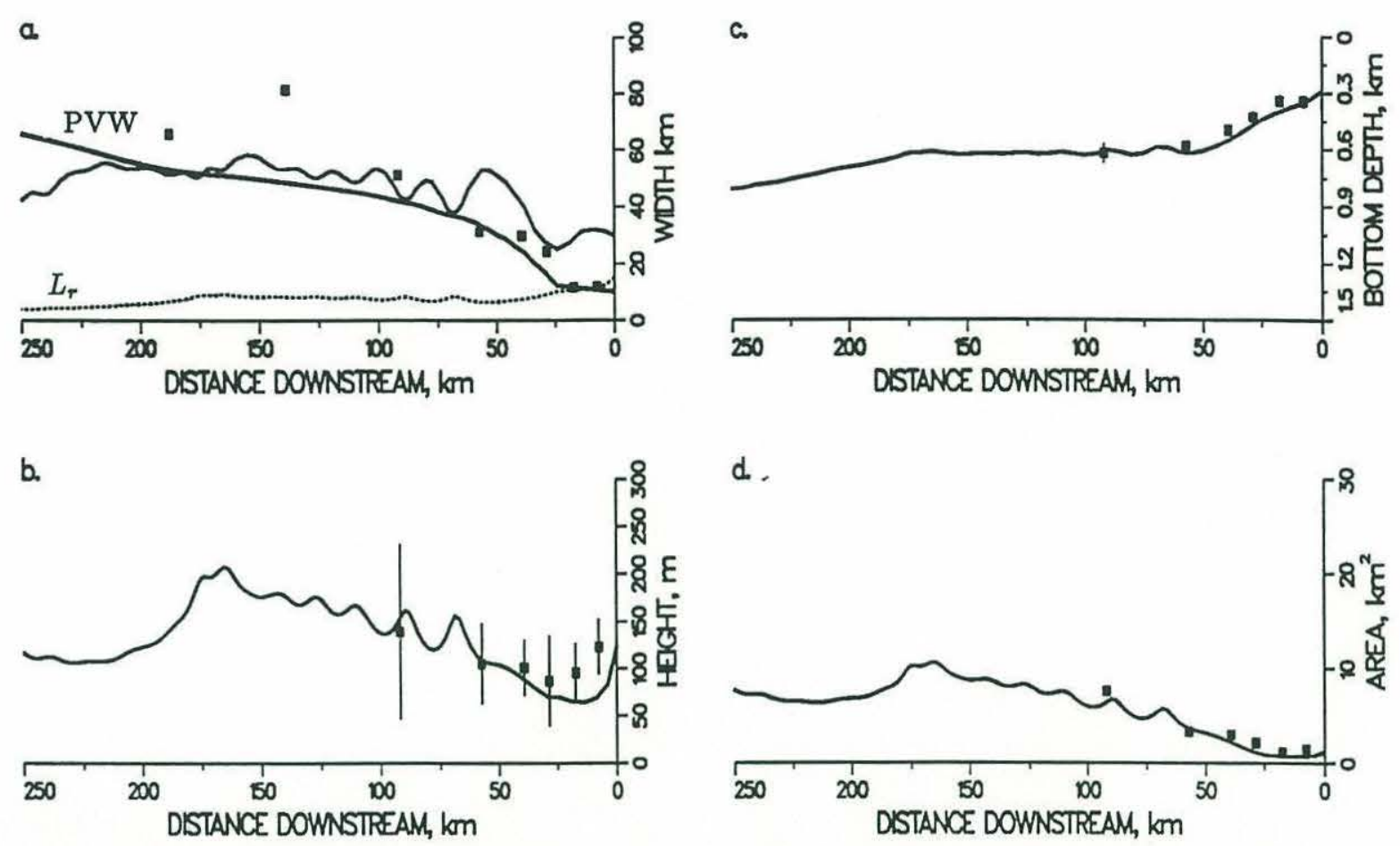

Figure 3.9: Downstream evolution of the simulated outflow $h, W$, depth, and area. a) Width of layer (solid line). GofCExp data width defined as the maximum extent of the 36.25 isohaline at each section (square symbols). The internal Rossby radius is shown with the dashed line and labeled $L_{r}$. Another esitmate for the width which assumes that the potential vorticity is conserved is calculated passively in this simulation and is shown with the heavy solid line labeled PVW (see section 3.5). b) Height of the simulated outflow layer. The 1988 Cadiz Expedition average height is shown with error bars representing \pm one standard deviation. c) Depth of the middle of the layer (solid line). GofCExp depth calculated as the center of density anomaly (square symbols). Error bars represent \pm one standard deviation from other possible methods. d) Total cross sectional area of layer. GofCExp data shown with square symbols.

As the flow leaves the continental shelf near $6^{\circ} 25^{\prime}$, the increase in bottom slope together with the large initial density anomaly forces the simulated outflow to accelerate. The slope begins to increase to values above $10^{-2}$ and the average speed increases above $1.0 \mathrm{~m} / \mathrm{s}$. After the first $25 \mathrm{~km}$, the layer is allowed to freely spread subject to equations 3.18 and 3.19. The increased velocities lead to a very 

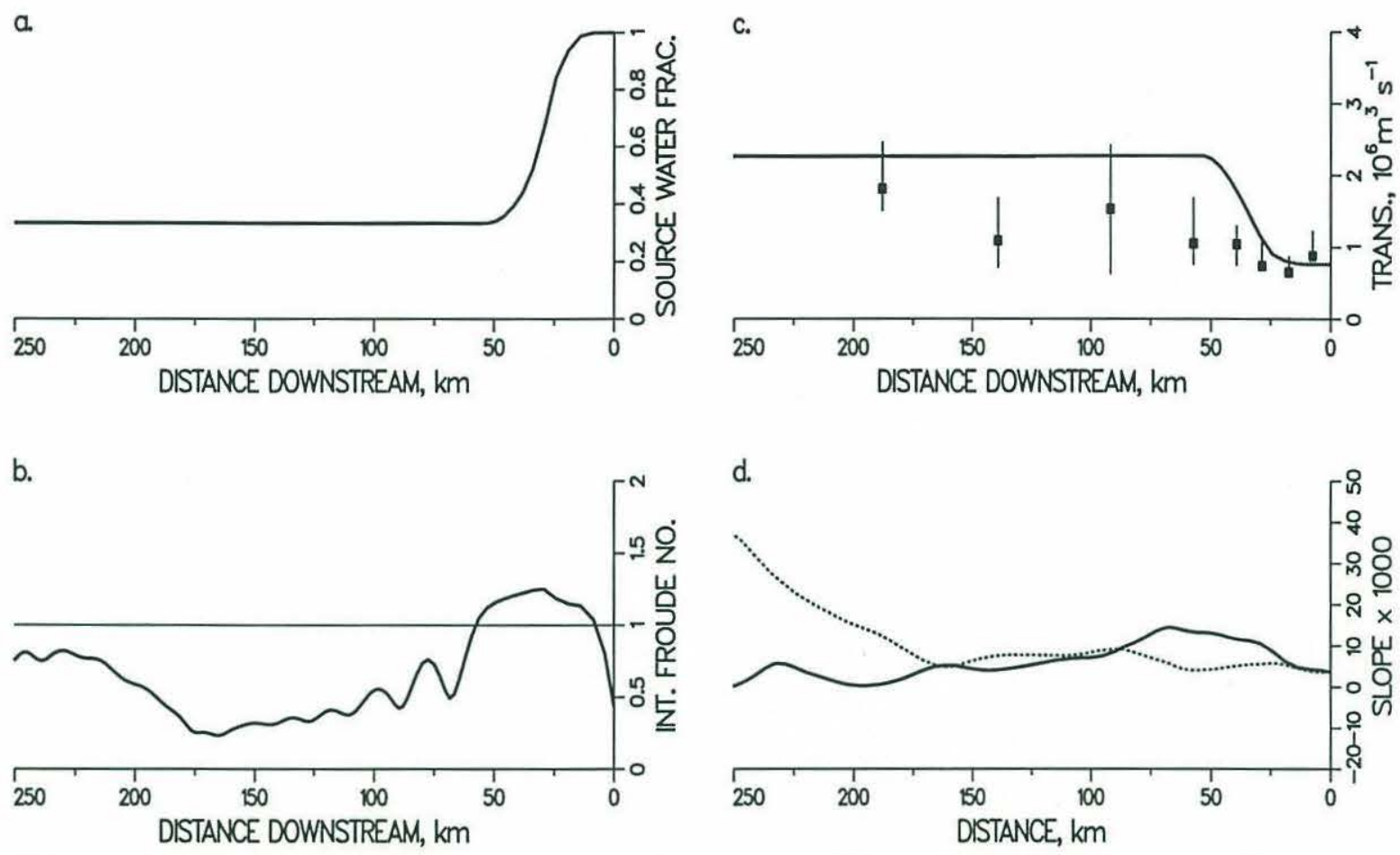

Figure 3.10: Downstream evolution of the simulated outflow $F r_{b}$, source water fraction, transport, and bottom slope. a) fraction of source water present. The initial conditions are considered to be $100 \%$ source water even though we initialize the model with slightly mixed water and 'pure' Mediterranean source water would have salinities as high as 38.4 pss. b) Froude number of the layer (solid line). The solid line marks Froude numbers greater than one indicating where mixing increases rapidly. c) total volume transport. Square symbols represent the transports from the XCP velocities from the GofCExp using the temperature minimum as the zero reference level. Error bars represent the transport range based on isopycnal reference levels in the range of densities at the temperature minimum. d) slope of the bottom topography. The downstream component is shown with the solid line. The cross slope component is shown as a dashed line.

large spreading rate where the Ekman number, E, reaches a maximum of $0.48^{3}$. The fast spreading coupled with the increased speed causes the simulated outflow layer to thin (by continuity). The Froude number is raised above one (shown in figure 3.10b), initiating strong mixing. The potential temperature and salinity decrease from the initial values of $13.2^{\circ} \mathrm{C}$ and 37.75 pss to $12.01^{\circ} \mathrm{C}$ and 36.31 pss respectively, in the

\footnotetext{
${ }^{3}$ Note that this implies that $v \sim u$ and not $v \ll u$ as Smith (1975) requires.
} 


\section{ENTRAINMENT RATE}

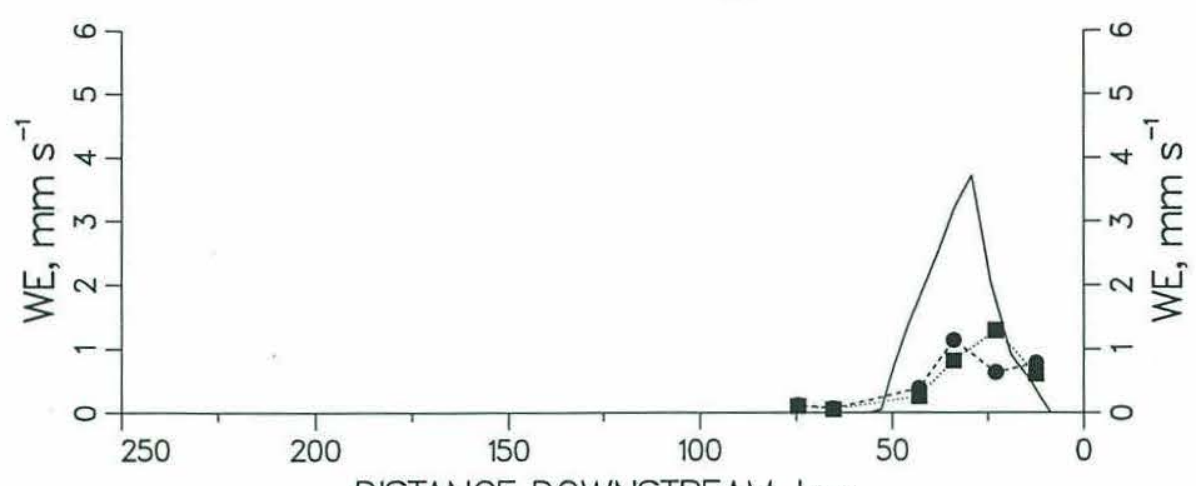

DISTANCE DOWNSTREAM, $\mathrm{km}$

Figure 3.11: Entrainment rate of the simulated outflow (solid line). Two different entrainment estimates discussed in chapter two are plotted: the entrainment from the salt equation is shown with square symbols and dotted line, the entrainment estimated from the increase in transport is shown with round symbols and dashed line.

first $50 \mathrm{~km}$ (figure $3.8 \mathrm{a}$ and $\mathrm{b}$ ). The initial mixing stage is shut off by an inertial oscillation that decelerates the plume by forcing it up the slope (see discussion in the next section).

No further mixing occurs as the layer descends and transits the Gulf of Cadiz. Near $175 \mathrm{~km}$ downstream, the slope increases rapidly to values in excess of $3 \cdot 10^{-2}$ and the flow accelerates again to speeds near $0.35 \mathrm{~m} / \mathrm{s}$. This acceleration is accompanied by an increase bottom drag that allows the flow to cross isobaths and slide more rapidly down the topography. The width also increases more rapidly and the outflow thins suddenly. Higher bulk Froude numbers are predicted from this increase in speed and thinning of the layer, but the Froude number remains below one and no mixing is initiated. The density anomaly slowly decreases not because of entrainment, but because the outflow is descending into a stratified background. We will see later that although the final properties of the simulated outflow are fairly insensitive to the initial conditions and external parameters, there can be a second mixing event as the flow accelerates in this region.

The simulated outflow leaves the Gulf of Cadiz having increased its transport by almost a factor of three (see source water fraction in figure $3.10 \mathrm{a}$ ). The plume equi- 
librates at a depth of about $1000 \mathrm{~m}$ with a total transport of $2.2 \mathrm{~Sv}$, having entrained $1.45 \mathrm{~Sv}$ of fresh and cool North Atlantic Central Water. The model integration is terminated after the flow has left the Gulf of Cadiz and turned northward along the coast of Portugal (figure 3.6).

\subsubsection{Dynamical Balances in the Model}

For insight into the dynamical processes controlling the flow, it is convenient to rotate the momentum equations into coordinates aligned with the flow. The alongstream direction is defined as the instantaneous direction of the flow while the crossstream direction is perpendicular to the flow path. Figure 3.1 shows the force balances. We see that in this reference frame the bottom and entrainment stress will only appear in the along-stream momentum equation. The Coriolis term must by definition only appear in the cross-stream momentum balance because $\vec{u} \cdot \vec{f} \times \vec{u} \equiv 0$. The momentum balances in the along-stream and cross-stream directions are shown in figure 3.12 . We define each term in the momentum equation as indicated below

$$
\underbrace{\rho_{0} h \vec{u} \cdot \nabla \vec{u}}_{\text {acceleration }}=\underbrace{g \delta \rho h \nabla D}_{\text {buoyancy }}-\underbrace{\rho_{0} h \vec{f} \times \vec{u}}_{\text {Coriolis }}-\underbrace{\overrightarrow{\tau_{b}}}_{\text {bottom stress }}-\underbrace{\overrightarrow{\tau_{e}}}_{\text {entrainment stress }}
$$

\section{Cross-stream Momentum}

The cross-stream momentum balance is shown in figure $3.12 \mathrm{a}$ and as expected, represents a nearly geostrophic balance between the cross-stream buoyancy and the Coriolis acceleration. The residual of the cross-stream momentum (the heavy solid line in figure $3.12 \mathrm{a}$ ), represents the acceleration or inertial terms. In this curvilinear coordinate system, the cross-stream acceleration indicates curvature in the flow. If $\beta$ is the angle of a particle trajectory relative to the local tangent to the trajectory in 


\section{MOMENTUM BALANCE}

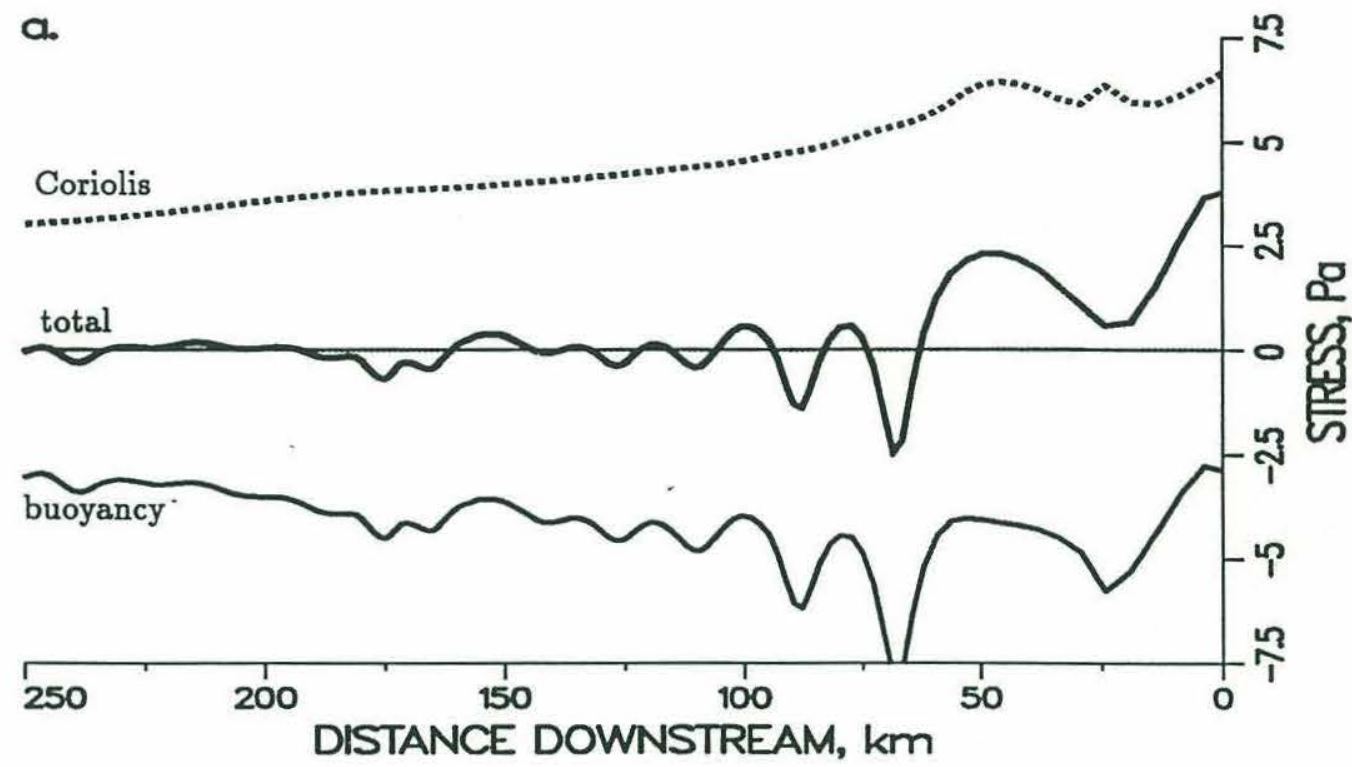

b.

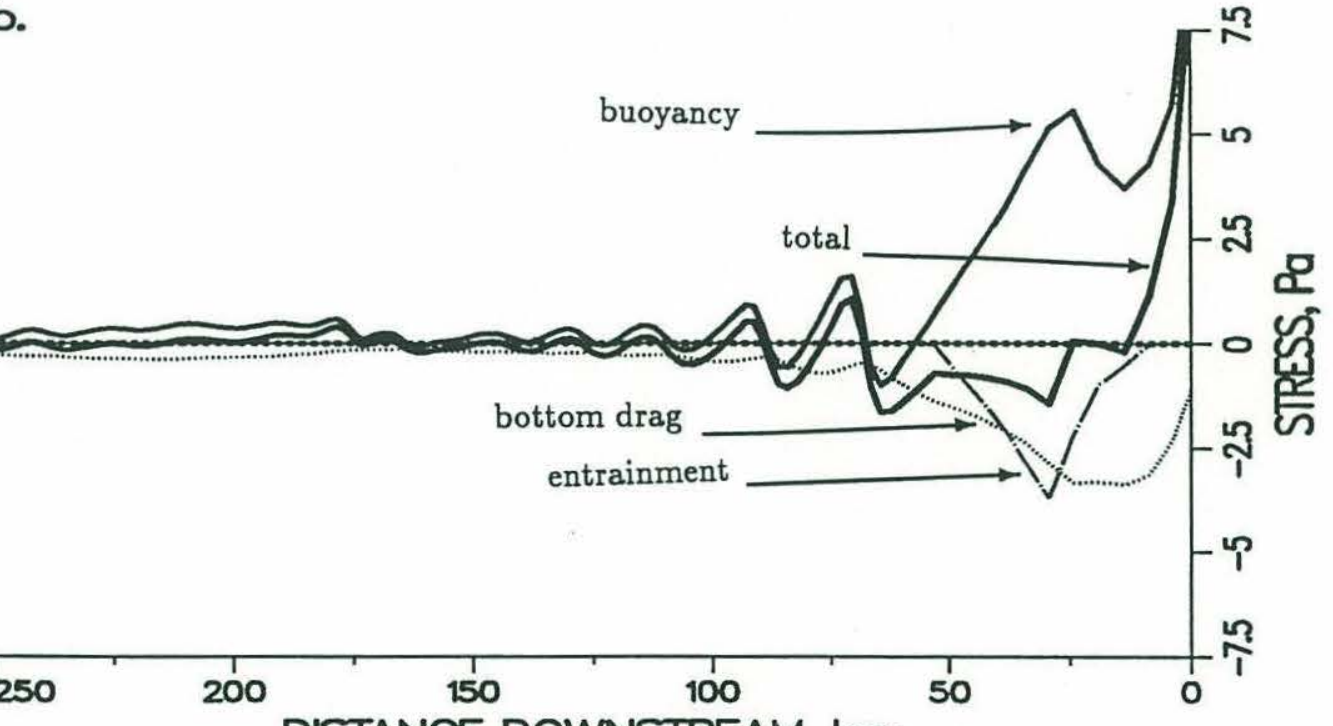
DISTANCE DOWNSTREAM, km

Figure 3.12: Momentum balance in the a) cross-stream and b) along-stream directions as a function of downstream distance. The light solid line is the buoyancy; the dashed line is the Coriolis force; the chain-dotted line is the entrainment stress; the dotted line is the bottom drag; and the heavy line is the sum of all the above (i.e. the resulting force on the plume). 
this curvilinear coordinate system, the cross-stream acceleration term can be written

as $\rho_{o} h V^{2} \frac{d \beta}{d s}$, where $V$ is the speed in the downstream direction, $s$ (see chapter 2.6). The residual between the buoyancy and Coriolis forces represents a turning of the flow to the right or left for positive and negative values respectively.

Figure $3.12 \mathrm{a}$ shows that the flow turns to the right during the first $65 \mathrm{~km}$ downstream (positive 'total' stress in the cross-stream momentum balance). After this initial turn, the flow oscillates to the right and left in a steadily decaying inertial oscillation. We can define a Rossby number, $R_{o}$, for the flow as a ratio of the inertial curvature term to the Coriolis term. Within the first $65 \mathrm{~km}, R_{o}$ is quite large, reaching a maximum of 0.6 initially with an average value close to $\frac{1}{3}$. Further downstream, the cross-stream inertia of the flow is much less important: $R_{o}$ is less than 0.1. Although the initial descent of the overflow includes strong acceleration, further downstream the layer is nearly in geostrophic balance with acceleration having less than a $10 \%$ effect on the flow.

\section{Along-stream Momentum}

The along-stream force balance is in some ways more interesting since it shows the changes in speed. The residual of the downstream force balance (heavy solid line in figure 3.12) represents the acceleration of the along-stream velocity. Positive (negative) residual indicates acceleration (deceleration) of the layer. Initially, the flow is accelerated when it first encounters the continental slope because of the large buoyancy forcing (up to $5 \mathrm{~Pa}$, figure $3.12 \mathrm{~b}$ ). As the flow accelerates, the bottom stress increases rapidly (see also figure 3.2 ). Notice that the bottom stress reaches a maximum in excess of $3.0 \mathrm{~Pa}$, as the acceleration ceases near $15 \mathrm{~km}$. As the flow accelerates, the Froude number increases (figure $3.10 \mathrm{~b}$ ) which initiates strong mixing (figure 3.11 ) and hence an entrainment stress (see figure 3.2 ). Together, the bottom 
stress and entrainment stress largely balance the buoyancy forcing within the first 60 $\mathrm{km}$.

\section{Inertial Oscillations}

Further downstream, the flow accelerates and decelerates in an steadily decreasing inertial oscillation. The oscillations in the flow give a cycloidal particle trajectory under the influence of rotation and buoyancy (discussed in section 3.2). In the inviscid cycloid problem on a constant slope, the flow is deflected to the right, up the slope to its original depth and decelerates. It then turns left and accelerates down the slope again. With friction, the mean particle motion continues down the slope and the left turn in the flow becomes less abrupt. Friction steadily decreases the amplitude of the inertial oscillations. Downstream of $180 \mathrm{~km}$, the oscillations are all but absent and the steady along-stream balance is between friction and the along-stream pressure gradient.

Notice that between $15 \mathrm{~km}$ and $75 \mathrm{~km}$ downstream the along-stream flow is decelerated. Near $60 \mathrm{~km}$ downstream, the flow is still turning to the right which eventually leads the flow uphill. The along-stream buoyancy forcing decelerates the flow as it attempts to move uphill in its first broad inertial turn. As the speed is reduced the Froude number decreases and entrainment is shut off. Thus the inertial turn in the flow acts to shut off the entrainment. We will see in the next section, that under different initial conditions buoyancy can accelerate the flow again further downstream at about $175 \mathrm{~km}$ where the topographic slope begins to increase.

From the simple analytical solutions discussed in section 3.2 we estimated the frequency of the inertial oscillations modified by a variable buoyancy should be approximately $\omega^{2}=f^{2}+N^{2} \alpha^{2}$ (equation 3.37). In the model solution we find two regions where the 'wiggles' in the flow are most apparent: between 50 and $150 \mathrm{~km}$ 
and beyond $300 \mathrm{~km}$ (note shown). The model oscillations are very regular in these regions and have periods of 16.1 and 9.1 hours respectively ( 0.67 and 0.38 days). Pure inertial oscillations at this latitude have a period of 19.7 hours (0.82 days), substantially larger than the periods observed. From the ambient density profile used in the model (GofCExp station number 135), $N^{2} \sim 1.2 \cdot 10^{-5} \mathrm{~s}^{-1}$. Given that the slope between 50 and $150 \mathrm{~km}$ is close to 0.015 and that the slope beyond $300 \mathrm{~km}$ is larger than 0.04 , the modified period of the oscillations predicted by the dispersion relation derived in section 3.2 are 17.0 and 10.6 hours ( 0.71 and 0.44 days). The oscillations in the flow therefore appear to be mixed inertial-buoyancy oscillations. The presence of variable stratification and changing topography causes the dominant period of the solution to change as the outflow moves downstream.

\section{Energy Balance}

The downstream evolution of the simulated outflow can be summarized by considering the energy of the system. An energy equation can be obtained in the usual way by adding $u$ times equation 3.10 to $v$ times equation 3.11 . The energy equation is

$$
\underbrace{\frac{d}{d t}\left(\frac{1}{2} \rho_{o}|\vec{u}|^{2}\right)}_{\text {kinetic energy }}=\underbrace{g \delta \rho \vec{u} \cdot \nabla D}_{\text {pressure work }}-\underbrace{\frac{\rho_{o} c_{d}}{h}|\vec{u}|^{3}}_{\text {drag }}-\underbrace{\frac{\rho_{o} w_{e}}{h}|\vec{u}|^{2}}_{\text {entrainment }}-\underbrace{\frac{1}{h} \vec{u} \cdot \nabla\left(\frac{1}{2} g \delta \rho h^{2}\right)}_{\text {internal pressure work }}
$$

Note that the internal pressure work is not explicitly included in the model and is calculated here as a passive diagnostic (see appendix $\mathrm{C}$ for further discussion). This equation can be integrated through time to determine the net work done on the system (i.e. Joules $/ \mathrm{m}^{3}$ ).

Figure 3.13 shows each term in the energy equation. Most of the energy conversion occurs within the first $50 \mathrm{~km}$ that the outflow moves downstream. Pressure 


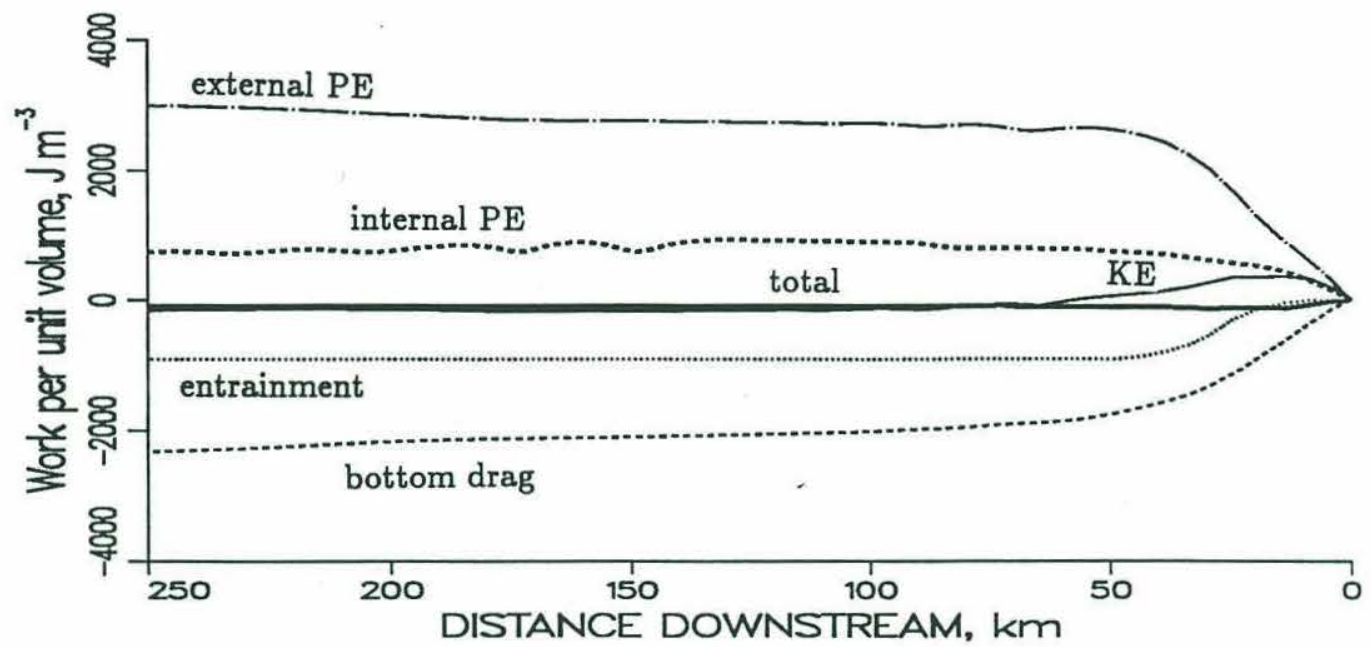

Figure 3.13: Energy balance integrated in time as a function of downstream distance. The chain-dotted line is the external pressure work; the heavy dotted line is the internal pressure work which is not explicitly included in the model; the light solid line is the kinetic energy; the dotted line is the entrainment; the dashed line is the bottom drag; and the heavy line is the sum of all the above except the internal pressure work (i.e. the total change in energy which we expect to be zero).

entrainment stress reaches values as large or larger than the maximum bottom stress (both peak values are near $3 \mathrm{~Pa}$, see figure 12), the net importance of entrainment is only about one third that of bottom stress. The downstream acceleration and turning of the flow were relatively small but still prominent features in the momentum balance. The inertia in the energy equation, however, is even smaller, showing a slight increase in kinetic energy quite rapidly within only $10 \mathrm{~km}$ of the origin and thereafter a gradual decrease to about $75 \mathrm{~km}$ downstream. The internal pressure work (heavy dashed line in figure 13) is not explicitly included in the model and has been calculated here as a passive diagnostic of the model solution. This term, which we have assumed to be small appears as large as the entrainment stress term which we have included. The implications of this necessary and unsatisfying approximation is discussed in more detail in appendix C. In summary, the simulated outflow is forced by pressure work which is dissipated mainly by drag from bottom stress. 
discussed in more detail in appendix C. In summary, the simulated outflow is forced by pressure work which is dissipated mainly by drag from bottom stress.

\subsection{Model Sensitivity}

The robustness of the model solution can be assessed by evaluating the sensitivity to the initial conditions and parameters. Several model experiments have been conducted to determine the sensitivity to variations in initial conditions and internal parameters such as the bottom friction. Figure 3.14 and 3.15 show some of the results of these experiments by plotting the initial conditions against the final equilibrium properties. The dominant balances and the intermittence of entrainment do not change with different initial conditions. When the speed is increased or the bottom drag coefficient is decreased the solution exhibits more vigorous inertial oscillations. A second intermittent mixing stage is also present when the bottom stress is reduced. Surprisingly, the model shows no substantial changes in the final temperature and salinity of the simulated outflow with reasonable variations in the drag coefficient, outflow rate or initial density anomaly. The volume transport does vary however. Table 3.2 summarizes the change in final outflow properties (denoted by the subscript $f$ ) found from changing the initial conditions.

\subsubsection{Changes in Initial Temperature and Salinity}

First, changes in the initial temperature and salinity are fairly straight forward to predict. Increasing the initial density by increasing the salinity or decreasing the temperature both act to increase the final density. Higher initial salinity leads to higher final salinity, etc. At first you might expect that this increase in density would reduce the bulk Froude number and stabilize the flow. We can rewrite the bulk Froude 


\begin{tabular}{|c||c|c||c|c|c|c|c|c|}
\hline & $\begin{array}{c}\rho_{\theta_{i}} \\
\mathrm{~kg} / \mathrm{m}^{3}\end{array}$ & $\begin{array}{c}Q_{i} \\
\mathrm{~Sv}\end{array}$ & $\begin{array}{c}S_{f} \\
\mathrm{pss}\end{array}$ & $\begin{array}{c}T_{f} \\
{ }^{\circ} \mathrm{C}\end{array}$ & $\begin{array}{c}\rho_{\theta_{f}} \\
\mathrm{~kg} / \mathrm{m}^{3}\end{array}$ & $\begin{array}{c}z_{f} \\
\mathrm{~m}\end{array}$ & $\begin{array}{c}h_{f} \\
\mathrm{~m}\end{array}$ & $\begin{array}{c}Q_{f} \\
\mathrm{~Sv}\end{array}$ \\
\hline \hline Basic Run & 28.50 & 0.75 & 36.31 & 12.01 & 27.62 & 948 & 221 & 2.2 \\
\hline$S_{i}=37.0$ & 27.92 & 0.75 & 36.21 & 12.26 & 27.50 & 836 & 225 & 1.7 \\
$S_{i}=38.5$ & 29.08 & 0.75 & 36.36 & 11.83 & 27.70 & 1030 & 220 & 2.8 \\
\hline$T_{i}=12.0^{\circ} \mathrm{C}$ & 28.74 & 0.75 & 36.24 & 11.56 & 27.65 & 983 & 222 & 2.5 \\
$T_{i}=14.0^{\circ} \mathrm{C}$ & 28.33 & 0.75 & 36.37 & 12.36 & 27.60 & 921 & 223 & 2.1 \\
\hline$h_{i}=75 \mathrm{~m}$ & 28.50 & 0.46 & 36.24 & 11.97 & 27.57 & 904 & 204 & 1.5 \\
$h_{i}=150 \mathrm{~m}$ & 28.50 & 0.92 & 36.34 & 12.03 & 27.64 & 966 & 229 & 2.6 \\
\hline$|\vec{u}|_{i}=0.5 \mathrm{~m} / \mathrm{s}$ & 28.50 & 0.62 & 36.28 & 11.98 & 27.60 & 931 & 214 & 1.9 \\
$|\vec{u}|_{i}=1.5 \mathrm{~m} / \mathrm{s}$ & 28.50 & 1.85 & 36.52 & 12.35 & 27.72 & 1033 & 246 & 4.3 \\
\hline$c_{d}=0.001$ & 28.50 & 0.75 & 36.16 & 12.04 & 27.50 & 788 & 169 & 2.9 \\
$c_{d}=0.005$ & 28.50 & 0.75 & 36.44 & 11.96 & 27.73 & 1126 & 239 & 1.9 \\
\hline
\end{tabular}

\begin{tabular}{|cc||c|c|c|c|c|c|}
\hline & & $\Delta S_{f}$ & $\Delta T_{f}$ & $\Delta \rho_{\theta_{f}}$ & $\Delta z_{f}$ & $\Delta h_{f}$ & $\Delta \frac{Q_{i}}{Q_{f}}$ \\
\hline \hline$\Delta S_{i}=1.5$ & $\left(\Delta \rho_{\theta_{i}}=1.16\right)$ & 0.15 & -0.43 & 0.20 & 194 & -5 & -0.18 \\
$\Delta T_{i}=-2.0$ & $\left(\Delta \rho_{\theta_{i}}=0.42\right)$ & -0.13 & -0.80 & 0.05 & 62 & -1 & -0.06 \\
\hline$\Delta h_{i}=-75.0$ & $\left(\Delta Q_{i}=-0.46\right)$ & -0.10 & -0.06 & -0.07 & -62 & -25 & -0.05 \\
$\Delta|\vec{u}|_{i}=-1.0$ & $\left(\Delta Q_{i}=-1.46\right)$ & -0.24 & -0.37 & -0.12 & -102 & -32 & -0.09 \\
$\Delta c_{d}=-0.004$ & & -0.28 & 0.08 & -0.23 & -338 & -70 & -0.14 \\
\hline
\end{tabular}

Table 3.2: Model Sensitivity to Initial Conditions. The initial conditions are evaluated with $S_{i}$ between 38.5 and $37.0 \mathrm{pss}, T_{i}$ between 12.0 and $14.0^{\circ} \mathrm{C}, h_{i}$ between 75 and $150 \mathrm{~m},|\vec{u}|_{i}$ between 0.5 and $1.5 \mathrm{~m} / \mathrm{s}$, and $c_{d}$ between 0.001 and 0.005 . All other parameters are held fixed with the values specified in table 3.1 while one parameter is varied. $Q_{i}$ and $Q_{f}$ are the initial and final layer transports respectively $\left(1 \mathrm{~Sv}=10^{6}\right.$ $\left.\mathrm{m}^{3} / \mathrm{s}\right)$.

number as a geostrophic Froude number (as in PB93),

$$
F r_{g}=\frac{U_{g}}{\left(g^{\prime} h\right)^{\frac{1}{2}}} \approx\left(\frac{g^{\prime}}{H}\right)^{\frac{1}{2}} \frac{\alpha}{f},
$$

where $g^{\prime}=g \delta \rho / \rho_{o}$ and $\alpha$ is the bottom slope. An increase in the initial density will increase the geostrophic velocity, $U_{g} \sim g^{\prime} \alpha / f$ and thus increase the Froude number, $F r_{g}$. The indirect effect of increasing the flow speed leads to more entrainment and thus the initial density anomaly is reduced. For instance, increasing the initial salinity 

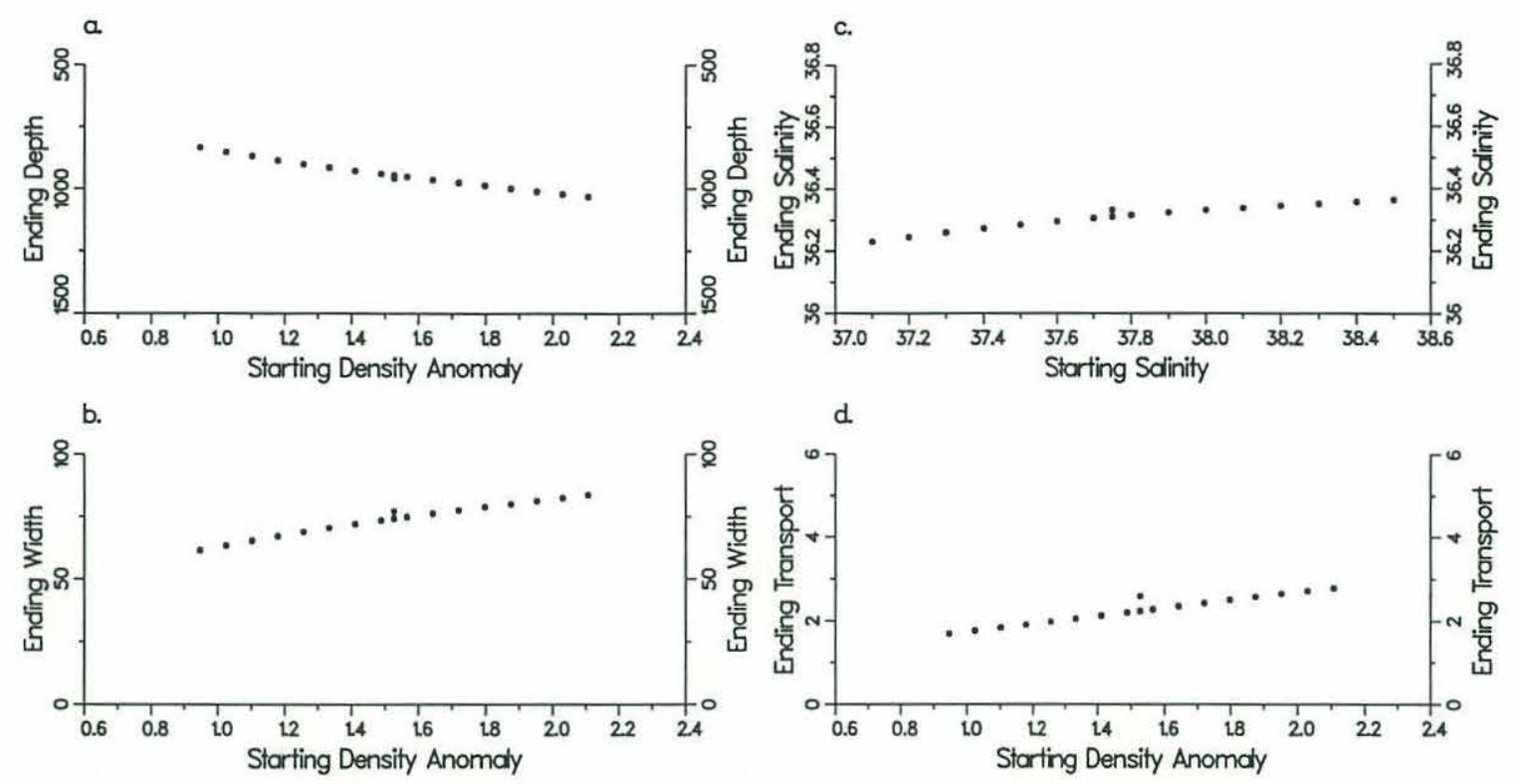

Figure 3.14: Final simulated outflow properties for different initial salinity. Salinity is varied from 37.0 to 38.5 pss which corresponds to an initial density anomaly range from 0.9 to $2.1 \mathrm{~kg} / \mathrm{m}^{3}$. a) Initial density anomaly versus the final equilibrium depth. b) Initial density anomaly versus final width of the simulated outflow. c) Initial salinity versus equilibrium salinity. d) Initial density anomaly versus ending transport.

from 37.0 to 38.5 leads to a density increase of $1.2 \mathrm{~kg} / \mathrm{m}^{3}$. However, the mixed water is only increased by $0.2 \mathrm{~kg} / \mathrm{m}^{3}$. Only $17 \%$ of the initial density anomaly survives the increased mixing. Thus, increasing the initial density anomaly induces a strong negative feedback by increasing the mixing, and as a consequence only sligthly denser mixed water can be produced by increasing the initial density.

\subsubsection{Changes in the Initial Height}

The initial height also has a very small effect on the final properties of the layer, but does significantly affect the predicted total volume transport. Decreasing the initial height from $150 \mathrm{~m}$ to $75 \mathrm{~m}$ leads to mixed water that is slightly colder and fresher, suggesting an increase in entrainment. We expect that decreasing the initial height will increase the Froude number (equation 3.40), initiating stronger mixing 
and leading to a colder, fresher and shallower mixed water. But in fact, the initially thin layer entrains only $1.0 \mathrm{~Sv}$ while the thicker layer entrains $1.7 \mathrm{~Sv}$ : the thicker layer actually entrains more water. The increased entrainment of the initially thicker outflow layer is, at first, counter intuitive.

Doubling the initial height of the outflow also doubles the initial outflow transport and thereby doubles the buoyancy flux. The thin layer has smaller initial buoyancy flux and is therefore modified very quickly by the increased initial entrainment (i.e. a higher initial Froude number as expected from equation 3.40). This reduces the buoyancy and, more importantly, the buoyancy forcing, $g^{\prime} \alpha$. A decrease in height also increases the effect of friction, which is inversely proportional to height, $c_{d} U^{2} / H$. These two effects together reduce the speed of the thin layer which cuts off the entrainment. The thicker layer maintains a higher Froude number for much longer because the buoyancy of the flow is not eroded as quickly. The net effect is more total entrainment in the thick layer. To produce fresher, cooler and shallower water however, the thicker layer needs to entrain at least twice as much in order to reduce its large initial buoyancy flux. So, although the final total outflow transport is greater for the initially thicker layer, the thick layer contains a lower percentage of ambient water (i.e. the thicker layer increases its initial transport 2.8 times (or $Q_{f} / Q_{i}=2.8$ ), versus 3.3 times for the initially thin layer).

\subsubsection{Changes in the Initial Speed}

The initial buoyancy flux is also important to consider in order to predict the effect of varying the initial speed. You might expect that increasing the speed would increase the Froude number and initiate more mixing (equation 3.40). This is confirmed by two different initial velocity solutions listed in table 3.2. The higher initial velocity solution entrains $2.5 \mathrm{~Sv}$ versus $1.3 \mathrm{~Sv}$ entrained by the lower velocity 

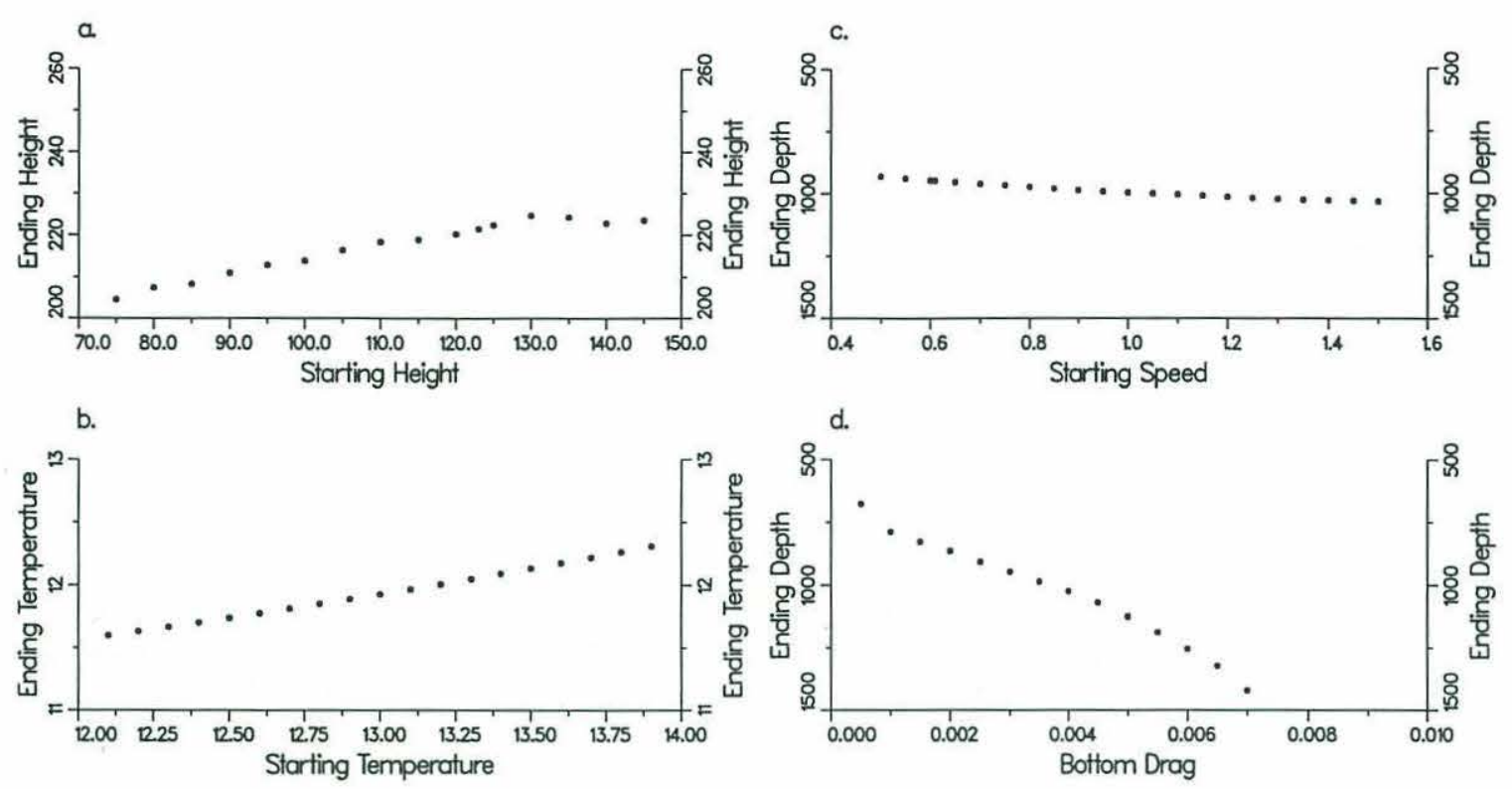

Figure 3.15: Model Sensitivity to varying initial height, temperature, speed and bottom drag coefficient. All parameters are held fixed while only one condition is varied. a) initial height versus final height. b) initial temperature versus equilibrium temperature. c) initial speed versus ending depth. d) bottom drag coefficient versus equilibrium depth.

solution. However, the higher velocity solution increases its transport only 2.3 times (i.e. $Q_{f} / Q_{i}=2.3$ ) while the lower velocity solution increases its transport 3.1 times. The temperature and salinity of the higher velocity solution are warmer and colder because the increased entrainment was not enough to modify the larger buoyancy flux. For the entrainment to affect the predicted mixed water of the simulated outflow, when the outflow velocity (and buoyancy flux) was tripled, the outflow would need to more than triple the amount of entrained water. In this case, the entrained water does not quite double as the initial velocity is tripled.

Why didn't it mix more? This increased mixing has a negative feedback on the system by decreasing the velocity through entrainment stress as well as bottom drag (see figure 3.2). Bottom and entrainment stress reduce the speed and Froude number, shutting off mixing. 


\subsubsection{Changes in the Bottom Drag Coefficient}

More dramatic variations occur to the simulated outflow as the bottom drag coefficient is varied (figure $3.15 \mathrm{~d}$ ). The final depth of the simulated outflow, for example, varies by an order of magnitude if $c_{d}$ varies from 0.001 to 0.01 . However, the change in final depth is relatively flat for bottom drag coefficients in the range of 0.001-0.005 and recall that Johnson et al. (1993) estimated $c_{d} \approx 0.0025$ for the Mediterranean outflow. Model solutions using two extreme $c_{d}$ coefficients are listed in table 3.2. The equilibrium properties of the simulated outflow are determined by two important effects of the bottom drag coefficient. For lower bottom drag, the flow speeds are less damped, thus raising the Froude number and initiating increased mixing. For higher bottom drag the flow is decelerated, decreasing the Froude number. Stronger bottom stress allows the flow to cross $f / h$ contours. Together, these two effects of increased $c_{d}$ allow the flow to descend further down the continental slope, maintaining a greater portion of its initial density contrast.

In the limit of very large drag coefficient, i.e. as $\mathrm{J} / \mathrm{f}$ becomes large, we can define a frictional Froude number by scaling the flow speed $U$ with the frictional 'uniform flow' solution discussed in section 3.2. The frictional Froude number is

$$
F r_{f}=\frac{u_{f}}{\left(g^{\prime} h\right)^{\frac{1}{2}}} \approx\left(\frac{g^{\prime} \alpha h}{c_{d}}\right)^{\frac{1}{2}} \frac{1}{\left(g^{\prime} h\right)^{\frac{1}{2}}} \approx\left(\frac{g^{\prime}}{c_{d}}\right)^{\frac{1}{2}}
$$

where we have used the fact that $u_{f} \sim g^{\prime} \alpha / J$ and $J \sim U c_{d} / h$. From this definition of a Froude number we can see more clearly that increasing the drag coefficient directly decreases the Froude number and thus suppresses mixing. For $\left(c_{d}, g^{\prime}\right) \ll 1$, $\mathrm{Fr}_{f}$ suggests that the mixing is more sensitive to changes in the drag coefficient than changes in $\mathrm{g}^{\prime}$. Figure $3.15 \mathrm{~d}$ confirms that for large $c_{d}$ the variations in final depth and density (not shown) become increasingly sensitive to the changes of the drag coefficient (i.e. the slope of the final depth versus $c_{d}$ curve begins to increase dramatically for $\left.c_{d}>0.006\right)$. For the Mediterranean simulation however we note 


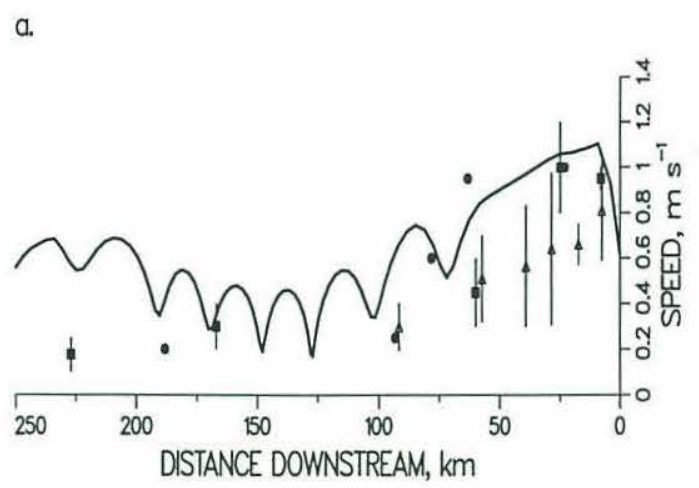

b.

ENTRANMENT RATE

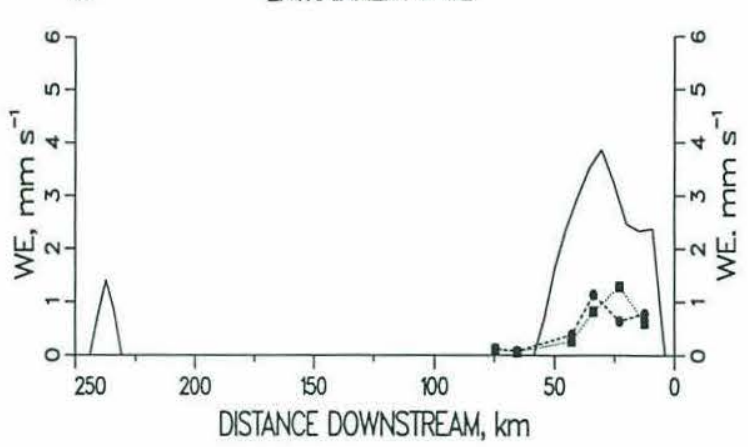

Figure 3.16: Model Solution for $c_{d}=0.001$. a) Current speed along the axis of the plume (compare with figure 3.7b). The 1988 Cadiz Expedition data is shown with error bars and open triangular symbols. Data with error bars and solid square symbols are from Smith (1975), data without error bars is from Heezen and Johnson (1969). b) Entrainment rate of the simulated outflow (solid line). Two different entrainment estimates discussed in chapter two are plotted: the entrainment from the salt equation is shown with square symbols and dotted line, the entrainment estimated from the increase in transport is shown with round symbols and dashed line (compare with figure 3.11 ).

that the flow is approximately geostrophic and $\mathrm{J} / \mathrm{f}$ is at most $3 / 10$ and this 'uniform flow' scaling does not apply. We will take this issue up further in the next section when we compare the relative sensitivity of the changes in buoyancy anomaly to drag coefficient in the low friction regime.

In the low friction regime changes in the final properties are much less drastic, however an unusual change in the location of the mixing occurs. Figure 3.16 shows the solution for a decreased $c_{d}=0.001$. The simulated outflow maintains its high velocity and the inertial oscillations discussed earlier are not as rapidly damped by bottom friction. The speed and path (not shown) of the simulated outflow executes a more pronounced cycloid motion (also apparent in the cusped velocity). The high velocity downstream induces a second, weaker mixing event $235 \mathrm{~km}$ downstream of the origin, near where the topographic slope increases (see figure 3.10c) and the flow accelerates. Thus the character of the solution changes slightly, by the introduction of a second mixing event. 
The second mixing event occurs as $c_{d}$ is decreased and does not appear by varying any of the other initial conditions. Changes in the ambient density profile can also produce two separate mixing regions, however. For instance, uniformly warming the profile below 600 dbar by $1.0^{\circ} \mathrm{C}$ (chosen so that the ambient profile was still statically stable) produces slightly less dense mixed water with $(\mathrm{T}, \mathrm{S})$ of $\left(11.92^{\circ} \mathrm{C}, 36.20\right)$ as compared to $\left(12.01^{\circ} \mathrm{C}, 36.31\right)$ for the standard model run, because of a second mixing region located $240 \mathrm{~km}$ downstream ${ }^{4}$. By decreasing the density of the ambient water, we have increased the density anomaly which forces strong topographic acceleration where the flow encounters the large slopes near Cape St. Vincent. The Froude number becomes large and mixing is reinitiated.

\subsubsection{Summary}

The model appears to be remarkably insensitive to changes in the initial conditions, but much more sensitive to the bottom drag coefficient, $c_{d}$, and the ambient density profile. Table 3.2 shows that the mixed water properties all lie near $27.62 \pm$ $0.02 \mathrm{~kg} / \mathrm{m}^{3}$ with $\mathrm{T}=12.03 \pm 0.07{ }^{\circ} \mathrm{C}$ and $\mathrm{S}=36.32 \pm 0.03$ pss. These mixed water types are all within the range expected for the outflow and we could not rule any of these solutions out. The initial conditions of the flow seem to have a secondary influence on the final properties of the simulated outflow than external conditions like bottom drag and ambient stratification, parameters the model cannot predict. The insensitivity of the model solution for the mixed water is due largely to a strong feedback effect of mixing.

\footnotetext{
${ }^{4}$ Other initial conditions can also lead to a second stage mixing with less dramatic warming of the ambient profile. For instance, the initial conditions $U_{0}=1.0 \mathrm{~m} / \mathrm{s}, T_{0}=13.4^{\circ} \mathrm{C}, S_{0}=37.9 \mathrm{can}$ lead to a less dense mixed water cooled from $12.27^{\circ} \mathrm{C}$ to $12.15^{\circ} \mathrm{C}$ and freshened from 36.5 to 36.45 by warming the ambient density profile only $0.2^{\circ} \mathrm{C}$.
} 
An essential aspect of this sensitivity study is to assess how sensitive the model is to the initial condition and parameters. When the friction is very large the bottom drag has a substantial effect but we note here that the Mediterranean outflow lies in the nearly geostrophic limit. In section 3.2 we found that the steady solution of a nonentraining particle on a slope subject to friction and the Coriolis acceleration has magnitude $\left|\vec{u}_{s}\right|=\frac{g^{\prime} \alpha}{\left(f^{2}+J^{2}\right)^{1 / 2}}$. We can therefore define a 'mixed' Froude number that includes the effects of friction on the speed and from which we can recover the geostrophic and frictional limits $F r_{g}$ and $F r_{f}$.

$$
F r_{\text {mixed }}=\frac{\left|\vec{u}_{s}\right|}{\left(g^{\prime} h\right)^{\frac{1}{2}}}=\left(\frac{g^{\prime}}{h}\right)^{\frac{1}{2}} \frac{\alpha}{\left(f^{2}+J^{2}\right)^{\frac{1}{2}}}
$$

For the Mediterranean outflow, $\mathrm{J} / \mathrm{f}$ is at most 0.3 , near the geostrophic limit. If we define $J=J_{o}+J^{\prime}$ and $g^{\prime}=g_{o}+\Delta g$ and assume $J / f$ is small then we can rewrite this as

$$
F r_{\text {mixed }}=\left(\frac{g_{o}+\Delta g}{h}\right)^{\frac{1}{2}} \frac{\alpha}{f}\left[1-\frac{J_{o}}{f}-\frac{J^{\prime}}{f}+\left(\frac{J_{o}+J^{\prime}}{f}\right)^{2}-\ldots\right]
$$

$\mathrm{Fr}_{\text {mixed }}$ depends linearly on changes in the friction but as the square root for changes in the $g^{\prime}$. For small $g^{\prime}, \mathrm{Fr}_{\text {mixed }}$ is more sensitive to changes in the density anomaly than friction in the nearly geostrophic regime. Further we know that $\mathrm{J} / \mathrm{f}$ is small but the changes in $g^{\prime}$ could be of order one. If $\Delta g / g^{\prime}$ is small then we can expand the square root of $g^{\prime}$ to find

$$
F r_{\text {mixed }} \approx\left(\frac{g_{o}}{h}\right)^{\frac{1}{2}} \frac{\alpha}{f}\left[1-\frac{J_{o}}{f}+\frac{\Delta g}{2 g_{o}}-\frac{J_{o}}{f} \frac{J^{\prime}}{J_{o}}+\text { HigherOrderTerms }\right]
$$

Then we see that we could allow large changes in $\mathrm{J}$ and still have $J<f$. In the Mediterranean where $J / f \sim 2 / 10$, this indicates that for a given percentage change in $g^{\prime}$ (i.e. a given $\Delta g / g^{\prime}$ ), the friction would need to change by 2.5 the same percentage to have the same effect on the Froude number. A careful look at table 3.2 confirms that doubling the initial buoyancy anomaly from $\sim 1$ to $\sim 2 \mathrm{~kg} / \mathrm{m}^{3}$ (by increasing the initial salinity from 37.0 to $38.5 \mathrm{pss}$ ) increases the final density by $0.2 \mathrm{~kg} / \mathrm{m}^{3}$. The bottom drag coefficient (and hence $J$ ) must be increased by a factor of 5 to produce 
the same mixed water density. Note that although we do not know the exact value of the bottom drag coefficient, it is very unlikely to range over such extremes. For a better estimate of the sensitivity of the model we need to consider the plausible or dynamic range of the variables. In this case, the density anomaly could vary between 1-2 $\mathrm{kg} \mathrm{m}^{-3}$ while the bottom drag will not vary between $1-5 \cdot 10^{-3}$. Therefore, the model is more sensitive to changes in the initial buoyancy anomaly than bottom drag coefficient. The ambient stratification thus plays a crucial role because the strong mixing modifies the outflow density but the ambient density profile is held fixed (which is reasonable for the time scales involved in the these overflows, typically 10 days for the Mediterranean outflow). The outflow properties thus tend towards the density of the ambient waters. 


\subsection{Conserving Potential Vorticity to Obtain a Constraint on the Width}

Several methods have been developed to estimate the width of the simulated outflow. The model as described in section 3.3 uses a width based on the assumed 'Ekman spreading' of the flow. In an earlier version of this model, the width was set $a$ priori as a specified linearly increasing function based on data from Smith (1975). As shown in figure 3.9, the Ekman number width function grows fairly linearly and there was little change in the solution when an explicitly linear function was used. In this section, we use a method for specifying the width which is at the opposite end of the spectrum from the frictional spreading currently used by assuming that the outflow conserves potential vorticity. Stommel and Arons (1972) first applied this idea to examine the broadening of deep western boundary currents. The basic assumption is that deep boundary currents are formed from uniform potential vorticity source waters that geostrophically adjust to form boundary currents on a slope. The approach is to invert the potential vorticity at any point downstream to obtain an estimate of the local width. We will closely follow the Stommel and Arons (1972) derivation but we will only need to apply three boundary conditions to solve for the width instead of the four conditions required for the full height solution and we will use the more realistic boundary condition for the Mediterranean outflow that requires the height to vanishes at the edges of the outflow. The derivation follows.

\subsubsection{Basic Equations}

The geometry of a cross-section of the flow is shown in figure 3.17. The bottom depth is given by $D(x)=-\alpha x$, where $\alpha$ is the bottom slope. Assuming that the flow conserves potential vorticity and that the potential vorticity within the layer is 


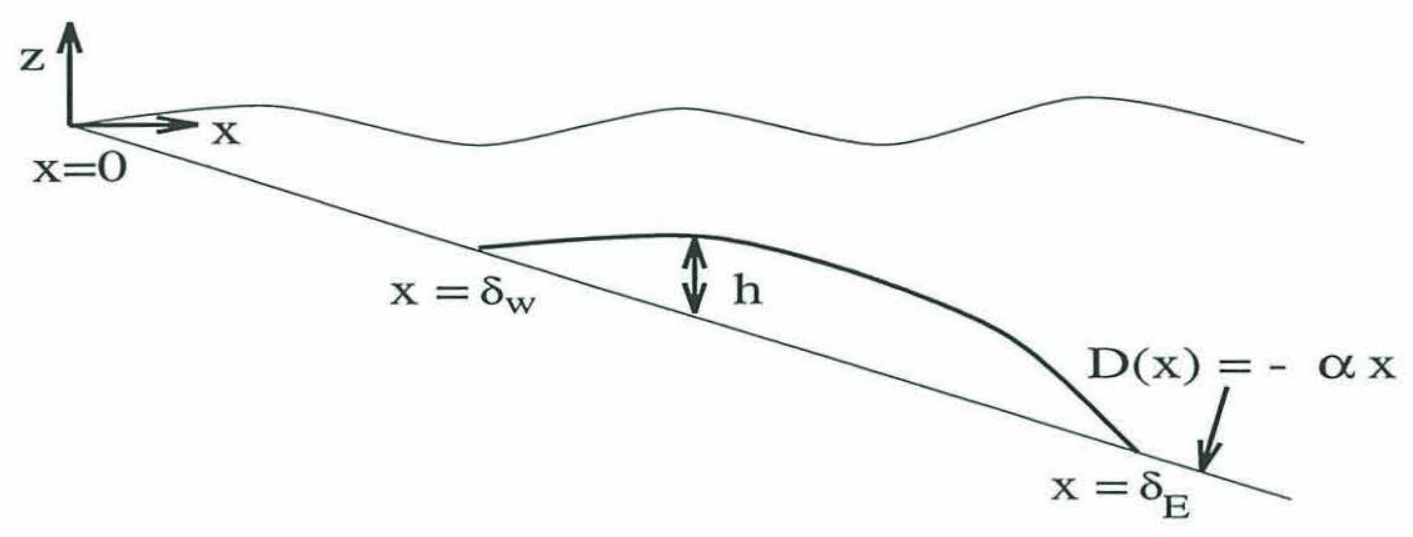

Figure 3.17: Schematic cross-section of layer of uniform density sitting on a slope. $D(x)$ is the bottom topography with slope $\alpha, h(x)$ is the layer thickness above the bottom.

uniform, then the motion can be described by

$$
\frac{f+\partial v / \partial x}{h(x)}=\frac{f}{H_{\circ}}
$$

and

$$
v=\frac{g^{\prime}}{f} \frac{\partial}{\partial x}(D(x)+h(x)),
$$

where $g^{\prime}$ is the reduced gravity $g \delta \rho / \rho_{o}$ and we have assumed that $\frac{\partial u}{\partial y} \ll \frac{\partial v}{\partial y}$. The potential vorticity at the source is given by $f / H_{o}$. Note that $H_{o}$ need not be the physical height, but can be thought of as the height to which the source water needs to be stretched so that the relative vorticity is negligible (i.e. an 'equivalent depth').

Combining equations 3.41 and 3.42 , yields a single equation for $\mathrm{h}$,

$$
\frac{\partial^{2} h}{\partial x^{2}}=\frac{f^{2}}{g^{\prime} H_{\circ}}\left(h-H_{\circ}\right) .
$$

To isolate the nondimensional parameters that describe the width, we can nondimensionalize this equation by assuming the following scales:

$$
\begin{aligned}
L_{r} & =\frac{\left(g^{\prime} H_{o}\right)^{\frac{1}{2}}}{f} \\
h & =H_{o} h^{\prime} \\
x & =L_{r} x^{\prime}
\end{aligned}
$$


Then equation 3.43 can be rewritten in non-dimensional form as

$$
\partial_{x^{\prime} x^{\prime}} h^{\prime}=h^{\prime}-1 .
$$

The solution to this equation is simply

$$
h^{\prime}=p e^{-x^{\prime}}+q e^{x^{\prime}}+1
$$

The width of the flow can be determined by requiring our solution to satisfy appropriate boundary conditions. In effect, we are inverting the potential vorticity equation 3.41 , subject to geostrophy (equation 3.42 ). The key to the implementation of this method will be the specification of the potential vorticity $f / H_{o}$.

\subsubsection{Possible Boundary Conditions}

To obtain a complete solution, we need to specify four boundary conditions to determine the coefficients $p, q$ and the edges of the flow $\delta_{w}^{\prime}, \delta_{e}^{\prime}$. We do not need the full height solution however, and instead require an equation for the width of the flow which is defined as $\Delta \delta=\delta_{e}^{\prime}-\delta_{w}^{\prime}$. Therefore, we require only three boundary conditions. ${ }^{5}$ Some choices include:

1. Specify $h$ at $\delta_{e}$
(a) assume $h\left(\delta_{e}\right)=h_{e}$, or
(b) assume $h\left(\delta_{e}\right)=0$.

2. Specify $h$ at $\delta_{w}$ :

(a) assume there is a wall at $\delta^{*}$, then $\delta_{w}=\delta^{*}$, or

(b) assume $h\left(\delta_{w}\right)=0$.

\footnotetext{
${ }^{5}$ Note that here we stray from Stommel and Arons (1972): they used sligthly different boundary conditions and solved for the full height solution.
} 
3. Assume the flow is next to a stagnant region:

(a) assume $v\left(\delta_{e}\right)=0$, and/or

(b) assume $v\left(\delta_{w}\right)=0$.

4. Specify the transport:

$$
Q=-\int_{\delta_{w}}^{\delta_{e}} v h d x
$$

\subsubsection{Application}

Initially, for simplicity, we assume that the outflow is bounded on both sides by stagnant regions and the total transport is given. Equation 3.42 can be nondimensionalized and rewritten as

$$
\left.v\right|_{\delta_{e}, \delta_{w}}=\left.\frac{g^{\prime}}{f} \frac{H_{o}}{L_{r}}\left(\frac{d h^{\prime}}{d x^{\prime}}-\alpha \frac{L_{r}}{H_{o}}\right)\right|_{\delta_{e}, \delta_{w}}=0
$$

Define $\sigma=\alpha \frac{\mathrm{E}_{\mathrm{r}}}{H_{\mathrm{o}}}$ which indicates the relative importance of the bottom slope in determining the velocity. Substituting in our solution 3.45 , this gives us

$$
\left.\left(-p e^{-x^{\prime}}+q e^{x^{\prime}}\right)\right|_{\delta_{e}, \delta_{w}}=\frac{\alpha L_{r}}{H_{o}}=\sigma .
$$

Applying equation 3.46 at both edges of the flow $x^{\prime}=\delta_{e}$ and $x^{\prime}=\delta_{w}$, we can solve for $p$ and $q$.

$$
p=\frac{-\sigma e^{\delta_{e}^{\prime}}}{1+e^{\delta_{e}^{\prime}-\delta_{w}^{\prime}}} \text { and } q=\frac{\sigma e^{-\delta_{w}^{\prime}}}{1+e^{\delta_{e}^{\prime}-\delta_{w}^{\prime}}}
$$

The transport boundary condition is more complicated. First we nondimensionalize the transport boundary condition 4 and find that the transport, $Q$, can be rewritten in dimensionless terms, $\tau$, by

$$
\tau=\frac{f}{g^{\prime} H_{o}^{2}} Q
$$

Substituting our solution 3.45 and the coefficients p and q, yields

$$
\Delta \delta=\frac{\tau}{\sigma}+2 \tanh \left(\frac{\Delta \delta}{2}\right)
$$



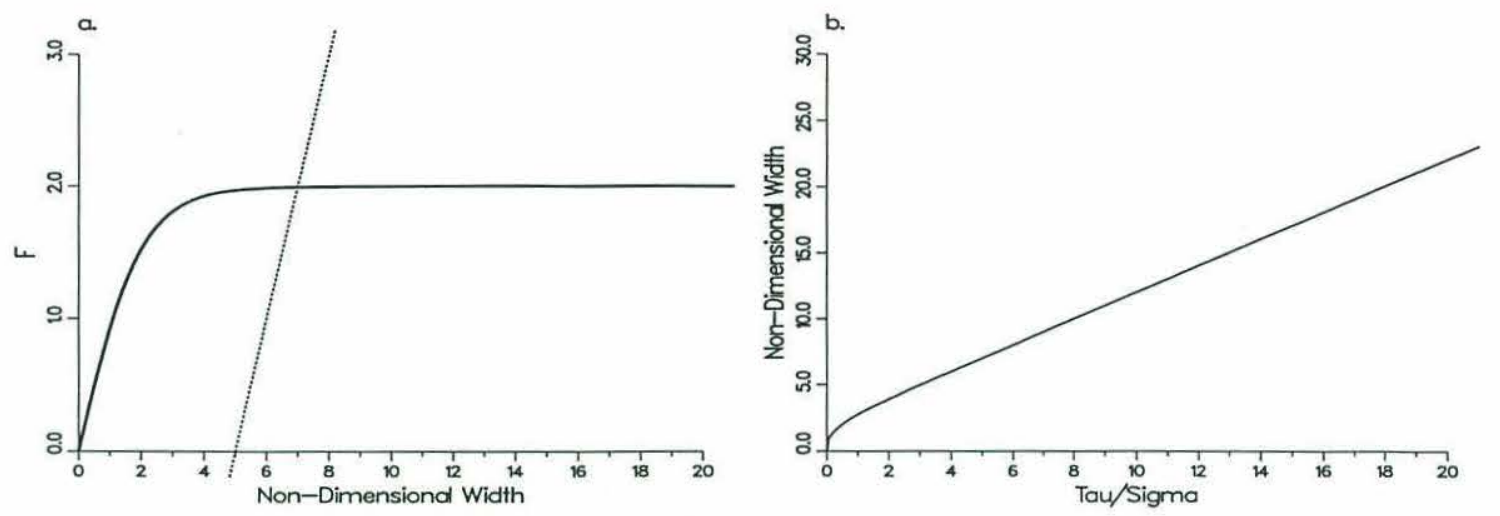

Figure 3.18: a) The solution to the transcendental equation 3.47 for the nondimensional width, $\Delta \delta$, is the intersection between the two functions: $f_{1}(\Delta \delta)=\Delta \delta-\tau / \sigma$ (dotted line), and $f_{2}(\Delta \delta)=2 \tanh (\Delta \delta / 2)$. Plotted here $\tau / \sigma=5$. We anticipate that the solution will grow linearly with $\tau / \sigma$. b) Non-dimensional width, $\Delta \delta$, evaluated from equation 3.47.

where $\Delta \delta=\delta_{e}^{\prime}-\delta_{w}^{\prime}$. This is a simple transcendental equation for the nondimensional width $\Delta \delta$ and it isolates the importance of the parameter $\tau / \sigma$. Everything is specified and we may solve this equation with Newtons method. Note that we have only used three boundary conditions to obtain this equation and we need not specify any other boundary conditions because we do not need or care about the complete height solution. The solution to this transcendental equation can be thought of as the intersection of the two curves

$$
\begin{aligned}
& f_{1}(\Delta \delta)=\Delta \delta-\tau / \sigma \\
& f_{2}(\Delta \delta)=2 \tanh (\Delta \delta / 2)
\end{aligned}
$$

Figure 3.18a shows an example of these functions with $\tau / \sigma=5$. Figure 3.18b shows the solution as a function of $\tau / \sigma$. In the limit of large $\tau / \sigma$ the solution in dimensional terms is

$$
\text { width } \approx L_{r} \Delta \delta \approx 2 L_{r}+(\tau / \sigma) L_{r}
$$

Physically, the $2 L_{r}$ piece of the width can be thought of as the adjustment region on either side of the outflow. The $\tau / \sigma$ piece assures that the total transport condition is satisfied. In dimensional terms $\tau / \sigma=\left(\frac{f}{g^{\prime} H_{o}^{2}} Q\right) /\left(\frac{\alpha L_{r}}{H_{o}}\right) L_{r}=f Q /\left(\alpha g^{\prime} H_{0}\right)$. This result is at least consistent, although perhaps not very profound. Conservation of mass tells 
us that the transport $\approx$ width $\cdot U \cdot H$. If the velocity is approximately $g^{\prime} \alpha / f$, then we have

$$
\text { width } \approx \frac{Q}{U H} \approx \frac{Q f}{g^{\prime} \alpha H} .
$$

This solution, then, is the 'low relative vorticity' solution and is closely tied to the upstream potential vorticity, or equivalent depth (i.e. $H$ is approximately $H_{\circ}$ ).

A more consistent boundary condition for a bottom trapped density current, can be found by specifying that the height of the fluid vanishes at the boundaries (as well as specification of the transport). Since the potential vorticity is uniform in the layer, the relative vorticity must increase to compensate for the vanishing layer thickness. The algebra of this 'high relative vorticity' solution is slightly more complicated, ${ }^{6}$ but following exactly the same procedure outlined above we find another transcendental equation that depends on both $\tau$ and $\sigma$ separately.

$$
\tau=\left(\sigma^{2}-1\right)\left[1-\frac{1}{2} \tanh ^{2}(\Delta \delta)-\frac{1}{\cosh (\Delta \delta)}\right]+\sigma\left[\frac{\sinh (\Delta \delta)}{\cosh ^{2}(\Delta \delta)}-2 \tanh (\Delta \delta)+\Delta \delta\right]
$$

Figure $3.19 \mathrm{a}$ shows the intersection between the two functions, $f_{1}$ representing the left hand side of equation 3.48 (i.e. $f_{1}=\tau$ ), and $f_{2}$ representing the right hand side of equation 3.48. Figure $3.19 \mathrm{~b}$ shows the nondimensional width, $\Delta \delta$, as a function of $\tau$ for various values of $\sigma$. Both of these figures show that the width depends strongly on the slope and aspect ratio of the flow, $\sigma$, but only weakly on the transport of the layer, $\tau$. This 'high relative vorticity' solution produces much narrower currents. The boundary condition requiring that the thickness of the layer vanishes on the boundaries of the outflow is also more consistent with the outflow data discussed in chapter 2 .

\footnotetext{
${ }^{6}$ Note that we require that $\sigma>1$ to assure that there will be no flow reversal
} 

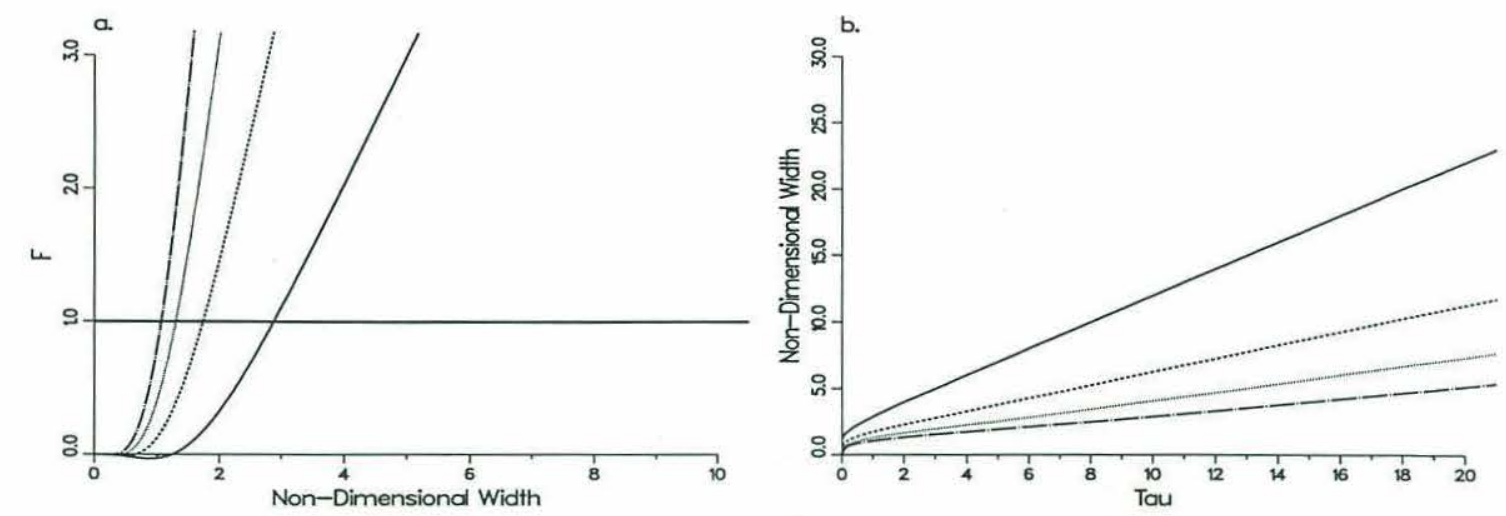

Figure 3.19: a) Graphically shows the solution to the transcendental equation 3.48 for the nondimensional width, $\Delta \delta$, for several values of $\sigma$. The solution is the intersection between the two functions: $f_{1}(\Delta \delta)=\tau$ (thick solid line), and $f_{2}(\Delta \delta)=$ right hand side of equation 3.48. Plotted here $\tau=1, f_{2}(\sigma=1)$ (solid line), $f_{2}(\sigma=2)$ (heavy dotted line), $f_{2}(\sigma=3)$ (dotted line), $f_{2}(\sigma=4)$ (chained line). b) Non-dimensional width, $\Delta \delta$, evaluated from equation 3.48 as a function of $\tau$ and $\sigma=1$ (solid line), $\sigma=2$ (heavy dotted line), $\sigma=3$ (dotted line), $\sigma=4$ (chained line).

\subsubsection{Implementation}

To implement this width determination in the simple plume model, we note that the isopycnals bounding the Mediterranean outflow intersect the bottom topography (see figure 2.5 and chapter 2). Therefore, we choose to apply the boundary condition with vanishing layer thickness at the edges. To prevent the inconsistent flow reversals possible for strong slopes, we limit the use of equation 3.48 for $\sigma>1$ and use equation 3.47 when $\sigma<1$.

One difficulty in inverting the potential vorticity to find the width of the flow, lies in the specification of the 'upstream' potential vorticity $f / H_{o}$. We use two approaches here. First, the potential vorticity would be set far upstream. We choose to specify the equivalent depth, $H_{o}$, as a constant and strictly conserve this value downstream. One possibility is to set $H_{0}$ to the initial height of the layer at the origin, $H_{o}=h(\vec{x}=0)$, for instance. A second method considered here, allows the 
potential vorticity to vary downstream as $f / h(\vec{x})$, using the local height predicted by the model.

Another difficulty in implementing this width specification is that this method implies that the flow has fully adjusted and has reached its asymptotic steady state. However, the Mediterranean outflow undergoes rapid adjustments near the strait. Initially, it is confined by a channel. Once it leaves the channel, it will adjust to some new width unconstrained by side walls. The 'Ekman spreading' width from the basic model run shown in figure 3.9a, increases rapidly once free of the channel near $25 \mathrm{~km}$. The 'potential vorticity' width (PVW, hereafter) however, specifies the fully adjusted state which is considerably wider than the initial channel width. Figure $3.9 \mathrm{a}$ shows the PVW calculated and carried passively in the model. Direct application of the PVW would more than double the width once the flow leaves the channel. This is certainly

physically unrealistic, since the edges of the flow which are assumed to be streamlines cannot move faster than internal gravity waves. Note that if the edges of the flow were not streamlines you might expect large horizontal hydraulic jumps as was found in the laboratory experiments of Pratt (1987). Also, this model implicitly assumes that the along-stream velocity, $\mathrm{U}$ is greater than the cross-stream velocity, V. Therefore, we arbitrarily limit the rate of change of width to be less than or equal to a constant, $C_{o}$, times the downstream velocity. We will see that the simulated outflow properties are fairly insensitive to the choice of $C_{o}$ or the 'upstream' potential vorticity.

\subsubsection{Results}

Figure 3.20 shows the simulated outflow speed, density anomaly, width and height obtained using the PVW with the 'upstream' potential vorticity determined from $f / h(\vec{x})$. The predicted model speed and density anomaly are virtually indis- 

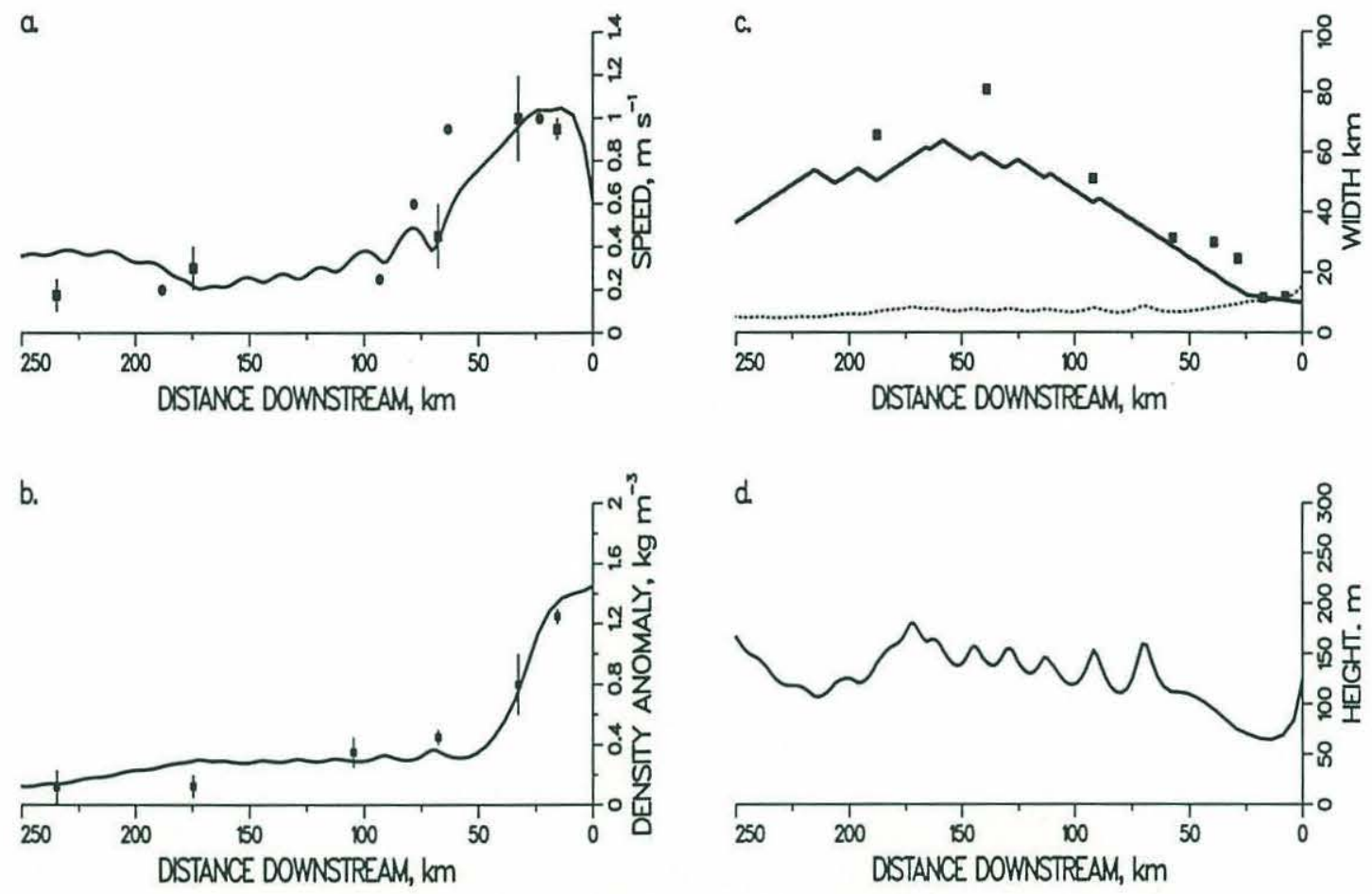

Figure 3.20: Model solution with variable potential vorticity and a maximum growth rate of the width of 0.5. a) Simulated outflow speed along the axis of the plume. The 1988 Cadiz Expedition data is shown with error bars and open triangular symbols (discussed in chapter two). Data with error bars and solid square symbols are from Smith (1975), data without error bars is from Heezen and Johnson (1969). b) Simulated outflow density anomaly along the axis of the plume. Density anomaly is the density difference between the plume and the surrounding waters. Plotted data from same source as in figure $3.20 \mathrm{a}$ c) Width of layer (solid line). GofCExp data width defined as the maximum extent of the 36.25 isohaline at each section (square symbols). The internal rossby radius is shown with the dashed line. The potential vorticity width is shown with the heavy solid line. d) Height of the simulated outflow layer. The 1988 Cadiz Expedition average height is shown with error bars representing \pm one standard deviation.

tinguishable from the basic model run (see figure $3.7 \mathrm{~b}$ and $3.9 \mathrm{a}, \mathrm{b}$ ). The width and height do show slight differences however.

To aid in our understanding of these results, we note that the PVW increases with $\tau / \sigma$ (figure $3.18 \mathrm{~b}$ ). This means that the nondimensional width, $\Delta \delta$ grows with increasing $\frac{Q f^{2}}{\alpha\left(g^{\prime} H_{0}\right)^{\frac{3}{2}}}$. The width initially increases and reaches a maximum of $63 \mathrm{~km}$ 


\begin{tabular}{|c||c|c||c|c|c|c|c|c|}
\hline & $\begin{array}{c}\rho_{\theta_{i}} \\
\mathrm{~kg} / \mathrm{m}^{3}\end{array}$ & $\begin{array}{c}Q_{i} \\
\mathrm{~Sv}\end{array}$ & $\begin{array}{c}S_{f} \\
\mathrm{pss}\end{array}$ & $\begin{array}{c}T_{f} \\
{ }^{\circ} \mathrm{C}\end{array}$ & $\begin{array}{c}\rho_{\theta_{f}} \\
\mathrm{~kg} / \mathrm{m}^{3}\end{array}$ & $\begin{array}{c}z_{f} \\
\mathrm{~m}\end{array}$ & $\begin{array}{c}h_{f} \\
\mathrm{~m}\end{array}$ & $\begin{array}{c}Q_{f} \\
\mathrm{~Sv}\end{array}$ \\
\hline \hline Basic Run & 28.50 & 0.75 & 36.31 & 12.01 & 27.62 & 948 & 221 & 2.2 \\
\hline \hline$C_{o}=1.00$ & 28.50 & 0.75 & 36.27 & 11.95 & 27.60 & 979 & 369 & 2.4 \\
$C_{o}=0.75$ & 28.50 & 0.75 & 36.30 & 11.99 & 27.62 & 996 & 356 & 2.3 \\
$C_{o}=0.50$ & 28.50 & 0.75 & 36.35 & 12.03 & 27.65 & 1021 & 346 & 2.2 \\
\hline \hline$H_{o}=125 \mathrm{~m}$ & 28.50 & 0.75 & 36.35 & 12.03 & 27.65 & 1008 & 366 & 2.2 \\
$H_{o}=1250 \mathrm{~m}$ & 28.50 & 0.75 & 36.35 & 12.03 & 27.65 & 1006 & 365 & 2.2 \\
\hline \hline$\Delta W / \Delta|\vec{x}|=0.5$ & 28.50 & 0.75 & 36.35 & 12.03 & 27.65 & 965 & 160 & 2.2 \\
\hline
\end{tabular}

Table 3.3: Model solution with different width specifications. Four different types of calculations are separated by double lines. First, the basic model run uses the 'Ekman spreading' equation 3.18 and is repeated here for convenience. Second, the variable upstream PVW is used with different maximum allowable growth rates. Third, a constant upstream PVW is used with different equivalent depths, $H_{o}$. Lastly, a constant linear increase in the width is used with a growth rate of 0.5. All other initial conditions used are listed in table $3.1 Q_{i}$ and $Q_{f}$ are the initial and final layer transports respectively $\left(1 \mathrm{~Sv}=10^{6} \mathrm{~m}^{3} / \mathrm{s}\right)$.

wide about $160 \mathrm{~km}$ downstream where the topographic slope is a minimum. Note that the PVW requires more broadening of the flow in this region: the 'Ekman' width is only $50 \mathrm{~km}$ wide. After $160 \mathrm{~km}$, the topographic slope begins to slowly increase and hence the PVW decreases. The 'Ekman' width begins to increase as the stronger slope increases the geostrophic flow speed and hence the Ekman number. The final widths of the two solutions are substantially different: the PVW is $23 \mathrm{~km}$, while the 'Ekman' width is $74 \mathrm{~km}$. The height of the flow is partially driven by the variations in the width through continuity. Therefore, the height mirrors the changes in the width: the PVW height is nearly the same as the 'Ekman' height for the first $80 \mathrm{~km}$, the PVW height is less between 80 and $180 \mathrm{~km}$ where the slope is small, and the PVW height is much larger as the PVW continues to narrow further downstream. Downstream of the initial arbitrary broadening, the PVW compares much more favorably with the observed width of the outflow than the Ekman spreading width. 


\section{Sensitivity}

Several experiments were conducted to examine the sensitivity of the results to variations in the potential vorticity specification and the maximum growth rate of the width. These are summarized in table 3.3. In general, the PVW mixed water is very similar in character to the basic model run using 'Ekman spreading' (i.e. the width grows like $2 E$ ). Increasing the maximum growth rate, initially allows the flow to spread much faster. Since the transport downstream increases with $w_{e} W$ (see equation 3.12), the initial increase in width allows slightly more entrainment into the plume. This in turn reduces the final density and decreases the final temperature and salinity because the flow entrains cold, fresh ambient waters. Specifying a constant source water potential vorticity, also produces very similar mixed water. Notice that decreasing the source potential vorticity from $f / 125 \mathrm{~m}$ to $f / 1250 \mathrm{~m}$ generates virtually no changes in the mixed water. Clearly, the final temperature and salinity properties are insensitive to the particular aspect ratio of the flow downstream of the entrainment region. For comparison, a model run that prescribes arbitrarily a linear, constant downstream growth rate (i.e. $2 E=0.5$ in equation 3.18 ) is also listed in table 3.3. Again, the downstream temperature and salinity are independent of the thin and broad aspect ratio of the layer far downstream: the final width exceeds 175 $\mathrm{km}$ and the height is only $160 \mathrm{~m}$.

\section{Comparison with 'Ekman Spreading'}

Changing the width parameterization does not vary the final temperature, salinity, density, or equilibrium depth significantly. The final solutions for various choices are virtually indistinguishable. However, the geometry and speed of the flow downstream are very different. The 'Ekman spreading' is a one way, irreversible process that produces a monotonically increasing width. The PVW, however, narrows as the 
topographic slope increases. This has an interesting effect on the velocity as well. Since the buoyancy forcing is calculated by taking the average slope over the width of the flow, decreasing the width changes the scale over which the slope is calculated. Near Cape St. Vincent actual slopes approach 0.06 and the maximum depth in the Gulf of Cadiz is near $2000 \mathrm{~m}$. Simple geometry will confirm that attempting to calculate the slope over widths greater than $25 \mathrm{~km}$ (given that the continental shelf is about $500 \mathrm{~m}$ deep), will underestimate the slope. Therefore the buoyancy forcing itself would be too low and the geostrophic flow speeds underestimated. For instance, the final flow speed of the 'Ekman spreading' simulated outflow run is only $0.14 \mathrm{~m} / \mathrm{s}$, compared to 0.28 for the PVW spreading.

Coupled with the decrease in final width is an increase in the final height from $221 \mathrm{~m}$ with 'Ekman spreading' to near $360 \mathrm{~m}$ with PVW. Figure 2.10 shows a vertical section of salinity approximately on the $8^{\circ} 30^{\prime} \mathrm{W}$ meridian which was taken during the 1988 GofCExp. If we define the 36.4 isohaline as marking the edges of the plume we see that the boundary current is anywhere from 20-30 km wide and between 250-500 $\mathrm{m}$ in height (even if we choose the 36.3 isohaline the width would only be $45 \mathrm{~km}$ ). Clearly, the PVW has the right order of magnitude for both the width and the height of the flow in this region, although the simple plume model and PVW assumed that the current was in contact with the bottom and the plume near Cape St. Vincent is not.

\section{Summary}

In conclusion, the PVW has the advantage that the model predicts that the flow will narrow and thicken downstream where the topography becomes steep. The PVW also predicts broader flows, as observed, where the slope is very small. The 'Ekman spreading' has the advantage that there is no adhoc maximum growth rate limit when the flow first exits the channel (although the assumption that the lowest edge moves 
twice as far downslope as the center of mass while the upper edge of the flow remains stationary has the flavor of tuning to it). For this simple model, it is interesting to note that two different methods at the inviscid and frictionally dominated ends of the spectrum, give essentially the same mixed water properties and transport. This is probably because the entrainment dominates the final bulk properties in this simple model. Perhaps a full potential vorticity evolution equation would be more realistic, including frictional effects. Undoubtably, Ekman spreading is important where the stresses on the flow are the largest (see also section 2.4). Downstream, where Stommel envisioned a frictionless geostrophic flow, potential vorticity conservation may define the adjustment (i.e. is closer to the observations). However, given that substantial changes in the initial potential vorticity had little effect on the flow properties, this might add unnecessary complications which would shroud the simplicity of the basic model.

\subsection{Summary and Conclusions}

In this chapter, we have used a simple model of a density driven plume to examine the dynamics of the Mediterranean outflow. Data analyzed from the GofCExp have been compared with the model solutions. Both data and model predict that the Mediterranean outflow, which has a temperature and salinity of $13.2^{\circ} \mathrm{C}$ and 37.75 pss near $6^{\circ} 20^{\prime} \mathrm{W}$, freshens and cools rapidly as the outflow entrains North Atlantic Central Water. The model predicts temperature, salinity, height, width, and depth of the plume remarkably similar to the observed properties of the data (see figures 3.8, 3.9). The model tends to overpredict the entrainment and transport of the plume (see figure 3.11 and 3.10c). Although we do not have a good estimate of the final velocity weighted temperature and salinity of the data near Cape St. Vincent, examination of figure 2.10 and the corresponding temperature data from the GofCExp, 
suggests that the final simulated outflow ranges in salinity from $36.45 \pm 0.15$ pss and in temperature from $12.15 \pm 0.65^{\circ} \mathrm{C}$. The simulated outflow ends with temperature and salinity of $12.01^{\circ} \mathrm{C}$ and 36.31 pss which is towards the colder fresher end of the range expected from the data, which is consistent with a slight overestimation of the entrainment. However, it should be noted that Ochoa and Bray (1991) found a total nonrecirculating transport of mixed Mediterranean water of $2.2 \mathrm{~Sv}$ near $8^{\circ} \mathrm{W}$, exactly the predicted simulated outflow transport.

Some other correlations between the simulated outflow and the data are summarized below.

- The simulated outflow accelerates to speeds in excess of $1 \mathrm{~m} / \mathrm{s}$ within the first $25 \mathrm{~km}$. The horizontally and vertically average speed calculated from the data decelerates, but the 'core' of the flow where the maximum salinity is found, accelerates to a maximum speed greater than $1.4 \mathrm{~m} / \mathrm{s}$. The Cadiz data also shows that the kinetic energy of the flow initially increases to section $\mathrm{C}$ (see section 2.6.3), even though the mean speed decreases (hence the larger error bars at section $\mathrm{C}$, near $30 \mathrm{~km}$ in figure $3.7 \mathrm{~b}$ ).

- The model overestimates the entrainment, speed and transport, while underestimating the initial height of the outflow. The overestimate of the speed of the simulated outflow leads directly to the underestimate of the height through continuity (as the layer accelerates it must thin to conserve mass). The overestimate of the speed also leads directly to an overestimate of the entrainment (and therefore the total transport): $w_{e}=w_{e}\left(R_{b},|\vec{u}|\right)$ (with $R_{b}$ matching the data fairly well). The speed seems to be overestimated due to the high buoyancy anomaly: even though the salinity, temperature and density (figure 3.8) match the data well, the density anomaly is too high (figure 3.7b). The ambient stratification is critical in determining the mixed water properties. Perhaps a 
model with an active upper layer would provide the variable ambient stratification that we expect.

- The model also predicts a nearly inertial turn similar to the inertial turn described in chapter 2 for the GofCExp data. The Rossby number during this broad turn occurring during the first half an inertial period reaches a maximum of 0.6 , while further downstream the cross-stream Rossby number is less than 0.1. The GofCExp data also indicated a cross-stream Rossby number of $\frac{1}{2}$.

- Large bottom and entrainment stress retards the model speed. Both bottom stress and entrainment stress reach a maximum in excess of $3 \mathrm{~Pa}$ close to 25 $\mathrm{km}$ downstream. In chapter 2 , we found that the maximum entrainment stress was only $0.8 \mathrm{~Pa}$, which is considerably lower than the entrainment stress in the model. This is due primarily to the low entrainment rate found in the data which was $0.0013 \mathrm{~m} / \mathrm{s}$ versus a maximum of $0.004 \mathrm{~m} / \mathrm{s}$ in the simulated outflow.

- Entrainment is highly localized along the path of the flow. Both the simulated outflow and the data suggest that the bulk of the entrainment occurs in less than $40 \mathrm{~km}$ of the path of the flow (about 15\% of the total path through the Gulf of Cadiz). The intermittent nature of the entrainment appears to be appropriately modeled by the Froude number formulation of Turner (1986). The data also suggest that the maximum entrainment occurs near the largest bulk Froude numbers of the flow.

- Uniform potential vorticity conservation can be used as a constraint on the width of the plume. The width based on this method matches some of the observed properties of the flow better than the PB93 technique which assumes that spreading is proportional to the Ekman number. However, this method necessarily ignores the time dependent adjustment required as the flow initially leaves the Strait of Gibraltar. 


\section{Chapter 4}

\section{Summary and Discussion}

Historical observations show that the outflow of Mediterranean water from the Strait of Gibraltar forms a distinct plume that flows along the northern continental slope in the Gulf of Cadiz and eventually separates into the North Atlantic as an intermediate water mass. As the flow moves through the Gulf of Cadiz the salinity decreases from 38.4 pss at the Strait of Gibraltar, to $36.45 \pm 0.15$ off the coast of Portugal. This entrainment of North Atlantic Central Water also reduces the temperature from about 13.1 to $12.15 \pm 0.65{ }^{\circ} \mathrm{C}$ before the outflow enters the open ocean.

This thesis examines the mixing and dynamics of the Mediterranean outflow and in particular, we addressed the location and intensity of the entrainment and the force balances that control the outflow's descent. To do this, observations of temperature, salinity and velocity collected during the Gulf of Cadiz Expedition in 1988 were analyzed and compared with several simple analytical and numerical models. 


\subsection{What does the data tell us?}

\section{Entrainment}

Entrainment is highly localized along the path of the flow, the bulk occurs in less than $15 \%$ of the total path of the flow through the Gulf of Cadiz. The outflow doubles its initial transport of $0.7 \mathrm{~Sv}$ to $1.5 \mathrm{~Sv}$ in the $60 \mathrm{~km}$ from section $\mathrm{B}$ to section $\mathrm{F}$ and the velocity weighted salinity decreases from 37.75 pss to 36.5 pss (chapter 2.5). Further downsteam, we find that the outflow transport reaches $1.9 \mathrm{~Sv}$ and the flow begins to separate from the bottom between 1100 and $1300 \mathrm{~m}$ depth (our section $\mathrm{H}$ ). These properties are consistent with historical observations although estimates of the total transport leaving the Gulf of Cadiz have been higher (between 2.2-3.0 Sv). The most significant result is that the entrainment is very localized along the path of the flow, the bulk occurring in the $30 \mathrm{~km}$ between section $\mathrm{B}$ and $\mathrm{D}$.

Mass and salt conservation statements can be used to explicitly estimate the entrainment into the plume. The inferred entrainment rate reaches a maximum at section $\mathrm{C}$ where most of the entrainment occurs. The maximum entrainment rate exceeds $30 \mathrm{~km} /$ year which is three orders of magnitude larger than typical vertical velocities like Ekman pumping or subduction in the open North Atlantic (chapter 2.6.4). The total entrainment is small relative to subducted waters in the North Atlantic however, local, extremely intense mixing occurs in the Mediterranean outflow.

\section{Richardson numbers}

Instability theory and laboratory experiments suggest that entrainment can be parameterized with a Richardson number (or equivalently a Froude number, Price 1979). We found that the Mediterranean outflow had consistently low gradient Richardson numbers at the interface with the overlying NACW, suggesting that the flow is po- 
tentially unstable to Kelvin Helmholtz instability. More importantly, we also found that there is a spatial correlation between areas of intense entrainment and low bulk Richardson numbers or Froude numbers (chapter 2.5, figure 2.23). This suggests that it might be possible to model this overflow using a Froude number based entrainment law such as Turner (1986).

\section{Dynamics of the current and descent}

The large entrainment rate suggests an interfacial stress that reaches a maximum of $0.8 \mathrm{~Pa}$ (chapter 3.8). The dominant stress that balances the buoyancy forcing is the bottom stress which reaches values above $5 \mathrm{~Pa}$ (chapter 2.6.1). It is this large bottom stress that allows the flow to cross isobaths and descend into the North Atlantic. Large, relatively slow currents like this one feel the earths rotation and accelerate only until deflected by the Coriolis force to follow $f / h$ contours (chapter 2.6.2). We used a simple geostrophic adjustment model to show that a density current with these scales and buoyancy would accelerate and rapidly align itself along a very shallow isobath in the absence of friction. Thus, friction is essential for the plume to descend at all.

\section{Spreading and horizontal structure}

The flow also seems to spread with the help of strong frictional forces. We do observe consistent spiraling of the velocity profile with height in the same sense as a classical Ekman spiral (at least above the velocity maximum) and the average velocity above and below the 'nose' or maximum velocity of the plume indicate an average veering of $8^{\circ}$. This angle is too small to account for all of the spreading observed, however. Vertically averaged (i.e. barotropic) velocities indicate large spreading angles, as large as $50^{\circ}$, with each edge of the flow moving apart. 


\section{Circulation above the outflow}

The Gulf of Cadiz must supply two sinks of mass to 1) feed the intense mixing into the plume and to 2) supply the Mediterranean Sea with inflow. We found that both of these sinks came from mid-depth below the upper 50 meters (chapter 2.5). The entrainment into the plume comes from the water in contact with it, in a density range between $\sigma_{\theta}=27.1-27.3$ (figure 2.19), which disappears closer to the strait after the flow is entrained. The inflow transport is relatively uniform above the plume and is concentrated in the shallower water towards the north (i.e. Spain), and falls generally in the range of $\sigma_{\theta}=26.7-27.1 \mathrm{~kg} / \mathrm{m}^{3}$. The surface waters (above 50 m) are dominated by a surface intensified jet moving towards the west and present predominantly offshore, in deeper water in all our XCP and geostrophic velocity sections (figure 2.8 and figure 2.9). We have not ascertained a source for this water east of our section A, nor are we aware of any other data corroborating its existence.

\subsection{What does the model tell us?}

A density driven model with Froude number dependent mixing and a quadratic bottom stress law was used to simulate the Mediterranean outflow (chapter 3). We showed that the properties and dynamics of the model compared well with some features of the observations described in chapter two. Specifically, the model predicts temperature, salinity and depth values very similar to the observations; the simulated

plume cools from 13.2 C at the western end of the strait to $12.0 \mathrm{C}$ at Cape St. Vincent, and the salinity freshens from 37.75 pss to 36.3 pss. Like the observed plume, the model entrains in a very localized area (less than $30 \mathrm{~km}$ ), increasing the transport to $2.2 \mathrm{~Sv}$. 
The physical parameters in the model that influence the plume's descent are the density anomaly, bottom slope, bottom friction, and the ambient stratification. Strong buoyancy forcing accelerates the plume down the bottom slope, increasing the Froude number. As the Froude number rises above one, mixing with the overlying North Atlantic water is initiated. The strongest mixing occurs close to the straits where the velocity and density anomaly are the greatest. This supports our hypothesis that the mixing can be parameterized through a bulk Froude (or Richardson) number. The model demonstrates the importance of inertia on the flow, showing strong oscillations as the flow executes a classic cycloid trajectory; the Rossby number is as high as 0.6 initially as the flow turns to align itself parallel to the local topography. Also like the data, the model predicts a frictional stress as high as 3 $\mathrm{Pa}$ retarding the flow initially. Farther downstream, the plume becomes a slightly damped geostrophic current with stresses less than $\frac{1}{2} \mathrm{~Pa}$ and a Rossby number less than 0.1 .

The model slightly overpredicts the outflow velocity and hence, the entrainment. We believe that a likely cause is the unrealistic assumption that the upper ocean is passive. The observations show (figure 2.4b) that the upper layer is far from passive and that more water enters the Mediterranean sea than leaves it. This upper level flow must be supported by a pressure gradient that would presumably act to slow the plume. Also, the rough topography in the Gulf of Cadiz is a likely source for internal waves, taking their energy from the mean flow or tides (Polzin, 1992). Geostrophic adjustment and entrainment would also radiate energy away into a stratified active ocean and would act as a sink on the plume momentum which is not represented in the model. 


\subsection{Discussions and Speculations}

\section{On spreading}

Using dissipation data, Rolf Lueck (1990) found friction velocities, $U_{*}$, on average between $0.01-0.02 \mathrm{~m} / \mathrm{s}$ confirming the high stresses retarding the flow. We can get a quick scaling of the turbulent bottom boundary layer by using Wimbush and Munk's (1970) estimate that the turbulent kinetic energy will extend away from the boundary approximately $h_{\delta}=\frac{0.4 U_{*}}{f}$, or in our case approximately 80 meters. This is an extremely large distance compared to most bottom boundary layers (more typically $10 \mathrm{~m}$, see Jones, 1989). Given also the steady nature of the flow we might expect to observe some sort of Ekman type balance throughout the entire plume, however, the spreading rate of the baroclinic flow is too small to match the observations. This suggests that some barotropic process is the dominant process governing the spreading of the flow. One contender for this barotropic process is the steering of the flow by small scale topographic features and hence unresolved pressure forcing. A related contender is a geostrophic adjustment process. For instance, the average topographic slope decreases downstream of section $\mathrm{D}$ where the flow begins to spread. The flow could diverge as each edge tries to follow its local isobath. A similar theory was advanced by Warren (1991) to explain the bifurcation of the Gulf Stream downstream of the Grand Banks (see also Stommel and Arons, 1972). This is essentially equivalent to the potential vorticity conservation argument we successfully used to describe the broadening in the simple model in chapter 3.5. For nearly geostrophic flow where $\mathrm{J} / \mathrm{f}$ is small (section 3.2), potential vorticity can be conserved and a fluid column will try to follow $f / h$ contour. As $\mathrm{J} / \mathrm{f}$ increases, friction and entrainment will break this lowest order process. The 'two cores' can then be thought of as the preferential volume flux along the two outer trajectories of the flow which become increasingly differentiated as the mix with NACW from different depths. 
The simple numerical model showed us that variations in the initial conditions reproduce a continuum of mixed waters with no preferential separation into different water classes. Observations have shown us that the outflow near the strait is highly stratified with only a small horizontal separation in core properties (see figures A.3 and 2.13). This suggests that the initial conditions at the Strait are unlikely to produce two distinct cores of 'product water', but should produce a continuum (unless there are time dependent pulses of water leaving the Strait, which has not yet been observed). The two 'cores' of outflow must be predominantly produced through a different mechanism, such as topographic steering combined with differential mixing.

It is interesting to note that the simple PB93 plume model showed a two step mixing when either the ambient profile was warmed, or the drag coefficient was lowered. The observations of the upper core also indicated a dominant mixing event near section $\mathrm{C}$ and another transport increase before section $\mathrm{F}$ where the slope by the upper core abruptly steepens (chapter 2.5). Both model and data suggest little change in the temperature and salinity of the flow during the second weak event. This is mostly because the high density anomaly has already been substantially eroded, and the entrained water has higher salinity and temperature (see figures 2.12). The point to note is that 1) in the observations, the upper core lies just below warm, fresh water that it will entrain $\left(13{ }^{\circ} \mathrm{C}\right.$ versus $11{ }^{\circ} \mathrm{C}$ above the lower core, figure 2.12) and 2) we can conceptually formulate a model of a two layer plume in which the lowest layer feels the bulk of the friction, while the upper layer feels less (i.e. the turbulence decays away from the bottom boundary), and therefore has a lower $c_{d}$ (perhaps more appropriately modeled as interfacial friction). Therefore, if the layer does spread baroclinically, the upper core might experience less friction and will mix with warmer ambient water. Like the numerical model, the conceptual model then favors a secondary mixing event and as well as warmer, fresher mixed water with density $\sigma_{\theta}=27.5$, as expected of the upper core. 


\section{On the influence on the general circulation}

One of the main purposes of this study was to understand the dynamics of one overflow in that hope that we would understand all overflows better (Price and Baringer, 1993). The long term goal is that we may predict the evolution of overflows and their influence on the climate of the past as well as the climate of the future. For instance, could the Mediterranean outflow become the dominant source of deep water in the North Atlantic or produce the warm, saline deep circulation of 50 million years ago (Brass et al., 1988)? The plume model described here gives us a simple even if incomplete way to begin to address some of these questions. This model can characterize how initial conditions at the sill affect the temperature, salinity and transport of the overflow waters where they merge with the open ocean by assuming the overflow dynamics are isolated from the general circulation.

Bryden and Kinder (1992), for example, ask how the properties at the Strait of Gibraltar would change given only a change in the geometry of the Strait, induced by sea level change for example. In particular, if the sill has only half its present nominal depth of $284 \mathrm{~m}$, they find that the outflow salinity would increase to $40.5 \mathrm{pss}$ (assuming the average evaporation over the Mediterranean was $66 \mathrm{~cm} / \mathrm{yr}$ ). This also implies a significantly reduced outflow transport of $0.44 \mathrm{~Sv}$ and an inflow transport of $0.5 \mathrm{~Sv}$. Bryden and Kinder ask what would the equilibrium depth be assuming these initial conditions. We can attempt to answer this question by using the simple plume model and assuming that the 'climate' of the North Atlantic maintains a similar ambient density profile and that the salinity difference where we initialize the model increases by a similar amount (i.e. increased by $2.1 \mathrm{pss}$ ). Initializing the model with these conditions at the Strait (i.e. $U_{o}=0.59 \mathrm{~m} / \mathrm{s}, H_{o}=74 \mathrm{~m}, Q_{o}=0.44 \mathrm{~Sv}$, $S_{o}=39.85 \mathrm{pss}$ ), we find that 'equilibrium' water entering the North Atlantic would be very slightly deeper and colder $\left(S_{f}=36.33 \mathrm{pss}, T_{f}=11.60^{\circ} \mathrm{C}, z_{f}=1054 \mathrm{~m}\right.$, $Q_{f}=2.5 \mathrm{~Sv}$ ) than the present overflow. 
The simple plume model shows that the ambient stratification is at least as important as the initial conditions in the marginal sea at setting the final properties of the Mediterranean water mass found in the open ocean. In fact, if the ambient stratification were significantly denser than the present day stratification, for instance with the characteristics found in the mid-ocean thermocline in the Iberian Basin where the Denmark Strait overflow water descends the continental slope in a similar density driven plume, then the Mediterranean outflow would become much colder and fresher and descend to $2000 \mathrm{~m}$, the bottom of the Gulf of Cadiz. This suggests that there are situations in which Mediterranean water may reach greater depths in the North Atlantic.

Given the success the simple model has in predicting the dynamics and flow properties as observed in the data, we are tempted to go further and ask the hypothetical question: what is the limit of any one parameter in the model that would allow the Mediterranean to descend to the bottom of the Gulf of Cadiz? We see that the ambient stratification can induce more mixing into the plume (two stage mixing appears when the ambient profile decreases its density), and less mixing as shown above. So clearly the long term climate of the open ocean is an essential part of the answer to this question and something that this model cannot predict. We can however speculate about what one initial condition could allow the plume to reach the bottom given the present ambient stratification. The answer is surprisingly that the only initial condition that could produce bottom water is a salinity in excess of 79 pss $\left(\sigma_{\theta}=61.53\right) .{ }^{1}$ Such high salinity water would require an extremely high evaporation rate over the Mediterranean and the changing dynamics of the exchange through the strait would undoubtably lead to different net exchange etc., thus changing the other

\footnotetext{
${ }^{1}$ Bottom water could also be produced with a sufficiently large bottom drag coefficient (larger than 0.008). However, we are loath to suggest that the bottom drag coefficient is a tunable parameter. We recognize that like the surface drag coefficient for predicting wind stress, the bottom drag coefficient is in principle an empirically observable quantity that will depend on many things including bottom roughness elements and suspended sediment to name a few.
} 
initial conditions of this model. Thus, we must consider the changing conditions in the upstream basin and the hydraulic control process at the strait to fully assess this question.

As we have seen in chapter 3 , the most important initial condition is the total buoyancy anomaly flux. We have seen that increasing the buoyancy and velocity tends to increase the penetration of the plume with depth, but that there is also a strong feedback which increases the entrainment. Although increasing the velocity cannot on its own create bottom water, perhaps a combimation of increased volume transport and buoyancy anomaly would reduce the amount of buoyancy flux required for the plume to reach the bottom of the North Atlantic. In any case, we must move towards coupling the upstream conditons and the hydraulic control at the strait to the general circulation which sets the ambient density.

To fully understand the effect of the Mediterranean overflow on climate, we need to link the initial conditions at the Strait to not only hydraulic control theories and the upstream air-sea fluxes in the Mediterranean Sea, but to the general circulation in the open ocean and we must include the necessary feedback on all of these processes. A first attempt at diagnostically linking overflows with the interior stratification has been done (Speer and Tziperman, 1990). Rhines and MacCready (1989) have advanced the theory that intense boundary mixing will control the interior circulation through a linear vorticity balance and the source/sink into the boundary. Through a greater understanding of the physics of overflow waters and how mixing produces different water masses, eventually a methodology can be developed to incorporate the end result of such mixing into general circulation models (GCM). Then questions of how the Mediterranean outflow influences deep water formation, how the open ocean and marginal sea processes feed back on each other, and how climatic scale variations in deep water characteristics change the global circulation might then be addressed. 


\section{Appendix A}

\section{Determining the Absolute}

\section{Velocity}

Historically, the flow is assumed motionless at the salinity minimum. Here we first set the along-stream flow to be motionless at the local temperature minimum above the salinity anomaly. What is the difference between the salinity minimum and the temperature minimum? The salinity minimum is the depth with the lowest salinity. The temperature minimum is nearly the absolute temperature minimum through most of the Gulf of Cadiz. It is the depth of minimum temperature and salinity above the warm salty Mediterranean water. The distinction between the temperature minimum and the temperature minimum is necessary because where the outflow begins to lift off the bottom the minimum temperature is below the outflow. Similarly, in the Strait of Gibraltar, although the outflow is warm and most stations exhibit a slight temperature minimum above the outflow, this temperature minimum disappears closer to the Mediterranean and the lowest temperature is at the bottom within the salty outflow, which is certainly not motionless. 


\section{A.1 Determining the top of the Mediterranean Outflow}

Station 97 in section E (figure A.1) has a typical profile which can be used to illustrate the difference between the salinity minimum and the temperature minimum. Figure A.1 shows the vertical profile of salinity and temperature for CTD station 97 with Mediterranean water present and CTD 98 with no Mediterranean water present. Note that these stations are within $6 \mathrm{~km}$ of each other and one contains Mediterranean outflow and the other does not. Clearly, the outflow has well defined horizontal limits. Station 98 represents the 'ambient' background conditions in the Gulf of Cadiz into which the salty Mediterranean water is being injected. The difference between these profiles shows the influence of the Mediterranean outflow. At station 97, the Mediterranean outflow is identifiable by its characteristic high salinity signature and can be seen confined to the bottom $130 \mathrm{~m}$ of the water column. The outflow also corresponds to relatively warm water (about $12.8^{\circ} \mathrm{C}$ ). The uppermost $\mathrm{T}, \mathrm{S}$ anomaly (or the temperature minimum) is at $620 \mathrm{~m}$ just above the large density gradient that defines the top of the Mediterranean outflow. The minimum salinity at station 97 , however, is at $560 \mathrm{~m}$ depth. The top of outflowing Mediterranean water, based on the high salinity and temperature at the bottom of CTD 97, begins $60 \mathrm{~m}$ below the salinity minimum but the depth of the top of the T,S anomaly does closely correspond to the depth where the temperature becomes a relative minimum. Therefore, defining the outflow to extend from the bottom up to the salinity minimum would give an overestimate. For transport estimates, the water column is broken up into layers, below and above the reference level. Using the salinity minimum as a level of no motion would overestimate the outflow transport and underestimate the inflow transport because the velocity vertical shear is positive. 


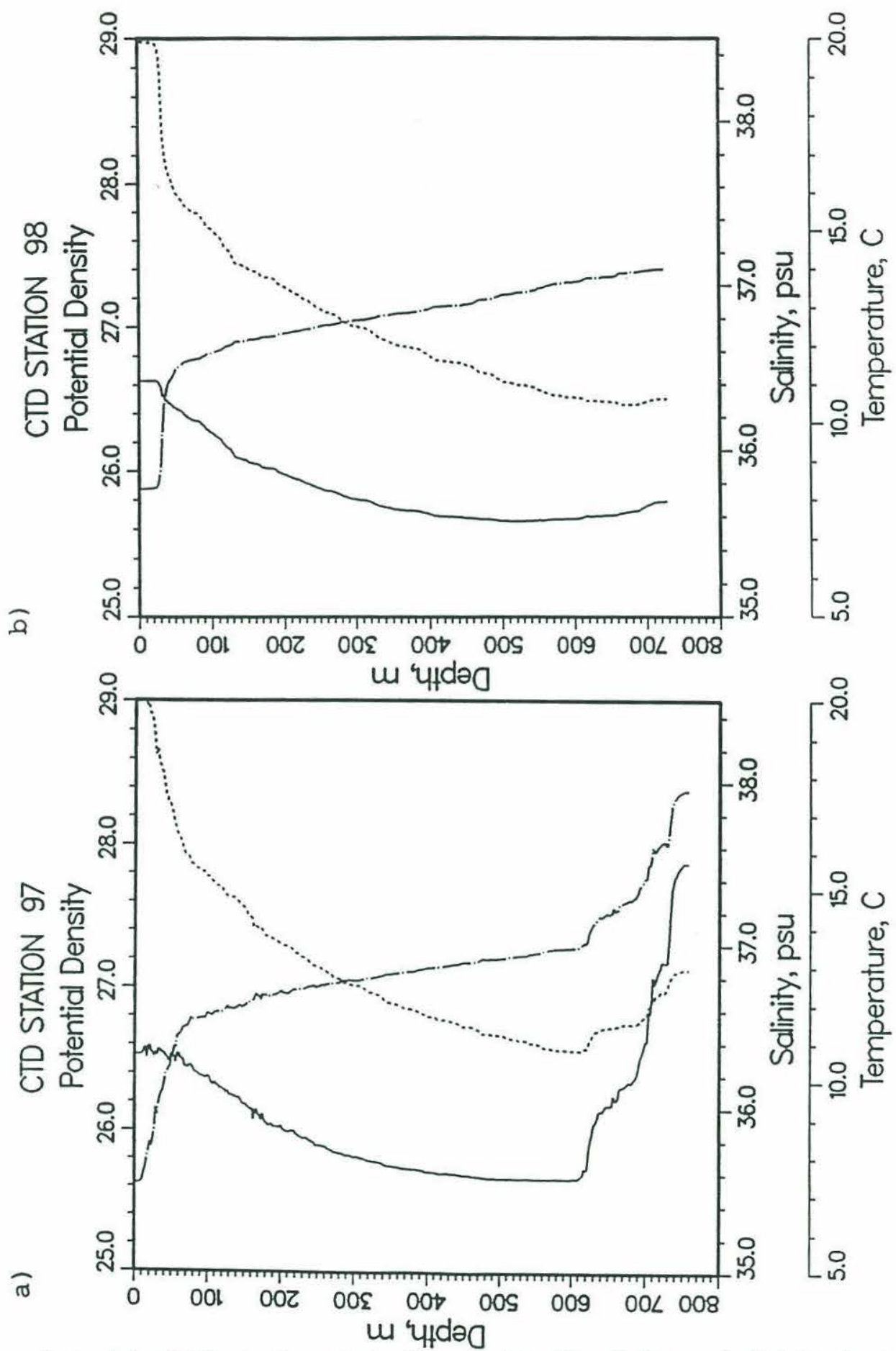

Figure A.1: (a) CTD station 97 in Section E. The Salinity (solid line) at station 97 shows salty Mediterranean water confined to the bottom $130 \mathrm{~m}$. The Temperature (dashed line) has a relative minimum near $560 \mathrm{~m}$. (b) CTD station 98 in Section E only $6 \mathrm{~km}$ away from CTD 97 contains no salty Mediterranean water. 
Figure 2.13(e) shows the T,S structure across an entire section and illustrates the limit of Mediterranean outflow influence at the $(\mathrm{T}, \mathrm{S})$ minimum. The Mediterranean outflow is again distinguished by its high salinity near the bottom ( $\sigma_{\theta}$ contours above 27.5). The extent of the Mediterranean outflow changes the temperature/salinity relation below the temperature minimum (near $\sigma_{\theta} 27.2$ ) and not further up in the water column at the salinity minimum. Note that this section has a complicated T/S structure which varies considerably across the section. These features will be discussed in section 2.4. This figure further illustrates that the top of the Mediterranean outflow cannot be defined by a single $\sigma_{\theta}$ reference surface. The deeper stations have lower temperatures at the temperature minimum and thus higher $\sigma_{\theta}$ values.

\section{A.2 Why use a reference velocity at the temper- ature minimum?}

The top of the T,S anomaly described above defines the reference level used and the second step in our referencing scheme determines the velocity at this depth. Note that this is not a classical reference 'level' because the T,S minimum is not a function of density or depth. Why isn't this a good level of no motion (i.e. why do we need a reference velocity?)? We saw in section 2.2 that the Mediterranean is a large reservoir that produces very salty water through evaporation. In the Strait of Gibraltar all the water (inflow and outflow) has salinity greater than 36.1 and temperature greater than $12.8^{\circ} \mathrm{C}$. Therefore, the low salinity and low temperature water at the temperature minimum, as shown for example in figure 2.13(e) above, could not have originated from the Mediterranean and the velocity at the temperature

minimum must be either zero or directed into the Mediterranean (we define eastward flow into the Mediterranean as positive and outflow or westward flow as negative). 
If the velocity at this minimum temperature depth were zero then we would expect the temperature of this water to slowly increase as vertical diffusion smooths the temperature profile. To maintain the temperature minimum there needs to be a source of cold water. It cannot come from the Mediterranean, therefore, this cold water must be coming from the open North Atlantic and the velocity at this depth should be positive (inward). Further, two-layer exchange flow with entrainment and diffusion will develop smooth gradients in the velocity and density between the two layers over a finite thickness (Brown and Rosko, 1976). The level of no motion between these opposing flows will lie somewhere within this gradient layer and not at either extreme. Thus, like the salinity minimum, the temperature minimum is also an upper limit to the true level of no motion because $\frac{\partial u}{\partial z}>0$.

\section{A.3 Conserving salt flux}

We could try to find the true depth of no motion for each station below the temperature minimum, but we choose instead to specify the velocity at this depth. We assume the current is steady and a closed section isolating the Mediterranean should have no net salt flux across it. The absolute velocity, $u$ is defined as,

$$
u=u_{\text {ref }}+u_{\text {shear }}
$$

the sum of a reference velocity (barotropic velocity), $u_{\text {ref }}$ and a baroclinic velocity, $u_{\text {shear }}$. The baroclinic velocity is given by the shear from the XCPs and geostrophic calculations referenced to zero at the temperature minimum. The no salt flux (NSF) condition is written as a constraint on the reference velocity, $u_{r e f}$.

$$
u_{\text {ref }}=-\frac{\iint_{A} S u_{\text {shear }} d A}{\iint_{A} S d A} .
$$

Applying $u_{\text {ref }}$ at each station at the depth of the temperature minimum is the final step in the scheme employed in this analysis to give us an absolute velocity 
field. Note that $u_{\text {ref }}$ was not applied at sections FE, F, G and $\mathrm{H}$ where it suggested westward flow away form the Mediterranean at the temperature minimum. As discussed previously, the temperature and/or the salinity minimum is the upper limit for a depth of no motion and the fresh NACW just above this depth cannot be moving westward. Figure 2.8 shows the absolute velocity field from the XCP profiles and figure 2.9) shows the absolute flow field calculated using the geostrophic relationship. Aside from assuming that the current is steady, this constraint also assumes that the entire return circulation within any section has been completely sampled (i.e. that a section is 'closed'). The Cadiz Expedition sections do not span the entire Gulf of Cadiz so this 'closed' assumption needs some justification.

\section{A.4 Justification for conserving salt flux}

Some confidence in this 'closed' assumption can be gained from inspection of Ochoa and Bray's (1991) closed sections from the Gibraltar Experiment collected in the Gulf of Cadiz in the spring and fall of 1986. Ochoa and Bray first introduced this NSF scheme which they applied at a specified steric anomaly. They compared different velocity fields calculated by imposing various constraints on the reference velocity. A common constraint on all their velocity fields was the assumption that the Mediterranean does not on average, gain or lose salt (as described above).

Figure A.2 is one of Ochoa and Bray's (1991) velocity sections along a N/S section at $6.5^{\circ} \mathrm{W}$. The reference velocity applied was that required to satisfy the above constraint. In spring and fall, the westward Mediterranean outflow (hatched contours) lies between 300-500 dbar banked against the continental slope in the northern part of the Gulf of Cadiz. During the fall, the largest, most intense eastward circulation is a surface jet that is located above the Mediterranean outflow and between the 300 and $100 \mathrm{~m}$ isobaths near the shelf break. During the spring, Ochoa and Bray found 

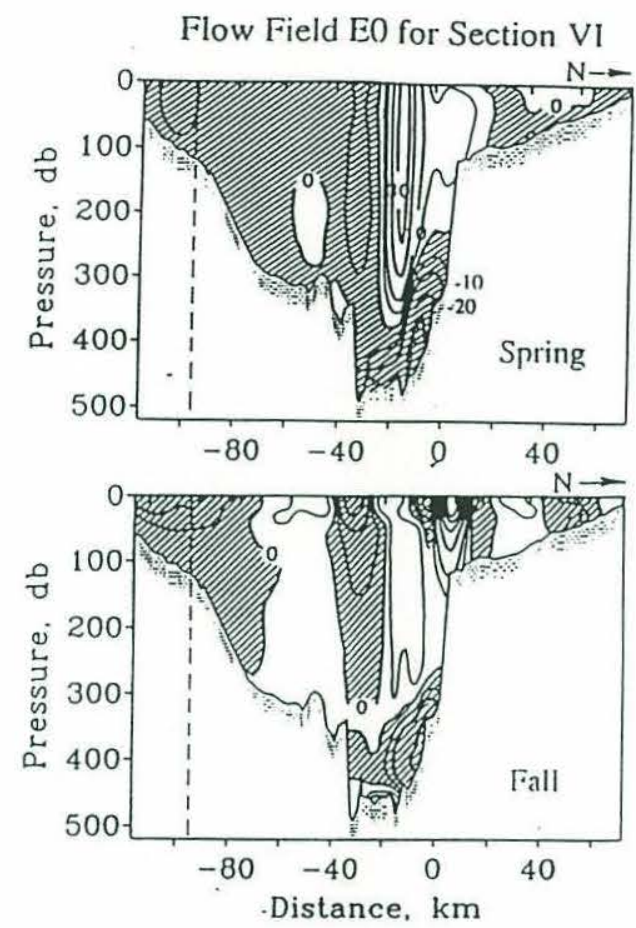

Figure A.2: Geostrophic Velocity section from Ochoa and Bray 1991. Top figure is their section VI taken during the Spring of 1986. The lower figure is the same section during the fall of 1986 .

a broad eastward current above the Mediterranean outflow, but the strong surface eastward jet is much further offshore near the $450 \mathrm{~m}$ isobath (figure A.2). Although they did not find significant seasonal variability in the transport of the outflow or the inflow, the location of the most intense eastward circulation does change. In general they found the bulk of the eastward flow was located directly above the outflow.

Ochoa and Bray's section VI can be compared with the geostrophic velocities we obtain at section D (figure 2.9(b)). Striking similarities appear which suggest that the outflow is a very stable feature since Ochoa and Bray's sections were taken two years before the Gulf of Cadiz Expedition. Our section D corresponds most 
closely with Ochoa and Bray's fall section and was also obtained in the fall. Section D also contains sluggish inflow with speeds greater than $8.5 \mathrm{~cm} / \mathrm{sec}$ directly above the westward moving Mediterranean outflow which is confined against the bottom between 300-600 dbar. The strongest inflow circulation is found as a surface jet between the $200-300 \mathrm{~m}$ isobath.

Section D only extends to the $200 \mathrm{~m}$ isobath, however, so it is possible that the Cadiz Expedition didn't fully sample the inflow circulation especially if the inflow flow was concentrated above the $200 \mathrm{~m}$ isobath. As sampled, the jet in section D represents approximately $0.2 \mathrm{~Sv}$ of the inflow circulation. This transport is a small fraction of the $1 \mathrm{~Sv}$ inflow flow across the entire section. If we sampled only half of the jet, it would imply an upper bound on the undersampling of this inflow transport by $0.2 \mathrm{~Sv}$. If the inflow is not fully sampled, then our NSF scheme would underestimate the salty outflow and overestimate the inflow directly above the outflow by requiring a large positive velocity at the temperature minimum. Therefore, even at this section, the flux of Mediterranean outflow would be underestimated by at most $0.2 \mathrm{~Sv}$. Since the bulk of the inflow circulation was directly above the Mediterranean outflow which is consistent with Ochoa and Bray (1991), it is reasonable to assume that the more limited sections in the Gulf of Cadiz Expedition adequately sampled the inflow circulation.

Based on the presence of sand waves near $6.25^{\circ} \mathrm{W}$, Kenyon and Belderson (1973) support the existence of the North Atlantic inflow circulation on the shelf (above the $70 \mathrm{~m}$ isobath). If an unsampled inflow circulation exists, then the net transport across this section certainly would not conserve total salt flux. However, the flux of Mediterranean water across any section must be conserved downstream (assuming that there is no net gain in outflow mass between sections). This is just a statement of conserving the mass of the outflow. Rather than applying the continuity of salt flux directly, the flux of salty Mediterranean water has been conserved 


\begin{tabular}{|c|c|c|c|c|}
\hline Section & $\begin{array}{c}\text { Net } \\
\text { Transport } \\
\text { Sv }\end{array}$ & $\begin{array}{c}\text { Heat } \\
\text { Flux } \\
10^{12} W\end{array}$ & $\begin{array}{c}\text { E-P } \\
c m y r^{-1}\end{array}$ & $\begin{array}{c}\text { Heat } \\
\text { Loss } \\
W m^{-2}\end{array}$ \\
\hline A & 0.043 & 8.1 & 54 & -3.2 \\
\hline B & 0.033 & 5.1 & 42 & -2.0 \\
\hline $\mathrm{C}$ & 0.023 & 5.3 & 29 & -2.1 \\
\hline D & 0.022 & 0.9 & 28 & -0.4 \\
\hline $\mathrm{E}$ & 0.032 & -8.6 & 40 & 3.4 \\
\hline $\mathrm{F}$ & 0.029 & 8.8 & 37 & -3.5 \\
\hline
\end{tabular}

Table A.1: Net volume and heat flux for each XCP section. The reference level used here is the T,S minimum with the zero net salt flux described in section 2.3. The inferred E-P and heat loss assumes the area of the Mediterranean is $2.5 \cdot 10^{6} \mathrm{~km}^{2}$.Positive fluxes are directed into the Mediterranean.

indirectly by requiring no net salt flux. Table 2.3 shows how the flux of salt has been conserved downstream. The flux of Mediterranean water will be discussed further in section 2.5.

\section{A.4.1 Results}

The referencing technique used here, results in a surprising degree of consistency among all the sections, indicating the total mass flux for all the sections is very small and directed into the Mediterranean. The estimate of net mass flux into the Mediterranean is consistent with excess evaporation over the Mediterranean, i.e. is approximately the flux required to balance evaporation. The estimate of net mass flux across sections A through $\mathrm{F}$ is given in Table A.1 and ranges between $.022-.043$ Sv. Assuming that the evaporation takes place over a surface area of $2.5 \times 10^{6} \mathrm{~km}^{2}$, implies evaporation rates which range from $28-54 \mathrm{~cm} \mathrm{yr}^{-1}$ and which is close to the Bryden et al.(1989) estimate of $55 \mathrm{~cm} \mathrm{yr}^{-1}$. Similarly, the net heat transport yields estimates of average heat loss the same order as Ochoa and Bray (1991) estimate of $2-6 \mathrm{~W} \mathrm{~m}^{-2}$, although at section $\mathrm{E}$ the heat flux is in the wrong direction. 
Section transport $87-81$

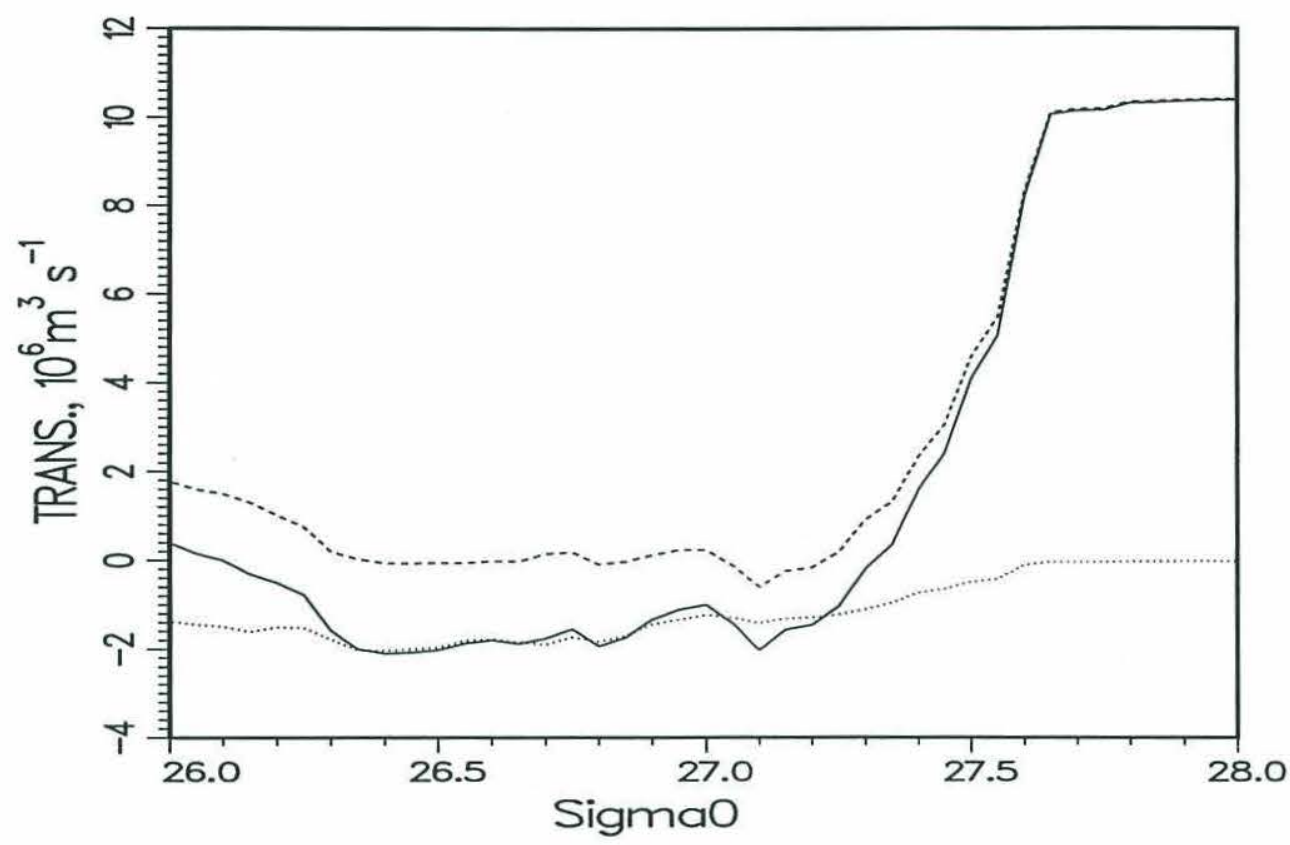

Figure A.3: Section D net transport of Mediterranean outflow (solid line), the outflow transport(dotted line) and surface water (dashed line) as a function of the zero velocity $\sigma_{\theta}$ reference surface.

\section{A.5 Sensitivity to reference level}

Using the NSF velocity at each temperature minimum depth, the flux of Mediterranean salt is approximately conserved downstream and the net mass flux into the Mediterranean approximately balances the evaporation inside the Mediterranean basin. One way to examine the uncertainty in the transport estimates is to consider the sensitivity of the transport estimates to the choice of reference level. In figure A.3, the total outflow transport across section $\mathrm{D}$ is calculated for various $\sigma_{\theta}$ reference level surfaces. Section D is presented here because it reproduces features seen in all sections and the reader should now be very familiar with its absolute velocity field. In figure A.3 the solid line represents the transport of Mediterranean 
water westward and the dashed line is the inflow transport from the North Atlantic. The solid line is the net transport across the section. For this section, the minimum salinity at each station lies in the range of 27.04 to $27.22 \sigma_{\theta}$ : i.e. the salinity minimum for each station does not lie along the same $\sigma_{\theta}$ surface or depth (see figure 2.13(b)). Therefore, varying the $\sigma_{\theta}$ level of no motion does not precisely measure the effect of adjusting the salinity minimum depths up and down through the water column, but rather demonstrates the sensitivity of the transport to different reference levels in general. Reasonable values for $\sigma_{\theta}$ levels of no motion lie slightly deeper than the temperature minimum within the range of 27.2 to $27.35 \sigma_{\theta}$. Within this range, the outflow transport ranges from $-1.29 \mathrm{~Sv}$ to $-0.96 \mathrm{~Sv}$ east while the inflow transport varies from $-0.21 \mathrm{~Sv}$ east to $+1.3 \mathrm{~Sv}$ west (figure A.3). Averaging these numbers, the outflow transport can be approximated at $1.1 \pm .2 \mathrm{~Sv}$ while the inflow is approximately $0.6 \pm 0.8 \mathrm{~Sv}$. The maximum vertical shear appears just below the temperature minimum. The inflow transport is very sensitive to the level of no motion while the outflow transport is not, implying that the outflow has high shear throughout its depth and that there is very little shear above the salinity minimum through the upper portion of the water column. The inflow transport depends sensitively upon how much shear is placed above a level of no motion. The inflow transport varies by as much as $1.5 \mathrm{~Sv}$ while the outflow transport estimates for all sections vary within $0.2 \mathrm{~Sv}$.

\section{A.6 Comparison with historical salinity minimum reference level}

Historically, the depth of the salinity minimum has been used as the level of no motion for the Mediterranean outflow (Ambar and Howe 1979a, Zenk 1975a). The depth of the salinity minimum is an upper limit to the actual level of no motion and 
transport estimates based on no motion at the depth of the salinity minimum overestimate the Mediterranean outflow transport and underestimate the surface inflow transport. For instance, the salinity minimum reference level gives a net transport above the outflow (which we define as the inflow) of $.55 \mathrm{~Sv}$ moving to the west at section $\mathrm{E}$, in the same direction as the outflow. Through section F, the salinity minimum reference gives $3.0 \mathrm{~Sv}$ inflow moving east. This is a $3.6 \mathrm{~Sv}$ mass imbalance above the outflow between these section. At the same time, the outflow transport increases from $1.46 \mathrm{~Sv}$ to $2.3 \mathrm{~Sv}$ between sections $\mathrm{E}$ and $\mathrm{F}$. Even assuming a $1 \mathrm{~Sv}$ vertical exchange (or entrainment) between the inflow and outflow layers, which can't be justified by the water properties, there would still be a mass imbalance of $2.8 \mathrm{~Sv}$. There is only $0.2 \mathrm{~Sv}$ 'leakage' of salty outflow across section $\mathrm{FE}$ which also can't reduce this mass imbalance significantly. Using the temperature minimum NSF scheme, however, the net mass imbalance, including section $\mathrm{FE}$, is reduced form $2.8 \mathrm{~Sv}$ to only $0.3 \mathrm{~Sv}$ moving west across section $\mathrm{F}$, well within our expectations of accuracy and sampling bias.

\section{A.7 Summary and comments}

In this appendix, the velocity referencing scheme that is employed for this data was described. By referencing the geostrophic shear and XCP shear at the temperature minimum and then requiring the salt flux to be conserved for the sections close

to the strait, we have reduced mass imbalances between sections and approximately conserved the flux of Mediterranean water.

The only draw back of this referencing scheme is that we only approximately conserve the flux of 'pure' Mediterranean water downstream through all our sections (in section 2.5.1 we find that section A and F contained too much 'pure' Mediter- 
ranean water). We would like to conserve this quantity as well, ${ }^{1}$ but to do so requires that we adjust all station reference levels simultaneously (i.e. an inverse). We cannot adjust in isolation the reference levels at section $\mathrm{A}$ because lowering the reference level through the shear (which you must do to decrease the outflow transport) produces a large mass imbalance through section A. For instance to reduce the flux of 'pure' Mediterranean water (see section 2.5.1) to $0.4 \mathrm{~Sv}$ requires reducing the outflow transport to $0.53 \mathrm{~Sv}$ which leads to an inflow transport of $1.2 \mathrm{~Sv}$, a net flux of 0.7 Sv into the Mediterranean. This is essentially the same result shown in figure A.3, namely that the inflow transport is most sensitive to the reference level.

\footnotetext{
${ }^{1}$ It is unclear even if we should expect to conserve the flux of 'pure' Mediterranean water or the net salt flux. If we only know the flow to $\pm 20 \%$ as Armi and Farmer (1988) suggest then we shouldn't expect to exactly conserve either of these quantities exactly, which makes the error estimates in tappered least square inverses more appealing.
} 


\section{Appendix B}

\section{What is the residual of the}

\section{downstream momentum budget?}

The stress estimated by the method described in chapter 2.6 is considered to be the total stress which includes both the bottom and the interfacial stress. What is estimated from this momentum budget residual is of considerable importance and is also fairly ambiguous, so we will examine the subtlety of this question by revisiting the derivation of equation 2.13 .

Commonly, the downstream divergence of the Bernoulli potential is considered to be balanced by the stress divergence, $\partial \tau / \partial z$. On the other hand, here the total stress, $\tau$, is being estimated by the divergence of the integrated momentum flux which is not strictly speaking a Bernoulli potential. The momentum equation has been integrated and the continuity equation has been added, so we are really calculating the residual of a transport equation. 
Let us consider each term in the momentum equation separately. Integrating the acceleration term we have,

$\int_{-l}^{+l} \int_{-D}^{-D+h} \partial_{\zeta}\left(u^{2}\right)+\partial_{\eta}(u v)+\partial_{z}(u w) d z d \eta=\iint_{A} \partial_{\zeta}\left(u^{2}\right) d A+\left.\int u v d z\right|_{-l} ^{+l}+\left.\int u w d \eta\right|_{-D} ^{-D+h}$.

Application of Leibnitz's rule and assuming that horizontal entrainment is negligible so that the edges of the flow are material surfaces ${ }^{1}$ then,

$$
v( \pm l)= \pm u \partial_{\zeta} l
$$

If we allow entrainment across the upper layer interface, then the interface $z=-D+h$, is not a material surface. This means that,

$$
w(-D+h)=u \partial_{\zeta}(-D+h)+w_{*}
$$

where $w_{*}$ is the entrainment velocity across the interface. The integrated acceleration term then simplifies to

$$
\partial_{\zeta} \iint\left(u^{2}\right) d z d \eta+\left.\int u w_{*} d \eta\right|_{-D+h}
$$

The last term of this equation involves the entrainment velocity $w_{*}$ and certainly contains the character of interfacial stress. In fact, in layered models the interfacial stress term in the momentum equation is parameterized as $\rho_{o}\left(u-u_{a b o v e}\right) w_{*}$. In this case however, $u(-D+h)$ is defined to be zero (it is a level of no motion) and this entrainment-like term will not alter the total stress estimate (equation 2.13).

If this were a layered model with uniform velocity, $\bar{u}$ and $\rho$ and the layer above had velocity $u_{a b o v e}$ then the continuity equation would be

$$
\partial_{\zeta} \bar{u} \bar{h} \text { Width }=-w_{*} \text { Width }
$$

\footnotetext{
${ }^{1}$ Note that this approximation is less strict and more accurate than Smith's assumption that $L_{\eta} \gg L_{\zeta}$. We've seen that the initial width of the flow is $\approx 15 \mathrm{~km}$ and downstream changes occur over the same length scale. We will also find that $w_{*}$ through the top of the interface is small and that horizontal entrainment is likely $\ll u \partial_{\zeta} l$.
} 
Also, the acceleration term integrates as

$$
\begin{gathered}
\partial_{\zeta} \bar{u}^{2} \bar{h} \text { Width }+w_{*} u_{\text {above }} \text { Width } \\
=\bar{u} \bar{h} \text { Width } \partial_{\zeta} \bar{u}+w_{*} \text { Width }\left(u_{\text {above }}-\bar{u}\right)
\end{gathered}
$$

Thus you can see that the interfacial "stress" term $\rho_{o}\left(u-u_{a b o v e}\right) w_{*}$ arises from the acceleration term. If the flow above, $u_{\text {above }}$ were motionless the momentum transport equation of the layered model would only be modified by the integral of the stress divergence which would include only the bottom stress because the layer above is assumed to have no stresses acting upon it. If the flow above is not motionless, however, the flow momentum would be reduced by the $u_{a b o v e} w_{*}$ term.

The integrated stress term is unambiguously

$$
\iint_{-D}^{-D+h} \partial_{z} \tau d z d \eta=-\int \tau_{I}+\tau_{B} d \eta
$$

where $\tau_{I}$ and $\tau_{B}$ have been defined to oppose the mean motion. $\tau_{B}$ is the bottom stress and $\tau_{I}$ is the stress at the surface, $z=-D+h$ where $u=0$. Since we define $z=-D+h$ as the interface between the Mediterranean water and the NACW above it is convenient to call $\tau_{I}$ the interfacial stress.

Let us consider the idealized velocity profile shown in figure B.1. The alongstream velocity profile shows eastward (positive) flow at the top of the water column and westward (negative) flow representing the Mediterranean outflow at the bottom of the water column. Note that this idealized velocity profile illustrates the general character of the actual velocity profiles taken in the Gulf of Cadiz. The density profile shows the strong density gradient 'capping' the outflow. If we assume that the stress on the flow is proportional to $\partial u / \partial z$ this velocity profile would have a stress profile similar to the sketch in figure B.1. The stress at the bottom of the profile is $\tau_{B}$ and the stress decreases to a minimum of $\tau_{I}$. Above, we define $\tau_{I}$ as the stress at the top of the interface where the along-stream velocity is zero, but this $\tau_{I}$ does not vanish 


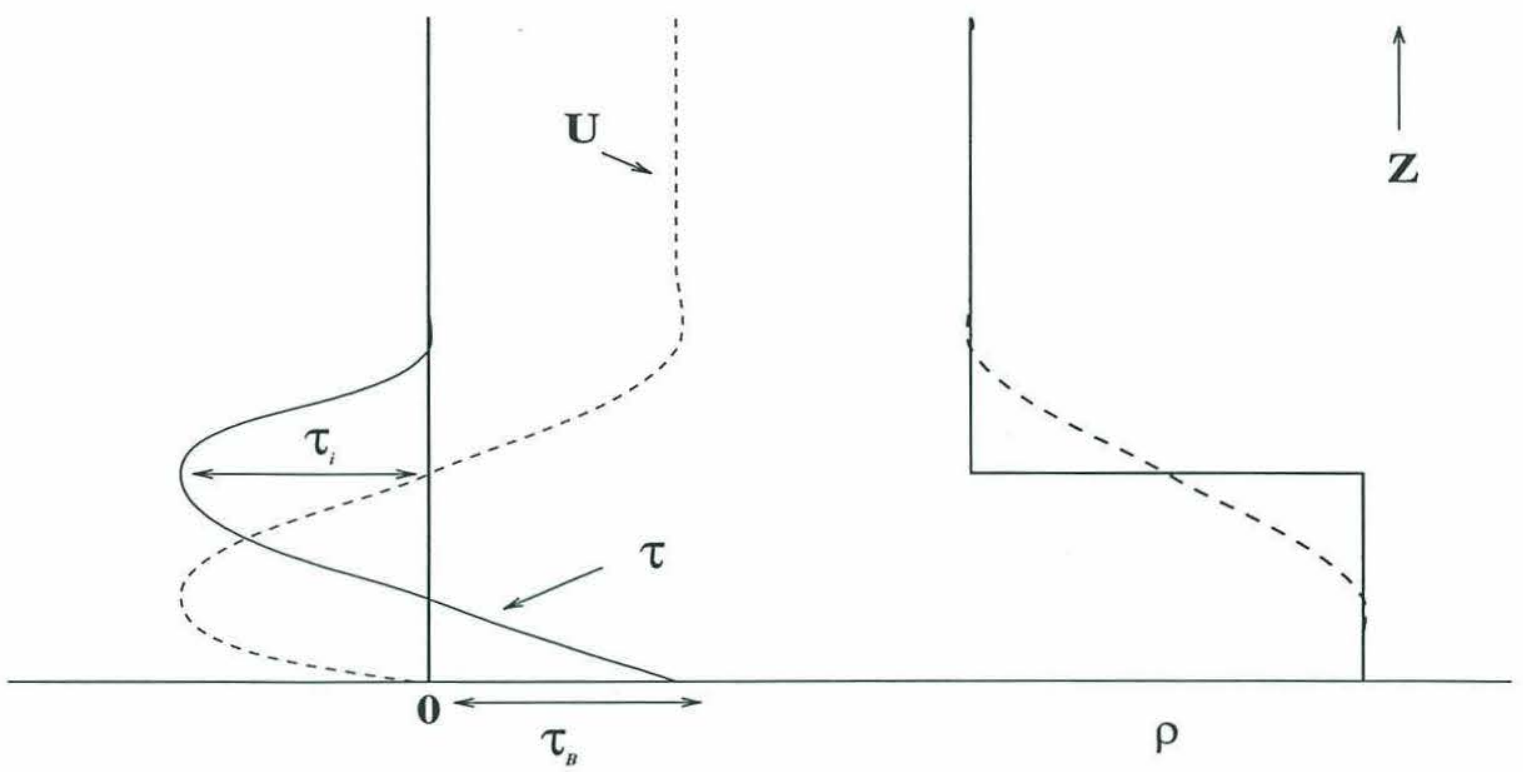

Figure B.1: Idealized schematic of a typical velocity profile. (a) The along-stream velocity (solid line) shows a sheared profile. A possible stress profile is sketched (dashed line). (b) Density of the flow showing two layers separated by a large density gradient.

there since the flow above is not motionless. Therefore, the residual of the transport equation is the total stress including bottom and interfacial stress (as well as small scale unreasolved pressure fluctuations). 


\section{Appendix $\mathbf{C}$}

\section{Layered Pressure Gradient}

Here, we derive the pressure gradient of a layered model where the lowest layer density is assumed to be vertically uniform but can vary in the horizontal. Namely, we assume that $\rho_{2}=\rho_{2}(x, y)$. The upper layer has uniform density, $\rho_{1}$ and is assumed to be motionless. The schematic of the layer configuration is shown in figure C.1, where $h(x, y)$ is the height of the lowest layer and the bottom depth is given by $D(x, y)$.

The pressure at the interface, $p\left(z_{i}(x, y)\right)$, and in the lowest layer, $p_{2}(x, y, z)$, are given by

$$
\begin{aligned}
p\left(z_{i}\right) & =-\rho_{1} g z_{i}+p(z=0) \\
& =-\rho_{1} g(-D+h)+p_{\circ}
\end{aligned}
$$

where $p(z=0)=p_{o}$.

$$
p_{2}(x, y, z)=p\left(z_{i}\right)-\rho_{2}(x, y) g\left(z-z_{i}\right)
$$

The pressure gradient in the lowest layer is then given by

$$
\nabla p_{2}(x, y, z)=\nabla\left(\rho_{2}-\rho_{1}\right) g(-D+h)-\nabla \rho_{2} g z+\nabla p_{\circ}
$$


Define $\delta \rho=\rho_{2}-\rho_{1}$ and integrate equation C.2 vertically through the vertically uniform lower layer yields,

$$
\begin{aligned}
\int \nabla p_{2} d z & =h \nabla(\delta \rho g(-D+h))-g\left(\frac{1}{2} h^{2}-h D\right) \nabla \rho_{2}+h \nabla p_{\circ} \\
& =-g \delta \rho h \nabla D+\nabla\left(\frac{1}{2} g h^{2} \delta \rho\right)+h \nabla p_{\circ}
\end{aligned}
$$

Note that the more familiar form of a layered pressure gradient is $g \delta \rho h \nabla(-D+$ $h$ ) which is valid when both layers are of uniform density. When you allow the lowest layer density to change you must account for the gradient in the internal pressure gradient term.

\section{Streamtube Pressure Gradient}

The simple plume model described in chapter 3 is derived following Smith (1973) with only a change in the entrainment and friction parameterizations. The model represents a 'stream-tube' which is defined as a tube of fluid bounded by a contour on

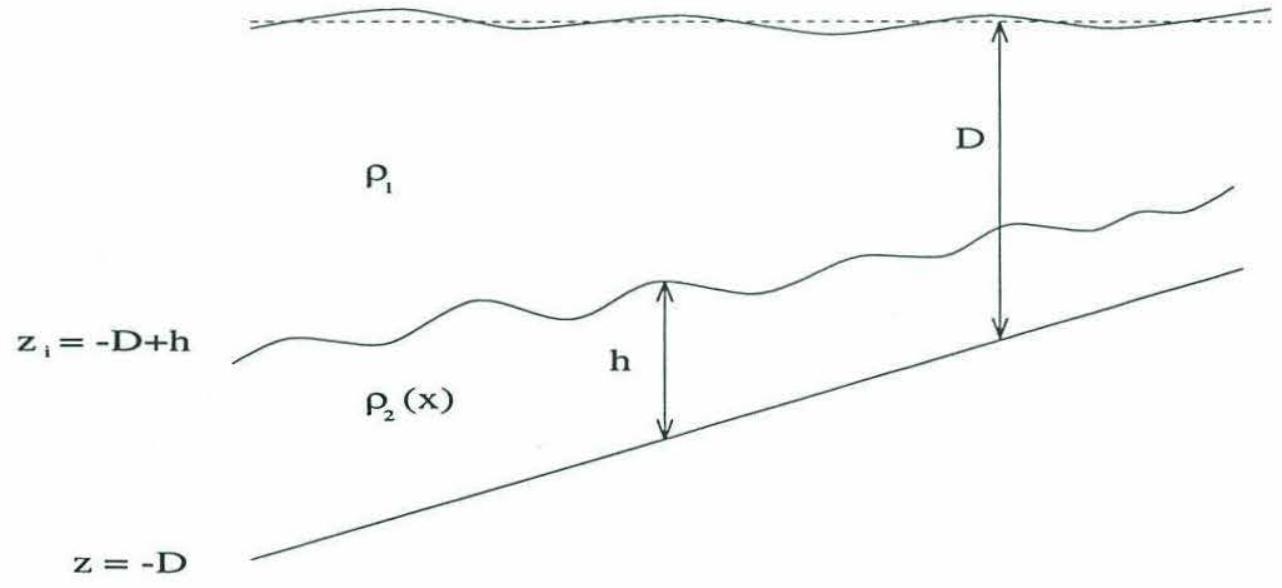

Figure C.1: Schematic of two layer model showing the appropriate parameters to determine the pressure gradient of the lowest layer. The total water depth is D and the height of the lowest layer is h. The top layer has uniform density, $\rho_{1}$, and the lowest layer that has vertically uniform density that is a function of horizontal position, $\rho_{2}(x, y)$. 
which $\rho^{\prime}$ and $\mathrm{u}=0$. The integral equations are also derived in Chapter 2, section 2.6.1 and section 2.6 .3 and discussed in section 2.6.3. The adaptation to a layered form assumes that you can define average quantities across the section. This is one of the most tenuous assumptions in the model.

For instance, the pressure gradient is integrated over a cross section of the outflow so that at each location downstream the whole layer is forced as a single point at the mid-point of the cross section. Thus the model variables can be thought of as average horizontal integrals. For instance, $h_{\text {model }}=\int h d y / \int d y$, where y locally normal to streamlines and $y=0$ is the mid-point of the cross section.

Technically, Smith (1973) assumes that the outflow is sufficiently thin that $\frac{\partial \rho_{a m b}}{\partial z} h \ll \rho^{\prime}$ and vertical variations in the ambient density profile can be ignored ${ }^{1}$. We also assume that $\frac{\partial \rho_{a m b}}{\partial z} \alpha W / 2 \ll \rho^{\prime}$ and the density constrast of the layer can be evaluated at the 'stream-axis' or mid-point of a cross section (Smith 1973). If the background has constant stratification these retrictions can be relaxed slightly by noting that the integration of the density anomaly reduces to the density anomaly at the mid-point (and mid-layer-depth). For instance, if the background stratification is given by $\frac{\partial \rho_{a m b}}{\partial z}$ and the interface $z_{i}$ has a constant slope, $\alpha_{i}$ then the density anomaly can be expressed as

$$
\delta \rho=\delta \rho(y=0)+\alpha_{i} \frac{\partial \rho_{a m b}}{\partial z} y
$$

where $y+$ is towards shallower and therefore lighter ambient density (i.e. increasing the buoyancy anomaly). Integrating in y to the limits of the flow at $y= \pm L$, the effect of the variable stratification vanishes and the density anomaly of the layer can be exactly specified by the density anomaly at the mid-point of the cross section.

\footnotetext{
${ }^{1}$ note that the ambient profile sets the hydrostatic pressure gradient which is removed before integration
} 


\section{Application in the Simple Plume Model}

In the simple model described in chapter 3 , we have assumed that the upper layer is motionless (see also section 2.6.3). Therefore, $\nabla p_{o}$ is identically zero. We have further assumed that

$$
g \delta \rho h \nabla D \gg \nabla\left(\frac{1}{2} g h^{2} \delta \rho\right)
$$

which effectively says we are only interested in the dynamics of flow on a slope. Given that the depth of the Mediterranean outflow changes by $1000 \mathrm{~m}$ and the density anomaly changes by $1 \mathrm{~kg} / \mathrm{m}^{3}$, and assuming the height remains near $100 \mathrm{~m}$ and the average density anomaly is $1 / 2 \mathrm{~kg} / \mathrm{m}^{3}$, neglecting the internal pressure gradient is equivalent to saying $10^{5} \gg 10^{4}$. In fact, the decrease in density downstream is partially compensated by the increase in height downstream and the internal pressure gradient is even smaller (and this scaling is even smaller). Over the whole path of the flow the internal pressure gradient term appears to be small, but we saw in Chapter 2 that the density is eroded over a very small portion of the path of the outflow and therefore this term could be locally important (and in fact is according to figure 2.25, discussed in this section).

In neglecting this term, we have assumed that the interface slope parallels the bottom topography. Through visual inspection of the property sections, we could conclude that this approximation is reasonable near the center of the outflow, but is certainly invalid where the contours of density intersect the bottom near the edges of the flow. Therefore, this model cannot easily describe the spreading of the flow which is intimately tied to the detailed fate of the interface where it intersects the bottom. This approximation also filters gravity waves and thus eliminates the hydraulic nature of the flow and there can be no upstream influence.

We would like to include this term explicitly. We have made some attempts to include this term by iterating the model equations in a form of Euler forward 
estimation. This has not met with success. The problem arises from our chosen solution technique. Without this term we have a purely hyperbolic problem that lends itself easily to following a particle through time, specifying only the initial conditions. Adding this term would mean including upstream influence which would make us unable to integrated forward in this manner. Including this term would necessitate a higher dimensional model where the full structure is evaluated.

The effect of this term is to accelerate the flow in the direction the interface layer thins and towards regions of lower density anomaly and therefore lower pressure. Any attempt to include this term while maintaining the lagrangian solution technique is inherently unstable. Figure $3.9 \mathrm{a}$ and $3.7 \mathrm{~b}$ show that both the density anomaly and the layer height decrease strongly at the beginning of the model integration. The hydraulic pressure term could therefore be important initially. In the Strait for example the flow is driven almost entirely by this internal pressure gradient. Unfortunately, the direct inclusion of this additional forcing term in the model will accelerate the flow downstream which will further thin the outflow layer through continuity and increase the mixing by raising the Froude number which will further reduce the density anomaly. However, if internal waves were allowed to propagate upstream any rapid adjustment of the interface would be reduced by adjusting the upstream conditions.

A simple example is illuminating. Suppose that we have a model solution as shown in figure C.2 where, following the flow, the flow decelerates and thickens. From one time step to another, our model would allow this change in thickness to be arbitrarily steep. If the model included the internal pressure gradient and allowed internal gravity waves to influence the upstream statez, the interface height change would not be arbitrarily steep, but would geostrophically adjust to some smoothed profile $^{2}$ (shown as a dashed line in figure C.1). The internal pressure gradient term

\footnotetext{
${ }^{2}$ This is not true if the flow were critically controlled and gravity waves could not travel upstream.
} 


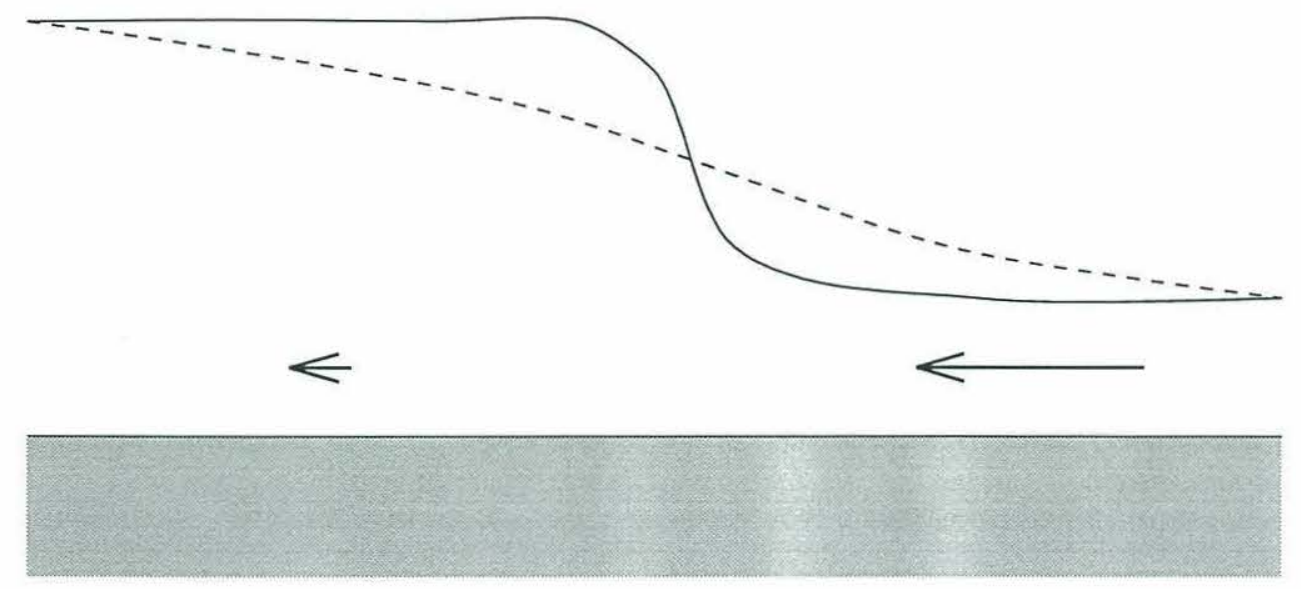

Figure C.2: Schematic of layered interface steepening. The velocity at two different times as shown with arrows. The solid line represents the interface height hypothetically calculated from the simple model described in chapter 3 . The dashed line represents the smoothed interface height you might expect if the interface were allowed to adjust. The flow is shown to decelerate and thicken as it moves downstream, similar to what might be expected for a column of fluid taking a cycloid path.

implied by these two different interface configurations are very different. The abrupt interface steepening would generate an internal pressure gradient forcing to rapidly and very locally decelerate the flow. Thus, the internal pressure gradient has a positive feedback on the velocity. The gradual steepening of the interface also acts to decelerate the flow and is felt further upstream and downstream. The upstream influence that this internal pressure gradient term implies is the reason why including this term is incompatible with integrating downstream following the fluid.

The deceleration and thickening shown here is evident in the inertial oscillations observed in the model solutions discussed in chapter 3. As the flow turns and begins to slide up the slope again, the flow decelerates and thickens by continuity. Therefore, inclusion of this internal pressure gradient term further accentuates these oscillations. Clearly, this pressure gradient term will be quite important in this process, although we might expect that if the interface slope could be correctly modeled as a gradual process, this pressure gradient would 'stiffen' the flow by decelerating 
the flow further upstream which might decrease the Coriolis force driving the inertial turn etc.

This pressure gradient term can be diagnosed from the model a posteriori, as one method for estimating the relative importance of this term. In figure 2.25 , the time integrated pressure work arising from this term is also shown relative to all the modeled terms in the energy budget. The pressure work arising from this term is calculated as

$$
\vec{u} \cdot \nabla\left(\frac{1}{2} g h^{2} \delta \rho\right) .
$$

Figure 2.25 shows that the pressure work arising from this term, like everything thing else, is most important during the initial descent of the outflow where the current thins and entrains, thereby decreasing both $h$ and $\delta \rho$. Note that the model overemphasizes this term because of the rapid thinning of the model: note in figure 3.9a that the model thins much more rapidly than the observations. The model also overemphasizes the entrainment rate (figure 3.11 ) so that the change in density of the outflow is more rapid than the observations (figure 3.8c). These two effects both contribute to a high estimate of the internal pressure gradient. Over the whole path of the flow, the net work done on the system by this term is at most $25 \%$ of the work done by the external pressure gradient term, $\vec{u} \cdot g \delta \rho h \nabla D$ (chain-dotted line in figure 2.25). Note that the oscillations described above would do no net work on the outflow and hence do not appear in the internal pressure work term. The only place where the internal pressure gradient has a significance effect on the dynamics (i.e. aside from the inertial oscillations discussed above), is near $150 \mathrm{~km}$ downstream where the bottom slope becomes small (see figure 3.10d). Although we would prefer that the magnitude of this internal pressure gradient term was vanishingly small, it appears to be of less importance than the external pressure gradient. Further, the complications of including this term increase the technical difficulties beyond warrant for such a simple model. 


\section{REFERENCES}

Ambar I. (1983) A shallow core of Mediterranean water off western Portugal. Deep Sea Research, 30, 677-680.

Ambar, I. and M. R. Howe, (1979a) Observations of the Mediterranean outflow. I. Mixing in the Mediterranean outflow. Deep Sea Research, 26A, 535-554.

Ambar, I. and M. R. Howe, (1979b) Observations of the Mediterranean outflow. II. The deep circulation in the vicinity of the Gulf of Cadiz. Deep Sea Research, 26A, 555-568.

Arhan, M., (1987) On the large scale dynamics of the Mediterranean outflow. Deep Sea Research, 34, 1187-1208.

Armi, L., and D. M. Farmer, 1986. The hydraulics of two layers with different densities. Journal of Fluid Mechanics, 163, 27-58.

Armi, L., and D. M. Farmer, 1987. Maximal two-layer exchange through a contraction with barotropic net flow. Journal of Fluid Mechanics, 164, 27-51.

Armi, L., and D. M. Farmer, 1988. The flow of Mediterranean water through the Strait of Gibraltar. Prog. Oceanog., 21, 1-106.

Armi, L. and N.A. Bray, (1982) A standard analytic curve of potential temperature versus salinity for the western North Atlantic. Journal of Physical Oceanography, 12, 384-387.

Batchelor, G. K. (1967) An Introduction to Fluid Dynamics, Cambridge University Press.

Baringer, M. O. and J. F. Price, (1990) A simple model of the descending Mediterranean outflow plume. Physical Oceanography of Sea Straits, NATO-ASI series, Kluwer Academic Publishers, ed. L. J. Pratt, 298-308.

Belderson, N. H. (1979)

Bormans, M. and C. Garrett, (1989) A simple criterion for gyre formation by the surface outflow from a strait, with application to the Alboran Sea. Journal of Geophysical Research, 94, 12637-12644.

Brass, G. W., J. R. Southam, and W. H. Peterson, 1982. Warm saline bottom water in the ancient ocean. Nature, 296, 620-623. 
Bryden, H. L., and H. M. Stommel, (1984) Limiting processes that determine basic features of the circulation in the Mediterranean Sea. Oceanologica Acta, 7, 289-296.

Bryden, H. L., E. C. Brady and R. D. Pillsbury, (1989) Flow through the Strait of Gibraltar. Seminario Sobre la Oceanografia Fisca del Estrecho de Gibraltar, Madrid, 24-28 Octubre 1988, edited by J. L. Almazan, H. Bryden, T. Kinder and G. Parrilla, SECEG, Madrid, 166-194.

Bryden, H. L. and T. L. Kinder, (1992) Steady two-layer exchange through the Strait of Gibraltar. Deep Sea Research, (in press).

Bryden, H. L., J. C. Candela and T. H. Kinder, (1993) Exchange through the Strait of Gibraltar. Progress in Oceanography, submitted.

Candela, J. C., C. D. Winant, and H. L. Bryden, (1989) Meteorologically forced subinertial flows through the Strait of Gibraltar. Journal of Geophysical Research, 94, 12667-12679.

Candela, J. C., C. Winant, and A. Ruiz, (1990) Tides in the Strait of Gibraltar. Journal of Geophysical Research, 95, C5, 7313-7335.

Candela, J. C. (1991) The Gibraltar Strait and its role in the dynamics of the Mediterranean Sea. Dynamics of Atmospheres and Oceans, 15, 267-299.

Chow, V. T., (1959) Open Channel Hydraulics, Mc Graw-Hill, 680 pp.

D'Asaro, E. (1988) Generation of submesoscale vorticies: a new mechanism. Journal of Geophysical Research, 93, 6685-6693.

Dalziel, S. B., (1988) Two-layer hrdaulics: Maximal exchange flows. PhD thesis, Department of Applied Mathematics and Theoretical Physics, University of Cambridge.

Dalziel, S. B., (1990) Rotating two-layer flows. Physical Oceanography of Sea Straits, NATO-ASI series, Kluwer Academic Publishers, ed. L. J. Pratt.

Defant, A., (1961) Physical Oceanography, Vol. I. Pergamon Press, New York, 729 pp.

Ellison, T.H. and Turner, J.S, (1959) Turbulent entrainment in stratified flows. Journal of Fluid Mechanics, 6, 423-448.

Garrett, C., R. Outerbridge and K. Thompson, (1993) Interannual Variability in Meidterranean Heat and Buoyancy Fluxes. Journal of Climate, 6, 5, 900-910. 
Goldstein, S. (1931) On the stability of superposed streams of fluids of different densities. Proc. Roy. Soc, A, 132, 524-548.

Griffiths, R. W., P. D. Killworth and M. E. Stern, (1982) Ageostrophic instability of ocean currents. Journal of Fluid Mechanics, 117, 343-377.

Griffiths R. W. (1986) Gravity currents in rotating systems. Ann. Rev. Fluid Mech., 18, 59-89.

Grundlingh, M. L., (1981) On the observation of a solitary event in the Mediterranean outflow west of Gibraltar. "Meteor" Forschungergebnisse, A, 15-46.

Hazel, P. (1972) Numerical studies of the stability of inviscid stratified shear flows. Journal of Fluid Mechanics, 51, 39-61.

Heathershaw, A. D. (1976) Measurements of turbulence in the Irish Sea benthic boundary layer. in The Benthic Boundary Layer, I. N. McCave, ed., Plenum, N. Y., 11-31.

Heezen, B. C. and G. L. Johnson, (1969) Mediterranean undercurrent and microphysiography west of Gibraltar. Bell Inst. Oceanogr., Monaco, 67 (1382), 1-97.

Hogg, N.G. and H. Stommel, (1985) On the relation between deep circulation and the Gulf Stream. Deep Sea Research, 32, 1181-1193.

Hopkins, T. S., (1978) Physical processes in the Mediterranean basins. in Estuarine Transport Processes, edited by B. Kjerve, University of South Carolina Press, Columbia, 269-310.

Howe, M. R., M. I. Abdullah, and S. Deetae (1974) An interpretation of the double $\mathrm{T}-\mathrm{S}$ maxima in the Mediterranean using chemical tracers. Journal of Marine Research, 32, 377-386.

Johnson, G. C., and T. B. Sanford, (1992) Secondary circulation in the Faroe Bank Channel Outflow. Journal of Physical Oceanography, 22, 927-933.

Johnson, G. L., T. Sanford and M. O. Baringer (1993a) Stresses in the Mediterranean Outflow: distribution of properties. Journal of Physical Oceanography, submitted.

Johnson, G. L., R. Lueck and T. Sanford (1993b) Dissipation data from the 1988 Gulf of Cadiz Expedition. Journal of Physical Oceanography, accepted.

Jones, D. W., (1989) Velocity profiler (XCP) observations of a bottom boundary layer in the Strait of Juan de Fuca. APL-UW TR 8927, Applied Physics Laboratory, University of Washington, Seattle, WA, 209 pp. 
Kantha, L. H., O. M. Phillips, and R. S. Azad, (1977) On turbulent entrainment at a stable density interface. Journal of Fluid Mechanics, 79, 753-768.

Kato, H., and O. M. Phillips, (1969) On the penetration of a turbulent layer into a stratified fluid. Journal of Fluid Mechanics, 37, 643-655.

Kase, R. H., and W. Zenk, (1987) Reconstructed Mediterranean salt lense trajectories. Journal of Physical Oceanography, 17, 158-163.

Kennelly, M. A., J. H. Dunlap, T. B. Sanford, E. L. Kunze, M. D. Prater and R. G. Drever, (1989a) The Gulf of Cadiz expedition: R/V Oceanus cruise 202. APLUW TR 8914, Applied Physics Laboratory, University of Washington, Seattle, WA, $115 \mathrm{pp}$.

Kennelly, M. A., T. B. Sanford, T. W. Lehman (1989b) CTD data from the Gulf of Cadiz Expedition: R/V Oceanus cruise 202. APL-UW TR 8917, Applied Physics Laboratory, University of Washington, Seattle, WA, 129 pp.

Kennelly, M. A., M. D. Prater, J. H. Dunlap. E. L. Kunze and T. B. Sanford, (1989c) XCP data from the Gulf of Cadiz Expedition: R/V Oceanus cruise 202. APLUW TR 8925, Applied Physics Laboratory, University of Washington, Seattle, WA, 206 pp.

Kenyon, N. H., and R. H. Belderson (1973) Bed forms of the Mediterranean undercurrent observed with side-scan sonar. Sedimentary Geology, 9, 77-99.

Killworth, P.D., (1977) Mixing on the Weddell Sea continental slope. Deep Sea Research, 24, 427-448.

Kinder, T.H., and H.L.Bryden, (1987) The 1985-1986 Gibraltar Experiment: data collection and preliminary results. EOS, 68, 786-787, 793-795.

Kinder, T. H., and G. Parrilla (1987) Yes, some of the Mediterranean outflow does come from great depth. Journal of Geophysical Research, 92, 2901-2906.

Knaus, J.A., (1969) A Note on the Transport of the Gulf Stream. Dup-Sen Res., 16.

Knauss, J. A. (1978) Introduction to Physical Oceanography. Prentice-Hall, Englewood CLiffs, New Jersey, 338 pp.

Lacombe, H. and C. Richez (1982) The regime of the Strait of Gibraltar. Hydrdynamics of semi-enclosed seas, J. C. J. Nihoul, editor, Elsevier Oceanography Series, Elsevier, Amsterdam, Vol. 34, 13-73. 
Lacombe, H. and P. Tchernia, (1960) Quelques Traits Generaux de l'Hydrologie Mediterranee. Cahiers Oceanographiques, 12, 527-547.

Lacombe, H., J. C. Gascard, J. Gonella and J. P. Bethoux, (1981) Response of the Mediterranean to the water and energy fluxes across its surface, on seasonal and interannuak scales. Oceanologica Acta, 14, 247-255.

Lascaratos, A., R. G. Williams and E. Tragou, (1993) A Mixed-Layer Study of the Formation of Levantine Intermediate Water. Journal of Geophysical Research, in press.

Lynch, J. and R. Lueck (1989) Expendable Dissipation Profiler (XDP) Data from the Mediterranean Out-Flow Experiment R/V Oceanus Cruise 202 Leg V. The Johns Hopkins University Chesapeake Bay Institute, Technical Report JHUCBI TR89-01, 284 pp.

Ludwick, J. C. (1975) Variations in the boundary-drag coefficient in the tidal entrance to Chesapeake Bay, Virginia. em Mar. Geol, 19, 19-28.

MacCready, P., and P. B. Rhines, (1991) Buoyant inhibition of Ekman transport on a slope and its effect on stratified spin-up. Journal of Fluid Mechanics, 223, 631-661.

MacCready, P., and P. B. Rhines, (1993) Slippery Bottom Boundary Layers on a Slope. Journal of Physical Oceanography, 23, 5-22.

Macdonald A. M., J. Candela and H. L. Bryden, (1993) Heat Transport in the Strait of Gibraltar. Accepted for publication in The Seasonal and Interannual Variability of the Western Mediterranean Sea, P. La Violette editor, Elsevier.

Madelain, F. (1967) Calculs dynamiques au large de la Pennisula Iberique. Cahiers Oceanographiques, 19, 181-194.

Madelain, F., (1970) Influence de la topographie du fond sur l'ecoulement Mediterranean entre le detroit de Gibralytar et le Cap Saint-Vincent. Cahiers Oceanographiques, 22, 44-61.

Maillard, C. (1986) Atlas Hyrologique de l'Atlantic Nord-Est. Institut Francais de cherche pour l'Exploitation de la Mer, Brest, 32 pp.

Malonotte-Rizzoli, P. and A. Hecht, (1988) Large-scale properties of the Eastern Mediterranean: a review. Oceanologica Acta, 11, 4, 323-335.

Marshall, J.C., A.J. Nurser, and R.G. Williams, (1993) Inferring the subduction rate and period over the North Atlantic. Journal of Physical Oceanography, submitted. 
McWilliams, J. C., (1988) Vortex generation through balanced adjustment. Journal of Physical Oceanography, 18, 1178-1192.

MEDOC GROUP, (1970) Observation of the formation of deep water in the Mediterranean Sea. Nature, 227, 1037-1040.

Miles, J. W., and L. N. Howard, (1964) Note on a heterogeneous shear flow. Journal of Fluid Mechanics, 20, 331-336.

Millard, R. C., W. B. Owens and N. P. Fofonoff (1990) On the calculation of the Brunt-Vaisala frequency. Deep Sea Research, 37, 167-181.

Millot, C., (1985) Some features of the Algerian current. Journal of Geophysical Research, 90, 7169-7176.

Millot, C., (1987) Circulation in the western Mediterranean sea. Oceanologica Acta, 10, 143-149.

Needler, G. T., and R. A. Heath, (1975) Diffusion coefficients calculated from the Mediterranean salinity anomally in the North Atlantic Ocean. Journal of Physical Oceanography, 5, 173-182.

Nielsen, J. N., (1912) Hydrography of the Mediterranean and Adjacent Waters. Report of the Danish Oceanographic Expedition 1908-1910 to the Mediterranean and Adjacent Waters, Copenhagen, 1, 72-191.

Nof, D., (1983) The translation of isolated cold eddies on a sloping bottom. Deep Sea Research, 30, 171-182.

Nof, D., (1990) The breakup of dense filaments. Journal of Physical Oceanography, 20, 880-889.

Nof, D., (1991) Lenses generated by intermittent currents. Deep Sea Research, 38, 1662-1672.

Ochoa, J., and N. A. Bray, (1991) Water mass exchange in the Gulf of Cadiz. Deep Sea Research, 38, Suppl. 1, 5465-5503.

Ovchinnikov, I. M., (1984) The formation of Intermediate Water in the Mediterranean. Oceanology, 24, 168-173.

Ozturgut, E., (1976) The Source and Spreading of the Levantine Intermediate Water in the Eastern Mediterranean. Saclant ASW Research Centre Memorandum SM-92, La Spezia, Italy, 45 pp. 
Pedersen, (1980) Dense bottom currents in rotating ocean. Journal Hydr-Asce., 106, pp. 1291.

Perkin, R. G., and E. L. Lewis, (1980) The practical salinity scale 1978: fitting the data. IEEE J. of Ocean. Eng., OE-5, 9-16.

POEM GROUP, (1992) General Circulation of the Eastern Mediterranean. Earth Science Review, 32, 285-308.

Pollard, O. M., P. B. Rhines, and R. O. R. Y. Thompson, (1973) The deepening of the wind-mixed layer. Geophysical Fluid Dynamics, 3, 381-404.

Prater, M. D., (1991) A method for depth and temperature correction of expendable probes. Journal of Atmospheric and Oceanic Technology, 8, 888-894.

Pratt, L.J., (1986) Hydraulic control of sill flow with bottom friction. Journal of Physical Oceanography, 16, 1970-1980.

Pratt, L. J., (1987) Rotating Shocks in a Separated Laboratory Channel Flow. Journal of Physical Oceanography, 17, 483-491.

Price, J. F., (1979) On the scaling of stress-driven entrainment experiments. Journal of Fluid Mechanics, 90, 509-529.

Price, J. F., R. A. Weller and R. Pinkel (1986) Diurnal Cycling: Observations and Models of the Upper Ocean Response to Diurnal Heating, Cooling, and Mixing. Journal of Geophysical Research91, 8411-8427.

Price, J. F. and M. O. Baringer (1993) Outflows. Journal of Physical Oceanography, submitted.

Price, J. F., M. O. Baringer, R. G. Lueck, G.C. Johnson, I. Ambar, G. Parilla, A. Cantos, M. A. Kennelly, and T. B. Sanford (1993) Mediterranean Outflow Mixing Dynamics. Science, 259, 1277-1282.

Reid, J. L., (1979) On the contribution of the Mediterranean Sea outflow to the Norwegian-Greenland Sea. Deep Sea Research, 26, 1199-1223.

Rhein, M. and H. H. Hinrichsen, (1993) Modification of Mediterranean Water in the Gulf of Cadiz, studied with hydrographic, nutrient and chlorofluoromethane data. Deep Sea Research, 40, 267-291.

Rhines, P., and P. MacCready (1989) Boundary Control over the Large-Scale Circulation, in Parameterizations of Small Scale Processes, Proceedings 'Aha Huliko's Hawaiian Winter Workshop, University of Hawaii at Manoa, P. Muller and D. Henderson, Ed.s, 75-98, 1989. 
Richardson, P. L., and K. Mooney, (1975) The Mediterranean outflow- a simple advective-diffusion model. Journal of Physical Oceanography, 5, 476-482.

Richardson, P. L., M. S. McCartney and C. Maillard, (1991) A search for meddies in historical data. Dynamics of Atmospheres and Oceans, 15, 241-265.

Schllitzer, R., W. Roether, H. Oster, H-G., Junghans, M. Hausman, H. Johannsen, and A. Michelato, (1991) Chloroflouromethane and Oxygen in the Eastern Mediterranean. Deep Sea Research, 38(12), 1531-1551.

Seidler G. (1968) Häufigkeitsverteil von Wasserarten im Ausstrombereich von Meeresstraßen. Kieler Meeresforchungen, 24, 59-65.

Smith, P. C., (1975) A streamtube model for bottom boundary currents in the ocean. Deep Sea Research, 22, 853-873.

Speer, K., and E. Tziperman, 1990. Convection from a source in an ocean basin. Deep Sea Research, 37, 431-446.

Stanton, B. R., (1983) Low frequency variability in the Mediterranean outflow west of Gibraltar. Deep Sea Research, 30, 7A, 743-761.

Sternberg, R. W. (1968) Friction factors in tidal channel with differing bed roughness. Mar. Geol., 6, 243-260.

Stommel, H. and A.B. Arons, (1960) On the abyssal circulation of the world ocean-I stationary planetary flow on a sphere. Deep Sea Research, 6, 140-154.

Stommel, H. and A.B. Arons, (1972) On the abyssal circulation of the world ocean-V The influence of bottom slope on the broadening of inertial boundary currents. Deep Sea Research, 19, 707-718.

Stommel, H., H.Bryden and P.Mangelsdorf, (1973) Does the Mediterranean outflow come from great depth? Pageoph, 105, 879-889.

Stommel, H. A., and H. G. Farmer, (1953) Control of salinity in an estuary by a transition. Journal of Marine Research, 12, 13-20.

Stommel, H. A. Voorhis and D. Webb, (1971) Submarine clouds in the ocean. American Scientist, 59, 716-722.

Sur, H. I., E. Ozsoy, T. Oguz and U. Unluata, (1992) Wide area formation of intermediate and deep water in the northern Levantine Basin. submitted to CIESM Congress, Trieste, Italy. 
Sverdrup, H. U., Johnson, M. W. and R. H. Fleming, (1942) The Oceans, Their Physics, Chemistry and General Biology. Prentice-Hall, Englewood Cliffs, N.J., $1087 \mathrm{pp}$.

Taylor, G. I., (1931) Internal waves and turbulence in a fluid of variable density. Rapp. P.-v. Reun. Cons. perm. int. Explor. Mer, 76, 35-43.

Thompson, J.D. and W.J. Schmitz, (1989) A limited-area model of the Gulf Stream: design, initial experiments and model-data intercomparison. Journal of Physical Oceanography, 19, 791-814.

Thompson, R. O. R. Y., (1973) Stratified Ekman boundary layer models. Journal of Fluid Mechanics, 5, 201-210.

Thorpe, S. A., (1971) Experiments in the instability of stratified shear flows: miscible fluids. Journal of Fluid Mechanics, 46, 299-319.

Trowbridge, J. H. (1992) Turbulent Mixing in Stress-Driven Stratified Flows. Journal of Fluid Mechanics, submitted.

Turner, J.S., (1973) Buoyancy effects in fluids. Cambridge University Press, Great Britian, 368 pp.

Turner, J. S. (1986) Turbulent entrainmnet: the development of the entrainment assumption, and its application to geophysical flows. Journal of Fluid Mechanics, $173,431-471$.

Warren, B. A., (1969) Diverenge of isobaths as a cause of current branching. Deep Sea Research, 16S, 339-355.

Whitehead, J. A., A. Leetmaa and R. A. Knox, (1974) Rotating hydraulics of strait and sill flow. Geophysical Fluid Dynamics, 6, 101-125.

Wimbush, M., and W. Munk, (1970) The benthic boundary layer. in The Sea, 4(1), A. E. Maxwell, ed., John Wiley and Sons, New York, 731-758.

Woods, J. D., and R. L. Wiley, (1972) Billow turbulence and ocean microstructure. Deep Sea Research, 19, 87-121.

Worthington, L.V., (1976) On the North Atlantic Circulation, The Johns Hopkins Oceanographic Studies, 6, 110 pp.

Wust, G., (1961) On the Vertical Circulation of the Mediterranean Sea. Journal of Geophysical Research, 66, 3261-3271. 
Zenk, W., (1975a) On the Mediterranean outflow west of Gibraltar. "Meteor" Forschungergebnisse, ser A, 16, 23-34.

Zenk, W., (1975b). On the origin of the intermediate double-maxima in T/S profiles from the North Atlantic. "Meteor" Forschungergebnisse, ser A, 16, 35-43.

Zenk, W. and L. Armi, (1990) The complex spreading pattern of Mediterranean water off the Portuguese continental shelf. Deep Sea Research, 36, 1805-1823. 